\title{
High-Speed Wavelength Swept Laser Source for Optical Coherence Tomography
}

\author{
Changho Chong \\ Department of Electrical and Computer Engineering \\ Graduate School of Engineering \\ Yokohama National University \\ Supervisor : Professor Yasuo Kokubun
}

March, 2009 

To my lovely wife, Ayumu, two daughters, Emma and Sally, and my son, Siwon. 

Everything should be made as simple as possible, but not one bit simpler

Albert Einstein 



\section{Abstract}

Optical Coherence Tomography (OCT) is one of promising technologies in medical diagnostic imaging system for the next generation, and which has been gaining great attentions these years. Swept-Source OCT (SS-OCT) that utilizes a tunable laser has advantages of higher sensitivity of $20 \mathrm{~dB}$ or greater, three times higher resolution, and faster imaging speed with several tens frame per second in comparison with conventional Time Domain OCT (TD-OCT). There are following correlations between image properties in SS-OCT and performance/parameters of swept source.

1) Coherence length of the source is proportional to the measurement depth range.

2) Wavelength swept range is counter-proportional to the axial resolution of the image.

3) Swept rate is proportional to the imaging speed because one sweep corresponds to an A-line (vertical line) of the OCT image.

So high-speed imaging of high-resolution, large depth OCT requires high-speed sweep, wide range, and high coherence (or narrower spectral linewidth) during sweep. However, most of previously introduced technologies have two significant drawbacks that make us difficult to implement it into actual medical equipment for real clinical application. One is the fact that these sources are not fully meeting the simultaneous requirement of three parameters. Particularly, coherence length degrades when the source is swept at very high speed over $20 \mathrm{kHz}$ or higher. In Ophthalmology or Cardiovascular applications, large depth range is required with $40-50 \mathrm{kHz}$, very high swept rate. Ambiguity removal process by having phase shifter device was proposed to countermeasure this issue, but it also complicates the system configuration. Secondly, the transient response of the sweep is intrinsically non-linear so that it necessitates very complex post-process to calculate the OCT signal, and which makes real-time imaging virtually impossible as a result.

The major purpose of this study is to propose and validate the novel configurations of swept source that overcome these drawbacks and improve the performance of swept source, thus improving the image quality of SS-OCCT.

Followings are major proposals and the work focused in this study;

1. Highly k-linear sweep for high-speed or real-time imaging

2. Coherence length improvement by Quasi-Phase Continuous Tuning

3. Wide range tuning for high resolution OCT imaging

4. Clinical applications 
First, a base configuration of a fiber extended cavity wavelength swept laser source was proposed which utilizes a polygon-scanner grating filter in Littrow arrangement that linearizes the frequency sweep. Geometrical compensation technique with using prism expander in the tunable filter was introduced. Theoretical analysis was carried out to estimate the linearity, and the prototype source was fabricated to validate its performance and to evaluate the OCT image quality. Linearity of less than $0.25 \%$ is achieved with extremely high repeatability between scans. And actual OCT image measured with the source proved that highly k-linear sweep eliminates the need of wavelength rescaling process and increase the imaging speed.

Second, we introduced the novel idea of Quasi-Phase Continuous Tuning. We have proven that without implementing mirror canceling technique or the other costly mitigation methods, coherence length can be drastically improved by using the proposed technique. Theoretical estimation of linewidth is also carried out.

Third, several schemes for wide range tuning with special filter geometries are proposed. These techniques overcome the physical limitation of tuning range with rather simple configurations.

In this thesis, these studies are divided in the chapters as in following. In Chapter 1, background of OCT and SS-OCT explains general discussion on the requirements for swept source in various specific applications. And it is followed by Chapter 2, 3, 4 describing the above-proposed innovations. SS-OCT algorithm and clinical study examples based on proposed swept source are introduced in Chapter 5 including novel MEMS fiber probe for endoscopic applications that was combined with SS-OCT system. In Chapter 6, feasibility studies on single mode hop-free swept lasers are discussed for the future improvement. 


\section{Table of Contents}

Chapter 1 Introduction ............................................................ 1

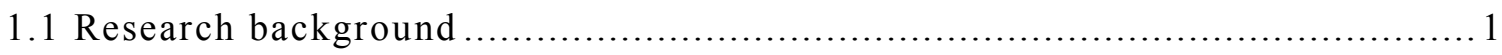

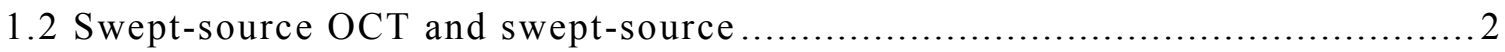

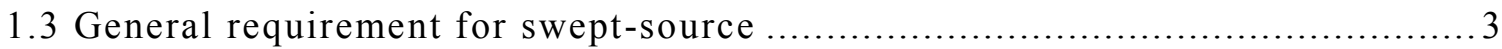

1.4 Requirements for specific OCT applications ................................... 6

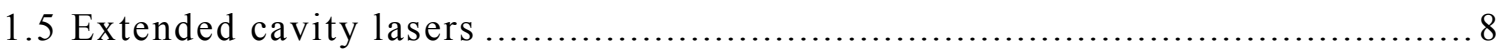

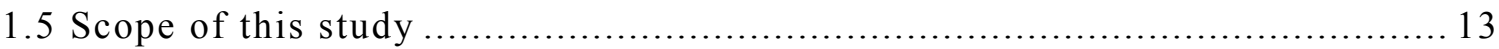

Chapter 2 General design of high-speed wavelength swept source and k-linear

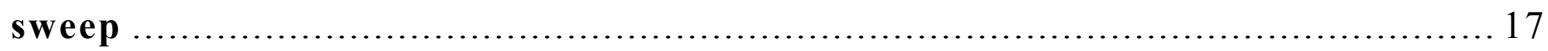

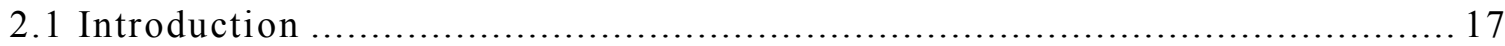

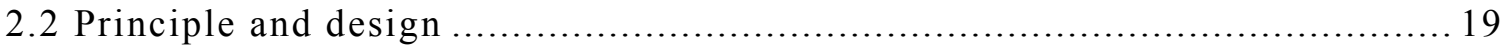

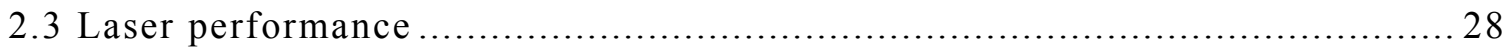

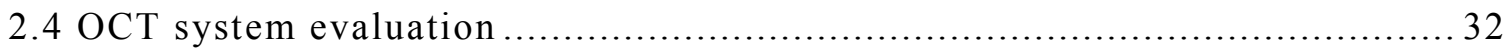

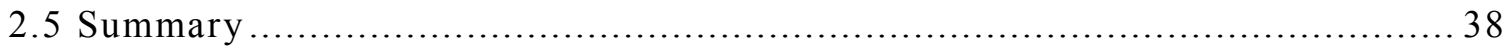

Chapter 3 Coherence length improvement by quasi-phase continuous tuning

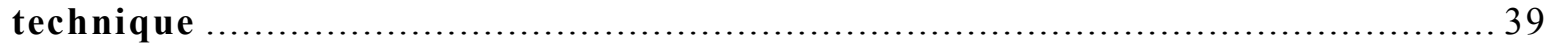

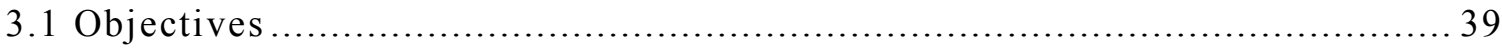

3.2 Concept of Quasi-Phase Continuous Tuning ..................................... 41

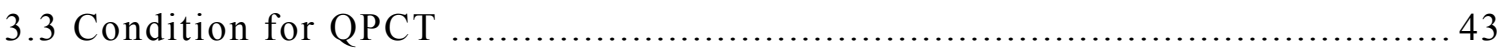

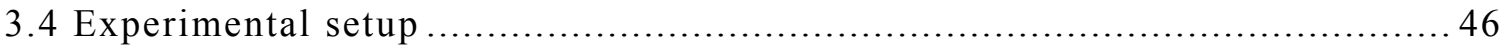




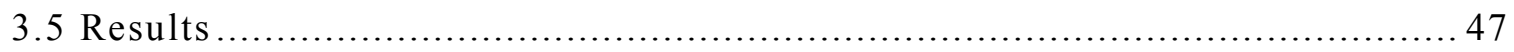

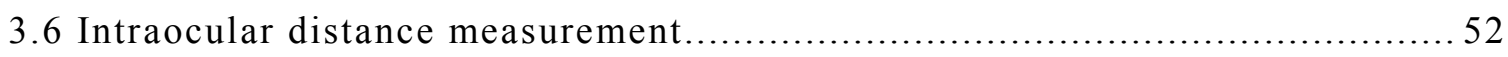

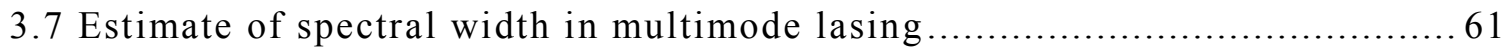

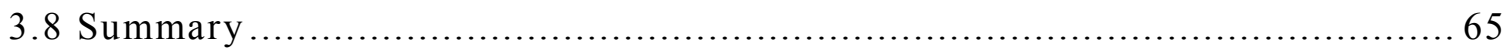

Chapter 4 Wide range sweep for high-resolution SS-OCT .......................... 67

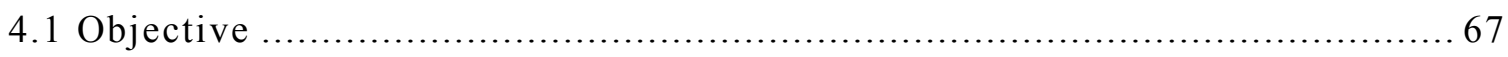

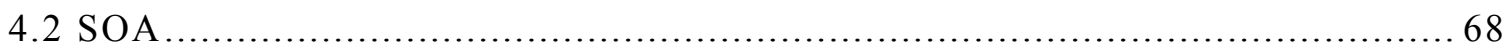

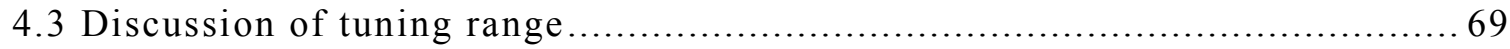

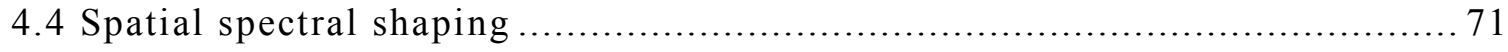

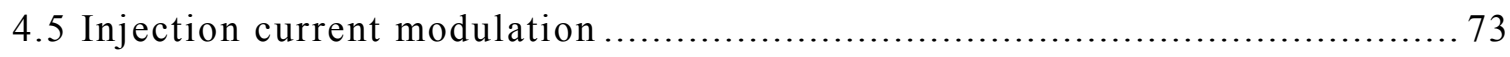

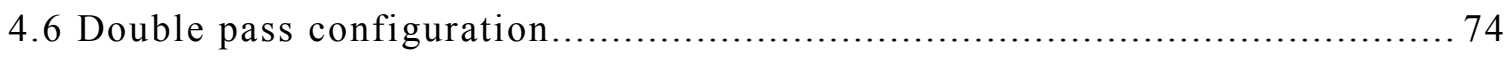

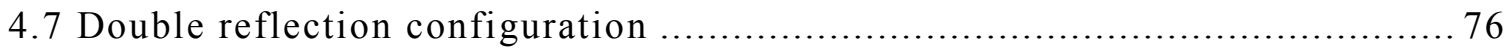

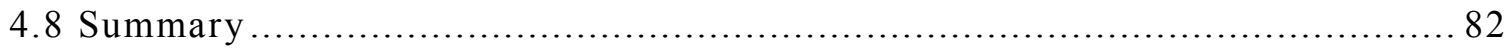

Chapter 5 SS-OCT system demonstration and clinical studies ....................... 83

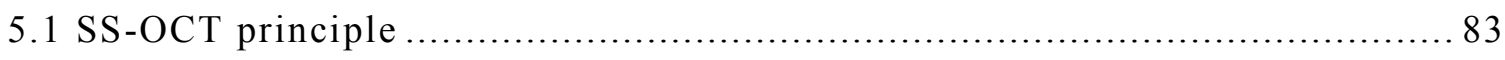

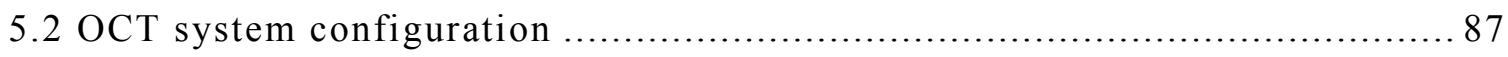

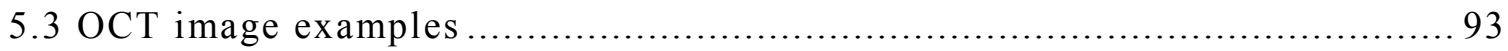

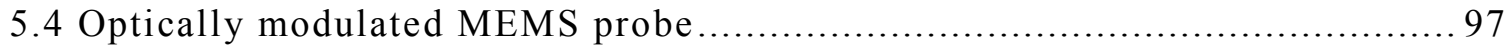

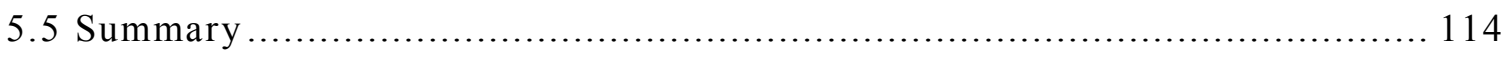

Chapter 6 Feasibility study on single-mode continuous sweep..................... 115

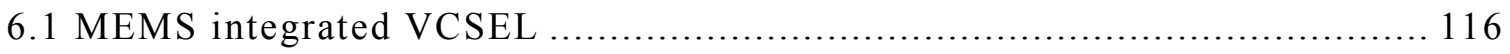

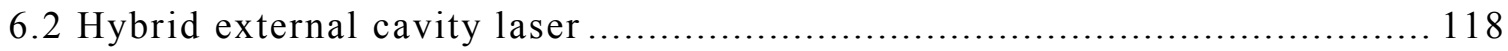


6.3 Fiber ECL with saturable absorber ........................................... 121

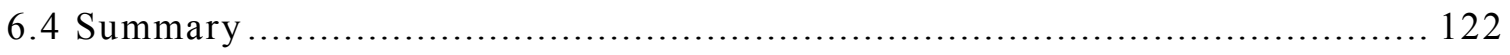

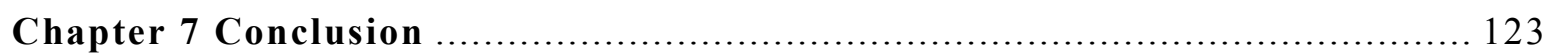

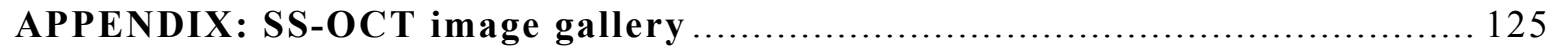

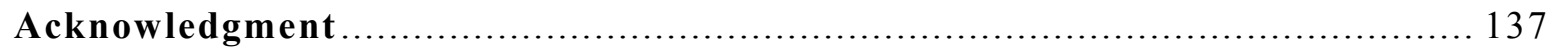

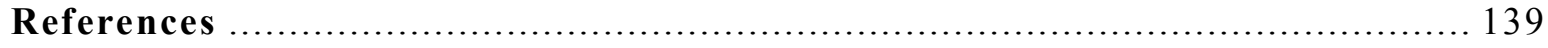

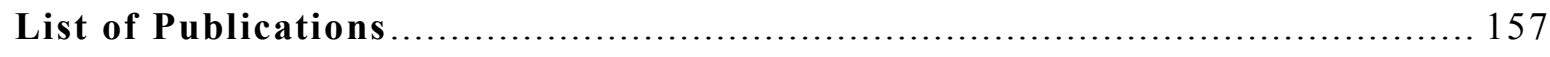

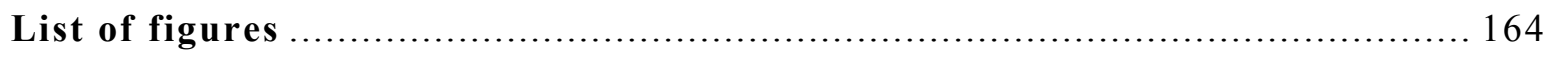

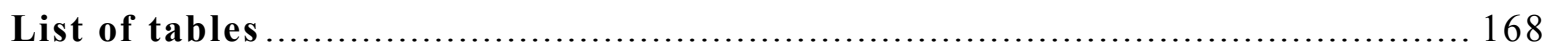




\section{Chapter 1 Introduction}

\subsection{Research background}

Continuous and repetitively tunable or "swept" lasers have become common in both research laboratories and production environments for measuring optical parameters. The lasers have a narrow spectral line width that is swept rapidly over a wide wavelength range. The light is used as a "probe" for measuring spectral response, for example absorption, light scattering or to measure surface topography using the interference effects of the coherent light. Compared to systems using broadband light sources with optical spectrum analyzers these lasers provide high sensitivity and a rapid measurement time; but nevertheless are still limited by a scan time of typically one second or more for a $100 \mathrm{~nm}$ scan.

This study proposes a high speed-scanning laser that continuously scans over a $110 \mathrm{~nm}$ wavelength range at up to $20 \mathrm{kHz}$. This scan speed, which is some 20,000 times faster than conventional swept lasers, opens up a wealth of new application areas some of which have only just started to be researched. Spectroscopic measurements that have been performed with conventional swept lasers can now be performed in real time and, further, transient dynamics can also be monitored. This is critical for applications such as gas analysis in internal combustion engines or industrial processes [1-5], or monitoring vibrations with fiber Bragg gratings in fiber optic sensor systems [6]. The most significant application, however, is real time imaging using a technique called Optical Coherence Tomography (OCT) [7-12]. For this application the rapid scan is indispensable and the technology is actively being researched for medical applications in particular [13-15].

In this section, we describe first, the research background of Swept-Source OCT followed by general requirements as well as application-specific requirements of the swept source. We, then, explain what technology is best fit to achieve the required performance while describing the drawbacks of previous works. Finally, we explain the scope of this thesis and contents of this study. 


\subsection{SS-OCT and swept source}

OCT is non-invasive imaging technique that was originally introduced in early 90 's, and diagnosis systems based on which are now widely practiced in ophthalmology application. Compared to conventional medical imaging technologies such as Magnetic Resonance Imaging (MRI), Positron Emission Tomography (PET), X-Ray Computerized Tomography (CT) and Ultrasonography, OCT provides a safe, high resolution solution at a cost point that will enable widespread use in hospitals and clinics. Most of these OCT systems use Time-Domain optical interferometry in which the optical path length difference between reference mirror and sample in the Michelson or Mach-Zehnder interferometer is modulated in time. Time-Domain or TD-OCT had opened up the potential of optical biopsy but there is performance limitation for further extension of the applications. First, imaging speed is relatively slow because of mechanical delay modulation. Second, even when higher frequency scanning is possible, detection sensitivity drops because of detection bandwidth in return. Fourier-Domain OCT $[16,17]$ is a break-through technology to achieve high sensitivity and high speed imaging at the same time. FD-OCT relies on analyzing the individual frequency components of backscattered light from the sample or tissue. There are two ways within FD-OCT. One is Spectral-Domain OCT (SD-OCT) that uses low coherence light source and spectrometer where frequency components are spatially analyzed on the CCD array. Fast readout speed of $\mathrm{CCD}$ benefits to the imaging speed and high signal-to-noise ratio (SNR) gives 20-30dB advantage over conventional TD-OCT [18,19]. However, there are also disadvantages. Image gets blurred and degraded when the sample arm motion washes out interference fringe on the CCD during pixel integration time [20,21]. Unavailability of InGaAs CCD with higher pixel resolution also limits the application of FD-OCT in in-vivo endoscopic applications.

The other approach is Swept-Source OCT that uses continuous and repetitively tunable or "swept" light source where frequency components are analyzed in time with a single photodetector. Each wavelength scan generates depth information; lateral scanning of the laser beam then enables a cross section image to be constructed. This technique has a theoretical sensitivity benefit equal to that of SD-OCT, while overcoming the disadvantage of SD-OCT such as fringe washout and allowing the use of longer wavelength over $1 \mu \mathrm{m}$ to $1.5 \mu \mathrm{m}$ range for endoscopy.

Here the question is how we can realize the "swept-source" that meets requirements for the use in OCT while meeting the same cost-performance as "CCD".

Next section explains correlation of source performance to that of SS-OCT and other general requirements for the source. 


\subsection{General requirements for the swept source}

\section{Output power}

The higher the output power on the sample, the higher the SNR improves in principle [23]. However, maximum permissible exposure on the human tissue is regulated by ANSI(American National Standards Institute) standard. $0.7 \mathrm{~mW}$ in average at $800 \mathrm{~nm}$ range and about $1 \mathrm{~mW}$ at $1060 \mathrm{~nm}$ range at the probe arm are allowable exposure for ophthalmic applications. The other tissues can be exposed over $>10 \mathrm{~mW}$ in endoscopic applications. The use of further longer wavelength such as $1300 \mathrm{~nm}$ makes the max power less critical in terms of ionization of tissue by high power exposure.

\section{Sweep linearity}

OCT signal is processed by time-sampling the backscattered light as the swept source sweep the wavelength followed by Fourier transform (FFT). Ideally, the sweep should be linear in $k$ space $(k=2 \pi / \lambda)$. But actual sweep curve of the most of proposed swept sources are non-linear in time because of its intrinsic tuning mechanism. For examples, the use of Galvano mirror [24], or fiber Fabry-Perot filter imposes sinusoidal sweep due to its driving characteristics [25]. If simply applying FFT on time-sampled interferogram when this non-linearity is present, it blurs resolution of the signal and signal power also decays. So, in general, most of SS-OCT systems implement either nonlinear sampling with the use of optical clock having another set of interferometer and detector, or post processing approach; so called "wavelength rescaling process".

\section{Wavelength range}

The choice of wavelength band in OCT is dependent on the water absorption and scattering property of the sample or tissue of interest [26-29]. Figure 1.1 shows the absorption and scattering coefficient over visible to infrared wavelength bands. In general, $800 \mathrm{~nm}$ range is used for retinal imaging because of low absorption in vitreous humor [30,31], and recently $1060 \mathrm{~nm}$ range gains attentions because of large penetration in retinal tissue as well as low dispersion property in tissue[32-34]. In endoscopic application $1310 \mathrm{~nm}$ range or longer range is commonly used because of low scattering resulting in large depth penetration [36,37]. The OCT in $1310 \mathrm{~nm}$ range has another benefit; abundant available optical components in this range that were developed for optical fiber telecommunication applications. Spectral width of OCT signal, i.e. axial resolution is inversely related to the envelope of temporal power profile via Fourier transforms, and which is given by equation; 


$$
\delta z=\frac{2 \ln 2}{\pi} \frac{\lambda_{0}^{2}}{n \Delta \lambda}
$$

For examples, $10 \mathrm{um}$ resolution can be achieved by $30 \mathrm{~nm}$ at $820 \mathrm{~nm}$ and $75 \mathrm{~nm}$ at $1310 \mathrm{~nm}$, respectively.

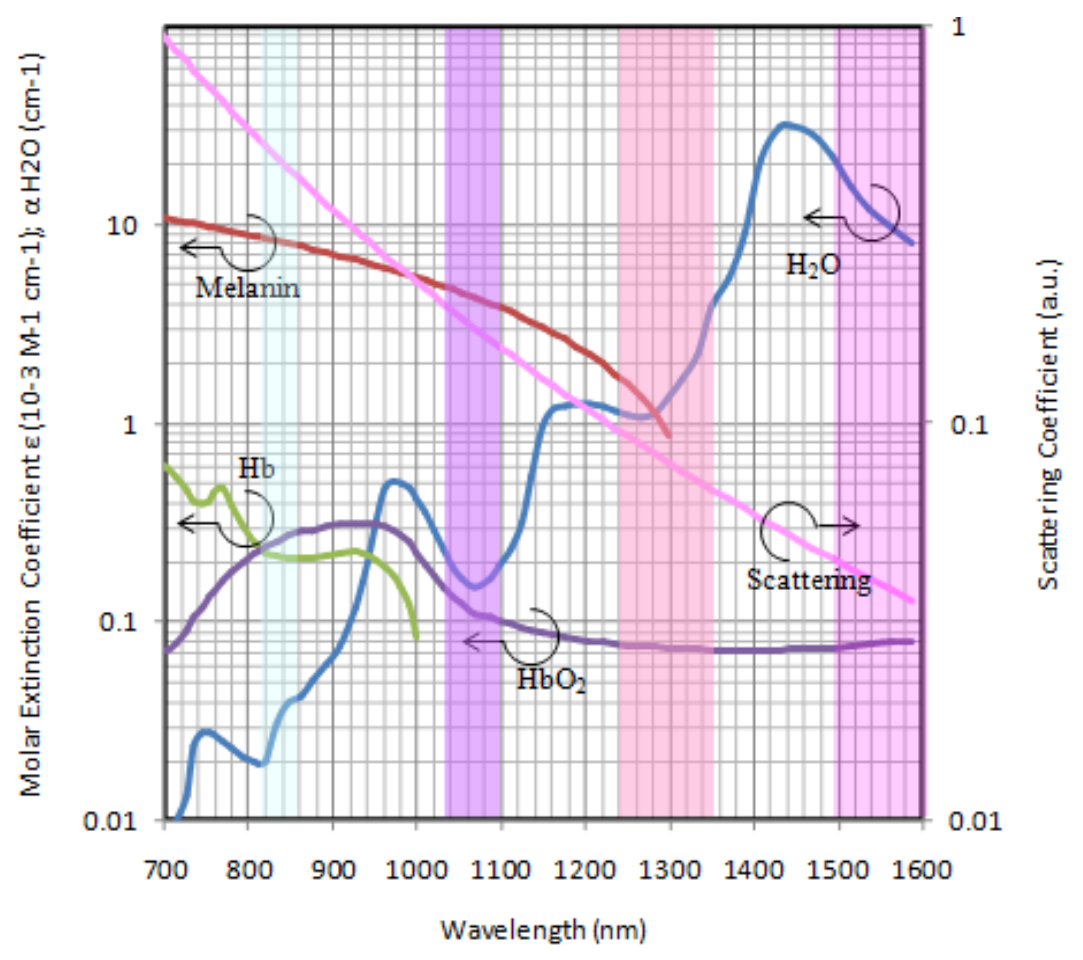

Figure 1.1 Absorption and scattering coefficients of light

Swept rate (scanning speed)

Wavelength swept rate, or scanning speed of the swept source is directly reflected on the imaging speed like readout speed or refresh rate of CCD in SD-OCT. Swept rate corresponds to A-line rate in OCT. Increasing A-line rate makes it possible to accommodate more A-lines per frame or increase the frame rate. In practical applications the ability to produce video rate images is of critical importance. This not only removes imaging artifacts that is creased by undesired movement, but also enables a large area/volume measurement without compromising resolution in a short amount of time. Depending on applications swept rate of $10 \mathrm{kHz}$ to $100 \mathrm{kHz}$ range is required.

\section{Instantaneous linewidth / Coherence length}

Instantaneous linewidth; this terminology is very specific to the high-speed swept-source. Sometimes it is called, dynamic linewidth. It doesn't mean linewidth of a single longitudinal 
mode as usually referred in the laser oscillation. The most of swept sources for SS-OCT are partially coherent light sources, and spectral width is the width of envelope that contains group of cavity modes. Since instantaneous linewidth is finite and the OCT signal is convolution of its spectrum and interferogram, its fringe visibility drops for the higher frequency components, i.e. at deeper range. Coherence length is defined as the optical round trip delay or twice of depth range where fringe visibility drops half or Fourier-transformed OCT signal drops $6 \mathrm{~dB}$ compared to the signal power at zero delay. The relation of instantaneous linewidth, $\delta \lambda$ and coherence length, $C_{l}$ is given in equation;

$$
C_{l}=\frac{2 \ln 2}{\pi} \frac{\lambda_{0}{ }^{2}}{\delta \lambda}
$$

Coherence length of most of medical applications ranges between 2 and $10 \mathrm{~mm}$ except for intraocular length measurement as discussed in Chapter 5.

\section{Relative Intensity Noise}

Intensity noise of the source at the signal detection band directly impacts on the SNR of the OCT system. Source of the noise attributes to the injection current from the driver circuitry and the cavity mode beating and its harmonics at the frequency equal to the reciprocal of cavity life time. Ripple on the spectral profile of the sweep also influences on the OCT signal as an alias noise. In OCT system the source RIN can be reduced if balanced detection is used for common mode noise reduction. RIN can be measured with a photodiode and an electrical spectrum analyzer.

\section{Polarization}

Single polarization output or output with high degree of polarization (DOP) is desired to suppress the effect of PMD in the interferometry system. Single polarization output may be useful when polarization sensitive OCT can be realized by adapting the Muller matrix method.

\section{Reliability}

Long term reliability and durability of the source is desired for harsh environmental conditions in clinical use. Meeting EMC/EMI standards is also important so that the instrument doesn't interfere with the other surrounding equipments. In most cases, 5 years product life should apply, corresponding to over average 40,000hrs of continuous usage. 


\subsection{Requirements for specific OCT applications}

The above explained three parameters; Swept rate and wavelength range and coherence length are interrelated and have been selected to provide optimum imaging resolution, imaging depth and imaging speed for OCT. These parameters can be reconfigured for different imaging requirements or applications.

Figure 1.2 shows typical examples of requirements for different applications. Here, swept range and instantaneous linewidth are enclosed as a parameter of Finesse; the ratio of instantaneous linewidth to swept range.

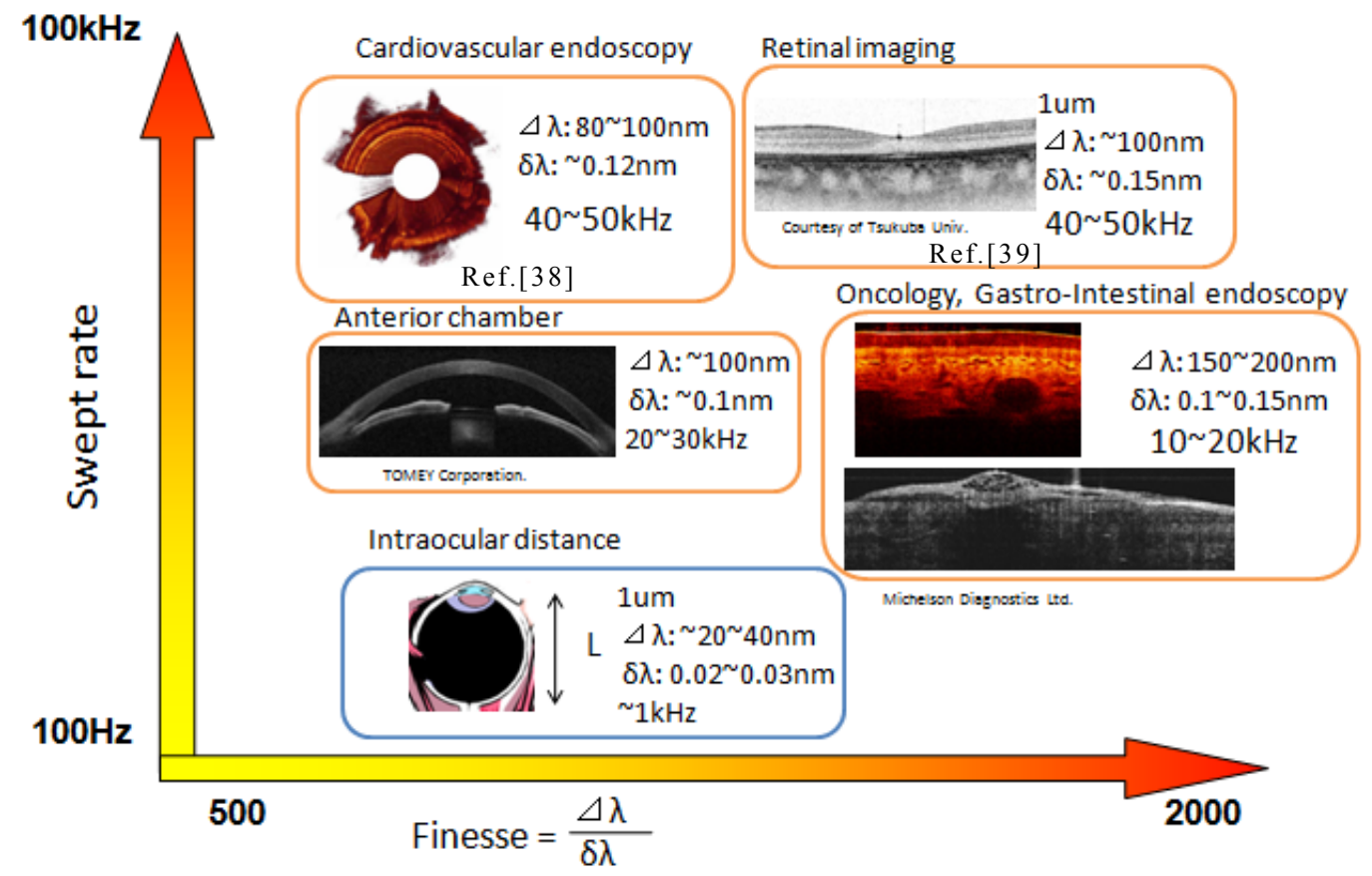

Figure 1.2 Requirements of swept source for different clinical application

Cardiovascular applications: Three dimensional structure and properties of plaque formed inside the coronary is the interest of imaging. Saline flush process to image the sidewall of the vessel allows only several seconds for the image acquisition of a few centimeter long volume with push-pull method [40]. A-line rate required here is 40 to $50 \mathrm{kHz}$. Since this is not a molecular level biopsy like cancer detection, axial resolution of $10 \mathrm{um}$ is sufficient for most of diagnosis that corresponds to about 80 to $100 \mathrm{~nm}$ swept range in $1310 \mathrm{~nm}$ wavelength range.

Gastro-Intestinal endoscopic applications: Cancer detection is everybody's ultimate goal as optical biopsy. In GI-endoscopy, axial resolution is the most critical parameter in this regard. 
Requirement of swept rate is somewhat compromised in this case even though 10 to $20 \mathrm{kHz}$ is necessary to mitigate the motion of sample and endoscope's probe.

Ophthalmic applications:

(1)Retinal imaging: Retinal tissue consists of multiple thin layers. High resolution is important factor here again. Eye ball is constantly vibrating at the frequency of several Hz. So, major glaucoma assessment requires whole three-dimensional tomograph to be captured within a few seconds in order to register the mapping of different nerve layers. Because thickness of retina to the bottom (Sclera) is relatively small in the order of $1 \mathrm{~mm}$, coherence length can be smaller in this application. $840 \mathrm{~nm}$ or $1060 \mathrm{~nm}$ band is chosen for the wavelength band because of low absorption in vitreous humor.

\section{(2) Anterior chamber}

A diversity of diagnosis in this area includes early diagnosis of glaucoma, post-LAISK, and iris tumor. Shape analysis is important aspect of anterior segment pathologies. So the depth range needs to reach down to the lens surface that is about $7 \mathrm{~mm}$ in physical depth. However, since cornea and vitreous gel is transparent and less scattering compared to non-ophthalmic human tissues, ex. skin, the decay of OCT signal due to finite coherence length does not fade the image contrast as abruptly as it does for skin. Hence, $5 \mathrm{~mm}$ coherence length is sufficient for most of cases. The use of $1.3 \mu \mathrm{m}$ range is advantageous in terms of getting clear contour in less scattering surfaces and deeper penetration near iridocorneal angle opening [41].

\section{(3)Intraocular distance measurement:}

Axial length of eye, or so-called intraocular distance [15] is the important parameter that needs to be accurately measured prior to intraocular lens implantation. The axial length ranges 20 to $35 \mathrm{~mm}$ for the normal human eye. Since this is only one-dimensional length measurement, swept rate can be around $1 \mathrm{kHz}$ or higher. In order for the light to reach retina, $1060 \mathrm{~nm}$ range or lower wavelength range should be chosen to minimize the absorption of water in vitreous humor.

\section{Hard and soft oral tissues or dental applications:}

Oral cancer and caries detection are interest of diagnosis for OCT [42]. The requirement is similar to that for GI-endoscopy, except for dental hard tissues, i.e. tooth. Since a tooth is made of enamel and dentin that have less water presence, less than $10 \%$ of volume, $1.3 \mu \mathrm{m}$ and $1.55 \mu \mathrm{m}$ ranges are useful especially for caries detection. 


\subsection{Extended cavity lasers}

Tunable lasers

So what type of tunable laser meets these requirements? In tunable lasers, there are many variations of technology. But no particular technologies other than extended cavity

Table 1.1 Comparison of different tunable laser technologies

\begin{tabular}{|c|c|c|c|c|c|c|}
\hline \multirow{2}{*}{ Type of tunable lasers } & $\begin{array}{c}\text { Tuning } \\
\text { mechanism }\end{array}$ & $\begin{array}{c}\text { Tuning } \\
\text { range } \\
{[\mathrm{nm}]}\end{array}$ & $\begin{array}{c}\text { Continuo } \\
\text { us tuning }\end{array}$ & Speed & Comments & Ref \\
\hline \multirow{2}{*}{ Grating } & 200 & 0 & Slow & Pivot tuning & \\
\cline { 2 - 8 } & AO filter & 160 & $\times$ & Fast & & 43 \\
\cline { 2 - 8 } & $\begin{array}{c}\text { Ring } \\
\text { resonator }\end{array}$ & 132 & $\times$ & - & Vernier effect & 44 \\
\hline & SFB array & 47 & $\triangle$ & Fast & $\begin{array}{c}\text { Need a } \\
\text { booster SOA }\end{array}$ & 45 \\
\hline
\end{tabular}

lasers suffice the requirements of swept source for wide range tunability in the first place. (see table 1.1). For a monolithic device like DFB array, tuning range per device is limited by principle and cascading in arrays necessitates the need of a booster amplifier. SSG DBR, on the other hand, can tune over $100 \mathrm{~nm}$ but its discrete tuning nature requires a quite complex control of multiple nodes that leads to rather slower tuning despite the fast response time of device itself. Tunable vertical cavity surface emitting laser (VCSEL) is the only one approach that can realize true continuous single mode tuning but output power and tuning range is limited to a few milliwatt and several tens of nanometers, respectively at this moment.

\section{Extended cavity lasers}

Extended cavity lasers or external cavity lasers (ECL) are common sources for transmitter for the long-haul transmission in telecommunication as well as tunable sources for measurement $[43,44]$. The most of these lasers operate in a single longitudinal mode oscillation. It is difficult to achieve wide range and high-speed sweep operation simultaneously in these lasers because mode-hop free operation during the rapid sweep is 
complex, and the control of which is time-limited process.

On the other hand, most of high-speed swept source lasers for OCT that have been introduced since early 2000's have ECL structure with a relatively long cavity [47-49]. The cavity is typically extended meters long with using a single mode fiber so that oscillation occurs with multiple of cavity modes within the Gaussian envelope of the tunable filter, and which can be gradually shifted like a tidal wave on the cavity modes as shown in Fig.1.3. Hence, the continuity of tuning, or "sweep" is ensured by this mode of operation. However, in strictly saying, this mode of operation is no longer "high-coherence" laser, it should rather called, "partially coherent" or "coherent amplification of ASE". This fact imposes us to compromise the coherence length, but achieving several millimeters is in fact sufficient for the most of OCT application. In this thesis, we also adapt the long extended cavity structure for realizing a swept-source. In the following discussion, we explain three key elements/devices suitable for configuring a swept source for OCT applications.

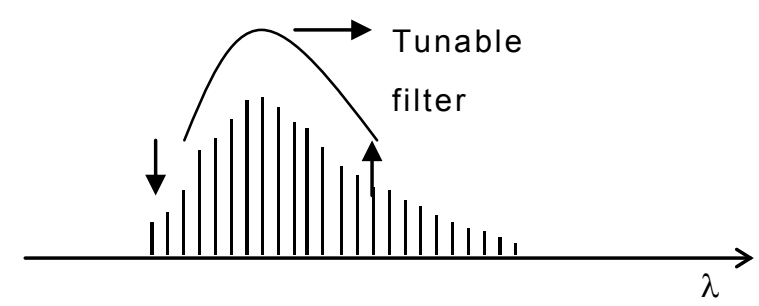

Figure 1.3 Spectral dynamics of a swept-source

\section{Semiconductor Optical Amplifier (SOA) as gain medium}

Semiconductor Optical Amplifier (SOA) is considered most suitable for a gain medium of swept source. It is sometimes just called "gain chip" when it is integrated into the device. SOA offers a wide range of selection of wavelength bands, ranging from 0.8 um to 1.6 um with use of either GaAs or InP based III-V compound semiconductors. Gain bandwidth can be typically over $100 \mathrm{~nm}$ at $1.3 \mathrm{um}$ range that is optimized by carefully engineering the bandgap structure of quantum well. Response time of SOA as short as sub nanoseconds is the most of interest in the use for swept source because over $\mathrm{kHz}$ rapid sweep requires intensity modulation in the order of nanoseconds on the longitudinal mode. Gain medium such as rare earth doped fiber amplifier has similar performance in gain level and bandwidth but response time of milliseconds is too slow for rapid sweep greater than $\mathrm{kHz}$ swept rate.

\section{Grating based filter}

Grating based filter either in Littrow or Littman-Metcalf configuration is considered best-fit as a tunable filter for the swept-source. Several reasons for this; one is filter shape. 
Filter shape is in Gaussian profile with box-like shape while Fabry-Perot filter has very peaky and not enough suppression over FSR(Free Spectral Range), and also monotonically decreasing at its slope while AO(accousto-optic) filter has undesired side-lobes that lead to insufficient suppression of ASE during lasing operation. FSR of grating filter is essentially large with appropriate geometry and grating constant. The other reason is that it can be designed into a single polarization selective filter so that oscillation of swept source can be locked in a single polarization within a cavity. Fluctuation of polarization because of undetermined polarization state with polarization insensitive devices leads to polarization phase noise during swept operation that affects on imaging quality as a result.

Wavelength tuning can be realized by changing the incident angle on the grating and which can be repetitively changed by beam deflector if using a resonant mirror or a polygon scanner.

\section{Polygon scanner}

Polygon scanner is industrially matured beam deflection device that is used for a broad range of applications. Motor can rotate up to the speed of 50,000 revolutions per minute when using air-bearing axis. Repeatability of beam deflection by using the polygon mirror is quite high; timing jitter less than $0.1 \%$, constancy of speed less than $0.025 \%$, facet-to-facet angle variation less than $0.2 \mathrm{mrad}$, which can realize highly constant and repeatable wavelength sweep over time. High repeatability also enables us to take advantage of wavelength rescaling process in a post-processing method with a pre-calibrated table in the case that non-linearity of sweep should be compensated. However, there is a design rule that restrains FSR of the tunable filter and swept rate. This is discussed in Chapter 4. 


\section{Previous works}

There are two major approaches that have been studied in MIT and Harvard, and frequently cited by many other works in SS-OCT area. The most of them are all based on extended cavity laser structure as previously mentioned.
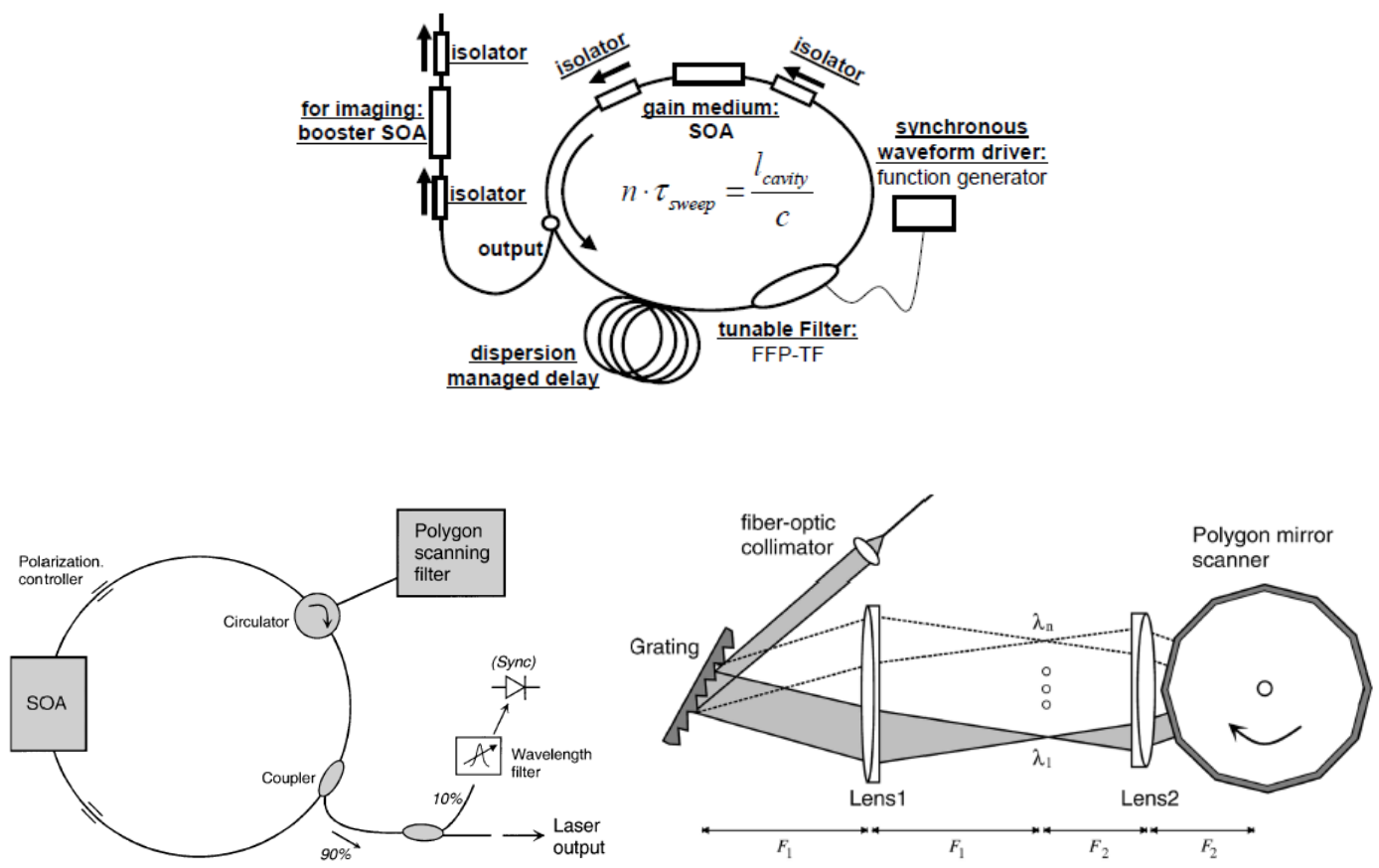

Figure 1.4 Fourier Domain Mode Locked laser source (top), ECL with polygon scanner-based filter with Telescopic lens configuration (figures taken from ref. [50], and [51] respectively).

One is Fourier Domain mode locked laser (FDML) [50]. In this laser, a dispersion managed large delay with long rounds of fiber is incorporated into the laser cavity and the narrowband optical filter is tuned periodically at the cavity round-trip time, or a harmonic of the round-trip time (Fig. 1.4 (top)). This produces a quasi-stationary mode of operation. Light from one frequency sweep propagates through the cavity and returns to the filter at the exact time when the transmission window of the optical bandpass filter is tuned to the same optical frequency. This laser has advantage of high-swept rate over $100 \mathrm{kHz}$ maintaining several millimeter coherence length. However, its sinusoidal sweep necessitates the need of real time wavelength rescaling process. And manufacturability is still questionable because of long rounds of fiber that needs to be precisely controlled in length with centimeter order and also polarization should be carefully managed over time.

The other notable approach is similar to the configuration proposed in this thesis; polygon scanner based filter with telescopic lens system [51]. Geometry is in Littman configuration but beam deflection is converted to converging angle via telescopic lens system so that the beam is effectively scanned for wavelength tuning. This configuration has limitation in size 
and Finesse. Bulky configuration is also problematic when ensuring durability of the source and its long-term reliability.

Table 1.2 shows performance of a variation of swept-sources proposed from leading research groups including MIT and Harvard.

Table 1.2 List of references of swept sources

\begin{tabular}{|c|c|c|c|c|c|c|}
\hline Date & Author & Institution & Technology & $\begin{array}{l}\text { Main Characteristics } \\
\text { Swept rate/range/ coherence length }\end{array}$ & Finesse & Ref \\
\hline $\begin{array}{l}\text { October } \\
2003\end{array}$ & SH.Yun & Harvard(MGH) & $\begin{array}{l}\text { Telescopic lens } \\
\text { +Polygon scan }\end{array}$ & $15.7 \mathrm{kHz} / 73 \mathrm{~nm} / 6 \mathrm{~mm}(0.127 \mathrm{~nm})$ & 575 & 51 \\
\hline $\begin{array}{l}\text { MARCH } \\
2005\end{array}$ & WY. On & Harvard(MGH) & $\begin{array}{l}2 x(\text { Telescopiclens } \\
+ \text { Polygon scan })\end{array}$ & $20 \mathrm{kHz} / 145 \mathrm{~nm} / 3 \mathrm{~mm}(0.25 \mathrm{~nm})$ & 580 & 52 \\
\hline $\begin{array}{l}\text { December } \\
2005\end{array}$ & $\begin{array}{l}\text { R.Huber JY. } \\
\text { Jiang }\end{array}$ & $\begin{array}{l}\text { MIT } \\
\text { Thorlabs }\end{array}$ & $\begin{array}{l}\text { Galvano, Free-space } \\
\text { cavity }\end{array}$ & $16 \mathrm{kHz} / 133 \mathrm{~nm} / 4 \mathrm{~mm}(0.19 \mathrm{~nm})$ & 700 & 53 \\
\hline $\begin{array}{l}\text { December } \\
2005\end{array}$ & SH.Yun & Harvard(MGH) & $\begin{array}{l}\text { Dual telescopic } \\
\text { lens+polygon }\end{array}$ & $115 \mathrm{kHz} / 80 \mathrm{~nm} / 3.3 \mathrm{~mm}(0.23 \mathrm{~nm})$ & 348 & 54 \\
\hline April 2006 & R.Huber & MIT & FDML & $59 \mathrm{kHz} / 74 \mathrm{~nm}(\mathrm{FWHM}) / 12 \mathrm{~mm}(0.063 \mathrm{~nm})$ & 1670 & 50 \\
\hline $\begin{array}{l}\text { August } \\
2008\end{array}$ & $\begin{array}{l}\text { SMR.Motag } \\
\text { hian Nezam }\end{array}$ & Caltec & $\begin{array}{l}\text { Dual reflection with } \\
\text { Littman arrangement }\end{array}$ & $50 \mathrm{kHz} / 91.6 \mathrm{~nm} / 6 \mathrm{~mm}(0.12 \mathrm{~nm})$ & 760 & 55 \\
\hline
\end{tabular}

Although tunable laser technologies have been studied over $30 y e a r s$, there had been no introduction of practically usable source meeting three important parameters since the concept of SS-OCT was first introduce by Fercher (Wien University) in 1995 [15]. Since around 2004, several proposals of swept sources have been reported. However, most of these have two significant drawbacks that make us difficult to implement it into actual medical equipment for real clinical application. One is the fact that these sources are not fully meeting the simultaneous requirement of three parameters. Particularly, coherence length degrades when the source is swept at very high speed over $20 \mathrm{kHz}$ or higher. In Ophthalmology or Cardiovascular applications, large depth range is required with $40-50 \mathrm{kHz}$ very high swept rate. Ambiguity removal process by having phase shifter device was proposed to countermeasure this issue [56-59], but it also complicates the system configuration. Secondly, the transient response of the sweep is intrinsically non-linear so that it necessitates very complex post-process to calculate the OCT signal, and which makes real-time imaging impossible as a result. 


\subsection{Scope of this study}

$1.3 \mu \mathrm{m}$ and $1.06 \mu \mathrm{m}$ range swept sources are very attractive light sources for SS-OCT in many different clinical applications. Adaptation of SS-OCT in actual diagnostic system has just started.

The major objective of this study is to propose the high-speed wavelength swept source that achieves simultaneous realization of high-speed, high-coherence (narrow linedwidth), wideband sweep. Experimental and theoretical study was also carried out for several specific applications.

What we are targeting in terms of specifications in comparison with previous works is better balanced laser. If the swept source design allows sufficient design margin, it means that it is capable of either achieving equally superior on three key parameters (i.e. swept rate, swept range, coherence length), or emphasizing extremely high performance on one specific parameter for a specific OCT application. Target swept rate ranges from 2 to $50 \mathrm{kHz}$ and swept range up to $160 \mathrm{~nm}$ without compromising on coherence length with any cases.

We propose and demonstrate three major technical innovations in this thesis.

1. Highly k-linear sweep for high-speed or real-time imaging

Accurate information about the wavelength throughout the scan is critical in generating an image with accurate proportions. Wavelength meters or monitoring devices are too slow in response to monitor the instantaneous wavelength at any point during a scan. We have addressed this issue optically with compensating optics inside the tunable filter. Linearity, defined as the frequency deviation/error from a linear extrapolation in time, of less than $0.2 \%$ is achieved with extremely good repeatability between scans.

2. Coherence length improvement by Quasi-Phase Continuous Tuning

Deeper penetration is always desired for most of OCT applications. We have proven that without implementing mirror canceling technique or the other costly mitigation method, coherence length can be improved by the proposed technique.

3. Wide range tuning for high resolution OCT imaging

Wide range tuning techniques and special filter geometries based on polygon scanner are proposed. These techniques overcome the physical limitation of tuning range with rather simple configuration.

In this thesis, Chapter 1 is devoted to introductory for the background of OCT and SS-OCT discussing general requirements for the swept source. Review of previous works is introduced to highlight the important parameters that should be overcome. 
Chapter 2 describes basic configuration of the proposed swept source, followed by the design for highly k-linear scan/sweep. Other basic performance of the source is also descried. OCT image quality with and without wavelength rescaling process is compared for the validation of the fabricated k-linear source.

In Chapter 3, coherence length improvement is demonstrated by Quasi-Phase Continuous Tuning (QPCT) technique. Concept of this technique is discussed and performance in $1.3 \mathrm{um}$ and 1.06um source is demonstrated. Measurement of intraocular distance of 20-30 mm of pig eye and human eye in 2D tomograph was demonstrated to prove the effectiveness of this method. Estimate of spectral linewidth by introducing multimode rate equations are briefly discussed in the end.

In Chapter 4, various configurations to effectively achieve wide range sweep at higher swept range are disclosed. Two techniques are introduced to effectively maximize the gain bandwidth of SOA. The other two approaches are variations to polygon scanner based Littrow grating filter to broaden the Free Spectral Range (FSR) of the tunable filter or higher the Finesse. Swept rate of $50 \mathrm{kHz}$ is demonstrated using this technique.

In Chapter 5, SS-OCT system is described. Configuration and signal process step is briefly disclosed. OCT image samples with various tissues are shown with a brief clinical point of view.

In Chapter 6, feasibility study on single mode hop-free swept lasers is discussed for the future improvement.

In Chapter 7 , concluding remarks are addressed.

The benefits of OCT using a high-speed scanning laser are clear. OCT will significantly reduce the need for surgical biopsy for cancer diagnosis. OCT will transform the diagnosis of retinal diseases such as glaucoma. OCT will be an invaluable tool in treating the occurrence of heart disease and reducing the need for surgery. These are just a few of the many new technologies that will be supported by this new type of high speed scanning laser. Other applications including fiber sensing and spectroscopy will surely follow a similar path taking advantage of this new high speed scanning laser technology. 
Organization of thesis

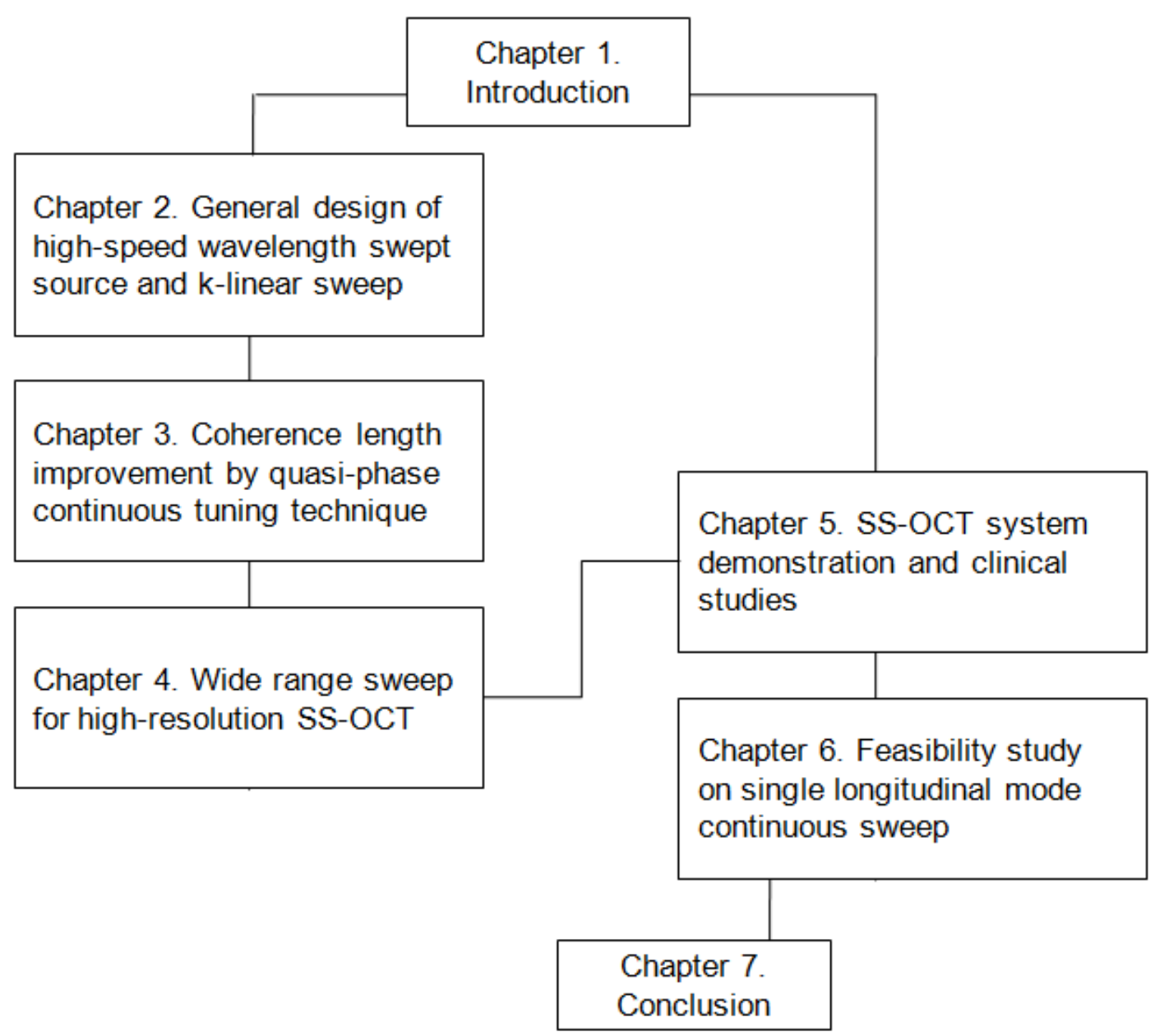

Appendix: SS-OCT image gallery 


\section{Chapter 2 General design of high-speed wavelength swept source and k-linear sweep}

\subsection{Introduction}

Recent studies advocate the sensitivity advantage of frequency domain or swept source OCT(SS-OCT) over conventional time-domain OCT(TD-OCT)[1,2]. In addition the SS-OCT technique can reduce undesirable imaging artifacts. Motion artifacts caused by the presence of pulsation, internal organs motion, or endoscopic motion significantly affect the quality of in vivo images. Utilizing a high-speed imaging or rapid image capturing technique can reduce detrimental effects of motion. This feature has been discussed previously by Yun, et al. [21]. A key component of the SS-OCT technique is the light source; this must be stable, widely tunable and operate at high sweep speeds. Lasers have been identified as the best approach to realize ideal SS-OCT light source, however development of OCT lasers has been slow since first proposed over a decade ago [15]. In this time much of tunable laser development was focused on single mode oscillation and not designed to sweep repetitively, continuously, over wide range at high repetition rate. For example, semiconductor tunable lasers for telecommunication transmitters are too narrow in tuning range and bulky solid-state lasers are unstable in operation.

Several types of swept source specifically designed for OCT have been reported, many are ring cavity-based lasers integrated with a fast scanning filters utilizing either fiber Fabry-Perot tunable filters or diffraction grating with various beam deflection mechanisms [47-55]. At the same time as these swept sources advances there have been many developments of the OCT technique to deal with specific issues such as mirror image cancellation and phase-resolved measurement. Most swept sources are based on quasi-continuous tuning. Lasing and tuning are realized by multi-mode oscillation in extended fiber ring cavities with an integrated scanning filter. As the filter is tuned continuously, a multiple of longitudinal modes in the envelope of the filter gradually shift to the neighboring modes over the gain bandwidth, building up the gain from the ASE noise with the incoming modes and suppressing the passing modes at the same time. Gain medium 
in this case is a semiconductor optical amplifier (SOA). Previous works discussed mechanism of lasing and impact on system performance. As indicated in previous work [49], the faster the speed of wavelength tuning, the wider the instantaneous laser linewidth. This is because the cavity longitudinal modes are stationary with respect to the moving filter window, and the window of filter is moving to next adjacent group of modes at a rate faster than the average cavity lifetime when the sweep rate is over $\mathrm{kHz}$ order. This results in insufficient built-up of the gain and suppression of the passing modes. Numerical analysis to explain the detailed dynamics including nonlinear effects of SOA was previously reported by Bilenca, et al. [60].

Another important aspect in swept lasers is the sweep performance including repeatability, swept range and linearity of sweep. Swept sources that use a scanning Fabry-Perot filter or a diffraction grating with a resonant mirror have a non-linear, sinusoidal temporal change of frequency. In OCT systems, non-linear temporal change of scan frequency necessitates the calibration process in k-space after data acquisition of interference signal. Since data acquisition (DAQ) boards samples data in constant time intervals, this calibration process burden the imaging speed because of the buffering of data for processing, thus hinders real-time display of images. This aspect is drawbacks for high-quality, high-speed imaging in OCT systems.

In this Chapter, we present and discuss a high speed, wide range wavelength-swept laser source that has highly linear sweep. The swept laser is designed in a simple and robust configuration with using a diffraction grating and polygon scanner combined with novel compensation optics. The swept source also incorporates a built-in start photodiode that provides external trigger signal to be fed to DAQ board for each axial scan for synchronization of axial scan and frame. The source has a linear k scan capability taking advantage of high linearity and repeatability of sweep of this laser configuration. The laser generates $15 \mathrm{~mW}$ peak output power with $100 \mathrm{~nm}$ tuning range FWHM and $117 \mathrm{~nm}$ in total range at $20 \mathrm{KHz}$ scanning rate. The performance of the laser is investigated, including the coherence length that restricts the axial measurement range for OCT application. An OCT image is obtained from a system that utilizes this laser source with and without wavelength calibration. 


\subsection{Principle and design}

\subsubsection{Laser Configuration}

Figure 2.1 shows the configuration of the wavelength-swept laser source. The laser is configured with an SOA (COVEGA: BOA1017) as the gain medium and a grating-based scanning filter in the extended cavity. Wavelength tuning is achieved by using a diffraction grating (1312line/mm) and a polygon scanner in Littrow arrangement. A 50\% partial reflector at the other end of the cavity is attached to the GRIN lens collimator from which the output light is extracted. Amplified spontaneous emission within the window of this tunable filter is oscillated in the cavity. Insertion loss of the filter was less than $1.5 \mathrm{~dB}$, and bandwidth of filter is $\sim 0.15 \mathrm{~nm}$ with the beam width $\left(1 / \mathrm{e}^{2}\right)$ of collimator of $0.8 \mathrm{~mm}$. The polygon scanner has 30 facets mirrors to achieve $20 \mathrm{kHz}$ scanning rate at $40,000 \mathrm{rpm}$ and is

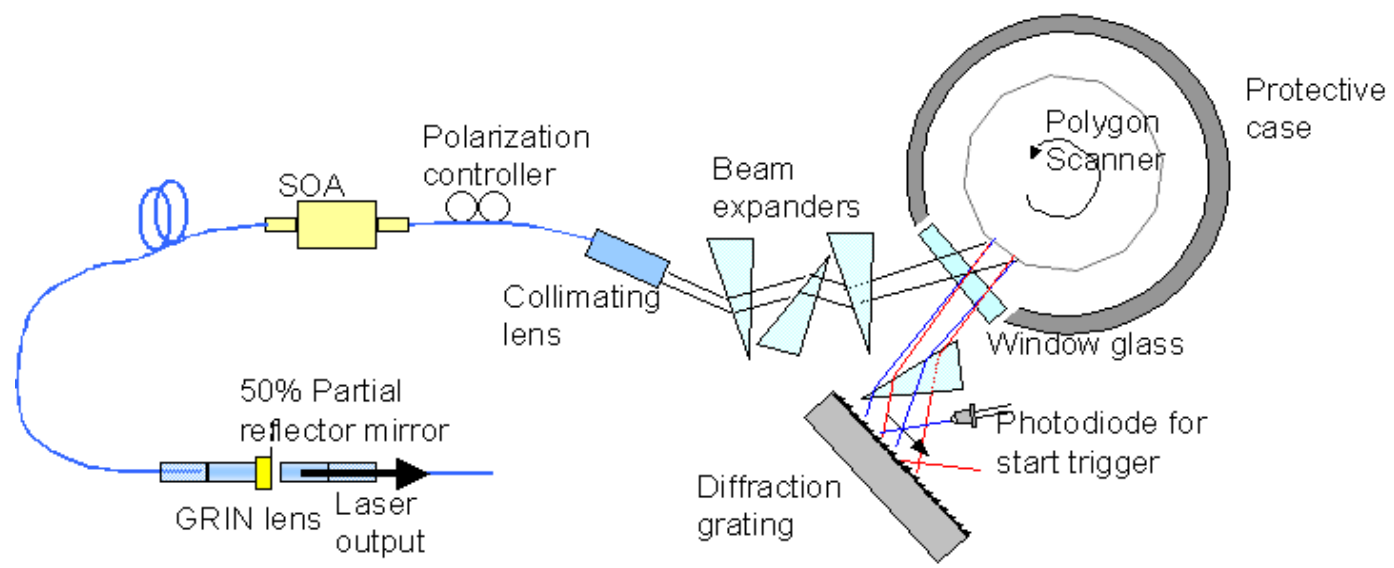

Figure 2.1 Swept laser configuration

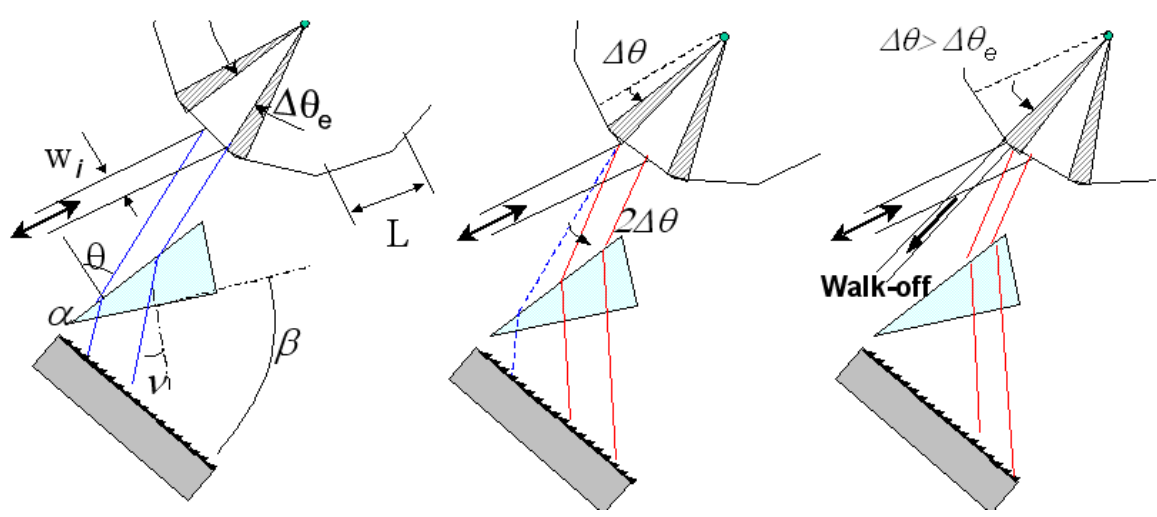

Figure 2.2 Temporal evolution of beam deflection inside the filter block 
sealed inside the protective package with an AR-coated glass window; a dust-absorbing filter is implemented to prevent facet contamination inside the package. The rotational direction of the polygon scanner is chosen to have positive saw-tooth scanning starting from shorter wavelength to longer wavelength since the reverse sweep, high to low wavelength, induces a self-frequency shift due to four-wave mixing by carrier density modulation in the SOA [61,62] lowering laser output power as a results. A scan trigger signal derived from an integrated detector monitoring light in the 0 th order diffraction light path of the grating is provided to enable the start of each scan to be synchronized. Only when the diffracted 0 th order light beam at shorter wavelength intersects the photo detector, does a voltage output arise as a Gaussian response in time. Adding a narrow bandpass filter in front of the detector enhances sharp PD temporal response. The voltage at preset threshold is converted to the TTL output by conversion circuitry integrated in the unit.

\subsubsection{Scanning filter design}

In the scanning filter the polygon scanner continuously deflects the beam to the diffraction grating with a range of angle determined by the number of its facet. This change of angle corresponds to the change of selected wavelength inside the cavity. Longitudinal mode separation is determined by the round trip path length of the cavity, in this case a $4 \mathrm{~m}$ length corresponds to approximately $70 \mathrm{MHz}$ mode spacing. A collimated beam from the SOA is expanded by a series of prism expanders in the plane parallel to the deflection in order to narrow the filter bandwidth, thus narrowing the linewidth of laser output. The total magnification of three prism expanders $\mathrm{M}^{3}$ before the polygon scanner is adjusted to have about a beam diameter as small as $1 / 3$ of polygon facet width. This ensures the effective duty cycle of scanning greater than $2 / 3$ or $65 \%$. Incident angle to the prism expander is set to near the Brewster angle. Setting the prism at Brewster angle minimizes insertion loss because of the grating and prism combination, the diffractive grating having higher diffraction efficiency in $S$ polarization. The other sides of the prisms that have nearly normal incidence is AR coated for additional reduction of insertion loss. The four beam expanding prisms placed in a complementary direction with respect to one after another in terms of refraction of the beam so that the resultant chromatic dispersion of the prism pair is effectively zero. The magnification of single expander $M$ for first three is set to $x 1.5$ by arranging the prism angle $\alpha=30 \mathrm{deg}$, and beam incidence $\theta$ around $60 \mathrm{deg}$ which is near the Brewster angle. The filter bandwidth of diffractive grating is expressed in Eq. (2.1):

$$
\delta v=\frac{c}{2 \pi \omega_{0} \tan (\beta-v)}=\frac{\lambda a \cos (\beta-v)}{\pi w_{0}}
$$

where $\beta$ is the relative angle of the prism with respect to the grating, $v$ is the exit angle of 
the beam from the normal to the prism second facet, and $a$ is the pitch of the grooves of the diffraction grating, or the inverse of grating period $\Lambda$. Provided that the beam width after the polygon mirror is $w_{i}$, then the magnification of the beam after the prism expander $w_{o}$ is given by:

$$
\begin{gathered}
w_{o}=M_{4} \cdot w_{i} \\
M_{4}=\frac{\cos \phi \cdot \cos v}{\cos \theta \cdot \cos \mu}
\end{gathered}
$$

where $\phi$ and $\mu$ are the refracted angle at first and second facet inside the prism. On the other hand, filter wavelength of the diffraction grating in Littrow configuration can be simply expressed as:

$$
\lambda=2 a \sin (\beta-v)
$$

\subsubsection{Sweep linearity}

High linearity of frequency sweep is an important design goal for a scanning laser to be used in OCT investigations. By minimizing the deviation of the laser frequency from a linear extrapolation in time, high-resolution images are obtainable using simple time-constant FFT techniques. Many reported swept lasers have non-linear frequency change over the period of scan. This can be because of mechanical limitations in the tuning element of lasers, such as the sinusoidal drive of a galvanometer. In addition intrinsically non-linear tuning elements, such as diffraction gratings, will result in non-linear scan. For such lasers the instantaneous frequency can only be known by continuous monitoring with an external wavelength or frequency reference. A trigger signal derived from the frequency monitor is used to recalibrate measured OCT interference signals into frequency or k-space before they are sequentially processed to generate images. This process introduces the implementation of an appropriate algorithm for rescaling which may lead to a large task on the data processor and may interrupt the real time imaging capability due to the need of data buffering. Also, this error, the mismatch or inaccuracy of reference to the actual instantaneous frequency results in degradation of signal to noise ratio and resolution. In the case of linear frequency sweep in time, no complicated data rescaling is required, nor the external frequency reference necessary.

The linearity of the laser described in this study is measured to be less than $0.25 \%$ with extremely good repeatability between scans. The high degree of linearity in the scan of the laser is a result of using a prism to compensate for the intrinsic non-linear wavelength selection of the diffraction grating. The polygon scanner used in these investigations has a 
linear rate of beam deflection since the velocity of revolution is precisely locked to a clock-circuit, the deviation of which is less than $0.025 \%$. If the beam is directly incident upon the grating, then diffracted wavelength or frequency is a sinusoidal function of incident angle as in Eq. (2.4). The frequency change is positive parabolic slope over change of angle. On the contrary, the refracted angle of exit beam from prism expander (that equals the incident angle to the grating) has a negative curve of non-linearity in terms of the angle variation. So, in the case that there is a prism arranged in between polygon scanner and grating as shown in Fig.2.2, these two factors may be cancelled out to yield linear variation of frequency over time as a result.

Here, frequency change in terms of deflection angle $\theta$ at polygon scanner is given by:

$$
f(\theta)=c / \lambda=\frac{c}{2 a \sin \left[\beta+\sin ^{-1}\left\{n \cdot \sin \left[\alpha-\sin ^{-1}(\sin \theta / n)\right]\right\}\right]}
$$

Linearity, here, is defined as the deviation of optical frequency from linear extrapolation of a single trace of frequency scan over time. A certain combination of parameters (i.e. $\alpha$ and $\beta$ ) in Eq. (2.5), if appropriately optimized, can reduce the non-linearity of sinusoidal change of wavelength. It is more intuitive to understand the mathematical solution of high linearity though numerically study rather than by analytical derivation. Figure 2.3 shows the linearity with various angles $\beta$ when $\Lambda=1312$ lines $/ \mathrm{mm}$, provided that the required wavelength scan range is fixed at 1260 to $1360 \mathrm{~nm}$. Chromatic dispersion of refractive index of the prism glass (BK7) is also taken into account for the beam refraction in which frequency change as the beam deflects.

As seen in Fig. 2.3, when the deviation from linear extrapolation traces the third order polynomial curve, and the middle flexion point coincides with the center of the frequency range, the linearity reaches near minimum. This is when $\beta$ is approaching to 68.5 degree in the case that $L=1312 \mathrm{lines} / \mathrm{mm}$ and $\alpha=30$ degree. This translates that high linearity solution exists when $d^{3} f(\theta) / d \theta^{3}=0$ around at the center frequency. Figure 2.4 shows the high linearity solutions with different diffraction constants, implicating that smaller constant would yield better compensation thus higher linearity. For an example, when $\Lambda=1000$ lines $/ \mathrm{mm}$ linearity can be minimized to less than $0.05 \%$ while $1312 \mathrm{lines} / \mathrm{mm}$ yields $0.25 \%$ linearity. 


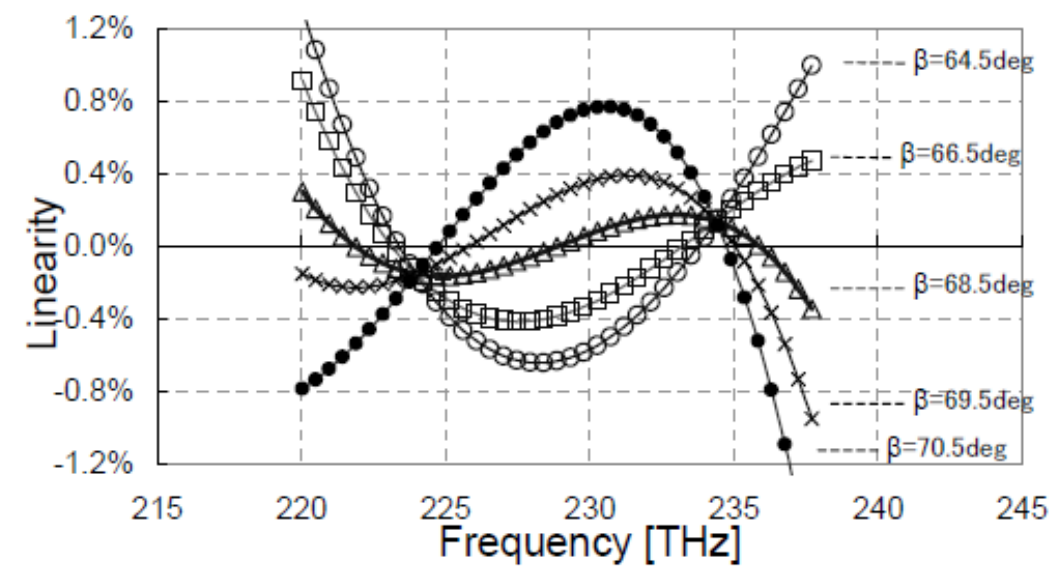

Figure 2.3 Linearity over frequency range with different $\beta$

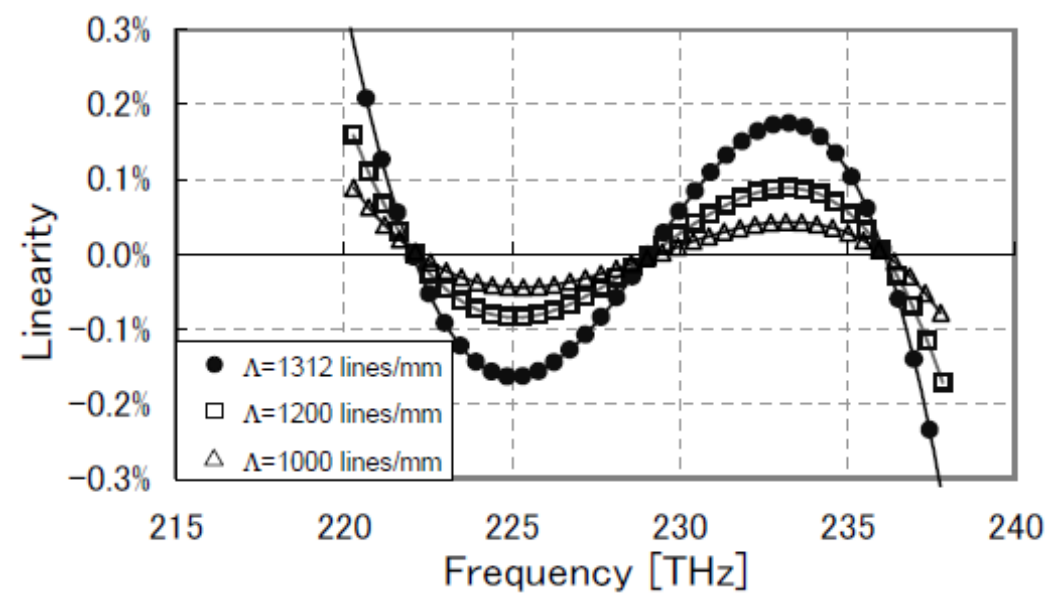

Figure 2.4 Linearity over frequency range with different $\Lambda$ 
In addition to the linearity performance, when the angle $\beta$ is provided in the range close to the calculated value in the above, the filter bandwidth tends to be uniform over the range of incident angle corresponding to the wavelength scanning range. This is because the magnification of the prism expander deceases toward the longer wavelength due to steeper incident angle so that it cancels the narrowing of the filter bandwidth according to Eq. (2.1). This balances the bandwidth over the wavelength range as a result. This uniformity is advantageous performance especially when the source is used for OCT system combined with spectroscopic application or for the other applications that need the spectral parameter encoding such as the fiber sensing system. Figure 2.5 shows the calculated filter bandwidth of diffractive grating with respect to the wavelength scanning range, where parameters are

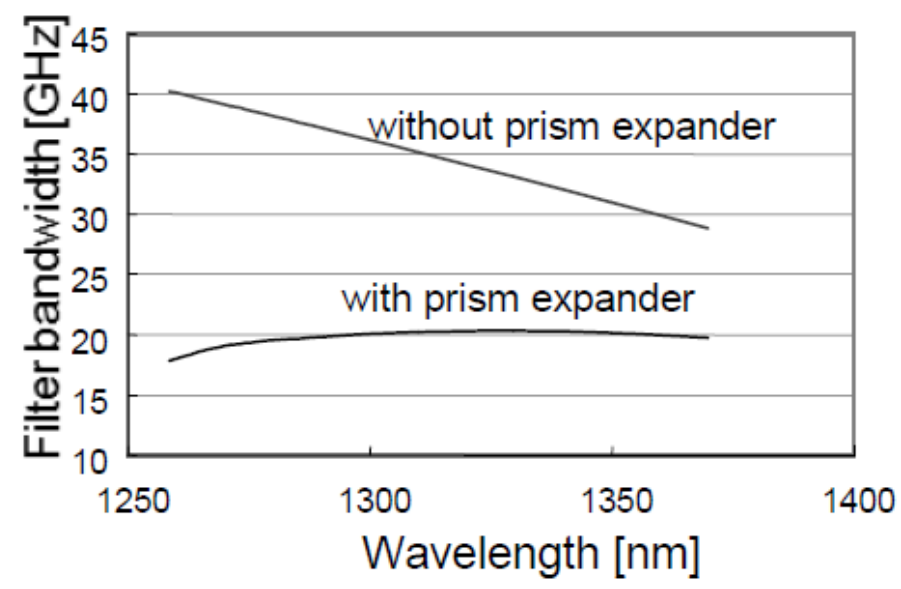

Figure 2.5 Filter bandwidth over wavelength range

set as $\alpha=30 \mathrm{deg}, \beta=68.5 \mathrm{deg}$ and $\Lambda=1312 \mathrm{lines} / \mathrm{mm}$. The filter bandwidth in this arrangement is uniform having a bandwidth of $20 \mathrm{GHz}(0.13 \mathrm{~nm})$ over the entire $1260-1360 \mathrm{~nm}$-tuning range.

Figure 2.6 shows a simulation result of point-spread functions in the case of the ideal linear sampling with no error (IDEAL), and k-linear scan with an error of $0.1 \%, 0.25 \%$ and $0.8 \%$, respectively. Frequency traces are derived from Eq. (2.5) for each case. Simple FFT is performed with Gaussian window on the sinusoidal fringes equivalent to the data sets that can be obtained when the mirror is positioned at $1 \mathrm{~mm}$ depth position in the OCT system. K-linear scan with about $0.25 \%$ linearity exhibits moderate but tolerable axial resolution, and no $\mathrm{S} / \mathrm{N}$ degradation, while $0.8 \%$ shows significant degradation in axial resolution, which is not tolerable in the OCT imaging.

Figure 2.7 shows depth dependence of axial resolution with different linearity values. $0.25 \%$ linearity gives tolerable resolution up to about $1 \mathrm{~mm}$, and $0.1 \%$ linearity gives better 
resolution up to $2 \mathrm{~mm}$ depth range. Choice of other window functions may additionally improve the tolerance against depth range. [63]

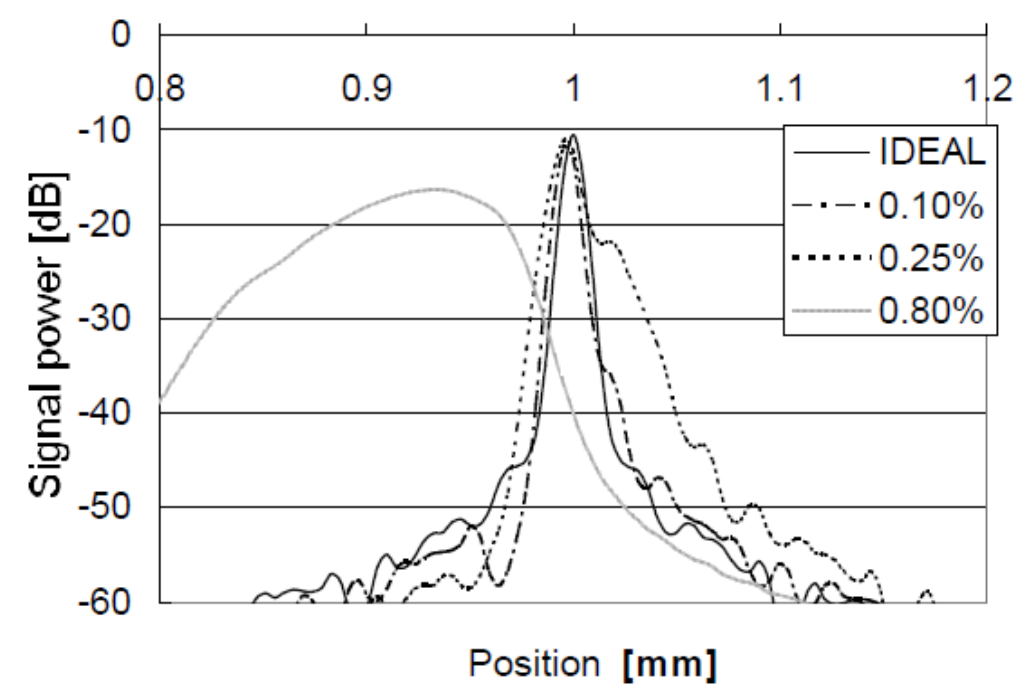

Figure 2.6 Simulated point spread functions (signal spectrum)

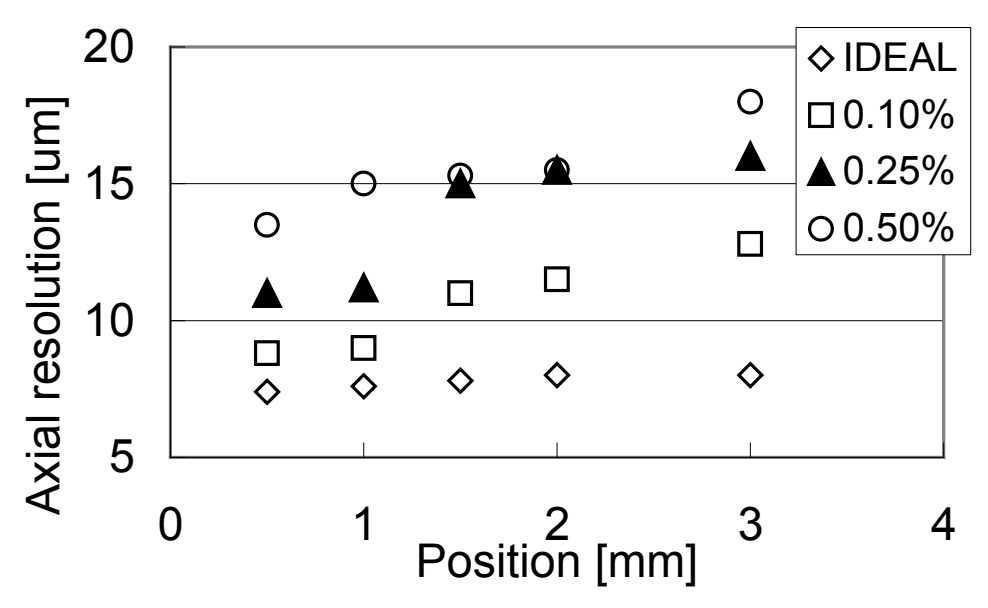

Figure 2.7 Depth dependence of axial resolution 


\subsubsection{Other parameters}

Other important design parameters for the source are discussed in the followings. When the faster scanning rate is desired, the number of polygon facets needs to be increased because the upper rotational speed of polygon scanner is limited. However, this change decreases the FSR of the scanning range because of reduction of the deflection angle as well as so called walk-off issue as depicted in Fig.2.2. Scanning range and rate of the scanning filter considering the above issues are determined by the following equations:

$$
\begin{gathered}
F_{S}=N f_{P} \\
\Delta \lambda_{\text {Littrow }}=4 a \cos \theta_{0} \cdot \Delta \theta_{m} / M \\
\Delta \theta_{e}=720\left(1-\frac{w_{o}}{L}\right) / N
\end{gathered}
$$

where $\theta_{0}$ is the incident angle at center wavelength and $\Delta \theta_{e}$ is effective deflection angle range and $L$ is the facet width of polygon scanner with a diameter, $D, L=\pi D / N$. The term $1-w_{o} / L$ in Eq. (2.8) represents the effective duty cycle of the scanning filter. Polygon scanner is industry-proven technology and suitable adaptation in order to realize repetitive beam scanning at high-speed rate. The polygon scanner of air suspended axis type has a rotation speed ranging from 5,000rpm to 40,000rpm. Scanning rate of a swept source is simply the product of the number of facet $\mathrm{N}$ of the polygon scanner and rotation rate $f_{p}$ that is the inverse of rotation speed as in Eq. (2.6). For an example, the rotation rate $f_{p}$ of the polygon scanner used in this laser is $666.7 \mathrm{~Hz}(40,000 \mathrm{rpm})$, yielding a scan rate of $20 \mathrm{kHz}$ when the number of facet $\mathrm{N}$ is 30 . Eq. (2.7) yields $125 \mathrm{~nm}$ range where parameters are set as $w_{i}=2.7 \mathrm{~mm}, D=60 \mathrm{~mm}$

Trade-off relations of scanning rate and range is shown in the Fig.2.8 according to the Eq.s (2.6), (2.7), (2.8).

When the scanning rate requirement ranges around 5 to $40 \mathrm{kHz}$, diffraction gratings with a grating constant in between 1000 to 13001 ines/mm suffice the scanning range of practical 80 to $100 \mathrm{~nm}$ over which the gain bandwidth of SOA can cover. 


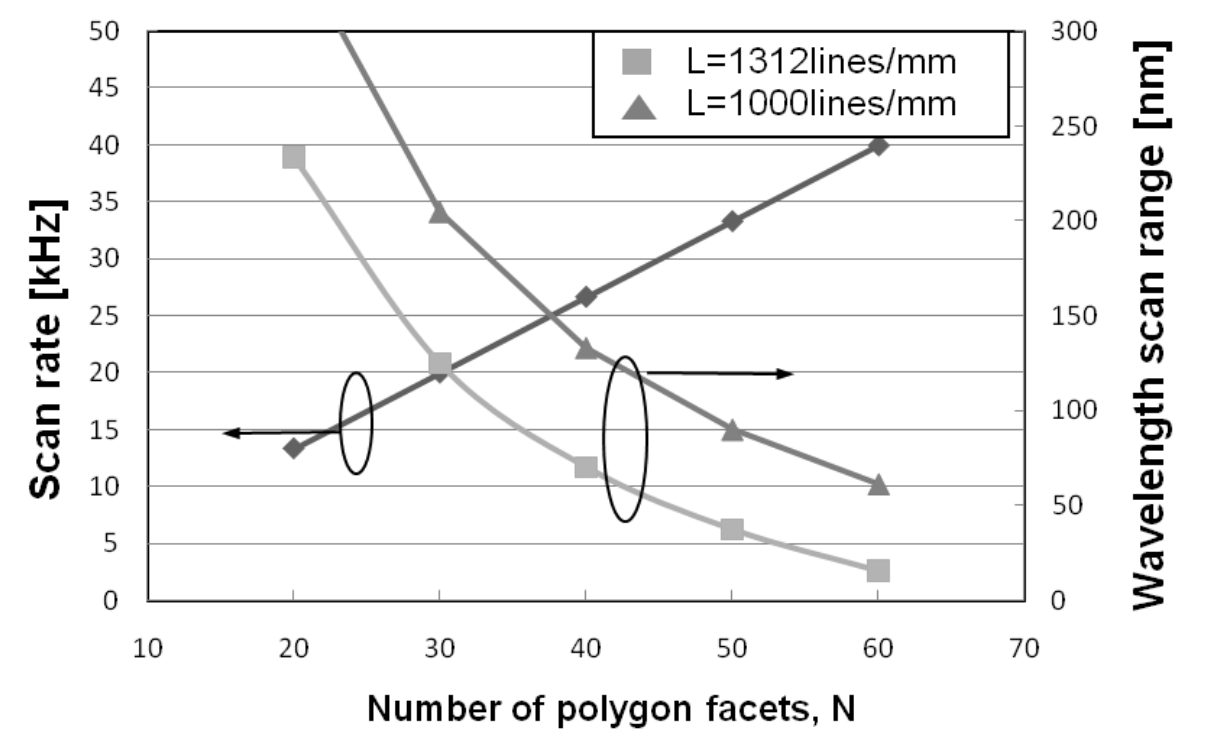

Figure 2.8 Scan rate and scan range vs. the number of polygon facet 


\subsection{Laser performance}

Figure 2.9 shows the temporal laser output power spectrum and a start trigger signal of a fabricated swept source measured with the oscilloscope. The peak and average power were $15 \mathrm{~mW}$ and $9 \mathrm{~mW}$, respectively over the 1267 to $1384 \mathrm{~nm}$ range. Duty cycle is about $75 \%$, which is greater than the expected value of $65 \%$ calculated from the term $1-w_{o} / L$ in Eq. (2.8). This estimation supposes the lasing is on duty when full beam width is contained on the single facet of polygon. $15 \%$ increase of duty cycle indicates that a small portion of beam despite the walk-off transition on the polygon is partially contributed to the lasing. Total scanning range is $117 \mathrm{~nm}$ and FWHM is $100 \mathrm{~nm}$.

In the fiber extended external cavity lasers like our example, laser stability is mainly dependent on two factors; polarization stability and the filter block alignment. In the case of $1.3 \mu \mathrm{m}$ range laser, polarization alignment is relatively insensitive to the stability because of small polarization dependent gain of about $1 \mathrm{~dB}$ or less of the SOA device in $1.3 \mu \mathrm{m}$ range. Figure 2.10 shows the photograph of the fabricated scanning filter part and mechanical drawing of laser source. All the optics is aligned on the metal base plate allowing simple but precise positioning of optical arrangement in a single degree of adjustment axis. Total optical path length of the filter part is only $10 \mathrm{~cm}$ in single path owing to the compact size. This configuration provides not only production robustness but also high reliability of the operation. Measured output power fluctuation were less than $1 \%$ including scan-to-scan variation over a temperature range of 10 to $40 \mathrm{degC}$ over 8 hours. In order to further verify the rigidity of the proposed structure, we have carried out the mechanical integrity test according to Telcordia GR-63 and confirmed the fabricated unit passed the tests including mechanical shock and vibration tests (table2.1). Temporal change of wavelength was measured by counting the fringes from the output of a Michelson interferometer with FSR of $4 \mathrm{~nm}$.

Wavelength jitter of the start trigger was measured to be less than $0.1 \mathrm{~nm}$, and the linearity of tuning in frequency was found less than $0.25 \%$ as shown in Fig. 2.11 , close to the predicted linearity. Further optimization is possible by refining the combination of prism angle and grating pitch. Figure 2.12 shows the relative intensity noise (RIN) of the light source during operation. It had a peak value of $-100 \mathrm{~dB} / \mathrm{Hz}$ at near DC to $4 \mathrm{MHz}$ range, and $-130 / \mathrm{Hz}$ over $20 \mathrm{MHz}$ in between other peaks of about $-100 \mathrm{~dB} / \mathrm{Hz}$ at harmonic frequency of $70 \mathrm{MHz}$ that is resulted from beating of the laser longitudinal mode. 


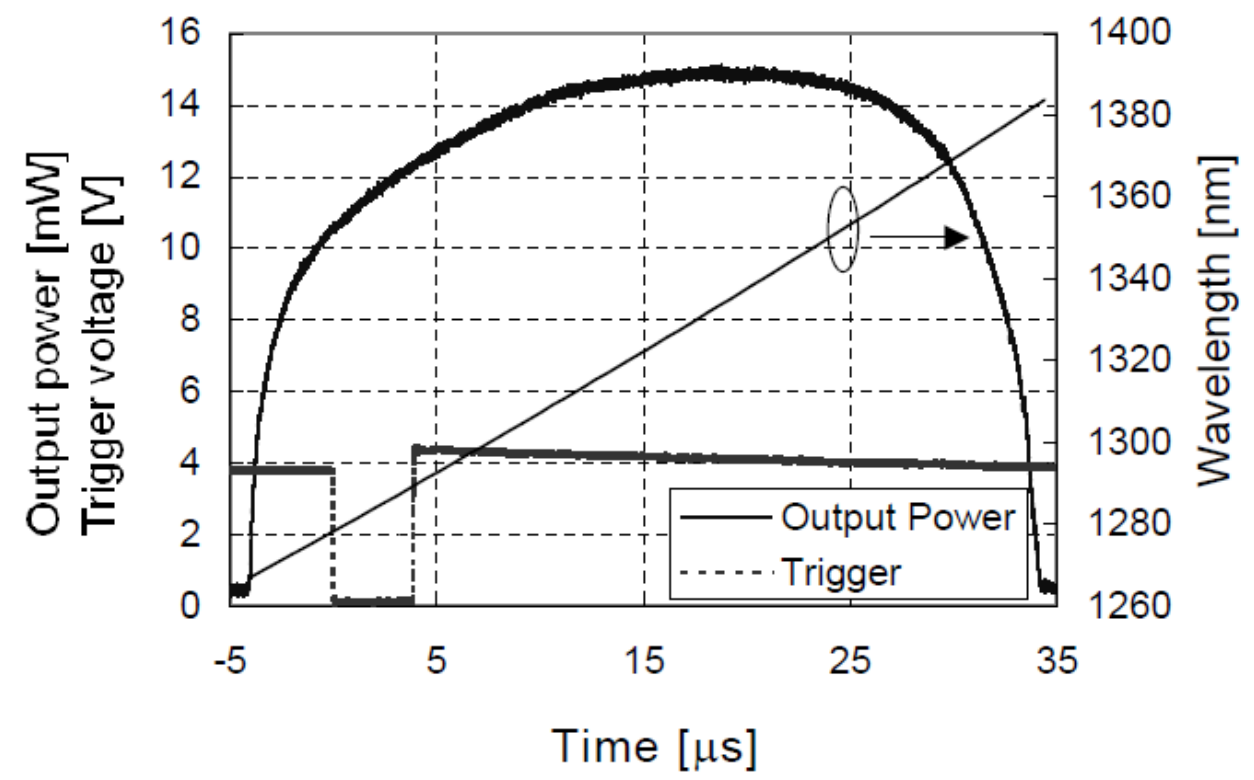

Figure 2.9 Output spectrum of the laser source per scan at $20 \mathrm{kHz}$ scan rate

Table 2.1 Reliability test results

\begin{tabular}{|c|c|c|c|}
\hline Test items & Conditions & Criteria & Pass/Fail \\
\hline Vibration Test & b.3mmp-p amplitude, frequency 10 to $55 \mathrm{~Hz}$ & \multirow{7}{*}{$\begin{array}{l}\text { Max output } \\
\text { power change } \\
<1 \mathrm{~dB} \text { p-p, and } \\
\text { the rest of spec } \\
\text { in spec. }\end{array}$} & Pass \\
\hline Shock test 1 & $\begin{array}{l}\text { side fall: side of the unit is lifted in } 30 \mathrm{deg} \text { from the floor } \\
\text { and dropped in free fall( } 4 \text { sides) }\end{array}$ & & Pass \\
\hline Shock test 2 & $\begin{array}{l}\text { corner fall: side is lifted } 20 \mathrm{~mm} \text { and opposite side of corner } \\
\text { is lifted } 25 \mathrm{~mm} \text { and dropped in free fall( } 4 \text { corners) }\end{array}$ & & Pass \\
\hline Drop test & $\begin{array}{l}\text { When pakced in cardoboard box and fropped from } 80 \mathrm{~cm} \\
\text { height ( } 6 \text { faces) }\end{array}$ & & Pass \\
\hline Temperature range & 10 to $40 \mathrm{deg} C$ & & Pass \\
\hline Temperature cycle & $\begin{array}{l}-20 \mathrm{deg} \text { C } 30 \mathrm{~min} . \Leftrightarrow 60 \mathrm{degC} 30 \mathrm{~min} \text {. (1degC/min slope) } \\
100 \text { cycles }\end{array}$ & & Pass \\
\hline Damp heat test & 60 degC $80 \%$ RH, 100 hours & & Pass \\
\hline Operational Vibration test & $\begin{array}{l}\text { Office vibration (Telcordia GR-63CORE) } 0.1 \mathrm{G}, 5 \text { to } \\
100 \mathrm{~Hz}, 0.1 \text { octave/min. }\end{array}$ & \multirow{2}{*}{$\begin{array}{l}\text { In spec } \\
\text { before/after }\end{array}$} & Pass \\
\hline Operational Vibration test & $\begin{array}{l}\text { Earthquake1 (Telcordia GR-63CORE) 1.6G, } 15 \text { to } 50 \mathrm{~Hz}, 2 \\
\text { octave/min. }\end{array}$ & & Pass \\
\hline
\end{tabular}



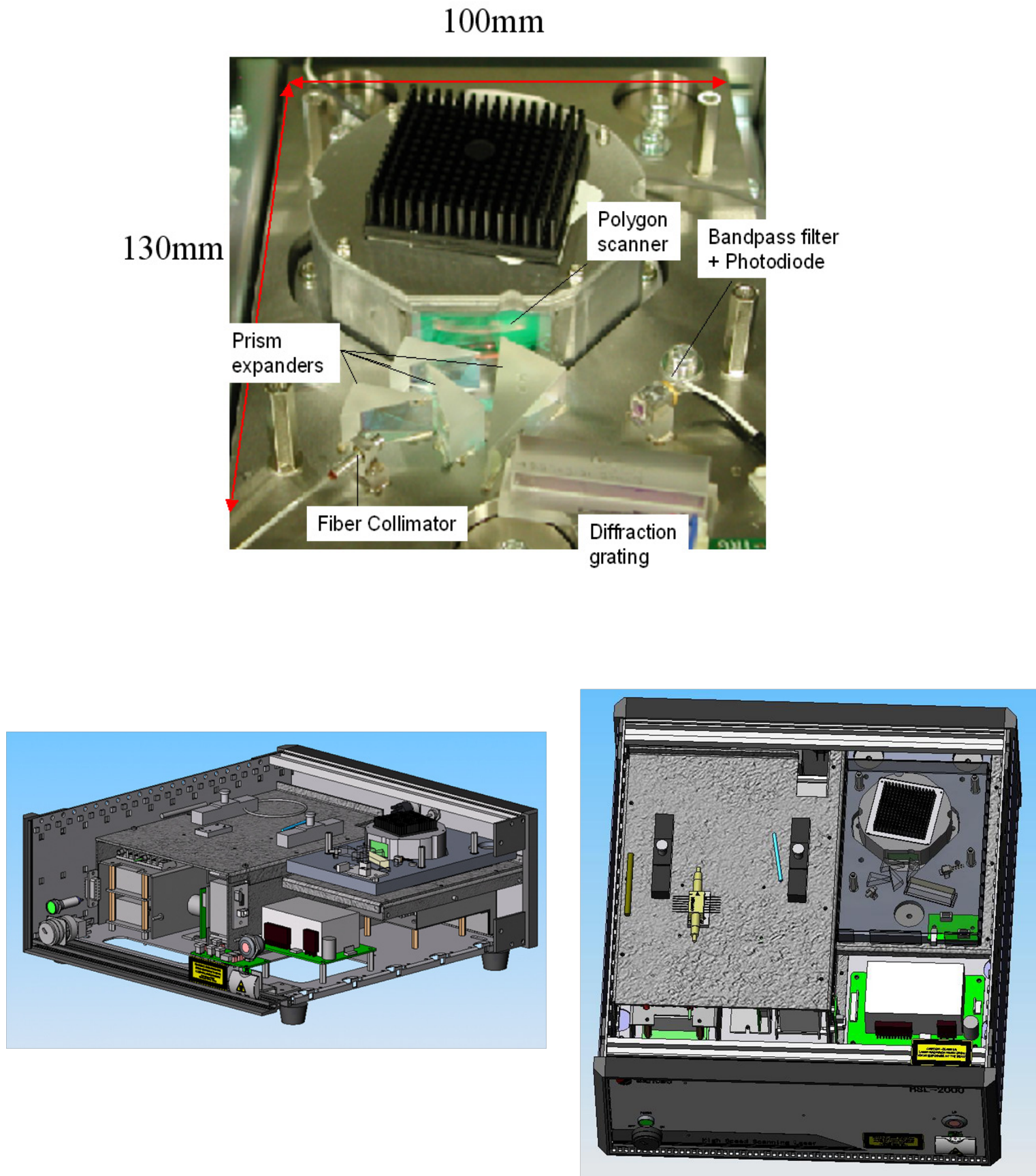

Figure 2.10 Photograph of the scanning filter block (upper) and 3D CAD schematic of fabricated laser source (bottom) 


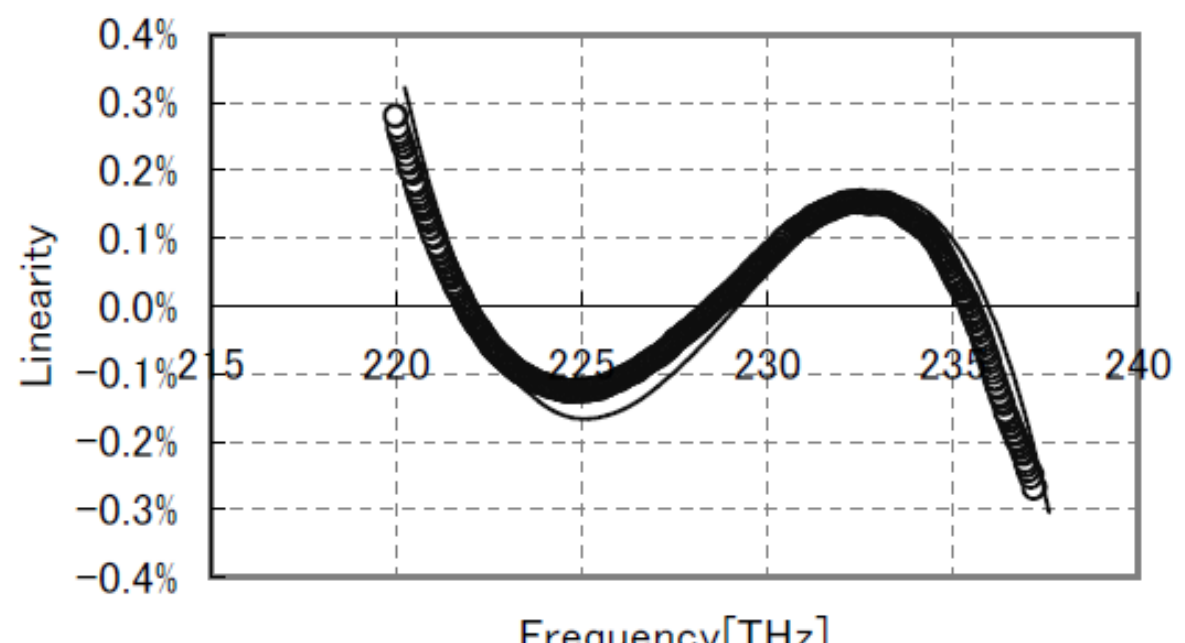

Figure 2.11 Measured linearity of the scan in dots and theoretical line

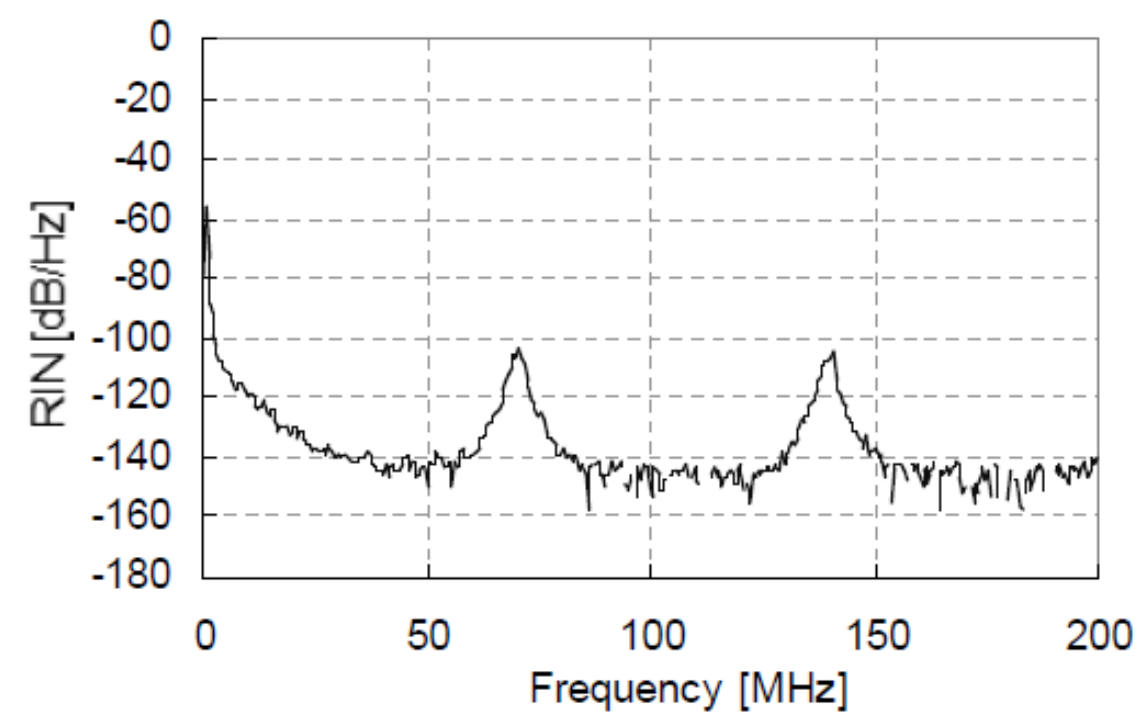

Figure 2.12 Relative intensity noise at $20 \mathrm{KHz}$ scanning rate 


\subsection{OCT System evaluation}

An SS-OCT system with a fabricated swept laser source was configured as shown in Fig.2.13, in order to demonstrate the capability of the swept source in actual OCT images and the effectiveness of its linear sweep. Output beam from the source is introduced into a fiber-based Mach-Zehnder interferometer with a 1:99 1x2 fiber coupler, two circulators, and a 50:50 2x2 fiber coupler. The through port with 90\% coupling ratio of the first coupler is connected to a probe arm though a three-port circulator. Probe arm comprises a collimator lens with a focal length of $10 \mathrm{~mm}$ (Thorlabs.Inc.) and a galvano scanner (Cambridge Technology $6210 \mathrm{H}$ ), achromatic lens with focal length of $100 \mathrm{~mm}$ yielding the lateral resolution of about $25 \mu \mathrm{m}$. The $1 \%$ port of the first coupler is connected to the reference arm which is configured symmetrically with a same circulator, collimator and achromatic lens as those used in the probe arm. Probe and reference beams are recoupled by $2 \times 2$ coupler and two outputs are fed into the balanced photoreceiver (newfocus 1544). A variable optical attenuator is used to adjust the dynamic range of interference signal and optical delay line combined with the objective lens and a reflective mirror is used to adjust the zero delay to match the focus area of the probe arm. An additional visible laser light at $633 \mathrm{~nm}$ is

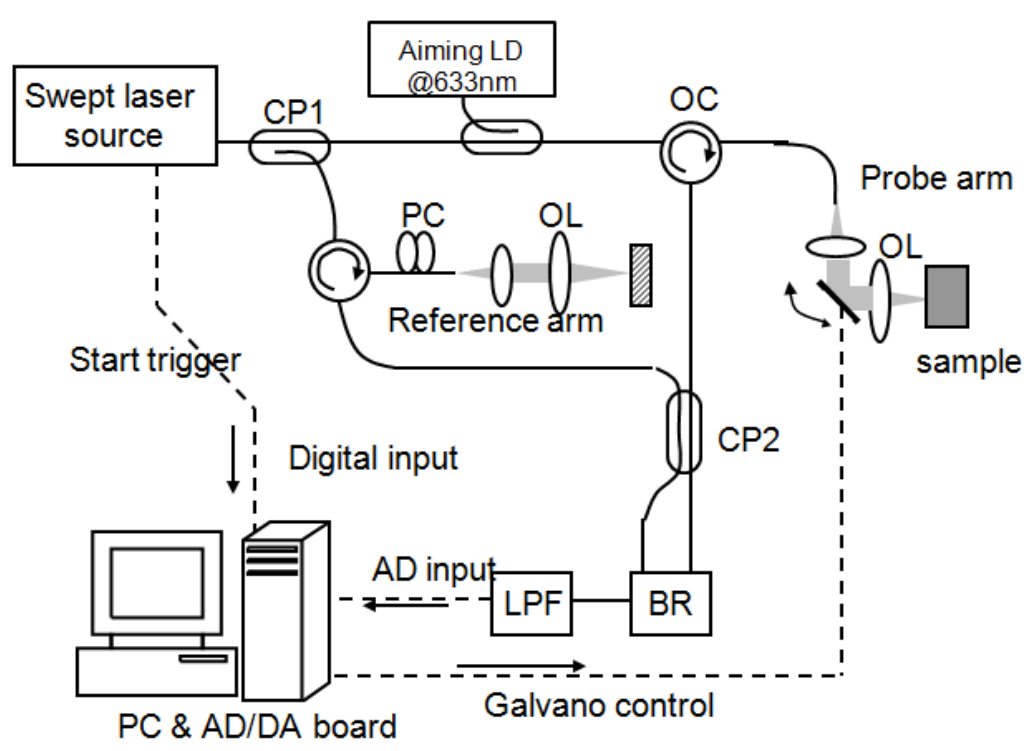

Figure 2.13 SS-OCT system configuration

CP1: 1×2 1:99 fiber coupler, CP2:2×2 50:50 fiber coupler, CP3: 1×2 30:70 fiber coupler, OC: Optical Circulator, PC: Polarization Controller, ATT: Variable Optical Attenuator, OL: Objective lens, G: Galvano scanner, ODL: Optical Delay Line, BR: Balanced Receiver, LPF: Low Pass Filter 
introduced from the reference arm through the fiber coupler and is used for a guide maker on the sample surface in order to make it easy to aim the same target position to be measured. The spectral interference signal is converted to voltage, and digitized by a 14-bit DAQ board (PDA14, Signatec.Inc.) with sampling rate of $100 \mathrm{Msample} / \mathrm{sec}$ that is mounted inside a PC unit.

The start trigger for A-scan synchronization is fed from the integrated start trigger output from the source into the A/D converter. Each start trigger initiates the segmented sampling of about 3500 counts that are transported to the PC, followed by FFT calculation. The start trigger is set to lag about 10-15\% of total scan range behind the actual rise of the lasing start in order to maintain stable detection. Because of this, pre-trigger mode is used in order to make use of $100 \%$ sampling over the effective scan range. Pre-trigger mode is a function in the DAQ board to recall the sampled data prior to the input start trigger using internal buffer on the board. Wavelength rescaling of interference signal is processed in the following steps. First, we obtain an interferogram with using a reflective mirror positioned at $1.5 \mathrm{~mm}$ depth position in order to extract the calibration data, i.e. the time array of peaks and valleys of the interferogram. This time array is then polynominally approximated up to $4^{\text {th }}$ order. By using this data set to rescale the sampled data in actual OCT measurement, the nonlinearity of the temporal frequency change can be properly compensated. Similar rescaling process is discussed in ref. [41]. Signal processing is performed with using LabVIEW program. $512 \times 1024$ pixel image can be output within $0.3 \mathrm{sec}$ including process time for wavelength rescaling. The process time without wavelength rescaling was less than $0.1 \mathrm{sec}$, however, with LabVIEW-based program we cannot accurately estimate the duration of process time because the other programs are running simultaneously consuming PC's memory and run time. We expect the reduction of process time is significant when this is adapted to a programmed FPGA board.

The sensitivity of this SS-OCT is over $100 \mathrm{~dB}$ while the shot-noise limited sensitivity is 119 dB. Figure 2.14 shows the power spectrum at different depth (a) with and (b) without wavelength rescaling process using about $-40 \mathrm{~dB}$ reflector. Signal power drops $6 \mathrm{~dB}$ at around $2.5 \mathrm{~mm}$ in both cases, and no significant degradation of $\mathrm{S} / \mathrm{N}$ is observed even for the case with no rescaling process. The arrangement of the intracavity prism in the source to achieve $0.25 \%$ linearity has a slightly larger beam magnification in comparison with the case of $0.8 \%$ linearity, which results in a narrower linewidth thus a larger coherence length. This may account for the reason why the signal with rescaling process appears to drop slightly faster against depth position than that without rescaling process. The calculated linewidth using the measured coherence length $L_{c}$ from Eq. (2.9) is $22 \mathrm{GHz}$, which is almost equal to the filter bandwidth. 


$$
L c=\frac{2 \ln 2}{\pi} \frac{c}{\delta v}
$$

In the case when wavelength is rescaled by calibration data as shown in Fig.2.15, axial

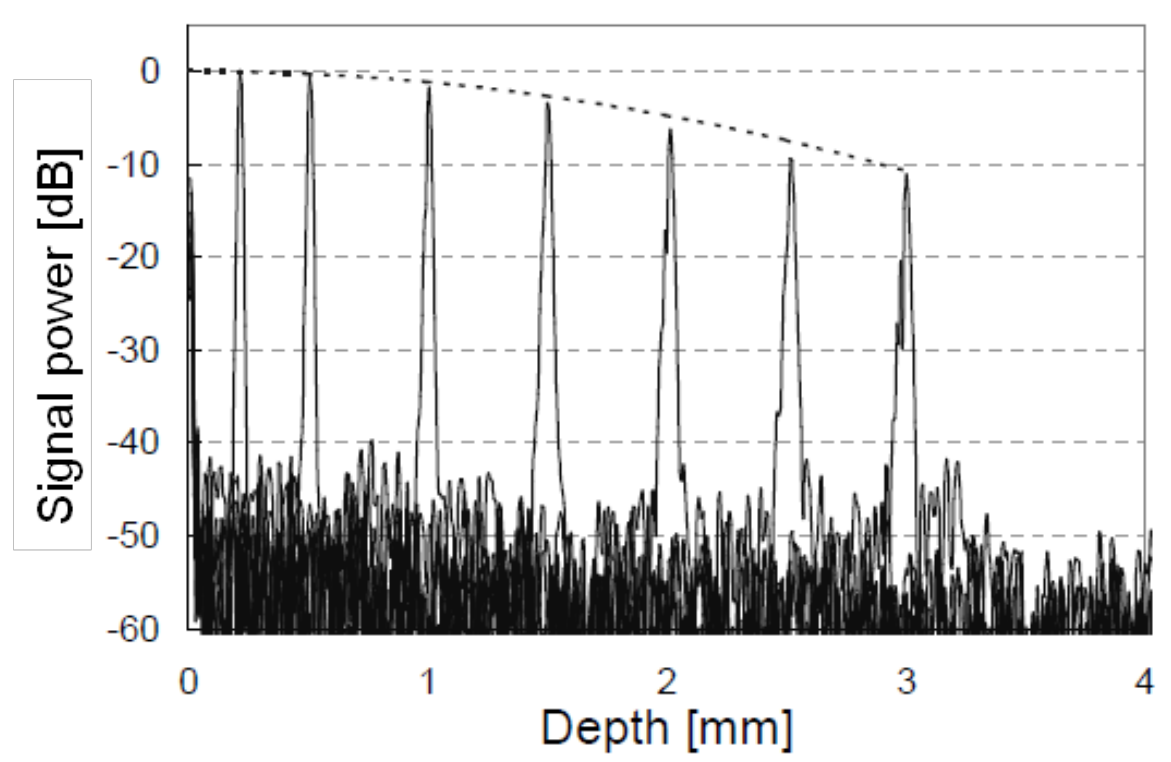

(a) With wavelength rescaling process

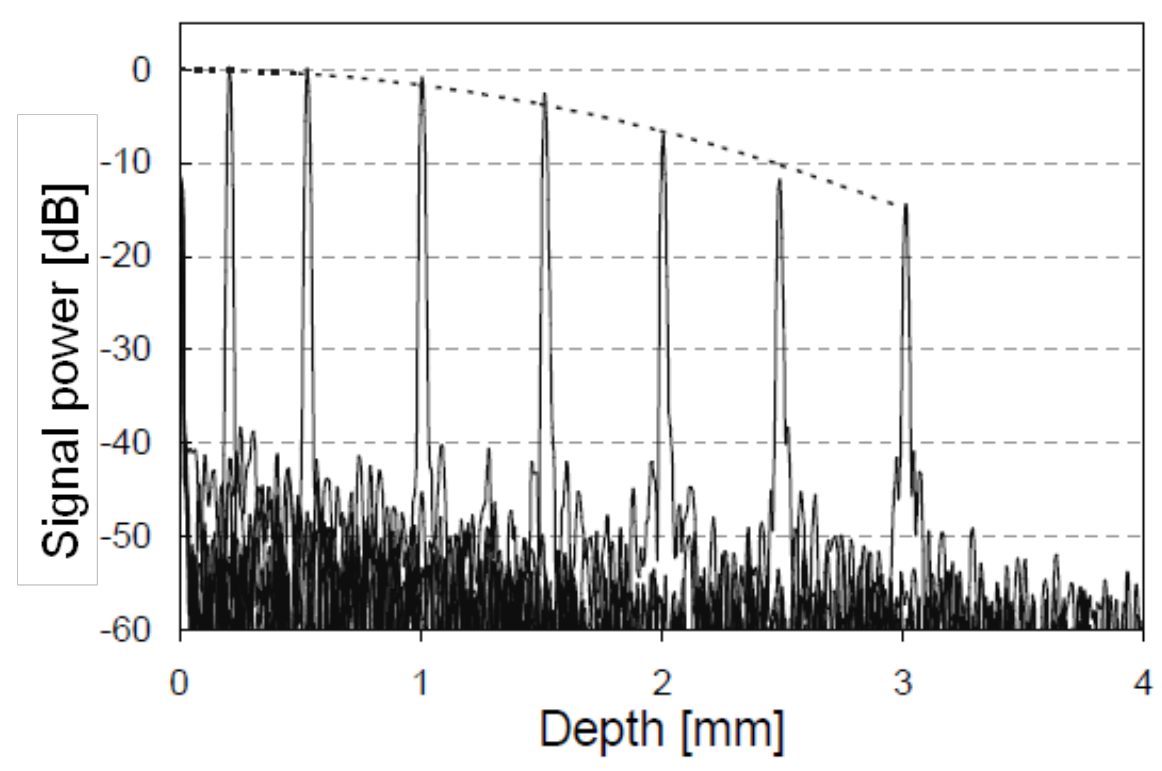

(b) Without wavelength rescaling process

Figure 2.14 Power spectrums

resolution is about $12.5 \mu \mathrm{m}(9 \mu \mathrm{m}$ in tissue) at FWHM in air at the depth of $1 \mathrm{~mm}$, while theoretical value is calculated to $7.7 \mu \mathrm{m}$ in air. Large discrepancy between the theoretical value according to Eq. (2.9) and the measured value may be attributed to the wavelength dependent loss of the components such as optical circulators and collimator lenses used in 
the interferometer. Accumulated loss at the edges of $100 \mathrm{~nm}$ wavelength range was measured about $2 \mathrm{~dB}$ with respect to the center of the range, which narrows the spectral profile of the output power of or the envelope of the interferogram by about $25 \%$. Other calculation errors and noise factors as well as polarization mismatch in the interferometer due to the miss-adjustment of polarization controller may account for additional degradation of axial resolution to some degree.

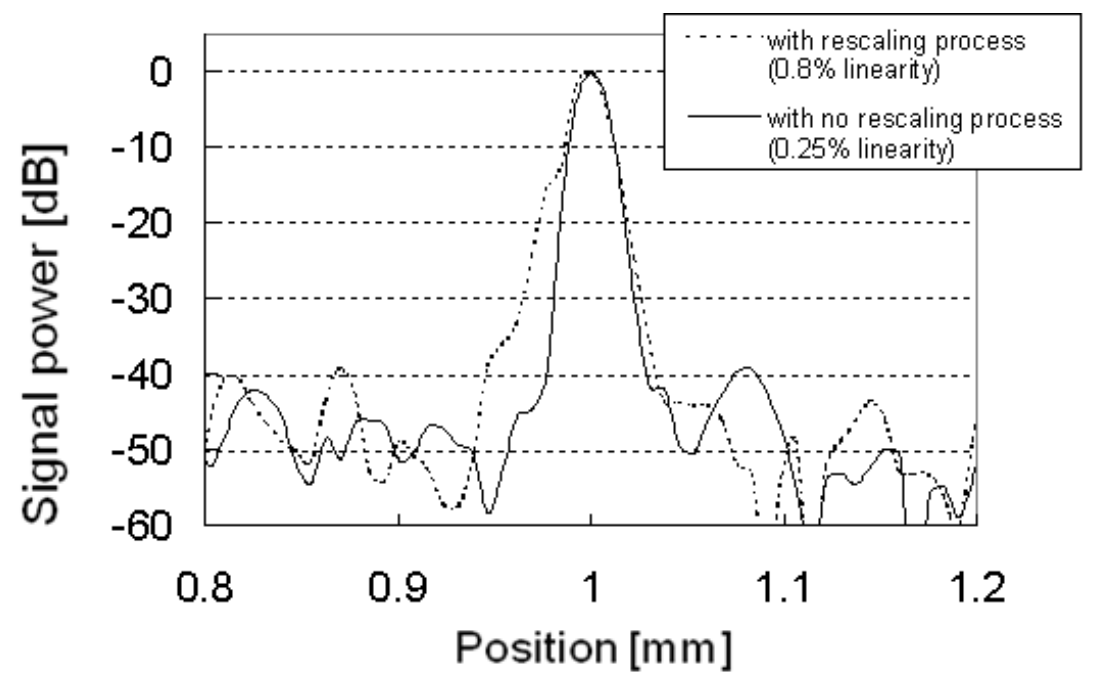

Figure 2.15 Power spectrums at $1 \mathrm{~mm}$

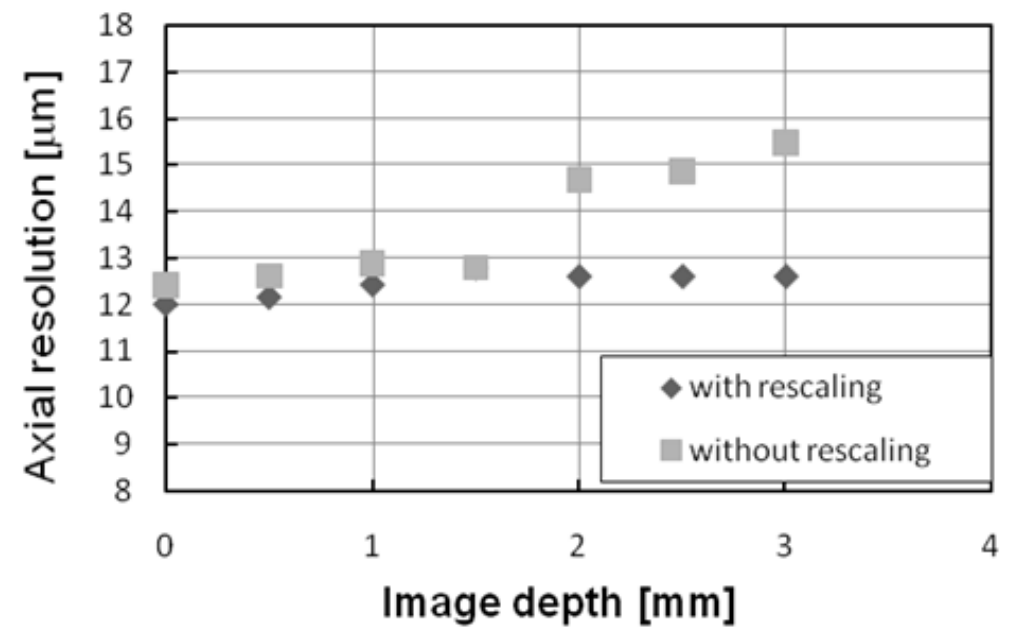

Figure 2.16 Axial resolutions as a function of image depth 
Dashed line shows a spectrum at $1 \mathrm{~mm}$ depth without rescaling process. Axial resolution of this case is about $13 \mu \mathrm{m}$ while the value by previous simulation (in Fig.2.7) was $11 \mu \mathrm{m}$. The wavelength dependent loss of the system and the chromatic dispersion of the interferometer of the system may account for the error in this case. Here, the magnitude of the axial resolution is mostly dominated by the factor of nonlinearity, but not as much by the wavelength dependence as in the case of rescaling process. Spectrum width below $-20 \mathrm{~dB}$ tends to be wider as the depth gets deeper beyond $1 \mathrm{~mm}$ point.

The results indicate that a linearity of frequency sweep as high as 0.1 to $0.3 \%$ can maintain moderate axial resolution as expected and practically eliminate the need of an auxiliary optical k-triggering to be implemented into the OCT system as long as the depth range is relatively shallow about $1-2 \mathrm{~mm}$ range which is practical range of use in the most of OCT applications as shown in Fig.2.16. The previous calculation as in Fig.2.4 suggests that the use of a lower density grating of less than 1100 lines $/ \mathrm{mm}$ is effective to improve the linearity to $0.1 \%$ or less, and which can further improve the axial resolution and $\mathrm{S} / \mathrm{N}$ at deeper range. As proposed in the previous report, the double pass filter configuration may overcome the disadvantage of shorter coherence length when using lower modulation grating that will be discussed in chapter 4 .

In addition, we found that the uniformity of wavelength dependent loss of the interferometer system is another important factor to improve in order to reduce the axial resolution below $10 \mu \mathrm{m}$ order or to reach closer to the theoretical value.

Figure 2.17 shows two-dimensional tomograph of human skin on the finger. Sweat ducts and epidermis are apparent in the images (b), (c). (a) is the image with no wavelength rescaling process with $0.8 \%$ linearity scans. In this case, the broadening of the FFT spectrum blurs the spiral appearance of the sweat ducts and boundary structures such as surfaces of dermis and epidermis. On the other hand, no obvious degradation in the image quality is observed in the case of $0.25 \%$ high linearity scan without rescaling process (b) with a quality comparable to the one with rescaling process with $0.8 \%$ linearity (c). 


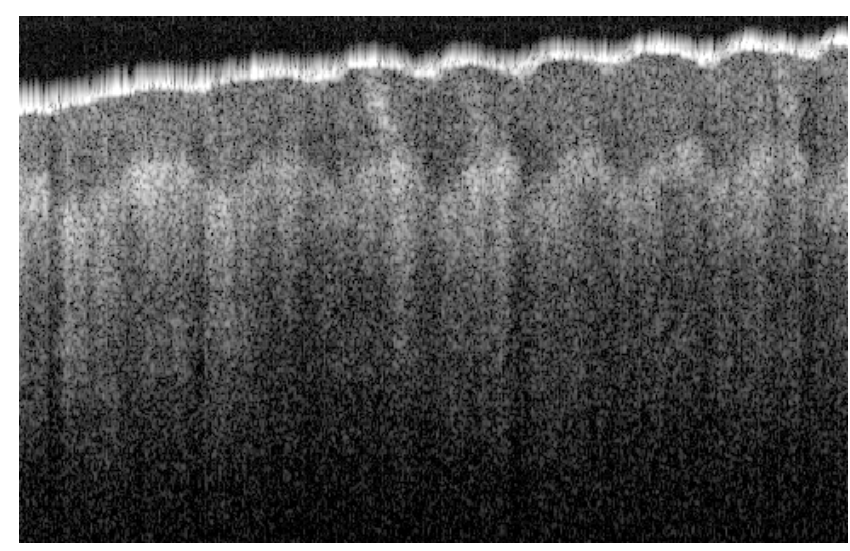

(a)Finger skin image with no wavelength rescaling process (linearity $0.8 \%$ )
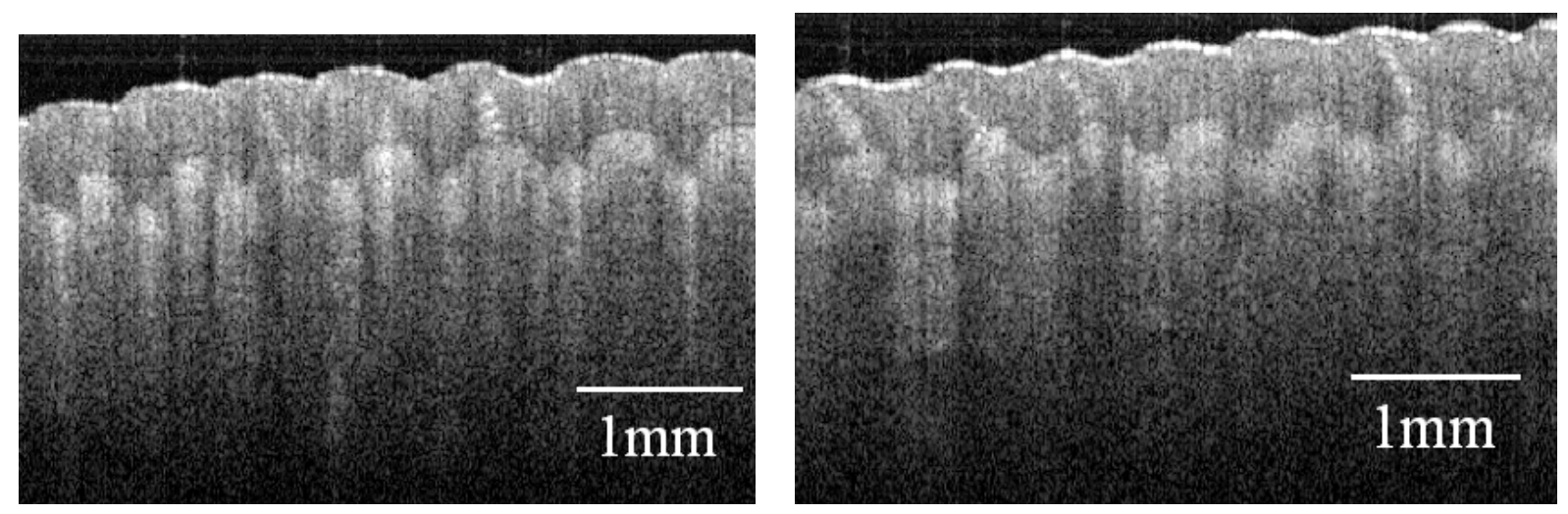

(b) with no wavelength rescaling process(linearity $0.25 \%$ ) (c) with wavelength rescaling process (linearity $0.8 \%$ )

Figure 2.17 OCT image of human finger (Sweat ducts and epidermis) 


\subsection{Summary}

In this chapter, we presented and discussed a high speed, wide range wavelength-swept laser source that has highly linear scan. The laser exhibits $15 \mathrm{~mW}$ peak output power with $100 \mathrm{~nm}$ tuning range in FWHM and $117 \mathrm{~nm}$ in total at $20 \mathrm{kHz}$ scanning rate. The source has k-linear scan capability taking advantages of the high linearity and repeatability of sweep of this laser configuration. Impact of sweep linearity of the source on OCT images was also discussed. In addition to the basic performance of the source, OCT images are also obtained from a system using the fabricated swept laser source, and the effectiveness of linear scan is verified with actual OCT images for the first time.

The simple and robust configuration of the demonstrated laser source should expand the field of OCT in clinical applications in the near future. 


\section{Chapter 3 Coherence length improvement by Quasi-Phase Continuous Tuning (QPCT) technique}

\subsection{Introduction}

Fast wavelength-swept lasers are increasing being used as light sources for applications that require dynamic or real time measurements with high sensitivity and wide dynamic range. Examples include interferometric measurements such as swept-source optical coherence tomography (SS-OCT) and surface profiling, as well as spectroscopic measurements including fiber sensor systems [6], and gas sensing [1-5]. In the case of SS-OCT and surface profiling, coherence length, or instantaneous linewidth of swept source is a key parameters that determines the measurement depth range in cross-sectioned images. In the fiber sensor systems or gas sensing systems, instantaneous linewidth determines the measurement resolution of the system. To maximize the data acquisition rate of such measurement systems it is desirable to increase the scan rate of the swept laser. In general as lasers move to higher sweep speeds there is an increase in instantaneous linewidth, and decrease in coherence length resulting in reduced measurement depth. Typical coherence length previously reported for SS-OCT was limited to 5 to $6 \mathrm{~mm}$ [52-54] corresponding to spectral linewidth of about $0.13 \mathrm{~nm}$ at swept rates ranging from several $\mathrm{kHz}$ to a few tens of $\mathrm{kHz}$. A 5 to $6 \mathrm{~mm}$ coherence length corresponds to the single side measurement depth of 2.5 to $3 \mathrm{~mm}$ in OCT, which has been sufficient for many clinical OCT applications where the depth of interest is limited to the superficial layer and fundamental absorption and scattering in the tissue limits light penetration. However, increase of coherence length is now desired because of following three reasons; 1) demand of increase of the swept rate while maintaining fair amount of depth range, 2) demand of increase of mechanical clearance to the sample surface especially in the case of endoscopic applications so that it can give certain tolerance to the position of the catheter $[11,38,40]$, and 3$)$ the need of larger scale $3 \mathrm{D}$ profiling in industrial applications. Previous work has reported on fiber-extended cavity tunable lasers [24,25,47-49]. In these lasers a tunable filter sweeps over the gain bandwidth of a semiconductor optical amplifier ( $\mathrm{SOA}$ ) and accumulated gain on the cavity longitudinal modes within the envelope of the filter will result in lasing output from the fiber cavity. In 
most cases, cavity longitudinal modes are virtually stationary during the sweep as shown in Fig.3.1. If the tunable filter is stationary or slowly tuned, there is strong cavity mode selection within the filter window resulting in narrow spectral envelope of laser output. On the other hand, if the tunable filter is swept at over a few $\mathrm{kHz}$ rates, cavity mode selection is reduced resulting in an increase output linewidth. The increase in mode competition is due to the mismatch between the modes preferentially amplified in the SOA and the center of the scanning filter over each roundtrip. This is mainly because the most of swept sources are designed to have a fixed cavity length where cavity modes are relatively stationary with respect to the high-speed swept tunable filter.

In order to overcome the increase in output linewidth at higher scan rates, several ideas have been introduced such as the phase matching technique using accousto-optic filter, that matches the wavelength shift over a round trip to the phase shift generated by the filter itself [64]. Another technique is Fourier domain mode locking (FDML), whereby the tunable filter scan frequency is matched to the optical round trip time resulting in a higher $Q$ factor of the cavity in frequency domain [50,65-68]. These two approaches, however, require both to operate at a preset resonant condition, i.e. at fixed swept rate, and the latter case needs long fiber length to accommodate several tens of $\mathrm{kHz}$ swept rate or slower. Other than using these techniques, adding the ambiguity or complex conjugate removal by adding external phase shifter in the OCT system is known as an alternative way [56-59], but it is not preferable when the system design is cost-sensitive.

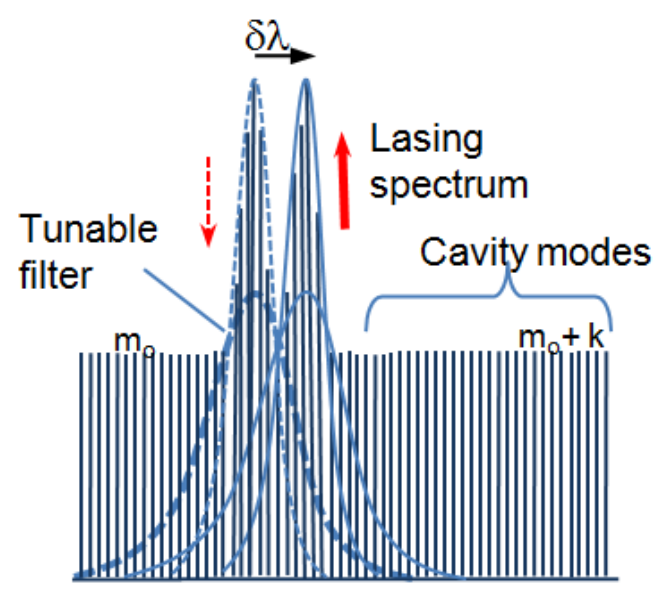

(a) Stationary or slowly tuned case

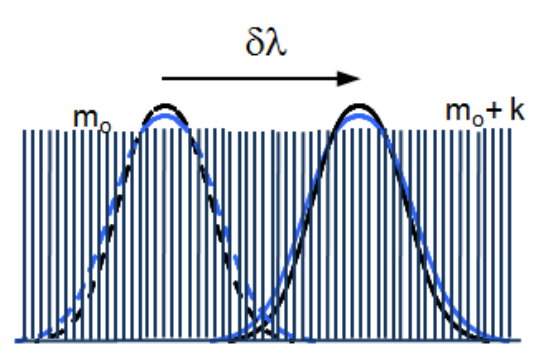

(b) Rapidly swept case

Figure 3.1 Diagram of multi-mode lasing with Gaussian filter envelope

In this study, on the contrary to the above approaches in frequency domain, we demonstrate a rather simple but effective configuration to achieve narrow instantaneous linewidth in time 
domain. We here call this approach Quasi-Phase Continuous Tuning (QPCT) technique, which is analogous to the classical pivot tuning mechanism in external cavity single longitudinal mode laser [69] which we modified and adapted to the fiber-extended cavity.

\subsection{Concept of Quasi-Phase Continuous Tuning (QPCT)}

Figure 3.2 shows the concept of a QPCT laser in comparison with the single mode external cavity laser with perfectly phase-continuous tuning. In a conventional single mode-hop free tuning as shown in Fig.3.2(a), in a simplified case of Littrow arrangement, if the grating is rotated around the so called pivot, the cavity mode and the filter center is completely synchronized over large tuning range without having a mode-hop [69]. This is a novel tuning

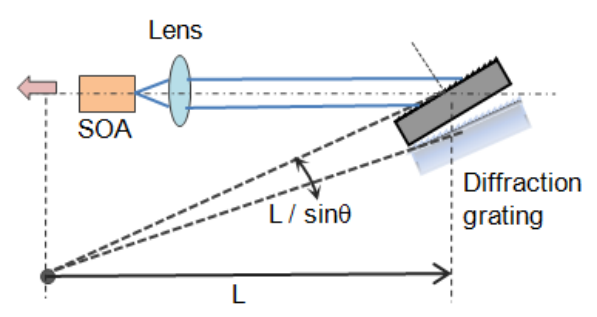

Pivot $(\mathrm{R}, \phi)$

Figure 3.2 (a) Configuration for phase continuous tuning for mode-hop free tuning

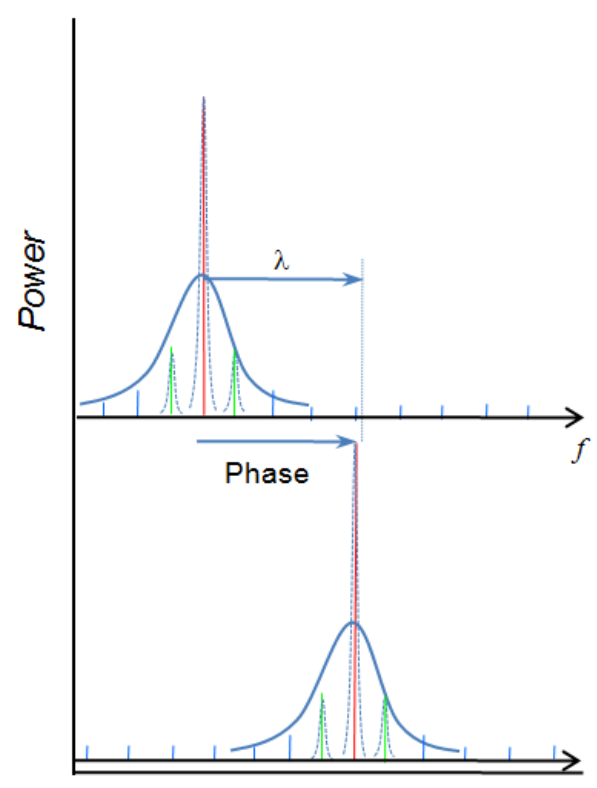

Figure 3.3(a) Phase diagram of Lasing in Fig 2(a)

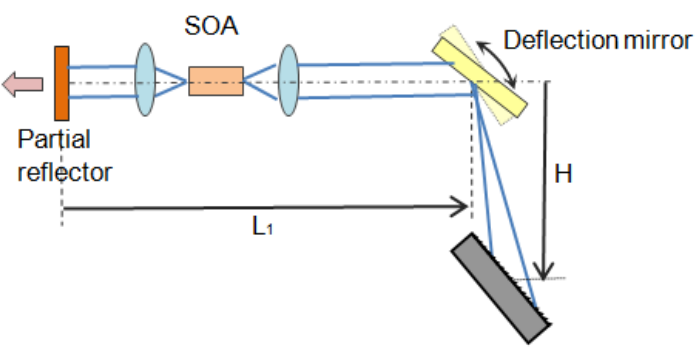

(b) Concept of quasi-phase continuous tuning

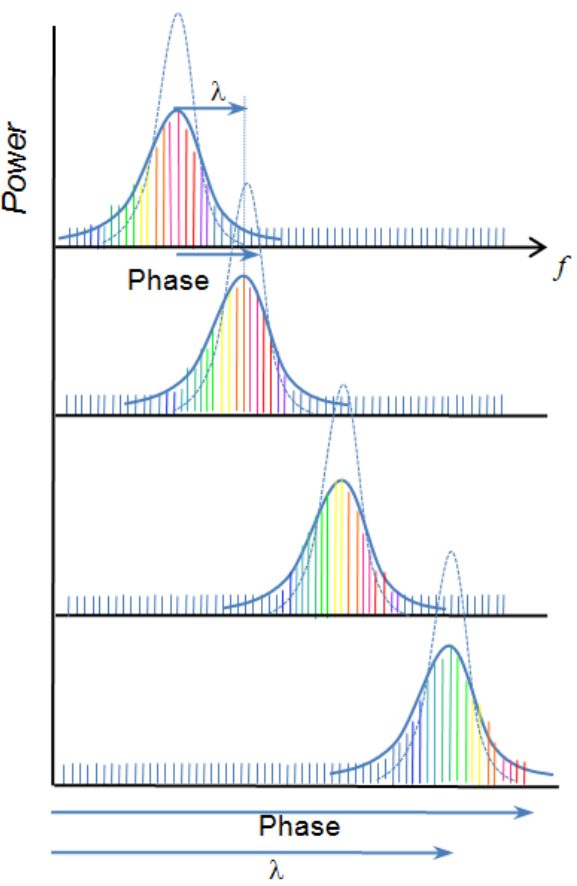

(b) Phase diagram of Lasing in Fig 2(b) 
method because only one rotary motion is required for continuous tuning, and it has been widely applied to the tunable laser instruments. However, the tuning speed or slope is limited to a few seconds per sweep over $100 \mathrm{~nm}$ at 1.3 or $1.55 \mu \mathrm{m}$ wavelength range. This is because the pivot of the rotation is largely off from the optical axis or the point of diffraction, the faster modulation of large momentum is impossible to achieve with today's mechanical actuation technologies. In the case of multi-mode envelope tuning as previously explained, wavelength is tuned continuously over a group of longitudinal modes, gradually shifting to next adjacent modes ensuring continuity of tuning. Figure 3.2 (b) shows the schematic of wavelength swept laser based on QPCT with an SOA and a tunable filter that comprises a diffraction grating and deflection mirror in a Littrow arrangement. Deflection of the collimated beam from the fiber output of the SOA by the scanner mirror changes the incident angle to the diffraction grating thus changes the wavelength of diffracted light couple back to the cavity. At the same time during wavelength sweep, the deflection of the beam modulates the phase by the change of diffraction point on the diffraction grating, which corresponds to the cavity length change. If we set an appropriate geometry so that the gradient of wavelength change and phase are nearly equal, the wavelength and the phase can be synchronously modulated to a certain degree. Figure 3.3 (b) shows the evolution of phase in step by step. Even though the filter's peak wavelength is not perfectly matched to each one of longitudinal mode over entire wavelength range, a majority of longitudinal modes remains in a filter window over the small increment of time allowing a few round trip of light during a scan. As a result, it is expected to enhance the coherent build-up of the laser modes and increase the $\mathrm{Q}$ factor of the cavity during the sweep, which was confirmed in the experiment described in section 4. 


\subsection{Conditions for QPCT}

As previously mentioned, the relation of gradient of wavelength and the phase change simply determines the condition for quasi-phase continuous tuning. Here, total cavity length $L$ is a function of grating incident angle $\theta$, which is expressed in the sum of the optical length from the deflection point to the grating, $H / \cos \theta$ and the length to the other end of cavity, $L_{1}$, thus $L=L_{1}+H / \cos \theta$

Wavelength of the filter, $\lambda_{\mathrm{g}}$ and the wavelength of the cavity longitudinal mode, $\lambda_{\mathrm{L}}$ at the center of wavelength range are expressed in:

$$
\begin{gathered}
\lambda g=2 a \sin \theta \\
\lambda_{L}=\frac{L(\theta)}{L o} \lambda_{L}\left(\theta_{c}\right)
\end{gathered}
$$

$L_{o}, \theta_{c}, a$ are the length and the diffraction angle at the center of wavelength range, and grating pitch, respectively. Then the gradients of these Eqs.s are expressed in (3.3), (3.4)

$$
\begin{gathered}
\lambda_{g}^{\prime}=d \lambda_{g} / d \theta=2 a \cos \theta_{c} \\
d \lambda_{L} / d \theta=\lambda_{c} H \tan \theta_{c} /\left(L_{o} \cos \theta_{c}+H\right)
\end{gathered}
$$

, and are set to equal, then following relation of geometry is derived.

$$
H=\frac{\cos \theta_{c}}{\tan ^{2} \theta_{c}-1} \cdot L_{1}
$$

This is a basic condition for QPCT design, where $\theta_{c}=\operatorname{asin}\left(\lambda_{c} / 2 a\right)$.

Figure 3.4 shows the ratio of $H / L_{l}$ with various diffraction grating constants $(\Lambda(=1 / a)[\mathrm{g} / \mathrm{mm}])$ and with respect to the center wavelength of the tuning range. This graph indicates that depending on the selection of the grating constants $\Lambda$, appropriate ratio of lengths around the point of deflection, $\mathrm{H} / \mathrm{L}_{1}$ has a large variation with respect to the desired wavelength range, and also shows that the lower the wavelength range is such as $1050 \mathrm{~nm}$ range, the higher the grating constant should be chosen in order to fulfill the condition. Figure 3.5(a) shows an example of wavelength and phase trace when the above condition is fulfilled. Figure 3.5(b) shows the cavity mode order $n$ that coincides with filter's peak wavelength over tuning range. In terms of value $R$ as in reference [72], which is defined as the relative frequency change of the cavity modes with respect to the free spectral range during a single roundtrip, $R$ is substantially small. This mode of operation is completely opposite approach of modeless operation as described in ref. [72]. 
We can quantify the degree of phase synchronization in terms of temporal phase lag between longitudinal modes and the filter's peak wavelength over entire wavelength range as the difference of gradients;

$$
P=\frac{d \lambda_{g}-d \lambda_{L}}{d \theta}
$$

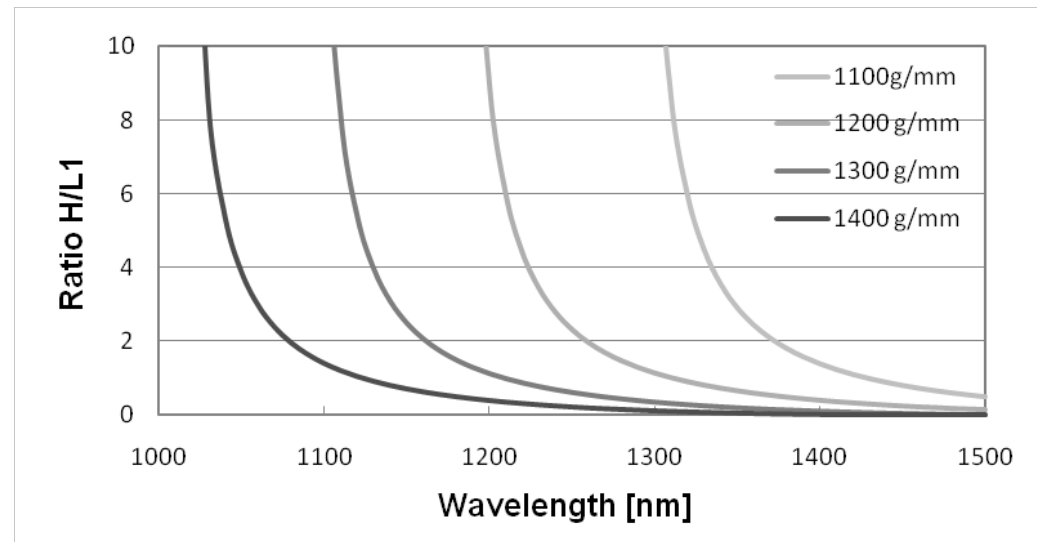

Figure 3.4 QPCT conditions with different wavelength ranges
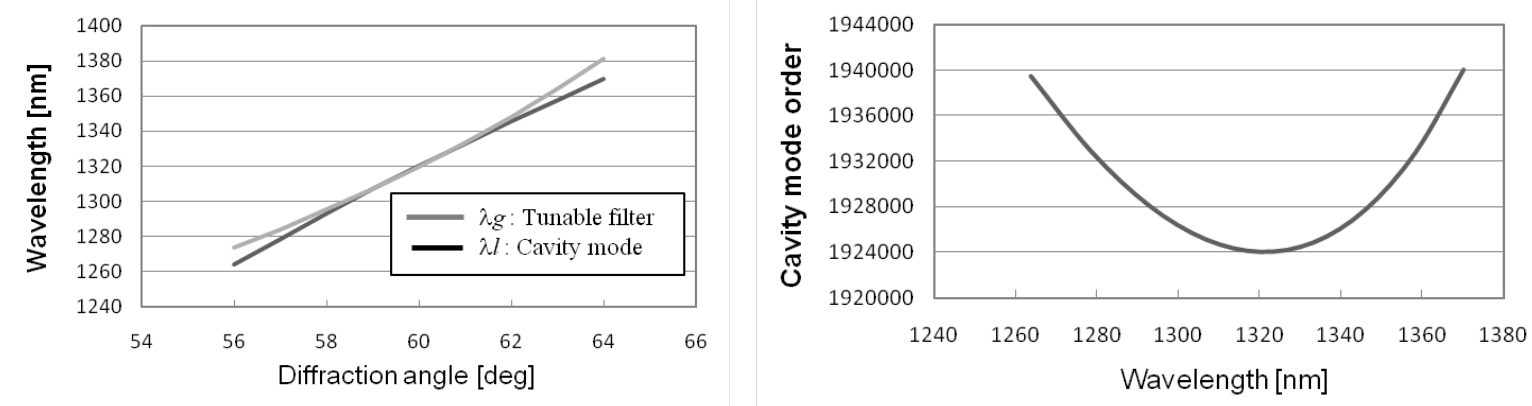

Figure 3.5 (a). Phase variation over diffraction angle range

(b). Cavity mode order over wavelength

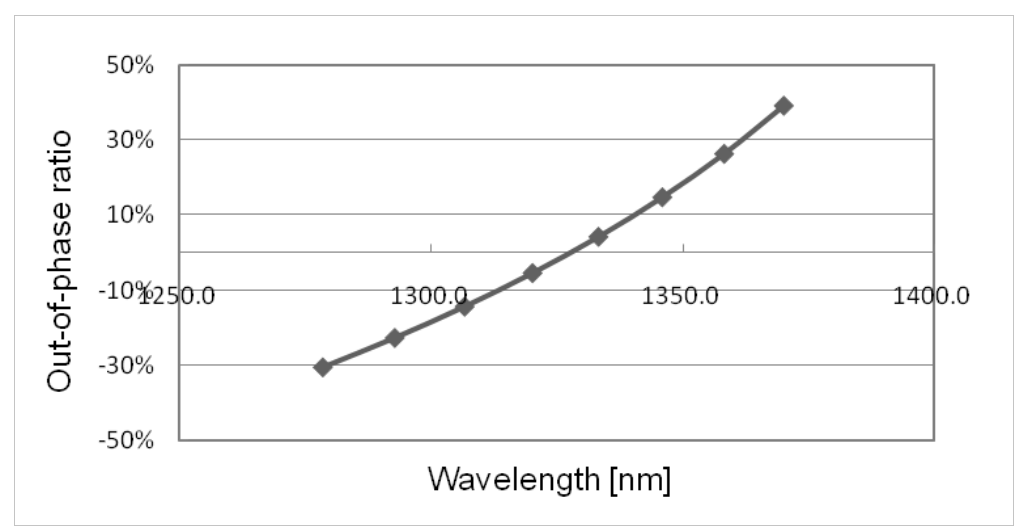

Figure 3.6 Out-of-phase ratio $\mathrm{P}$ over wavelength range 
Figure 3.6 shows the factor of phase lag, $P$ over wavelength range with grating constant of $1312 \mathrm{~g} / \mathrm{mm}$. As shown in Fig. 3.6, the calculated phase lag over small increment of wavelength change is at worst $40 \%$ at the edges of tuning range, in the other words, $60 \%$ are synchronous resulting in a high cavity resonance leading to narrow linewidth of the source. For an example, for an optical cavity length of $3 \mathrm{~m}$ corresponding to a cavity round trip time of $10 \mathrm{nsec}$, and the filter tuning $100 \mathrm{~nm}$ at $20 \mathrm{kHz}$, the tuning slope is $2 \mathrm{~nm} / \mu \mathrm{sec}$. As described in previous work [49], when using an SOA with ASE power of a few milliwatts and over $20 \mathrm{~dB}$ gain and a tunable filter with 2-3 dB insertion loss, it takes about three round trips to build up to the saturated optical output. During three round trips, i.e. 30 nsec, filter wavelength shifts by $0.06 \mathrm{~nm}$, which is nearly a half of filter window. In the other words, if the filter window shifts about a single increment of window in the amount of filter bandwidth; $0.12 \mathrm{~nm}$, there will be no contribution of any preceding cavity modes from the previous window of the filter to a next increment of window. On the other hand, in the case of QPCT, there is always over $70 \%$ of phase or cavity modes carried over from previous round or window of oscillation as it travels the cavity contributing to the build up of the gain, resulting in narrower linewidth. It is equivalent to the slowing the tuning speed of the tunable filter with respect to the cavity modes.

Cavity length is another important factor in order to improve the resonance of the cavity. The shorter the cavity length is, the higher the gain builds up on lasing cavity modes during the high-speed sweep, by allowing many roundtrips within the filter's moving window. However the shortest cavity length is limited by the continuity of sweep. If it is too short, unstable mode competition or bistability within a small number of cavity modes within the window results in unstable or noisy temporal optical power profile. In order to have a continuous and stable sweep of wavelength, a few tens of centimeters to a few meter long cavity lengths is considered appropriate. In this experiment, since the use of single mode fiber instead of free space makes it much easier to configure the laser cavity in terms of the alignment and handling, a pigtailed SOA is used as a gain element which is connected to the tunable filter via a collimator lens and terminated with a partial reflection mirror on the other side of fiber pigtail to form an extended cavity structure as shown in Fig. 3.7. The shortest fiber length that we can prepare for the pigtail part was $0.6 \mathrm{~m}$ from end to end corresponding to optical length $\mathrm{L}_{1}$ of $0.85 \mathrm{~m}$. To have the length of $\mathrm{H}$ in practical range of a couple of tens of centimeters for loss-less free-space coupling by the collimator, we selected a diffraction grating constant of $1312 \mathrm{~g} / \mathrm{mm}$ according to the Eq. (3.5) that gives sufficient synchronization over the tunable range considering the practical length that can be achieved by fiber splicing. From the Fig. 3.4 or Eq. (3.5), the condition for the quasi-phase continuous tuning is found to be when $\mathrm{H}=100 \mathrm{~mm}$. Total cavity length at center wavelength at $1310 \mathrm{~nm}$ is calculated to $2.5 \mathrm{~m}$ per round trip optical path that corresponds to the photon 
lifetime in the cavity of $8.3 \mathrm{nsec}$. In this case, the number of longitudinal modes is about 150 within the window of $0.1 \mathrm{~nm}$ bandwidth of the filter.

Additionally, Doppler shift may arise as a concern when the cavity length modulation is as large as $100 \mathrm{~mm}$ in this case. When swept rate is at $20 \mathrm{kHz}$, the rate of cavity modulation $v_{f}$ is $100 \mathrm{~mm} / 50 \mu \mathrm{sec}=2,000 \mathrm{~m} / \mathrm{sec}$, and the Doppler shift calculated by $\Delta f_{\text {Doppler }}=f /\left(c+v_{f}\right)$ is about $1.5 \mathrm{GHz}$ in optical frequency or $9 \mathrm{pm}$ in wavelength. This is negligible magnitude because the spectral linewidth of interest in this study is in the order of 0.05 to $0.1 \mathrm{~nm}$. However, either when further lienwidth narrowing is necessary to the order equal to the Doppler shift, or when increasing the swept rate resulting in larger Doppler shift, the Doppler shift becomes non-negligible order to the linewidth, and this needs to be accounted for the design. One way to countermeasure this problem is either scaling down the cavity length or choosing the condition of $\mathrm{H}<<\mathrm{L}_{1}$.

\subsection{Experimental setup}

Figure 3.7 shows the schematic of the QPCT laser that was used to verify the proof-of-concept. The laser comprises an SOA (COVEGA BOA1137) and a polygon scanner based grating filter in a Littrow arrangement. Polygon mirror has an inscribed diameter of $60 \mathrm{~mm}$ with 30 facets, and its rotation speed is set at 40,000 rpm for $20 \mathrm{kHz}$ swept rate and $5,000 \mathrm{rpm}$ for $2.5 \mathrm{kHz}$ rate. The rotational direction of the polygon scanner is chosen to have positive saw-tooth scanning starting from shorter to longer wavelength since the reverse sweep induces a self-frequency shift due to four-wave mixing by carrier density modulation in the SOA $[61,62]$ lowering laser output power and also widening the spectral linewidth as a results. Collimated beam with a beam diameter $\left(2 w\right.$ at $\left.1 / \mathrm{e}^{2}\right)$ of $800 \mu \mathrm{m}$ is expanded to $2 \mathrm{~mm}$ in lateral direction before the polygon mirror by three prism expanders. Polygon facet width of $6.3 \mathrm{~mm}$ is wide enough so that there is no clipping of the beam within effective FSR of the tunable filter. Polarization controllers at the two arms of fiber pigtail are used to match polarization states of the light travelling back and forwards with respect to the diffraction grating and SOA so that the collimate beam is aligned in S-polarization in free-space before the grating, and input and output beam going into the SOA is aligned in TE mode. Modulation constant of the $1312 \mathrm{~g} / \mathrm{mm}$ is chosen to tune over $100 \mathrm{~nm}$ wavelength range around $1320 \mathrm{~nm}$. FSR of tunable filter is $180 \mathrm{~nm}$. The bandwidth of tunable filter is calculated to $0.13 \mathrm{~nm}$. Tunable range is limited by the physical size of the diffraction grating that should cover the effective deflection angle of the beam at the distance $H$. The prism expander between polygon scanner and the diffraction grating is arranged in a way such that the linearity of sweep slope becomes higher, eliminating the need of so called k-triggering and wavelength rescaling process [41]. 


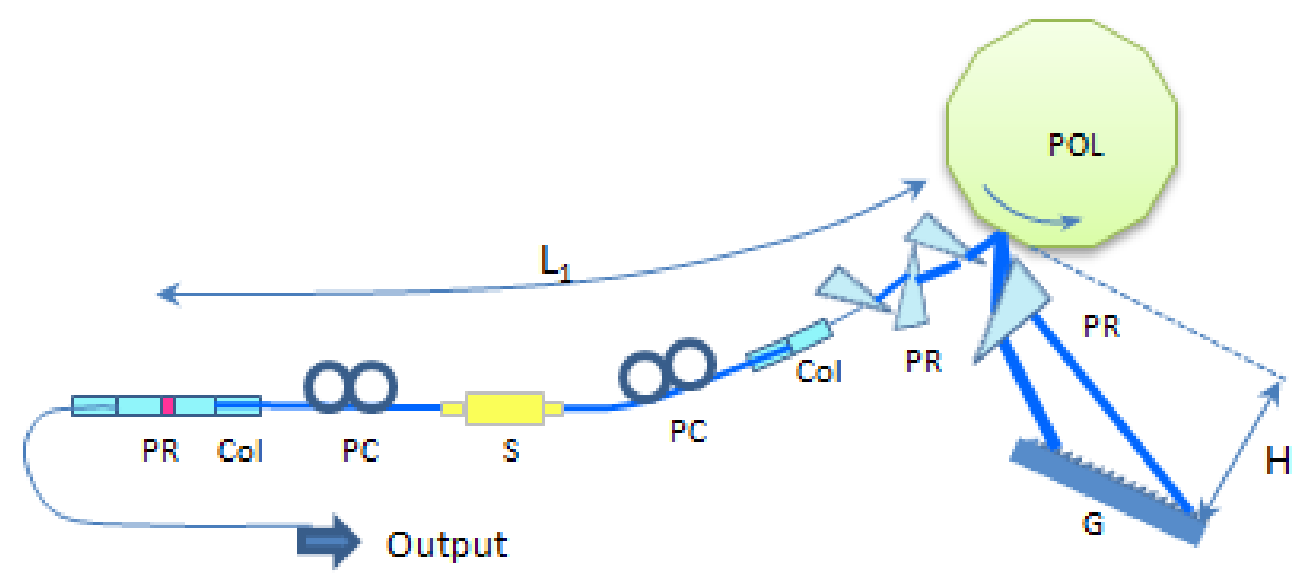

Figure 3.7 Diagram of polygon scanner -based swept laser source PR: Partial reflector, Col: Collimator lens, PC: Polarization controller, S: SOA, PR: Prism, G: Grating, POL: Polygon scanner

\subsection{Results}

Instantaneous linewidth of the source with QPCT was measured at various conditions. The bandwidth (FWHM) of the grating filter of $0.126 \mathrm{~nm}$ and $0.05 \mathrm{~nm}$ was chosen for validation of spectral narrowing effect by QPCT at two different speeds, $2.5 \mathrm{kHz}$ and $20 \mathrm{kHz}$. Filter bandwidth is adjusted by the number of prism expanders inserted in the free-space, thus changing the magnification of the beam. Figure 3.8 shows the oscilloscope trace of the three consecutive scans. Scan range measured by optical spectral analyzer was $55 \mathrm{~nm}$, from 1271 $\mathrm{nm}$ to $1326 \mathrm{~nm}$. Peak and average power are $12 \mathrm{~mW}$ and $8 \mathrm{~m} \mathrm{~W}$, respectively. Relative intensity noise (RIN) of the source was measured less than $-110 \mathrm{~dB} / \mathrm{Hz}$ over all frequency range as shown in Fig. 3.9. The cavity modes spacing of 120-130 MHz, or $0.68 \mathrm{pm}$ to 0.74 $\mathrm{pm}$ in wavelength, accounted for the peak at 120-130 MHz that is inverse of the cavity length, and the splitting of which is due to the modulation of cavity length.

In order to measure the instantaneous linewidth, the swept output is fed into a simple Mach-Zehnder interferometer as shown in Fig. 3.10, and a high-speed receiver with $2.5 \mathrm{GHz}$ bandwidth is used to detect the fringe signal. When the amplitude of fringe drops in half compared to that at zero delay, the amount of delay equals to the coherence length by definition. Relation of instantaneous linewidth and coherence length is given by Eq. (3.7). If 
the dual side of the range around zero delay is included, the range doubles as long as mirror imaging can be cancelled [56-59].

$$
L c=\frac{2 \ln 2}{\pi} \frac{c}{\delta v}
$$

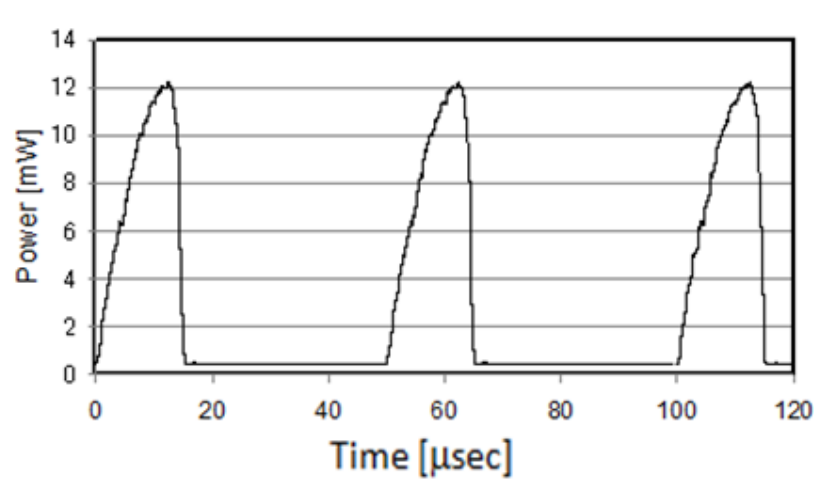

Figure 3.8. Temporal optical output power

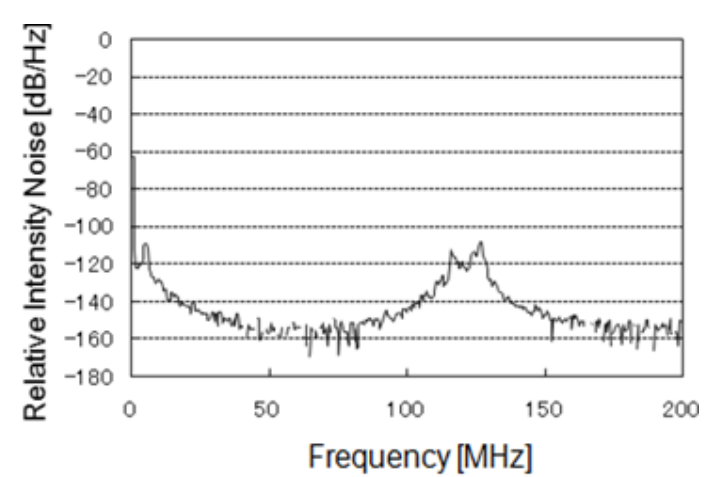

Figure 3.9. Relative Intensity Noise

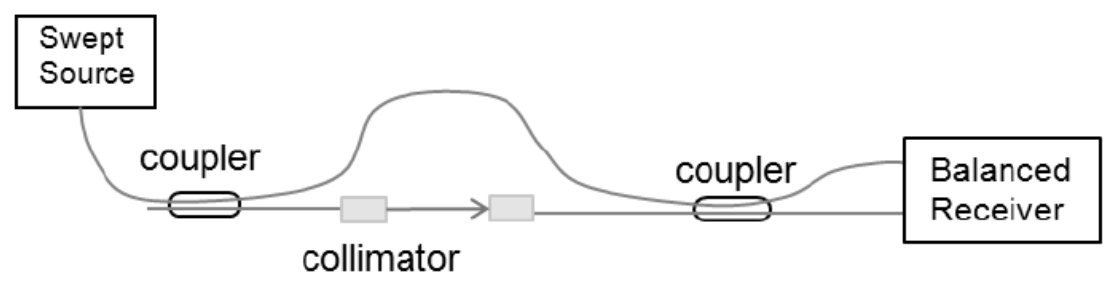

Figure 3.10. Balanced Mach-Zehnder interferometer

Figure 3.11 shows the signal power spectrum at several depth positions, with and without QPCT. The one without QPCT has a ratio of $\mathrm{H} / \mathrm{L}_{1}$ about less than $0.05\left(\mathrm{H}=60 \mathrm{~mm}, \mathrm{~L}_{1}=1.15 \mathrm{~m}\right.$, while the source with QPCT has a ratio of $0.25\left(\mathrm{H}=210 \mathrm{~mm}, \mathrm{~L}_{1}=0.85 \mathrm{~m}\right)$. Here we kept the same cavity length for both cases, about 2.5 meters round trip, at the center wavelength. As shown in Fig. 3.11(a), signal power drops $6 \mathrm{~dB}$ at the depth of about 2 to $2.5 \mathrm{~mm}$ if it is without this technique. The corresponding linewidth nearly equals to the filter bandwidth of $0.13 \mathrm{~nm}$. While, it is extended to over $5 \mathrm{~mm}$ if this quasi-phase continuous condition is satisfied, and which corresponds to $0.075 \mathrm{~nm}$ of spectral linewidth. If we slow down the swept rate, we get further extension of single side depth up to around $8 \mathrm{~mm}$ at $2.5 \mathrm{kHz}$ as shown in Fig.3.12. Axial resolution at $2 \mathrm{~mm}$ depth as shown in Fig. 3.11(b) is found about $24 \mu \mathrm{m}$, which corresponds to about FWHM of $55 \mathrm{~nm}$ of swept range. The obtained swept range was such narrower than FSR of tunable filter $180 \mathrm{~nm}$ (duty ratio of about $30 \%$ ) mainly because the length of grating we used is not long enough to cover whole deflection angle of the beam. We believe that by using longer grating in size or shortening the total cavity length can improve the swept range over the gain bandwidth of SOA. Figure 3.13 shows the comparison of spectral linewidth with and without QPCT over different swept rates. 
Coherence length improvement or linewidth narrowing effect was in the factor of about X1.8 to $\mathrm{X} 2$ from the case without using QPCT over 1-30 kHz swept rate.

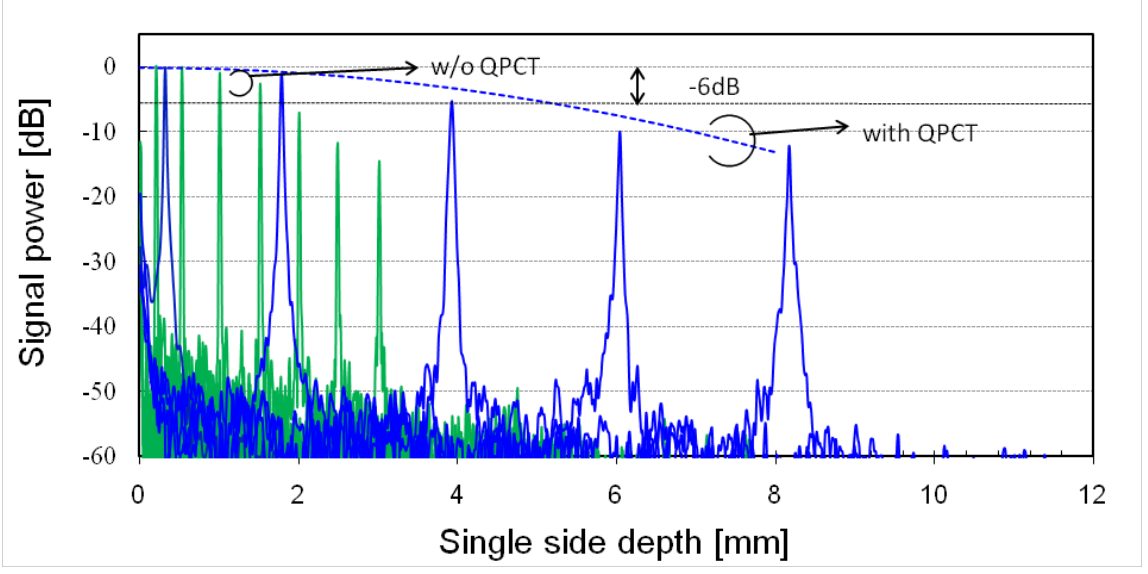

(a)

(b)

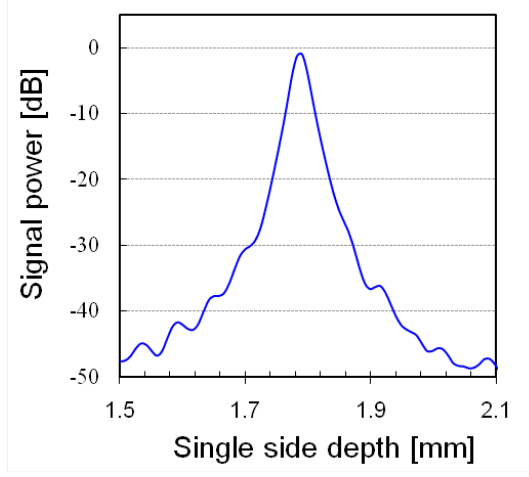

Figure 3.11.(a) OCT signal at different depth positions with and without QPCT condition

(b) Close-up of spectrum $(20 \mathrm{kHz}$ swept rate)

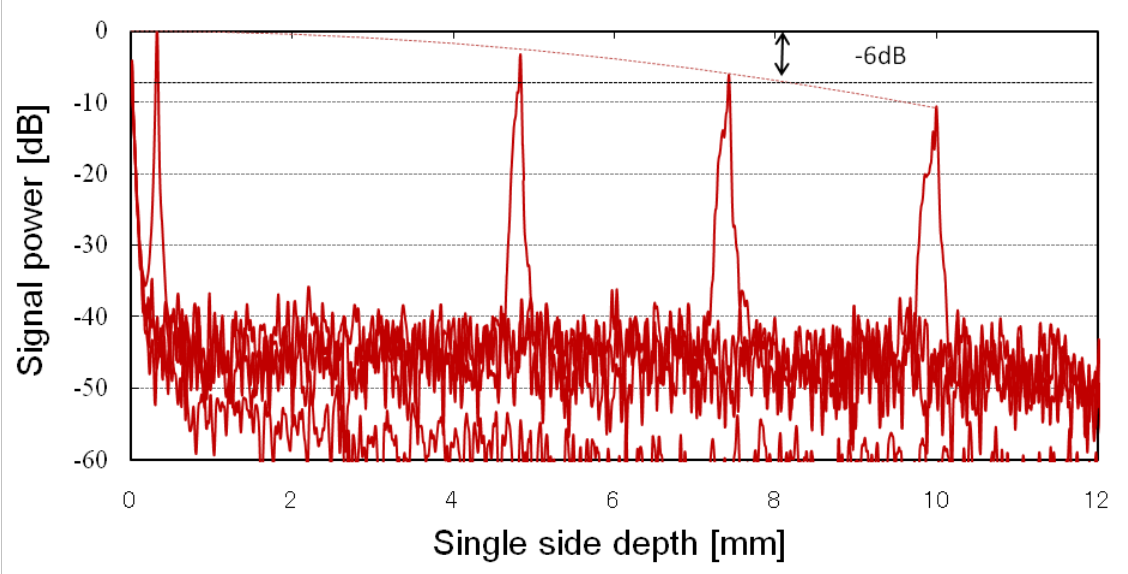

Figure 3.12. OCT signal at different depth positions with and without OPCT condition $(2.5 \mathrm{kHz}$ swent rate) 


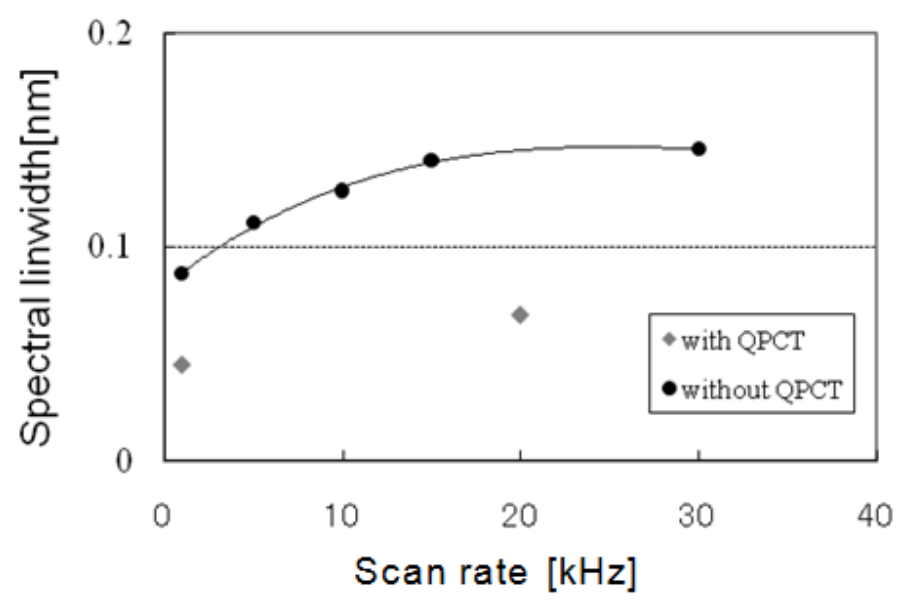

Figure 3.13. Spectral linewidth vs. Scan rate

Replacing the grating to the one with higher modulation constant of $1500 \mathrm{~g} / \mathrm{mm}$ resulted in a further increase of the coherence length. The calculated filter width in the same configuration except the grating was $0.051 \mathrm{~nm}$. Figure 3.14 shows the measured OCT signal spectrum at different positions at two swept rates, $2.5,20 \mathrm{kHz}$. Coherence length was $30 \mathrm{~mm}$, and $17 \mathrm{~mm}$, corresponding to $0.025 \mathrm{~nm}, 0.043 \mathrm{~nm}$ of linewidth respectively at center wavelength of $1289 \mathrm{~nm}$. It was a factor of X1.2 to X2.0 improvement or narrowing with respect to the filter bandwidth depending on the swept rate. Improvement factor becomes lower than the previous case. This is because the phase lag becomes larger when using modulation constant of $1500 \mathrm{~g} / \mathrm{mm}$ than the one with $1312 \mathrm{~g} / \mathrm{mm}$ and also because the phase shift per round trip becomes non-negligible order with respect to the order of linewidth. Table 3.1.summarizes the results with different setting of parameters.

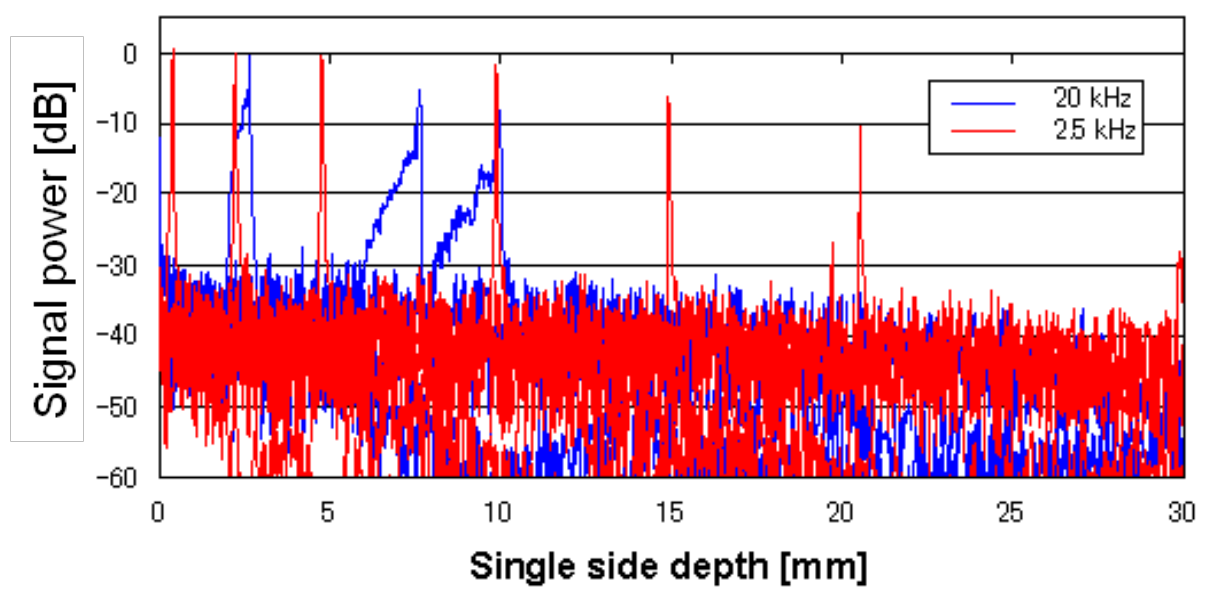

Figure 3.14. OCT signal at different depth positions with and without QPCT condition 
Table 3.1. List of summary results

\begin{tabular}{|c|c|c|c|c|c|c|}
\hline Parameter & \multicolumn{2}{|c|}{ Without QPCT } & \multicolumn{2}{c|}{ With QPCT (1) } & \multicolumn{2}{c|}{ With QPCT (2) } \\
\hline Scan rate & $20 \mathrm{kHz}$ & $2.5 \mathrm{kHz}$ & $20 \mathrm{kHz}$ & $2.5 \mathrm{kHz}$ & $20 \mathrm{kHz}$ & $2.5 \mathrm{kHz}$ \\
\hline Center wavelength & \multicolumn{2}{|c|}{$1310 \mathrm{~nm}$} & \multicolumn{2}{|c|}{$1310 \mathrm{~nm}$} & \multicolumn{2}{|c|}{$1290 \mathrm{~nm}$} \\
\hline Filter bandwidth & \multicolumn{2}{|c|}{$0.126 \mathrm{~nm}$} & \multicolumn{2}{|c|}{$0.126 \mathrm{~nm}$} & \multicolumn{2}{c|}{$0.05 \mathrm{~nm}$} \\
\hline Instantaneous linewidth & $0.149 \mathrm{~nm}$ & $0.09 \mathrm{~nm}$ & $0.075 \mathrm{~nm}$ & $0.048 \mathrm{~nm}$ & $0.043 \mathrm{~nm}$ & $0.025 \mathrm{~nm}$ \\
\hline Coherence length & $5.1 \mathrm{~mm}$ & $8.4 \mathrm{~mm}$ & $10 \mathrm{~mm}$ & $16 \mathrm{~mm}$ & $17 \mathrm{~mm}$ & $30 \mathrm{~mm}$ \\
\hline $\begin{array}{c}\text { Narrowing factor from } \\
\text { filter bandwidth }\end{array}$ & $\mathrm{X} 0.85$ & $\mathrm{X} 1.4$ & $\mathrm{X} 1.7$ & $\mathrm{X} 2.1$ & $\mathrm{X} 1.2$ & $\mathrm{X} 2.0$ \\
\hline
\end{tabular}




\subsection{Intraocular distance measurement}

\subsubsection{Introduction}

Most standard treatment for Cataract is the implantation of intraocular lens (IOL). Prior to the surgery, IOL power should be obtained by measuring the axial length of the eye that is a distance from retina to cornea. The accuracy of measurement is important for conducting precise implantation of IOL. This ranges from $25 \mathrm{~mm}$ to $35 \mathrm{~mm}$ in normal human eye. Conventionally, it was measured by Ultrasonography. Measurement accuracy in this case is limited to 0.05 to $0.1 \mathrm{~mm}$ range, and measurement itself is an invasive measurement in the sense that the measurement probe should be in contact with the cornea through matching gel.
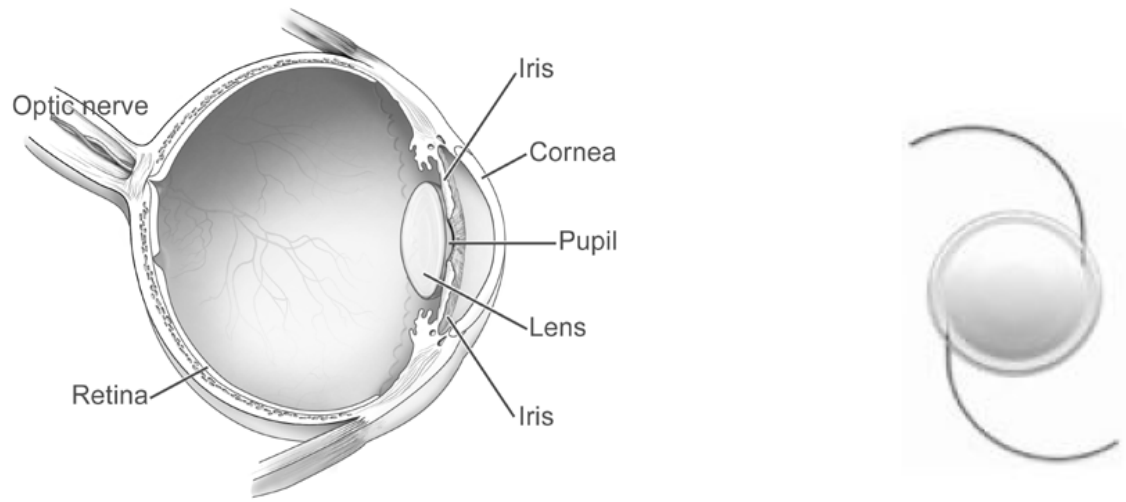

Figure 3.15 Schematic cross-section of a healthy human eye (left: courtesy of the National Eye

Institute, National Institute of Health), and intraocular lens (right)

On the other hand, recently introduced optical methods such as time-domain optical coherence reflectometry [15] improve the accuracy, and which is a non-invasive method by its principle. However, because the measurement time is relatively slow due to slow scanning of mechanical delay line, it the movement of the eye during the measurement may degrade the measurement accuracy. On the other hand, Fourier domain methods such as the OCT for the retinal imaging are known as a popular technique for making high-resolution, high sensitivity imaging possible. Recent work [73] based on Fourier Domain low coherence interferometry similar to SD-OCT at $840 \mathrm{~nm}$ implements two separate reference arms with different optical length so that you can image two depth ranges simultaneously and calculate the axial length just by adding the offset length between two arms. This approach countermeasures the shortcoming of shallow depth range of spectral domain based measurement that is intrinsically restricted by the pixel resolution of spectrometer. However, this approach still forces the user to search an approximate position to find the signal within the measurable range. This will necessitate the mechanical scanning of the 
delay line and also the processing to distinguish the overlapped signals from anterior and posterior area.

Recently, $1060 \mathrm{~nm}$ range wavelength that has gained good attentions for the retinal OCT imaging because of larger penetration compared to that at $840 \mathrm{~nm}$ range [74]. This range combined with Fourier domain methods, either in spectral domain, or in swept source OCT promises a next generation OCT system for the retinal imaging. Our further expectation is that OCT can be extended to the large geometry measurement such as for measuring axial length for IOL calculation. However, in a similar way as explained for the SD-OCT, the most of previously proposed swept sources for OCT have the depth range defined by coherence length ranging around $2 \mathrm{~mm}$, and which is far below the measurement range required for the axial length of the eye, or swept rates of tunable lasers were too slow [75].. In this report, we propose a swept source that implements quasi-phase continuous tuning (QPCT) [76] and multiple beam expanders in order to improve the coherence length up to $28 \mathrm{~mm}$ range at swept rate of $2.5 \mathrm{kHz}$, which is about 5 to 10 times larger than what can be achieved in present systems [77,78]. This swept source enables us a very simple operation of the measurement of the axial length of eye where you only need to divide the obtained distance by the known refractive index. We demonstrate the actual OCT measurement of axial length with using a pig eye. We have confirmed the positions and image of retinal and cornea in $1 \mathrm{D}$ and $2 \mathrm{D}$ OCT images, and the axial length was measured about $27 \mathrm{~mm}$ in optical length.

\subsubsection{Principle and design}

Figure 3.16 shows the actual configuration of the wavelength-swept laser source demonstrated in this study. A fiber pigtailed SOA (COVEGA: BOA1142) and a polygon scanner-based diffraction grating filter are connected together to form an extended ring cavity laser. Effective cavity length is 2.6 meter in round trip. The filter bandwidth is set to about $0.024 \mathrm{~nm}$ by using six prism expanders in free-space and a diffraction grating with 1714 lines/mm modulation constant. Output power is extracted from the fiber coupler with $30 \%$ coupling ratio. Polygon mirror has 30 facets, $60 \mathrm{~mm}$ diameter, and rotates at a speed of $5,000 \mathrm{rpm}$ corresponding to $2.5 \mathrm{kHz}$ swept rate. Repetitive deflection toward the diffraction grating in a Littrow arrangement enables the stable sweep of wavelength and the modulation of large optical delay at the same time. The condition of QPCT i.e. virtually equal gradients of wavelength (i.e. $d \lambda g / d \theta$ ) and phase variation (i.e. $d \lambda L / d \theta$ ) over the tuning range was realized by having the distance between the deflection point and the diffraction grating set in a certain specific ratio with respect to the total cavity length. This condition is same as in 
section 3.3. With given geometry and parameters above, $\mathrm{H}$ and $\mathrm{L}_{1}$ yield $12 \mathrm{~cm}$ and $1 \mathrm{~m}$, respectively for the total cavity length of $2.6 \mathrm{~m}$ round trip in optical length.

As seen in the Fig. 3.17, the phase is not perfectly synchronized over whole tuning range between tunable filter and cavity modes. Figure 3.18 shows the degree of phase synchronization in terms of temporal phase lag between longitudinal modes and the filter's peak wavelength over entire wavelength range as the difference of gradients; $(d \lambda g-d \lambda L) / d \theta$. There are maximum $40 \%$ of phase shift with respect to the tuning wavelength over $40 \mathrm{~nm}$ of tuning range. Conversely, about at least $60 \%$ of cavity longitudinal modes remain in-phase during the sweep over small increment of time, or in the other words, the phase shift per round trip is reduced to $40 \%$ compared to the case of non-QPCT conditions, and which is expected to result in the enhancement of cavity Q factor, or large coherent build-up of gain per round trips.

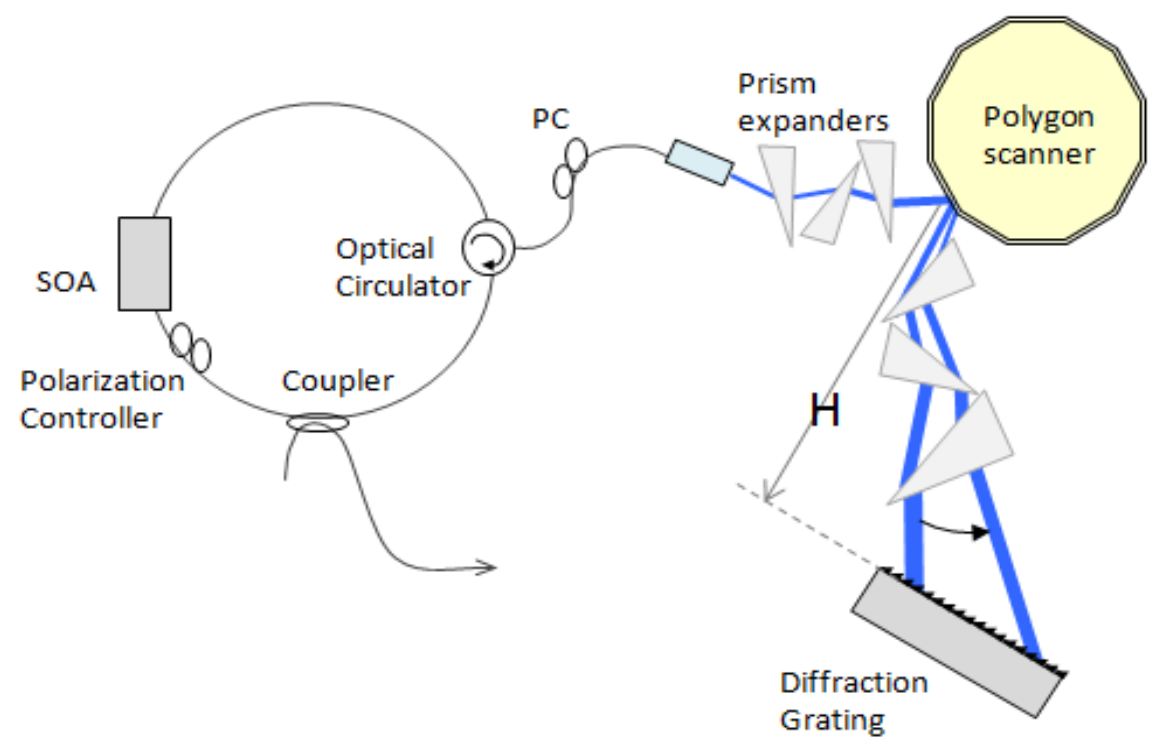

Figure 3.16 Laser configuration

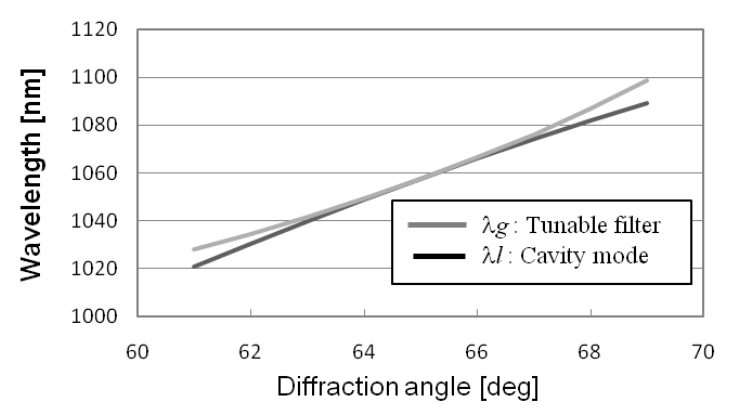

Figure 3.17 Phase change over the range

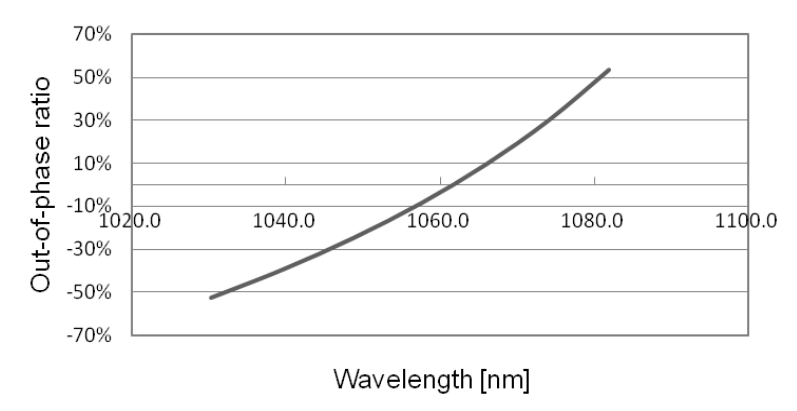

Figure 3.18 Phase shift ratio over wavelegth range 


\subsubsection{Design trade-off}

In the followings, the limitation of the performance is explained. What limits the performance by the geometry is the polygon scanner's rotational speed and diameter, D.

In general, polygon scanner's speed can go up to around 45,000 rpm with sustainable durability and stability with a maximum of 60 to $80 \mathrm{~mm}$ diameter of the mirror in a higher grade when using the one with air-suspended axis. While, the lower grade scanner with slower speed and shorter diameter allows us to use those off-the-shelf, low-cost polygon scanners from the mass produced industrial product lines like the ones for laser printers.

Swept rate $\mathrm{f}$ is the product of rotation rate of the polygon $f_{p}$ and the number of facets $N, f=$ $f_{p} N$. The filter width is defined by the Eq. (3.8), where $w_{i}$ is incoming beam width on the facet of the polygon from the collimator with some magnification prior to the input to the polygon. Effective deflection angle accounting for beam clipping is $\Delta \theta \mathrm{e}=4 \pi\left(1-w_{i} / W\right) / M N$ where facet width $W=\pi D / N$. This is related to the FSR of tunable filter, $\Delta \lambda$ as given in Eq. (3.9). $M$ is the total beam magnification by cascaded prisms by $p$ after the polygon deflection, $M=m p$ where individual magnification of each prism expander, m ranges about $\mathrm{X} 1.6$ if the angle with respect to the normal is set around 55 degree.

$$
\begin{gathered}
\delta \lambda=\frac{2 \sqrt{2 \ln 2} \lambda a \cos \theta_{0}}{\pi M w_{i}} \\
\Delta \lambda=4 a \cos \theta_{0} \cdot \Delta \theta_{e}=\frac{8 \pi a\left(1-w_{i} / W\right) \cos \theta_{0}}{M N} \\
M=m^{p} \\
F=\frac{2 \pi^{2} w_{i}}{\sqrt{\ln 2} N \lambda_{o}}\left(1-\frac{w_{i}}{W}\right)
\end{gathered}
$$

In order to achieve very large coherence length thus very narrow linewidth, original filter bandwidth $\delta v$ before QPCT enhancement should also be narrower. However, when we narrow $\delta v$ by expanding the beam width $w_{i}$, or by reducing grating pitch a, it will also narrow the swept range, $\Delta \lambda$ because effective deflection angle is decreased and the beam clipping will be larger. Finesse is constant as long as $w_{i}$ and $N$ are fixed as expressed in Eq. (3.11), in this case which is calculated to about 1140 when accounting for beam clipping by the polygon mirror when $w_{i}=2.7 \mathrm{~mm}$. Figure 3.19 shows the relation of FSR and filter bandwidth. Finesse of the filter is counter-proportional to the facet number, and which is reduced when the system requires a faster swept rate with a limited rotational speed of polygon. For the axial length measurement as opposed to the $2 \mathrm{D}$ or $3 \mathrm{D}$ OCT imaging, the 
swept rate of a few $\mathrm{kHz}$ is sufficient for the one dimensional measurement of the axial length.

\subsubsection{Laser performance}

Figure 3.20 shows the temporal power profile of the swept source measured by a detector and an oscilloscope. Output power was $6.2 \mathrm{~mW}$ at peak, and $3 \mathrm{~mW}$ in average. Swept wavelength range was $18 \mathrm{~nm}$ around $1064 \mathrm{~nm}$ with $40 \%$ duty ratio. Coherence length measured by simple Mach-Zehnder interferometer was $28 \mathrm{~mm}$ corresponding to $0.018 \mathrm{~nm}$ in instantaneous linewidth at $2.5 \mathrm{kHz}$ swept rate. Instantaneous linewidth is narrowed by a factor of X1.37 from the filter bandwidth of $0.024 \mathrm{~nm}$ corresponding to the coherence length of $20.4 \mathrm{~mm}$. We also measured the coherence length in the case that QPCT is not used by having geometry not meeting QPCT condition but keeping the same cavity length. Without QPCT, instantaneous linewidth was equal to the filter bandwidth. QPCT with factor of X1.37 narrowing is not as effective as in the experiment with a similar configuration using $1.3 \mu \mathrm{m}$ range SOA that we have done in section 3.5. We confirmed a factor of X2 improvement from the filter bandwidth of $0.05 \mathrm{~nm}$ to instantaneous linewidth of $0.025 \mathrm{~nm}$ to achieve a comparable coherence length of $30 \mathrm{~mm}$ in $1.3 \mu \mathrm{m}$ using a grating with $1500 \mathrm{~g} / \mathrm{mm}$ constant and a single prism expander. This difference in the results in terms of improvement factor can be accounted for by the difference in the cavity gain and loss factor attributed to the performance of SOA and the loss of tunable filter for $1.06 \mu \mathrm{m}$ and $1.3 \mu \mathrm{m}$. Net gain, i.e. the sum of lower gain of $1.06 \mu \mathrm{m}$ SOA compared to that of $1.3 \mu \mathrm{m}$ and larger loss of circulator in the filter for $1.06 \mu \mathrm{m}$, as well as lower ASE power of SOA enforces more round trips until the gain gets accumulated to the saturated regime than in the case of $1.3 \mu \mathrm{m}$, resulting in a larger phase shift between tunable filer and lasing wavelength. It is also partially because the degree of phase synchronization is not sufficiently high when using a grating with modulation constant as high as $1714 \mathrm{~g} / \mathrm{mm}$ in this case.

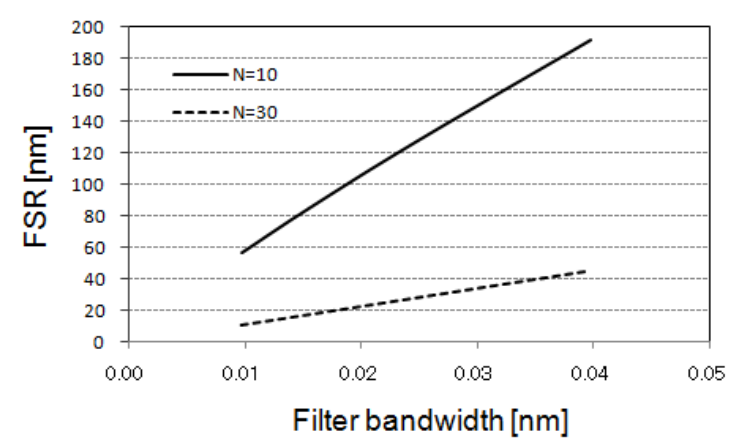

Figure 3.19 Filter bandwidth vs. FSR of tunable filter

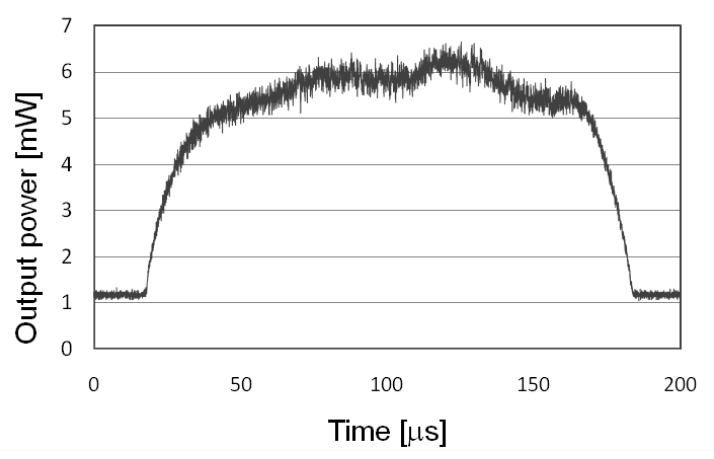

Figure 3.20 Temporal profile of output power 
However, factor of X1.37 improvement provides about $8 \mathrm{~mm}$ increase in coherence length in this case compared to the coherence length without using this technique, which is still a significant improvement of measurement range.

Difference in the measured swept range, $18 \mathrm{~nm}$, and theoretical value, $28 \mathrm{~nm}$ according to the Eq. (3.9) is due to the additional beam cut-off by the edges of prisms and diffraction grating. The use of optimized polygon scanner with a smaller number of facets as well as prisms and a diffraction grating with larger size will increase the swept range further up to over $80 \mathrm{~nm}$, maintaining the same coherence length. As in Fig.3.19, a polygon mirror with ten facets should give over $80 \mathrm{~nm}$ of FSR while maintaining several $\mathrm{kHz}$ swept rate. So lasing with a finesse of over 2,000 is possible in the future by optimizing the polygon mirror and other optics.

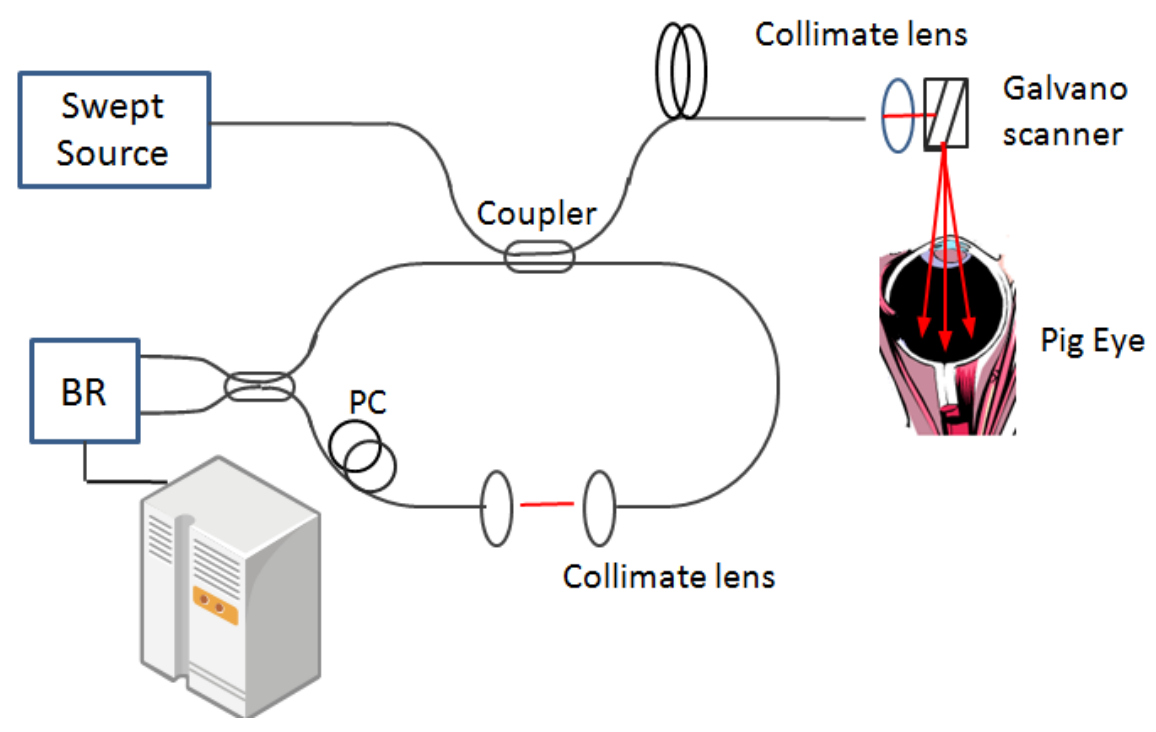

Figure 3.21 OCT system setup for IOL measurement

\subsubsection{Axial length measurement}

The OCT system for the measurement comprises a circulator-less, unbalanced interferometer as shown in the Fig. 3.21, which doesn't use optical circulator to efficiently circulate the signal back to the receiver. We can compromise the sensitivity to a certain degree because we only want to measure a distance of two points. The other process step is similar to the previous work [41]. Optical output from the source is introduced into a fiber-based Mach-Zehnder interferometer with a 70:30 2x2 fiber coupler, and a 50:50 2x2 
fiber coupler. The $30 \%$ reference port is connected through the collimator link to the second coupler. The through port with $70 \%$ coupling ratio of the first coupler is connected to a probe arm and the detected light goes backward to meet at the second coupler. Probe arm comprises a collimator lens with a beam width of $0.8 \mathrm{~mm}\left(1 / \mathrm{e}^{2} \mathrm{FWHM}\right)$ and a Galvano scanner (Cambridge Technology 6210H). We didn't use an objective lens after the Galvano scanner to form a telecentric scan, instead we scanned the beam as coming from the collimator in order to gain a long working distance in terms of focusing depth. Lateral resolution of $0.8 \mathrm{~mm}$ is not important because the scanning is just to give a range and geometry of imaging area to be captured. Distance between two collimator links is adjusted so that the zero delay matches the focus area of the probe arm. Here, optical lengths in the fiber and in the free space are carefully trimmed and spliced so that ratio of those two almost matches between both paths in order to suppress the wavelength dispersion due to the interferometer itself. Probe and reference beams are recoupled by the second coupler and two outputs are fed into the balanced photoreceiver (newfocus 1544). Pig-eye was used as a sample in this study. The spectral interference signal is converted to voltage, and digitized by a 14-bit DAQ board (PDA14, Signatec.Inc.) with sampling rate of $100 \mathrm{Msample/sec} \mathrm{that}$ is mounted inside a PC unit. The start trigger for A-scan synchronization is fed from the integrated start trigger output from the source into the A/D converter. Each start trigger initiates the segmented sampling of about 40000 counts that are transported to the PC, followed by FFT calculation. In the FFT calculation, wavelength-rescaling process in $\mathrm{k}$-space is performed by using non-linear calibration data taken at about $12 \mathrm{~mm}$ position with a simple reflector. Probe power was kept below about $1.5 \mathrm{~mW}$ in average.

OCT signals after the above rescaling process with $35 \mathrm{~dB}$ anti-reflection plate at different depths (single pass) are shown in the Fig. 3.22. Sensitivity is over $95 \mathrm{~dB}$ near zero delay. Signal drops $6 \mathrm{~dB}$ about at the $14 \mathrm{~mm}$ depth corresponding to the coherence length of $28 \mathrm{~mm}$, and the signal can still be recognized even at the depth of over $30 \mathrm{~mm}$

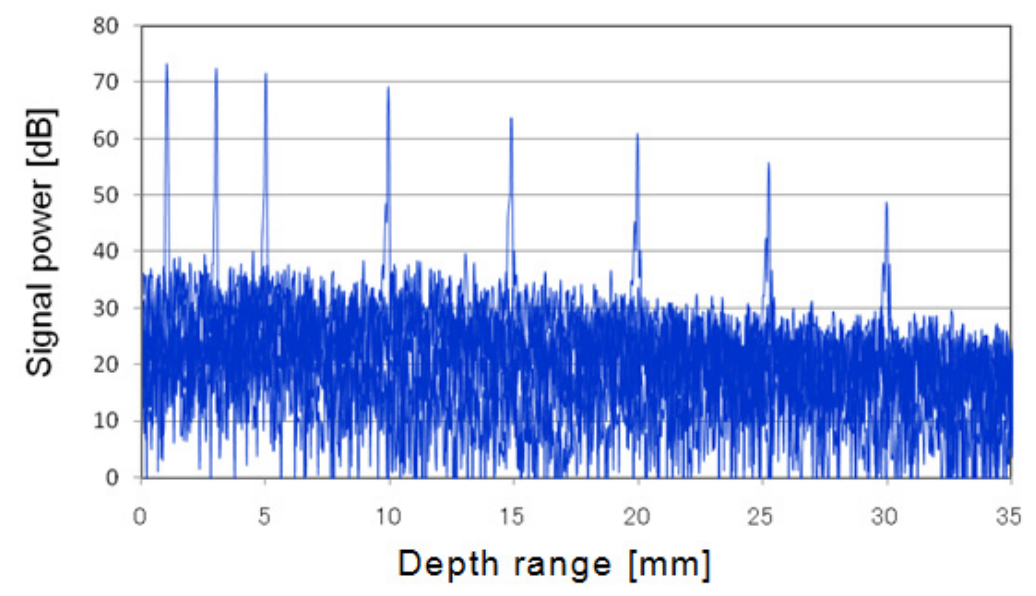

Figure 3.22. Signal power at different depth 
3.6.5 Results

Figure 3.23(a) shows the tomograph image of whole eye over $5 \mathrm{~mm}$ width. The image that we constructed has 200 A-lines in transverse direction over $5 \mathrm{~mm}$. So, pixel resolution is simply $25 \mu \mathrm{m}$. Contour of cornea and iris, lens surface and the retina at far side are all recognized, although the detail of each segment is slightly blurred because lateral resolution is only $0.8 \mathrm{~mm}$. Figure $3.23(\mathrm{~b})$ shows 1-dimensional signal indicating the positions of different parts. Peaks at cornea, lens and retina are apparent and the distances of each other are $3.5 \mathrm{~mm}$ and $27 \mathrm{~mm}$ between cornea and lens, lens and retina, respectively. Assuming the refractive index of 1.35 , physical length is calculated to be $20 \mathrm{~mm}$ as axial length.

The distant side of posterior segment experiences larger loss than anterior due to absorption and scattering through a large distance for the light to travel. This results in additional signal drop on top of that due to a finite coherence length. W Repeatability of measurement was less than $20 \mu \mathrm{m}$ that is better than the performance of commercial IOL measurement equipment (e.g. IOL Master, Zeiss Meditec)
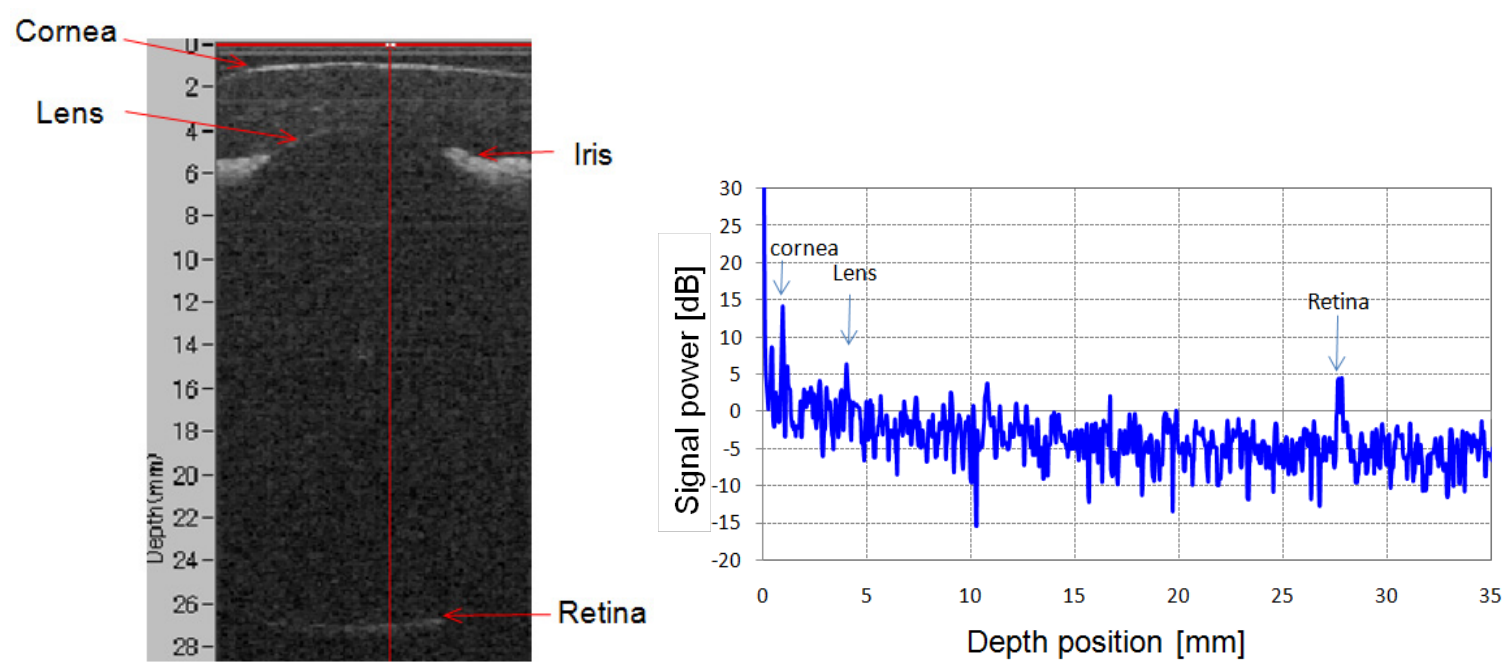

(a) 2-dimentional image of whole eye

(b) 1-dimentional signal of one A-line(red solid line in (a)

Figure 3.23 IOL measurement of the pig-eye 
Results with human eye

Fig.3.24 (a) shows tomography of whole eye (volunteered healthy Asian male, with dioptor -7.5) within a window of $5 \mathrm{~mm}$ width. Probe power was below $1 \mathrm{~mW}$ for eye-safety. Structure of anterior segment including cornea, iris, and lens surface is clear, retina is found at a distance of $39 \mathrm{~mm}$ which can be converted to physical size of $28.9 \mathrm{~mm}$ using effective refractive index of 1.35 . The distant side of posterior segment experiences larger loss than anterior due to absorption through a large distance for the light to travel. This results in additional signal drop on top of that due to a finite coherence length. Fig.3.24 (b) shows the image set in negative side around zero delay where the posterior segment is reversed in the depth scale so that it balances the absorption loss and the signal drop due to coherence length maintain the visibility of signal for the retina.

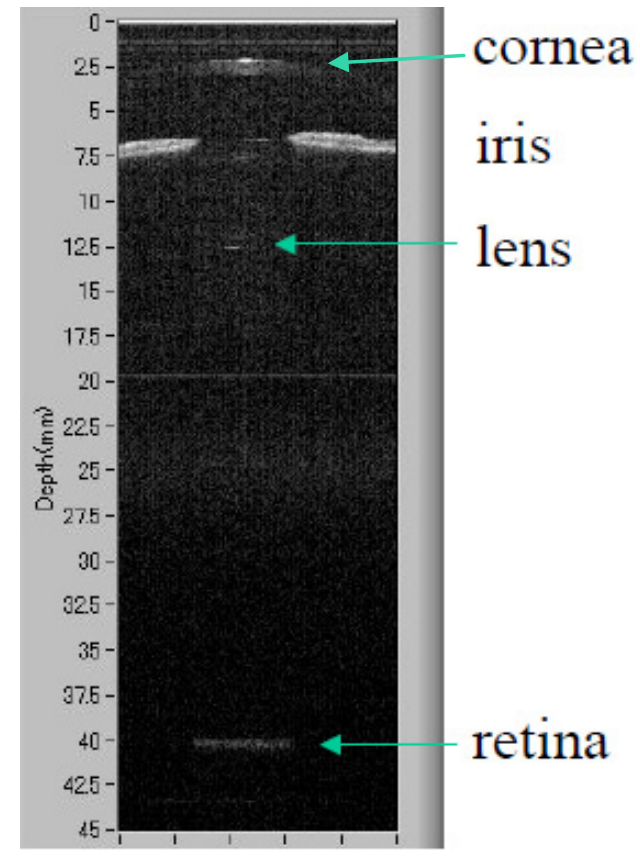

(a) OCT image in positive delay side

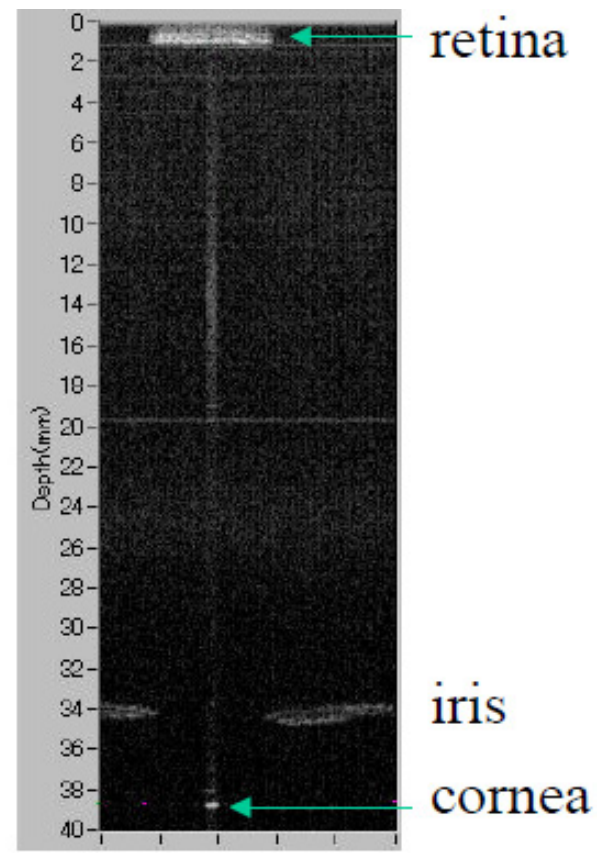

(b) OCT image in negative delay side

Figure 3.24 IOL measurement of human eye 


\subsection{Estimate of spectral width in multimode lasing in quasi-stationary state}

When QPCT is close to the ideal case, i.e. perfect phase matching condition, instantaneous linewidth is expected to be as narrow as the stationary linewidth. So estimating the stationary linewidth will provide the quantitative factor of how much narrowing effect can benefit to instantaneous linewidth during the scan in QPCT. When the lasing is stationary in wavelength, the spectrum can be simply measured by optical spectral analyzer (OSA) unless the linewidth is smaller than resolution of OSA.

Theoretical estimation of spectral width in this type of multimode Gaussian envelope lasing can be derived from the analysis of mode suppression ratio that is calculated by expanding rate equations in multimode oscillation [79],

$$
\begin{gathered}
\frac{d N}{d t}=\frac{\eta_{i} I}{q V}-\frac{N}{\tau}-\sum_{m} v_{g} g_{m} N_{p h m} \\
\frac{d N_{p h m}}{d t}=\Gamma v_{g} g_{m} N_{p h m}+\Gamma \beta_{s p} R_{s p m}-\frac{N_{p h m}}{\tau_{p h}}
\end{gathered}
$$

where variables of $N$ and $N_{p h m}, I$ are total carrier density, photon density on $m$ th cavity mode, and injection current, respectively, and constants of $v_{g}, V, \Gamma, \tau, n_{g}, g_{m}, \beta_{s p}, R_{s p m}$ are group velocity, volume of active region in the gain medium, confinement factor, cavity life time, group index, spontaneous emission factor, spontaneous electron recombination rate, respectively. In the steady state (i.e. $\left.d N / d t=0, d N_{p h} / d t=0\right)$, Eq. (3.12), (3.13) yield the followings; (3.14), (3.15) for photon density and injection current.

$$
\begin{gathered}
N_{p h m}=\frac{\Gamma \beta_{s p} R_{s p m}}{1 / \tau_{p h}-\Gamma v_{g m} g_{m}}=\frac{\Gamma \beta_{s p} R_{s p m} / v_{g m}}{\alpha_{i}+\alpha_{m m}-\Gamma g_{m}} \\
I=I_{t h}+\frac{q V}{\eta_{i}} \sum_{m} v_{g} g_{m} N_{p h m}
\end{gathered}
$$

Here, $I_{t h}, \alpha_{i}, \alpha_{m m}, \eta_{i}$ are threshold injection current, internal optical loss of gain medium, the sum of mirror loss and filter loss at $m$ th mode, internal efficiency, respectively.

Optical power at a cavity mode order (m) can be expressed as in Eq. (3.16).

$$
P_{m}=F_{1 m} v_{g m} \alpha_{m} N_{p h m} h v V_{p}=\frac{\alpha_{m} \Gamma R_{s p m}^{\prime} h v V_{p}}{\alpha_{i}+\alpha_{m m}-\Gamma g_{m}}
$$

Here, $F_{1 m}, \alpha_{m}$, is the ratio of power coupled out, and mirror loss that is virtually equal for all modes in this case because of narrow filter window. Mode suppression ratio (MSR) is 
simply given as the ratio of output power of primary mode $(m)$ to that of the distant $n$th mode $(n)$.

$$
M S R=\frac{P_{n}}{P_{m}}=\frac{g_{t h n}-g_{n}(N)}{g_{t h m}-g_{m}(N)}
$$

If modal gain and threshold gain at each mode are given, MSR can be calculated from Eq. (3.17). Figure 3.25 shows the optical power per mode with a small gain or loss difference. In the case of multimode laser oscillation with narrow Gaussian envelope as depicted in Fig.3.25 (b), modal gain and carrier densities are localized in degenerate states so that the group of modes experiences simply a cavity loss variation enforced by the tunable filter at different mode positions. Figure 3.26 (b) shows the MSR against carrier density per mode. Since carriers are spread to a number of modes within the window, MSR is expected to be substantially lower than that in the case of single mode lasing. Alternatively, total power can be expressed as in Eq. (3.18), and actual suppression ratio can be simply the ratio of the total output power $P_{\text {total }}\left(\alpha_{m m}(\lambda c)\right)$ to the output power with insertion loss equivalent to the filter transmission loss at $n$th mode position, $P_{\text {total }}\left(\alpha_{m n}(\lambda c+\delta \lambda)\right)$.

$$
\begin{gathered}
P_{\text {total }}=\eta_{i} \frac{\alpha_{m}}{\alpha_{i}+\alpha_{m m}} \frac{h v}{q}\left(I-I_{t h}\right)=\frac{\alpha_{m}}{\alpha_{i}+\alpha_{m m}} h v V \sum_{m} v_{g} g_{m} N_{p h m} \\
M S R=\frac{P_{\text {total }}(+\Delta \alpha)}{P_{\text {total }}}=\frac{\left(\alpha_{i}+\alpha_{m m}\right)\left(I-I_{t h n}\right)}{\left(\alpha_{i}+\alpha_{m n}\right)\left(I-I_{t h}\right)}
\end{gathered}
$$

where $I_{t h}, I_{t h n}$ are total threshold current on all modes and threshold current when having the filter loss coinciding on $n$th mode position, respectively. The output power at each mode position is emulated by changing the cavity loss, for an example by adding the attenuation inside the external cavity. $1.5 \mathrm{~dB}$ and $3 \mathrm{~dB}$ neutral density attenuation plate are inserted in the optical path. Figure 3.27 shows the output power against injection current to the SOA with $0,3,6 \mathrm{~dB}$ cavity loss increase. The ratio of output power at two different losses with respect to the $0 \mathrm{~dB}$ loss corresponds to MSR stretched from filter's profile as shown in Fig. $3.26(\mathrm{a})$, which is about $7 \mathrm{~dB}$ and $11 \mathrm{~dB}$ in this example. This gives an approximate profile of the lasing spectrum as shown in Fig. 3.28. Calculated linewidth from the Gaussian fitted profile using the MSR is $0.075 \mathrm{~nm}$, and the stationary linewidth measured with OSA was $0.052 \mathrm{~nm}$ which is close to the instantaneous linewidth at $2.5 \mathrm{kHz}$ swept rate; $0.048 \mathrm{~nm}$. Insufficient data sampling of profile and the error of inserted attenuation value as well as mismatch between actual filter profile and the ideal Gaussian envelope account for discrepancy between the calculated and the measured linewidth. However, it gives an approximate estimate of linewidth especially when the spectral linewidth is smaller than the 
measurable resolution of OSA, which is a convenient way to assess the linewidth limit when using QPCT technique.

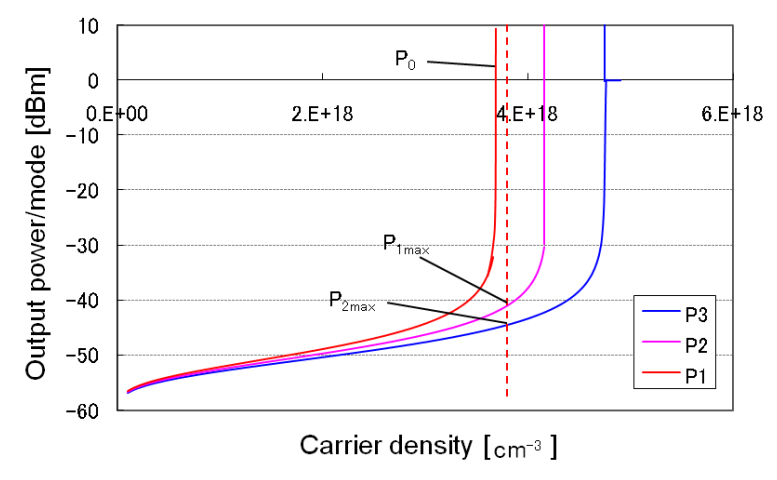

(a) Single mode lasing

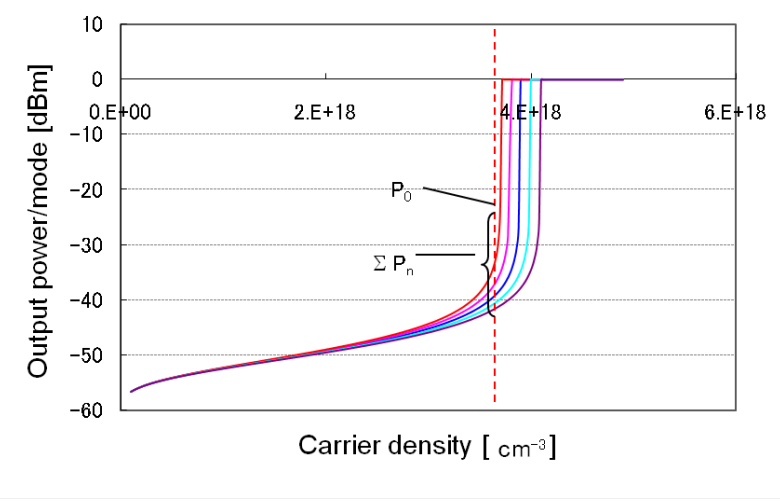

(b) Multi-mode lasing

Figure 3.25 Light vs. carrier density for $m$-th cavity mode

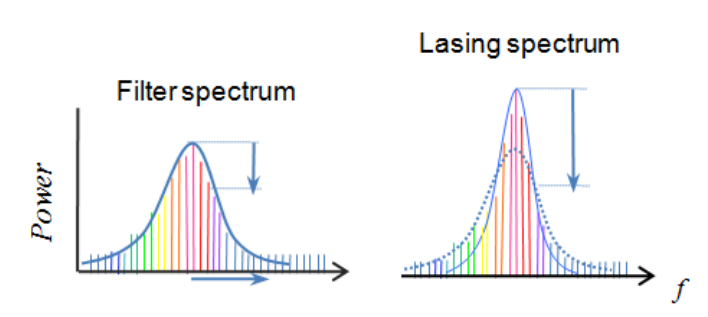

(a)

Figure 3.26 Mode suppression ratio

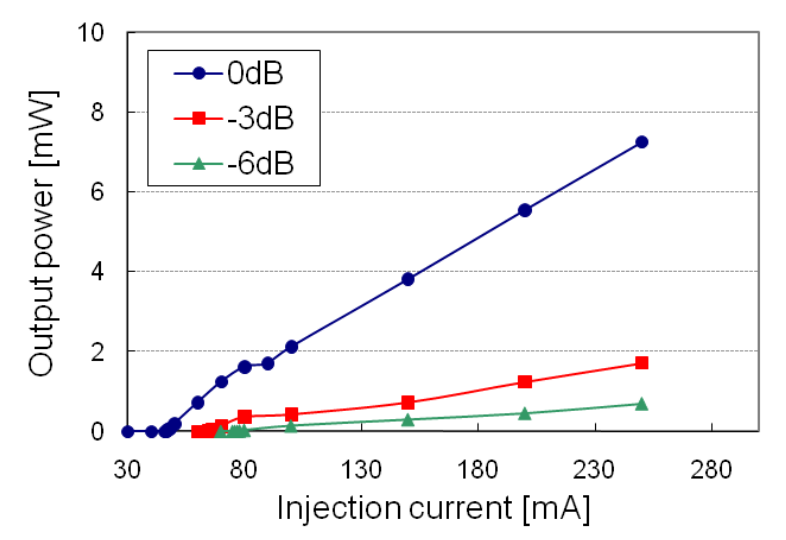

Figure 3.27 Output power vs. Injection current with additional cavity loss

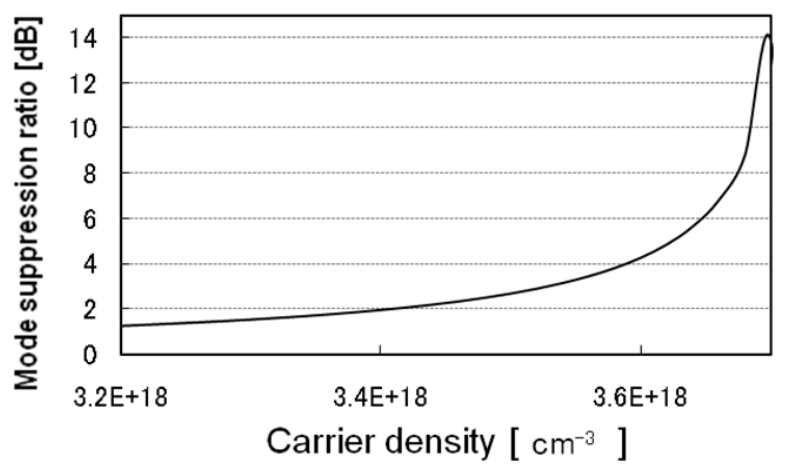

(b)

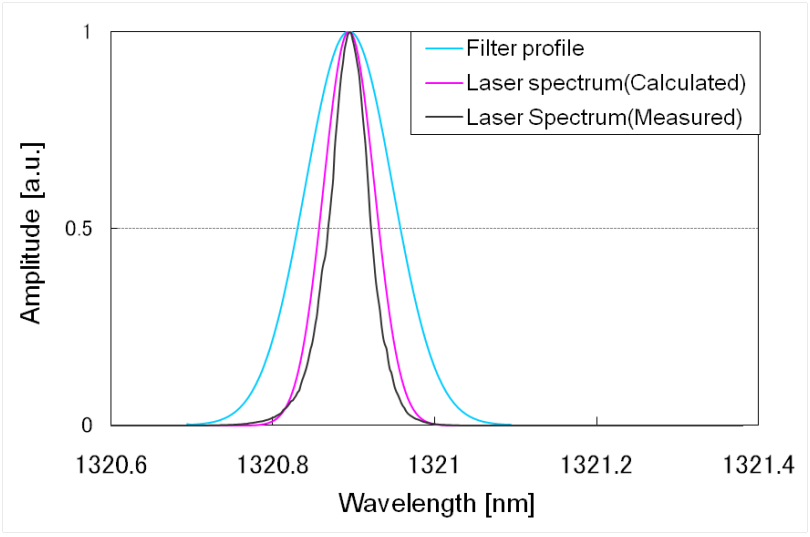

Figure 3.28 Spectrum comparison 
In addition, Eq. (3.18) indicates the relation of the modal density contributing to the laser. If cavity mode density within the laser spectrum window is lower, distributed carrier density per mode becomes higher leading to higher modal gain; $g(N)$, thus resulting in higher MSR as can be seen in Fig. 3.26(b). It suggests that the cavity length should be shorter to give lower cavity density for narrowing the linewidth. Shorter limit for cavity length is restricted by the requirement of continuity of tuning, not inducing bistability of lasing or significant mode hopping. Increase of injection current will also increase the MSR up to the saturated regime, while MSR starts to decrease in the saturated regime when the gain is compressed by excessive depletion of carrier. 


\subsection{Summary}

In this chapter, we proposed and demonstrated a high-speed wavelength swept laser with a technique to improve coherence length drastically by novel quasi-phase continuous tuning (QPCT). QPCT configured in the extended cavity tunable laser in Littrow configuration enhances the instantaneous linewidth while maintaining the continuous wavelength sweep at high swept rate. Using this technique, instantaneous linewidth of $0.048 \mathrm{~nm}$ and $0.075 \mathrm{~nm}$ was realized at sweep speed of $2.5 \mathrm{kHz}, 20 \mathrm{kHz}$, respectively, which corresponds to the coherence length of $16 \mathrm{~mm}, 10 \mathrm{~mm}$ at $1310 \mathrm{~nm}$ wavelength range. Linewidth narrowing factor by QPCT is about X1.7 to X2.0 with respect to the tunable filter's bandwidth depending on the swept rate. We confirmed this approach is non-resonant with respect to the swept rate unlike frequency-shifted wavelength swept source or FDML laser proposed in the previous works. Further optimization with using a shorter cavity design and smaller grating modulation constant will improve the enhancement factor. The QPCT technique will contribute not only to the large-depth imaging in OCT applications but also to the high-resolution spectroscopy or dynamic fiber sensing applications as these are gaining attentions in the near future. 


\section{Chapter 4 Wide range swept-source for high resolution OCT imaging}

\subsection{Objective}

High-resolution imaging is important especially when optical biopsy is necessary such as for cancer diagnosis. Wide tuning range is one of demanding features of the swept source because it improves the axial resolution in the OCT imaging, thus broadening the window of applications for SS-OCT [80]. What limit the tuning range of the swept source are two factors; gain bandwidth of SOA and FSR of tunable filer in the case of extended cavity type laser source. Previous works reported a total tuning range of $133 \mathrm{~nm}$ with a single SOA [53] and $145 \mathrm{~nm}$ with a dual ring configuration with two SOAs [52]. However, such combination of Littman configuration and polygon scanner necessitates the relatively complicated optics in order to minimize the beam walk-off by polygon scanner. In this chapter, we discuss and propose wide range wavelength-swept laser source with a simple configuration using a diffractive grating and a polygon scanner based on Littrow configuration with a single SOA device. The proposed Littrow configuration with a polygon scanner and prisms realizes a simple arrangement leading to good manufacturability while maintaining solid performance. Comparison of Littrow and Littman arrangement is also discussed in terms of efficiency of tuning.

Further wide tuning is proposed by implementing spatial spectral shaping technique that controls the wavelength dependent loss inside the laser cavity so that SOA's gain is efficiently used over the wavelength range. The laser exhibits $20 \mathrm{~mW}$ output power with 170 $\mathrm{nm}$ edge to edge scan range, $160 \mathrm{~nm}$ FWHM at scanning rate of $20 \mathrm{KHz}$. Alternative technique we introduce is the method to modulate the injection current over wavelength range synchronizing the timing with A-trigger from the source.

In the last section, further improvement of sweep speed of $50 \mathrm{kHz}$ is proposed which exceed the Finesse of the swept source that uses telescopic lens system $[51,52,54]$. 


\subsection{Semiconductor Optical Amplifier}

The SOA (COVEGA: BOA1132) has very wide gain bandwidth which is almost equal to the ASE bandwidth which is typically $100 \mathrm{~nm}$ FWHM with $28 \mathrm{~dB}$ gain at $1312 \mathrm{~nm}$ as shown in Fig. 4.1. Swept range is limited by finite gain level at the edges of bandwidth. In normal swept source operation, injection current is set to constant over time, so what limits the maximum swept range is maximum rating current and catastrophic optical damages (COD) on the gain chip facet. Further improvement of intrinsic gain bandwidth requires careful engineering of quantum well structure of the chip.

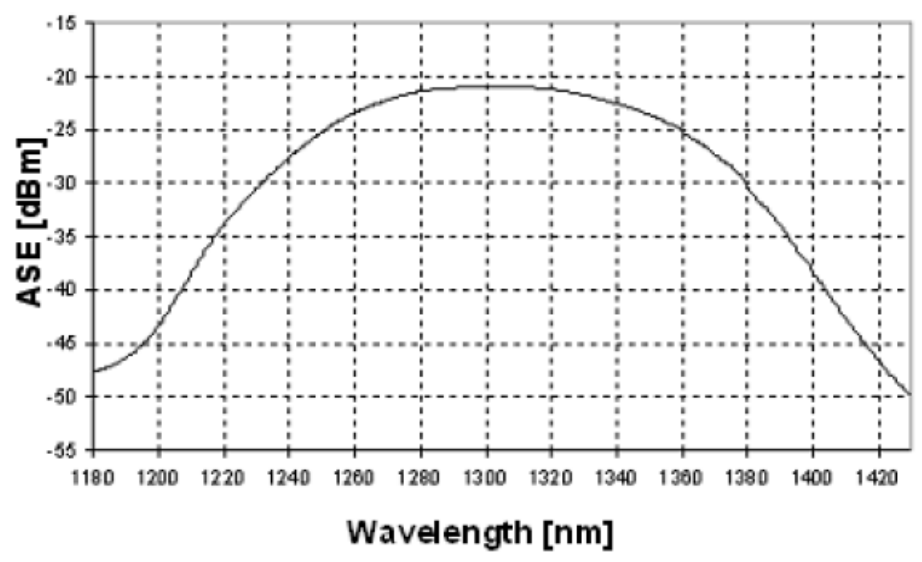

Table 4.1 Typical specification of SOA (COVEGA: BOA1132)

\begin{tabular}{|l|c|c|c|c|c|}
\hline \multicolumn{1}{|c|}{ ITEM } & SYM. & MIN. & TYP. & MAX. & UNIT \\
\hline Operating current & Iop & & 600 & 750 & $\mathrm{~mA}$ \\
\hline Center Wavelength & $\lambda \mathrm{C}$ & 1290 & 1300 & 1315 & $\mathrm{~nm}$ \\
\hline Optical 3dB bandwidth & BW & 70 & 90 & & $\mathrm{~nm}$ \\
\hline Saturation Output Power & Psat & 12 & 15 & & $\mathrm{dBm}$ \\
\hline Small Signal Gain & $\mathrm{G}$ & 17 & 20 & & $\mathrm{~dB}$ \\
\hline Gain Ripple(p-p) & $\delta \mathrm{G}$ & & 0.3 & 0.5 & $\mathrm{~dB}$ \\
\hline Polarization Dependent Gain & $\mathrm{PDG}$ & & & 1 & $\mathrm{~dB}$ \\
\hline Noise Figure & $\mathrm{NF}$ & & 12 & 14 & $\mathrm{~dB}$ \\
\hline Forward Voltage & $\mathrm{Vf}$ & & 1.4 & 2 & $\mathrm{~V}$ \\
\hline & & & & & \\
\hline TEC operation & & & & & \\
\hline TEC current & Itec & & tbs & tbs & $\mathrm{A}$ \\
\hline TEC voltage & Vtec & & tbs & tbs & $\mathrm{V}$ \\
\hline Thermistor Resistance & Rth & & $10 \mathrm{~K}$ & & ohm \\
\hline Integrated isolator & & & & & \\
\hline
\end{tabular}




\subsection{Discussion of tuning range: Comparison with Littman configuration}

This section discusses the comparison of Littrow and Littman configuration, explaining the reason why we have selected the Littrow configuration for the combination with polygon scanner in order to achieve the wide scanning range. First, we compare the efficiency of sweep by quantifying the tuning range at a same scanning rate for Littman and Littrow configuration as shown in Fig. 4.2.

The tuning ranges for Littman and Littrow are expressed in following respectively,

$$
\begin{gathered}
\Delta \lambda_{\text {Littman }}=p \cdot \cos \phi \cdot \Delta \theta_{m} \\
\Delta \lambda_{\text {Littrow }}=4 a \cos \theta_{0} \cdot \Delta \theta_{m} / M
\end{gathered}
$$

Where $\mathrm{p}$ and $a$ are the pitch of diffraction grating for the case of Littman and Littrow respectively, and $\theta_{0}$ is the Littrow angle corresponding to the center wavelength in the range, $\phi$ is the diffraction angle in Littman configuration as shown in Fig.4.2, $M$ is a magnification of the prism expander in the proposed Littrow configuration, $\Delta \theta m$ is the deflection angle by scanning mirror.

While, the filter bandwidth for each case is given by:

$$
\begin{gathered}
\delta v_{\text {Littman }}=\frac{\lambda p \cos \varphi}{2 \pi \omega_{i}} \\
\delta v_{\text {Littrow }}=\frac{\lambda a \cos \theta_{0}}{\pi M \omega_{i}}
\end{gathered}
$$

where, $\varphi$ is the incident angle to the grating in the case of Littman configuration.

For this comparison we consider the case that both configurations have the same filter bandwidth, both have the same input beam width $w i$ as well as same deflection angle of scanner mirror. These condition yields the relation of $p \cos \varphi / 2=a \cos \theta_{0} / M$. Together with this, comparing Eq. (4.3) and (4.4) derives to

$$
\Delta \lambda_{\text {Littrow }}=2 \frac{\cos \varphi}{\cos \phi} \Delta \lambda_{\text {Littman }}
$$

So, for an example, at the glazing incidence, angle $\varphi$ for Littman is normally around $1.2 \mathrm{rad}$ for conventional diffraction grating. If the $p$ is set at $1 / 800 \sim 1 / 1300$ lines $/ \mathrm{mm}$, then $\lambda_{\text {Littman }}=p(\sin \phi+\sin \varphi)$ yields $\phi=0.0 .11 \sim 0.89 \mathrm{rad}$. So, the term $\cos \varphi / \cos \phi$ becomes in the range of $0.7 \sim 1.1$. So the tuning range of Littrow is almost equivalent to that of Littman, 
provided that the linewidth is set to equal in both cases.

Furthermore, when combined with a polygon scanner, Littman configuration suffers beam walk-off issue as depicted in Fig. 4.3 unless the complementary optics such as telescopic lens pair is implemented between a diffraction grating and polygon scanner. On the other hand, since the beam width against the polygon facet is relatively small in the case of Littrow, loss of tuning range by walk-off is practically small so that sufficient duty cycle of lasing can be achieved. Considering these facts, the proposed Littrow configuration with a polygon scanner and prisms simplifies the arrangement leading to better manufacturability, reliability while maintaining equivalent performance to the case using Littman configuration. Efficiency of geometry or design trade-off can be correlated with Finesse, the ratio of tuning range to linewidth at a certain speed, which is introduced as a comparison factor in section 4.7 .
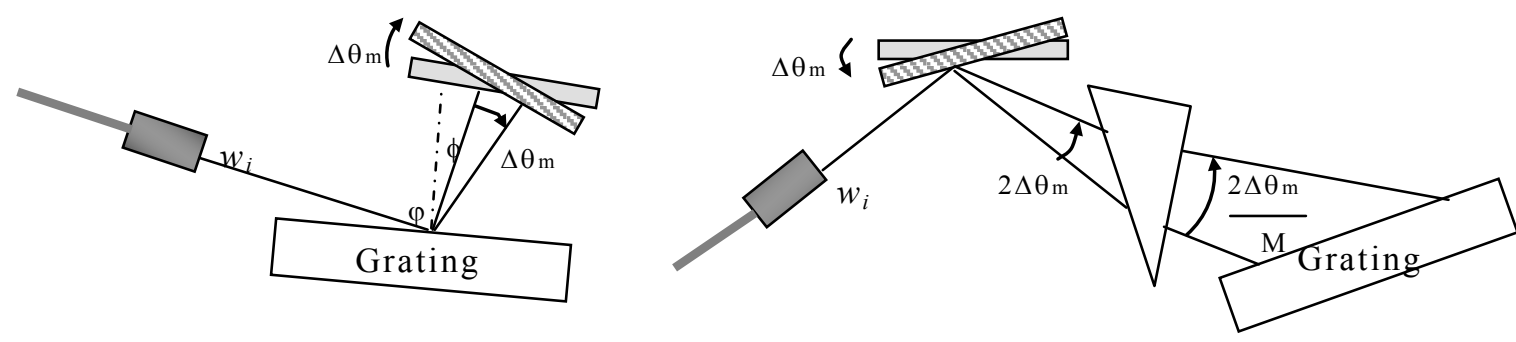

Figure 4.2 Comparison of Littman configuration (left) and Littrow configuration (right)
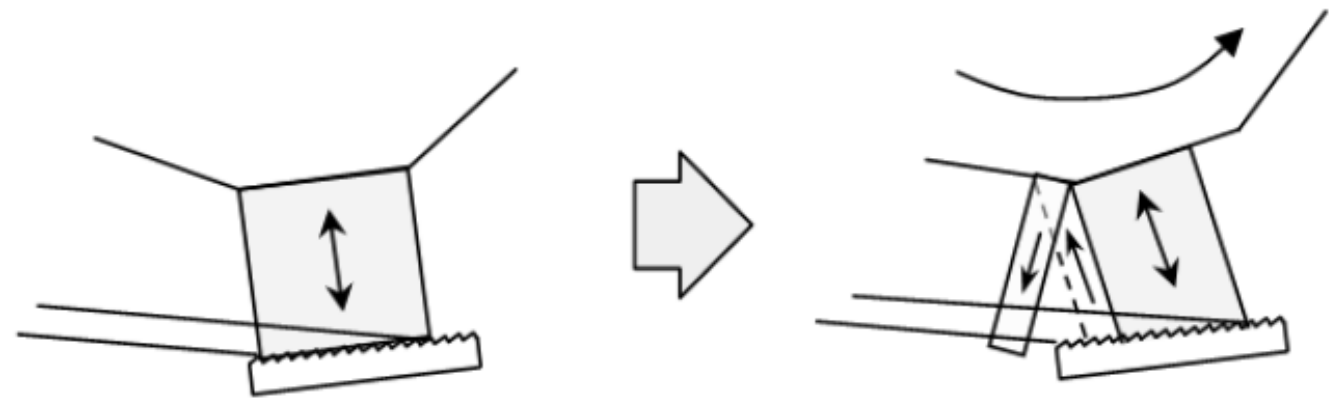

Figure 4.3 Beam walk-off issue: As the polygon rotates, a portion of the beam is cutoff during the transition to a next facet of polygon scanner 


\subsection{Spatial Spectral shaping technique}

It is possible to control wavelength dependent loss in a cavity by an aperture with a tailored shape as shown in Fig.4.4. A knife-edge shaped aperture having different edge profile along the position is placed at the wavelength dispersed space in front of diffraction grating, and which attenuates the power level at different wavelength so that arbitrary shaping of output scanning spectrum is realized. Designing the shape of this aperture tailors the desired profile of spectrum.

In general with SOA, the higher the current is injected, the wider the gain bandwidth becomes. On the other hand, in the swept source configuration, the optical power level over the scanning range increases as the injection current increases. In the extremely high power emission regime, the chip facet of the SOA is damaged or degraded by so called catastrophic optical damage (COD). A primary mechanism of COD is known to be a thermally induced material meltdown due to high optical and current densities at the output facet [81-83]. Especially, AlGaAs multiple-quantum well is known to have lower COD threshold. For this reason, just increasing the current cannot realize the wider scanning range.

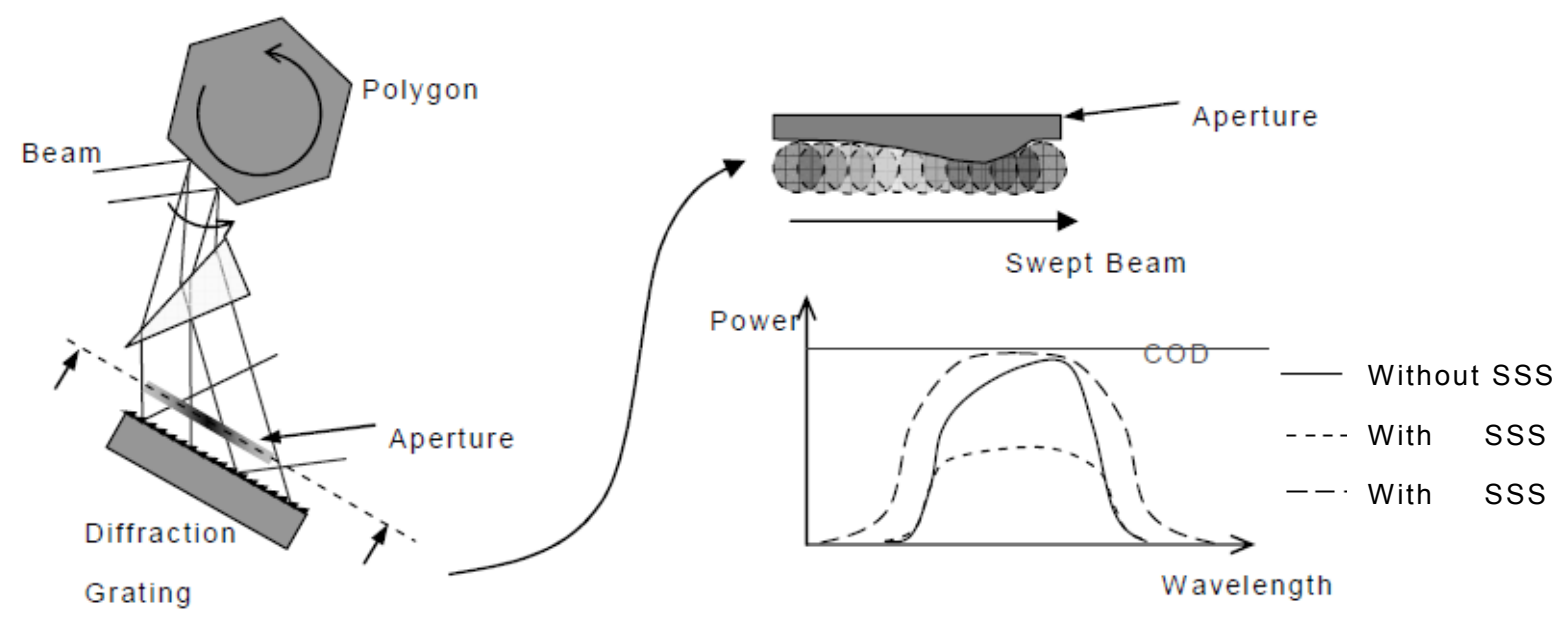

Figure 4.4 Spatial Spectral Shaping(SSS) Technique

By using spectral shaping technique one can overcome this limitation. COD-limited peak power can be clipped by adding attenuation at this region while increasing the gain around at edges of the scanning range. As a result, a wider and symmetric Gaussian-like shape profile can be provided. ASS-technique is possible in not only the aperture but also ND filter and thin film filter.

Figure 4.5 shows the output spectrum measured with the optical spectral analyzer in peak hold mode. Ripple that is observed around $1360 \mathrm{~nm}$ corresponds to the water absorption 
peaks. The peak power of the source was $20 \mathrm{~mW}$, tuning range was over $160 \mathrm{~nm} \mathrm{FWHM,} 170$ $\mathrm{nm}$ in total from $1230 \mathrm{~nm}$ to $1400 \mathrm{~nm}$ as shown in Fig.4.5 and Fig.4.6, and coherence length was $3.6 \mathrm{~mm}$. Fiber ring cavity for this laser consists of single mode fiber with single mode cut-off wavelength at $1260 \mathrm{~nm}$. Therefore, it is notable that the normal SMF fiber excites the secondary lateral mode when the propagating light is below cut-off wavelength of $1260 \mathrm{~nm}$, and which could degrade the coherence of the measurement as a result. In order to avoid this multi-mode propagation at shorter wavelength range, we spliced the output fiber with HI1060 type fiber that have cut-off wavelength at around $960 \mathrm{~nm}$ so that it allows only single lateral mode propagation over $1230 \mathrm{~nm}$ to $1400 \mathrm{~nm}$ at optical output. The wavelength range of subsequent OCT system including an interferometer and a probe should be matched in this aspect.

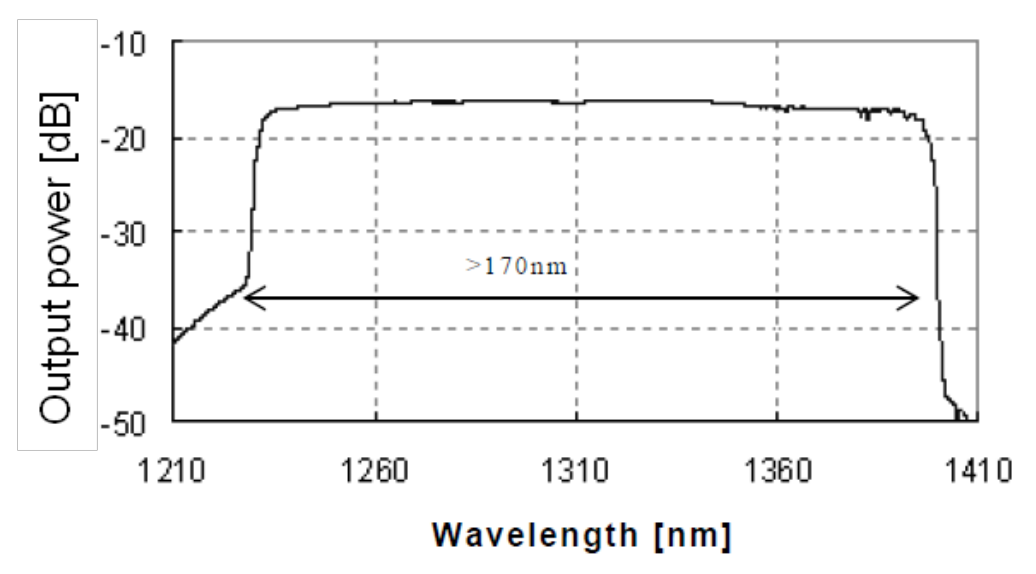

Figure 4.5 Output spectrum of the laser at $20 \mathrm{kHz}$ scanning rate measured with optical spectrum analyzer in peak hold mode

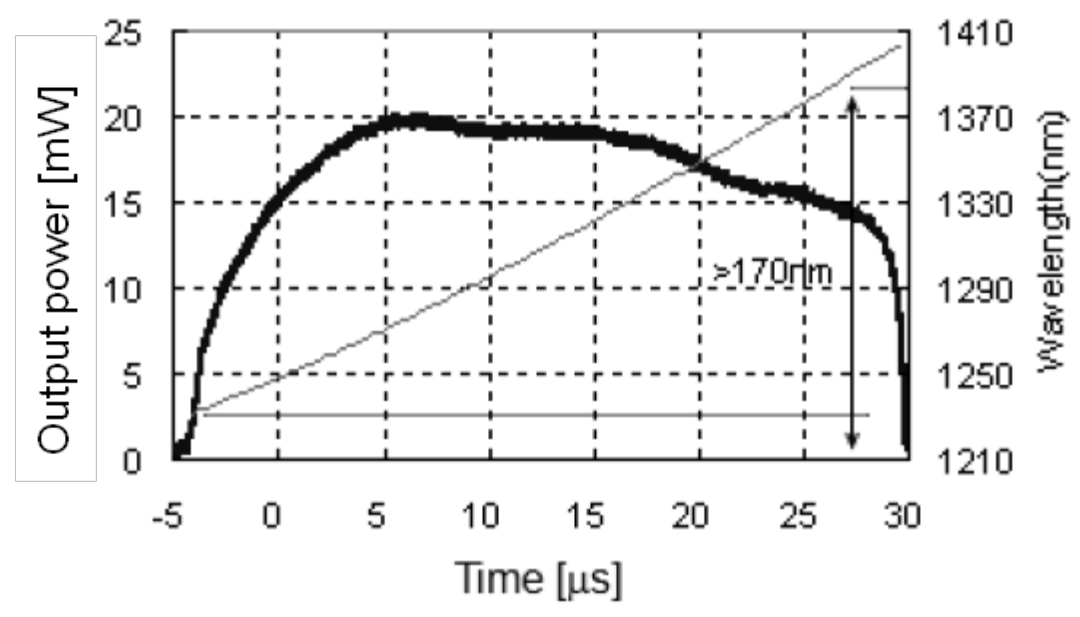

Figure 4.6 Temporal intensity and wavelength profile 


\subsection{Wide range sweep by injection current modulation}

For conventional instrument type tunable lasers, there is a function to control injection current of gain chip or LD so that the output optical power remains in constant over tuning range. This is usually called "automatic power control-APC" mode. The reason why we cannot do this and need to take a measure like we did in the previous section, i.e. spatial spectral shaping is mainly because the sweep of the source is intrinsically "free running" in a way. Polygon scanner keeps rotation at quite fast speed so there is no simple way to actively monitor the power and feedback to the power level. However, since the temporal power profile is quite repeatable over a sweep because of stable polygon scanner's rotation, we can take advantage of this repeatability instead. If the temporal power profile is given, we can insert a temporal offset to the injection current with using calibration table over and over for each sweep. In order to realize this, we need to synchronize the timing of the offset by using the integrated start trigger signal as shown in Fig.2.1. We have designed the LD driver circuitry that can initiate temporal offset to the injection current with external trigger input. Temporal injection current profile has a nearly inverse of optical output power profile. In this way gain at band edges can be lifted by increase of the injection current, thus broadening the total swept range in terms of FWHM. This technique is effective way especially when the maximum drive operation is not only limited by COD but also limited by damage threshold of injection current at the peak or center of wavelength range. $1 \mu \mathrm{m} \mathrm{SOA}$ is typical of this example.

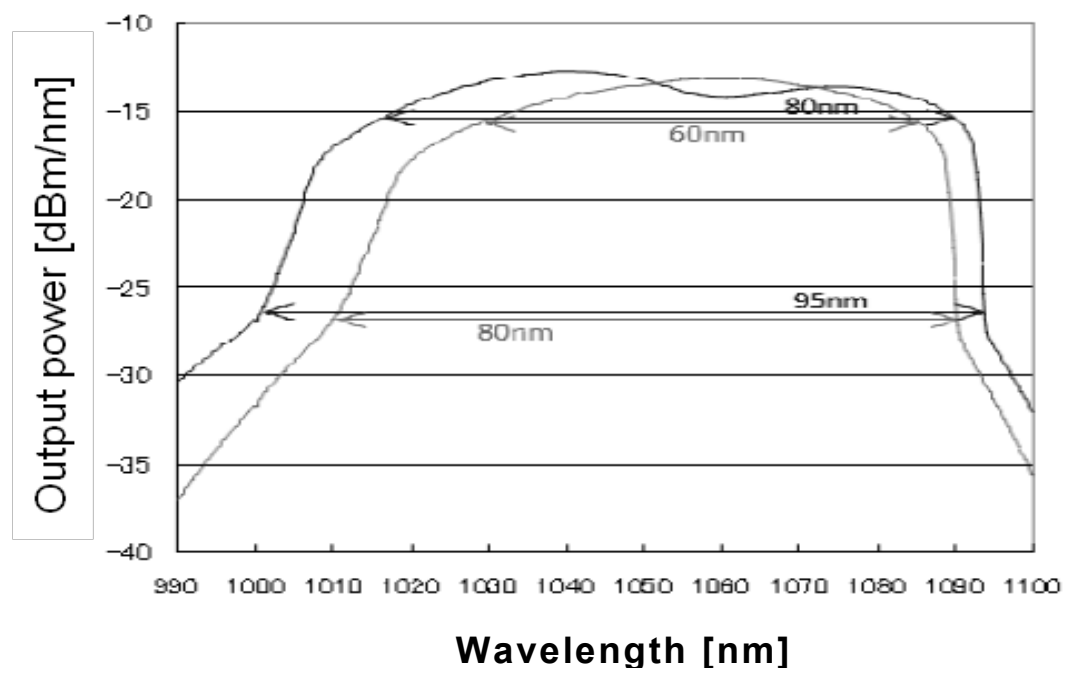

Figure4.7 Peak-hold mode spectrum with OSA 


\subsection{Double pass configuration}

Further improvement of coherence length can be realized by applying a double pass configuration to the same geometrical arrangement. The free space four ports circulator block as proposed in Fig. 4.8 is designed to direct the light beam twice from the collimator to the diffraction grating via polygon scanner in the exactly same incident angle. Subsequent beam in identical polarization state that exits from this block has an offset beam shift of about $2 \mathrm{~mm}$ in perpendicular direction to the beam deflection plane (details depicted in next page). In results, the filter bandwidth is narrowed by about a factor of two; this is comparable to that of a Littman configuration of grating filter. Figure 4.9 (a) shows narrowing of the spectral bandwidth of tunable filter and Fig. 4.9 (b) shows the improvement of coherence length. The measured coherence length when using double pass configuration was found to be $7 \mathrm{~mm}$ which is twice as large as that with single pass configuration.
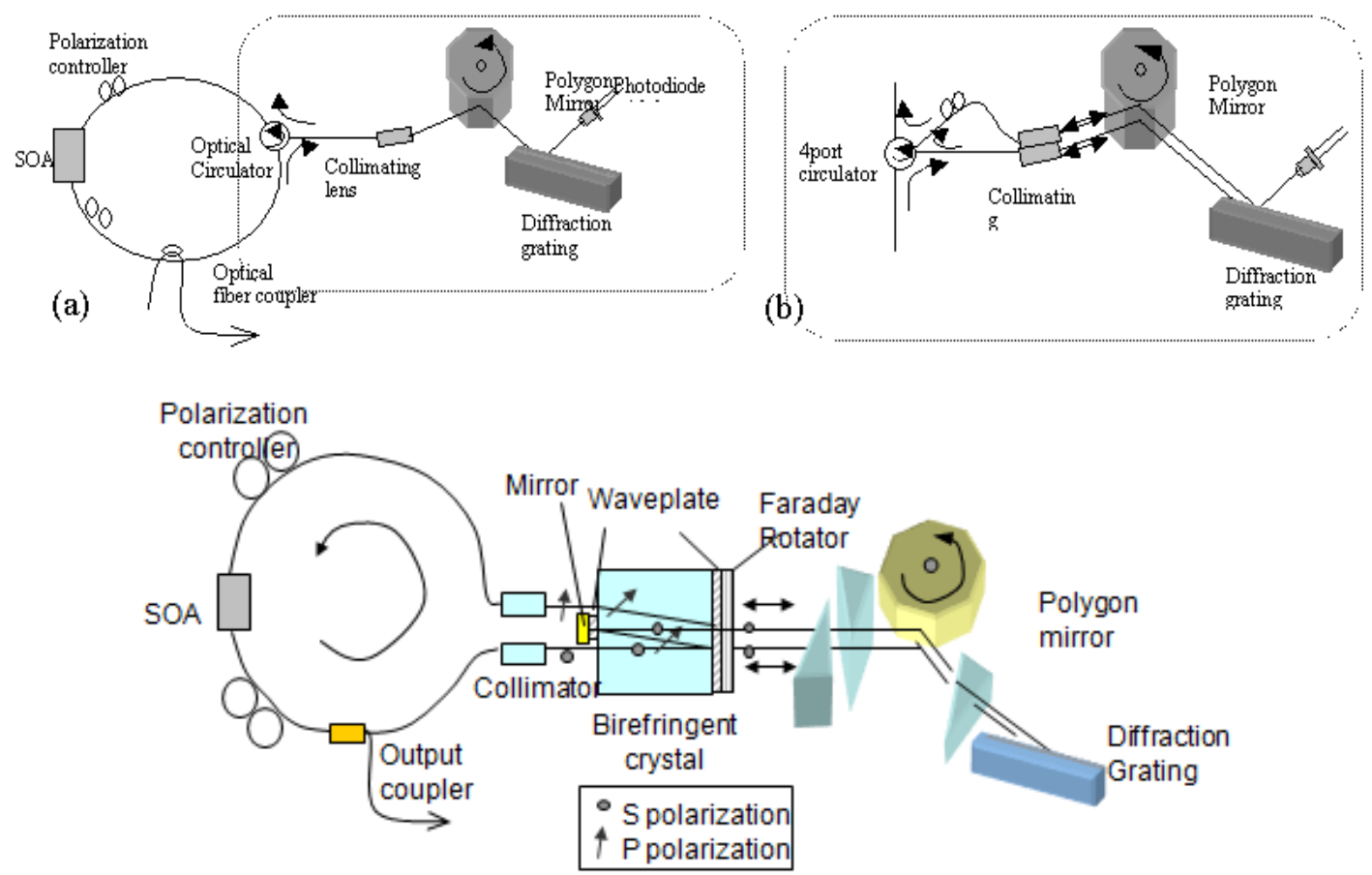

Figure 4.8 Swept source in a double pass Littrow configuration with using a free spcase ciruculator block

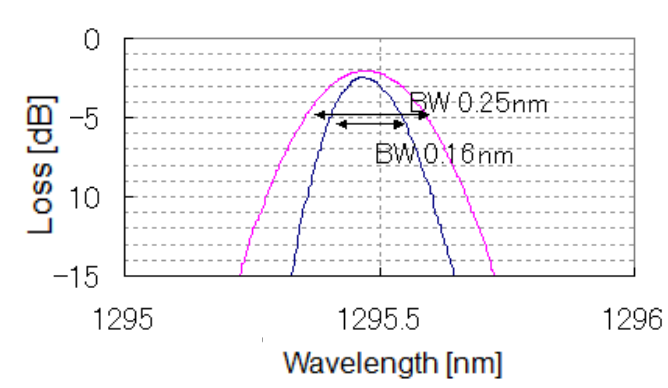

Figure 4.9 (a) Filter bandwidth

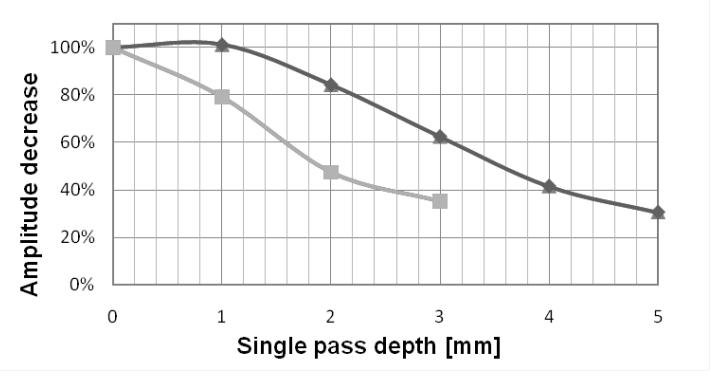

(b) Amplitude of interference fringes vs. depth 


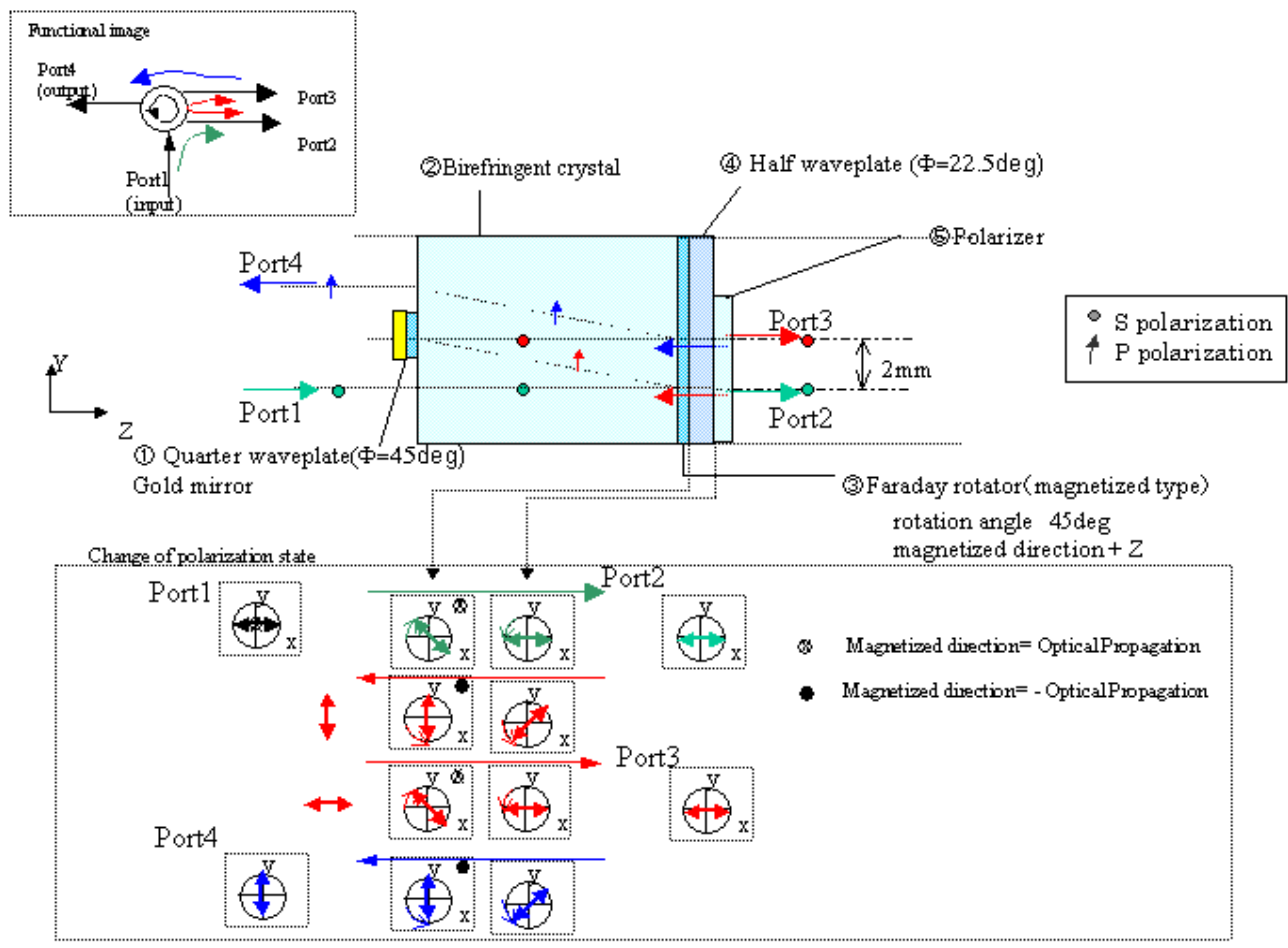

Figure 4.10 Mechanism of single polarization recovery module

The structure of the device is explained as follows. The birefringent crystal Rutile is used as a polarization beam splitting device. A faraday rotator and zeroth order half-waveplate of quartz are bonded to the end face of birefringent crystal and a quarter waveplate with gold mirror on the incident facet in between entrance beam and exit beam. S polarized input light travels straight through the birefringent crystal, at the exit end the half waveplate rotates the polarization $+45 \mathrm{deg}$ with respect to the propagation of the light, then the Faraday rotator rotates $45 \mathrm{deg}$ with respect to the direction of magnetization regardless of optical propagation. Thus, input beam via port 1 in $\mathrm{S}$ polarization propagates straightforward through the birefringent crystal to the end where polarization rotates $+45 \mathrm{deg}$ and $-45 \mathrm{deg}$ at Faraday rotator and half waveplate, respectively, hence remains in the initial state of polarization exiting at port2. The light is retro-reflected from the grating upon entering the splitter at port 2 the polarization is rotated $-45 \mathrm{deg}$ by the half waveplate and by -45 deg by Faraday rotator, a total of $-90 \mathrm{deg}$ change, converting the light from $\mathrm{S}$ to $\mathrm{P}$ polarization. Then P polarization, extra-ordinary ray, propagation is offset with respects to the input light path, propagating to the quarter waveplate and reflected by gold mirror where it rotates to $\mathrm{S}$ polarization again. The subsequent process is exactly the same as the first pass just shifted in the optical axis. The beam exits through port 3 in $S$ polarization, and after reflected by diffraction grating for second time, it is routed back to Port 4 in the same process but only in P polarization state to the output collimator fiber. Polarization controllers within single mode fiber ring cavity are used to adjust the two orthogonal polarization states at port 1 and 4 so that a single polarization state is locked when lasing occurs. 


\subsection{Double reflection configuration}

High-speed wavelength swept-lasers have been widely adapted to applications such as optical coherence tomography (OCT), fiber sensor system, gas sensing, and surface profiling. In a swept-source OCT (SS-OCT) system, requirements for the swept rate and swept range as well as coherence length are slightly different depending on applications. For example, in cardiovascular imaging, swept rate of over $30 \mathrm{kHz}$ or higher is desired because three-dimensional volume acquisitions should be completed within several seconds during the saline flush inside the coronary. Also in ophthalmology applications, both faster and wider sweep is desired to be able to acquire high-resolution image without the influenced of motion artifact due to eye-movement.

Previous works suggest that polygon-scanner based wavelength swept sources in various configurations can provide the high-speed swept rates while maintaining practical swept range and coherence length [51-55]. The use of polygon scanner ensures the stable sweep over long term with high reliability as well as unidirectional saw tooth sweep, which is convenient for OCT system design. In this case, Finesse of the tunable filter inside the swept source determines the swept range and coherence length corresponding to instantaneous linewidth.

Design trade-off between these three parameters; wavelength range, tuning speed and coherence length, are attributed to the mechanical limitation of polygon scanner. Maximum rotation speed of industrially proven polygon scanners ranges about 40,000 to 45,000 rpm with a scanner mirror diameter of $60 \mathrm{~mm}$. The swept rate is simply determined by the facet number of polygon mirror. The deflection angle is proportional to the swept range and widening the illuminating beam width onto the diffraction grating narrows filter bandwidth. So the swept range and filter bandwidth are automatically restrained by deflection angle and facet width, both of these are reduced as the facet number increases. In one polygon-based scheme, a telescopic arrangement is used to convert diverging angular dispersion from the grating into converging angular dispersion so that polygon scanner reflects back the imaged beam on the facet, the rotation of which tunes the wavelength unidirectionally [47,51]. The combination of telescope configuration and additional multiple reflections [54] has been shown to increase finesse at higher swept rates. This approach, however, may have a problem in robustness in terms of manufacturing process because of complex and bulky optics to configure telescopes in the filter. We proposed a swept source using a simplified Littrow arrangement with a prism expander that demonstrates $120 \mathrm{~nm}$ swept range at $20 \mathrm{kHz}$ swept rate [48, 84]. However, this approach has limitation in swept range when the swept rate increases up to $50 \mathrm{kHz}$ range. While, a swept source providing $91.6 \mathrm{~nm}$ at $50 \mathrm{kHz} \mathrm{swept}$ rate has been recently presented which doesn't incorporate telescope configuration such as 
in ref.[55] and overcome the limits of Littrow arrangement. High Finesse was expected by utilizing multiple reflections from the polygon facets and grating illuminations. However, in this scheme, there is concern that the beam clipping at the polygon facets broadens the linewidth and excessive loss due to multiple diffractions at the grating reduces the swept range.

In this section, a further simplified configuration without telescopic configuration is proposed that matches the theoretical Finesse demonstrated by ref. [55] with improved performance, minimizing the effect of beam clipping. The schematic diagram of the filter configuration is shown in Fig.4.11.

The beam from the collimator is expanded to the size comparable to the size of polygon facet and deflected by the rotation of polygon scanner. Unlike in a conventional filter where the deflected beam is projected directly onto the diffraction grating, we have used instead an additional mirror placed in front of polygon scanner that returns the beam to the scanner for a second deflection. Wavelength is tuned from shorter to longer wavelength by polygon's rotation, by using a second deflection the total deflection angle of the beam is doubled compared to that of a single deflection. The position of the additional mirror is calculated to return the beam to the center of the second facet over the course of one complete wavelength scan, thereby synchronizing first and second deflections and eliminating beam clipping. In this way we have achieved a twofold increase in FSR compared to a single scanner deflection, without beam clipping at the second facet.
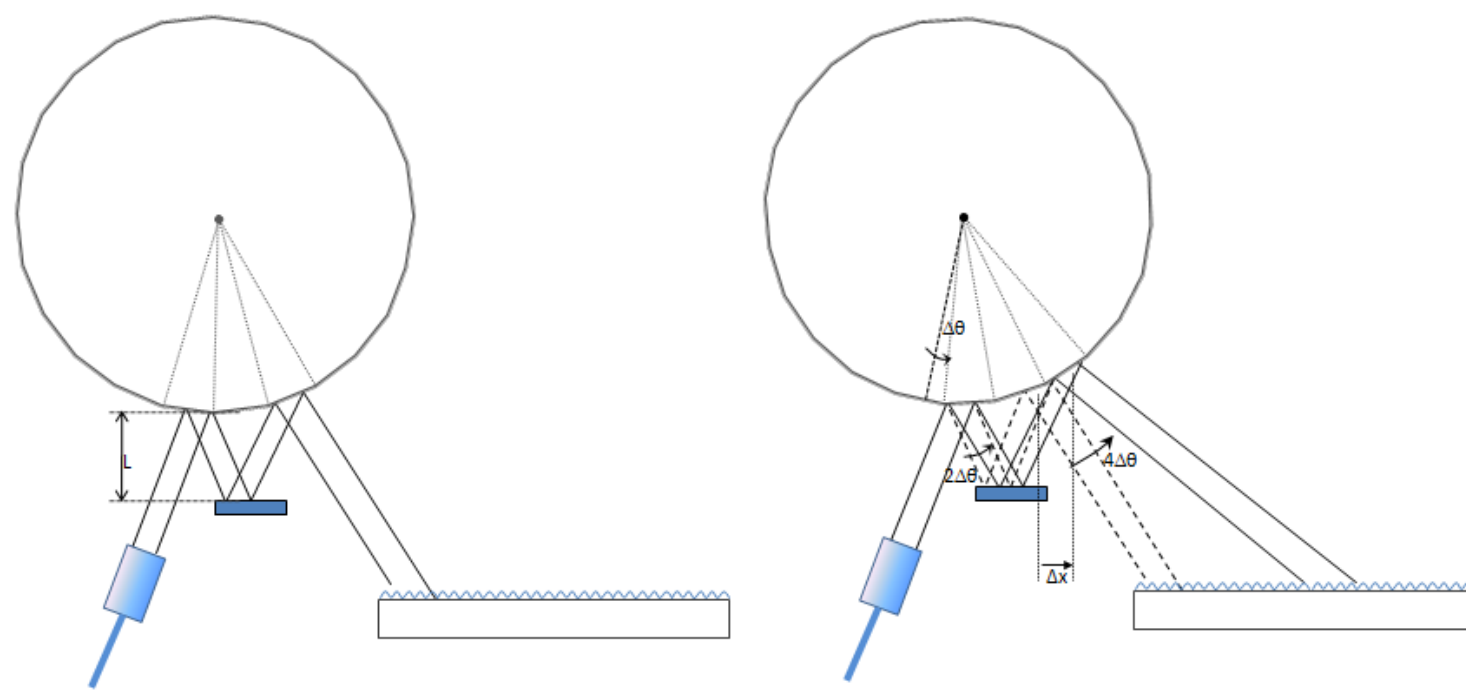

Figure 4.11 Synchronous double-deflection 
We derive the optimum geometry for the filter by equating the beam shift; $d x=4 \Delta \theta \mathrm{L} / \cos \phi \cos (\phi+2 \Delta \theta)$ and polygon facet width $\mathrm{d}=2 \pi / \mathrm{N}$ as a function of polygon mirror diameter $\mathrm{D}$, where $\Delta \theta$ is the partial rotation angle per facet and $\mathrm{f}$ is incident angle to the first facet and $L$ is the distance between external mirror and the facet.

Condition yields the simple relation:

$$
\mathrm{L}=\cos \phi \cos (\phi+2 \Delta \theta) \mathrm{D} / 8=\mathrm{D} / 8
$$

Here, the size of external mirror $\mathrm{d}_{2}$ should be;

$$
\mathrm{W}+d x / 2<\mathrm{d}_{2}<\mathrm{L}(\tan \phi+\tan (\phi+2 \mathrm{~m} \Delta \theta))
$$

so that the beam is not clipped by the mirror's edges, where the second projection on the polygon is on the $m$ th facet from the first facet of reflection.

In this demonstration, we use a polygon with diameter of $60 \mathrm{~mm}$, L was calculated to $7 \mathrm{~mm}$ when the beam returns second nearest neighbor facet. The mirror is fixed at the window of the polygon scanner casing.

FSR and filter bandwidth of the tunable filter is given in Eqs. (4.8), (4.9)

$$
\begin{aligned}
& \Delta \lambda=8 a \cos \theta_{0} \cdot \Delta \theta \\
& \delta \lambda=\frac{4 \sqrt{\ln 2} \lambda a \cos \theta_{0}}{\pi W}
\end{aligned}
$$

Finesse is given by Eq. (4.10) and theoretical value assuming no beam clipping at first facet is 1400 which is identical to that in ref. [55] when facet number is set to 70 .

$$
F=\frac{4 \pi^{2} W}{\sqrt{\ln 2} \lambda_{o} N}
$$

The configuration for the swept tunable laser is shown in Fig.4.12. The gain medium used was a semiconductor optical amplifier, SOA (COVEGA BOA1132) with an emission bandwidth of over $160 \mathrm{~nm}$ centered at $1310 \mathrm{~nm}$. Polygon scanner rotation was set to 43000 $\mathrm{rpm}$ or $717 \mathrm{rev} / \mathrm{sec}$ to provide $50 \mathrm{kHz}$ swept rate with 70 facets of polygon mirror. The collimated beam from the collimator was expanded with a series of prism expanders to the $1 / \mathrm{e}^{2}$ width of $1.8 \mathrm{~mm}$, set smaller than a facet width of $2.7 \mathrm{~mm}$ to reduce the beam clipping. A second beam expander is used between the scanner and diffraction grating with 1312 line/mm constant. Although the placement of two expanders reduces the diffraction angle range at the grating, it contributes to the narrowing of the filter bandwidth by expanding the beam. Thus, overall finesse is the same. The combination of the use of expanders and selection of grating constant gives the flexibility of design to optimize scan range and linewidth as well as enabling k-linear sweep [84] eliminating the need of k-triggering in 
SS-OCT system. The designed filter FSR is $124 \mathrm{~nm}$ with a bandwidth of $0.134 \mathrm{~nm}$, corresponding to coherence length of $5.6 \mathrm{~mm}$.

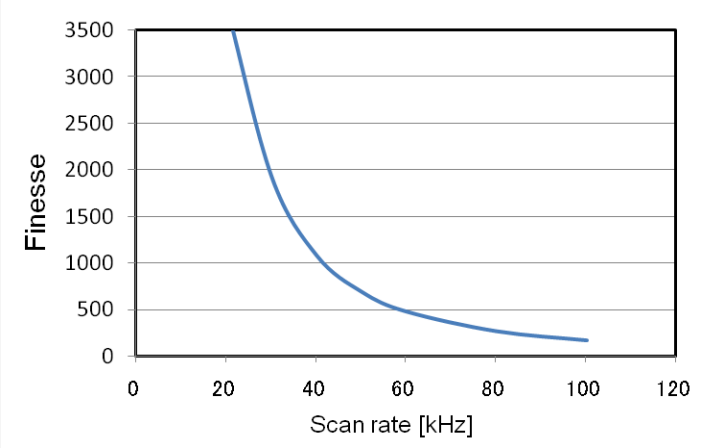

Figure 4.12(a) FSR vs. Scan rate

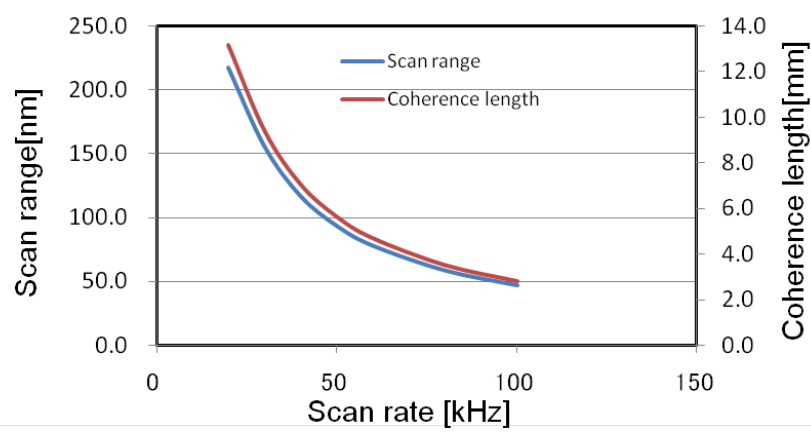

(b) Scan rage, Coherence length vs. Scan rate

The fiber ring used in this study is configured using components with polarization maintaining fiber (PMF) pigtails so that polarization controllers for adjusting the polarization states with respect to the tunable filter and an SOA are eliminated. As a result, the fiber ring cavity can be spliced into a cavity as short as $1.3 \mathrm{~m}$ in optical length. This way suppresses the minimum wavelength shift per round trip. Moreover, the use of PMF will ensure the long-term reliability of the lasing performance while SMF type might have a variation of polarization over long term and under mechanical stress that affects on the lasing spectrum.

The slow axis of the collimator lens is aligned in the polarization for the highest diffraction efficiency, i.e. S-polarization (or TM) with respect to the diffraction grating. Lasing output light is coupled to the slow axis of PMF. Here, since the amplified spontaneous emission from the SOA is also coupled into the orthogonal axis of PMF: i.e. fast axis, it creates beating noise due to cross polarization interference when it is connected to the OCT interferometer with single mode fiber which may result in the ghost image. In order to avoid this beating noise, a fiber polarizer is inserted at the output fiber to filter out the orthogonal polarization, or increasing the polarization extinction ratio over $40 \mathrm{~dB}$. The measured degree of polarization at output is over $99 \%$ polarized light. 


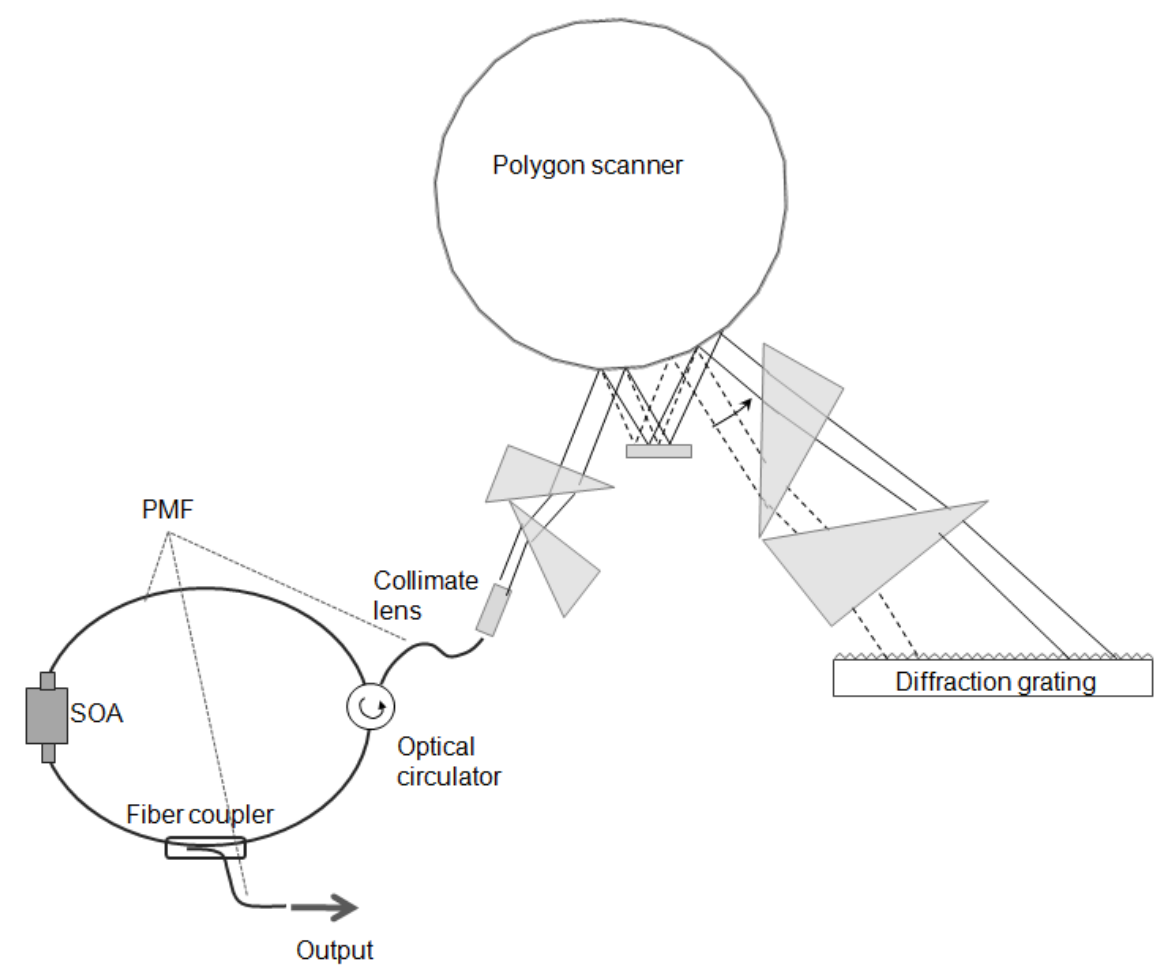

Figure 4.13 Laser configuration

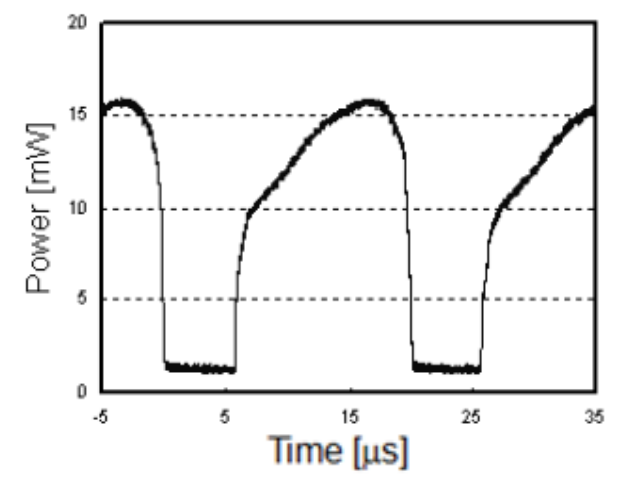

Figure 4.14 (a)Output power spectrum

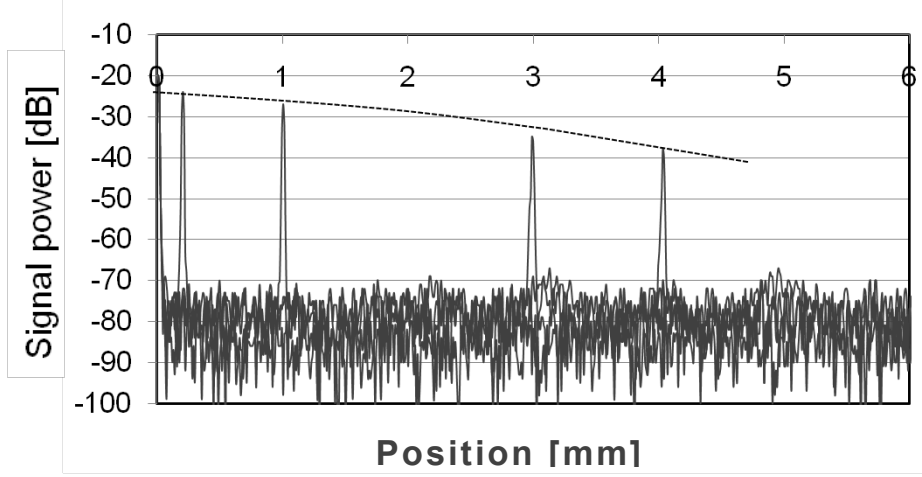

(b)OCT signal power at different depths

Figure 4.14 shows the laser output temporal spectrum and OCT signal power at different depth positions with wavelength rescaling process. Peak power was $15 \mathrm{~mW}$ and the edge-to-edge swept range was $99 \mathrm{~nm}(1260 \mathrm{~nm}$ to $1359 \mathrm{~nm})$ and the duty cycle was $78 \%$, which agrees well with theoretical FSR of the tunable filter. Imperfect alignment of the external mirror and beam clipping by the edges of prism expanders account for difference between theoretical and measured swept range. Signal drops $6 \mathrm{~dB}$ at $2.7 \mathrm{~mm}$ corresponding to the coherence length of $5.4 \mathrm{~mm}$ and instantaneous linewidth of $0.139 \mathrm{~nm}$, which is in good agreement with theoretical value owing to the cavity length as short as $1.8 \mathrm{~m}$ in optical 
length. Axial resolution of about $12 \mu \mathrm{m}$ can be confirmed from the OCT signal power spectrum at $1 \mathrm{~mm}$.

An actual OCT image was obtained to demonstrate the imaging capability of the source. OCT system consists of a symmetrical Mach-Zehnder interferometer with a balanced detector connected to the DAQ board with $100 \mathrm{MS} / \mathrm{s}$. An image was constructed in 250 A-lines/frame at $50,000 \mathrm{~Hz}$ swept rate of the proposed source. Lateral resolution set by the probe is $25 \mu \mathrm{m}$. Figure 4.15 shows the image of skin at the finger. Sweat ducts and epidermis, dermis are clearly observed in the image of $250 \times 1048$ pixels.

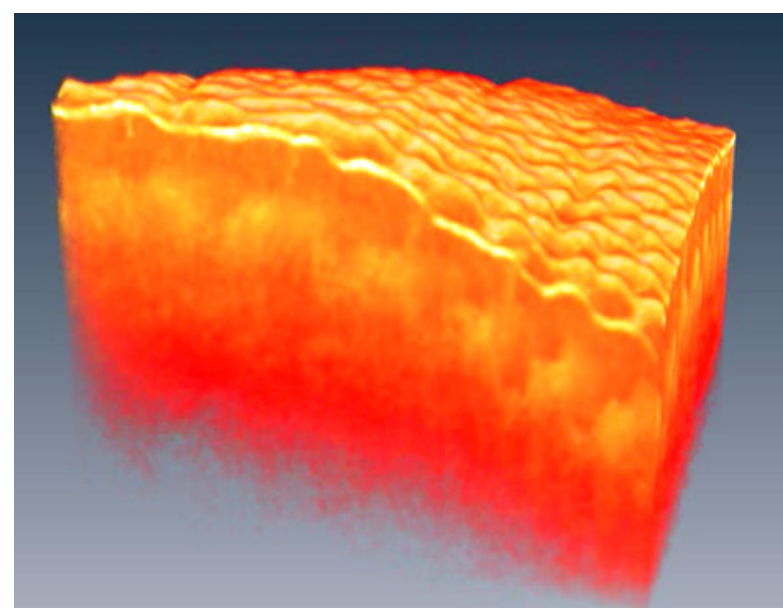

(a) $3 \mathrm{D}$ image (Image size: $250 \times 250 \times 1024$ voxel)

Figure 4.15 OCT image of finger

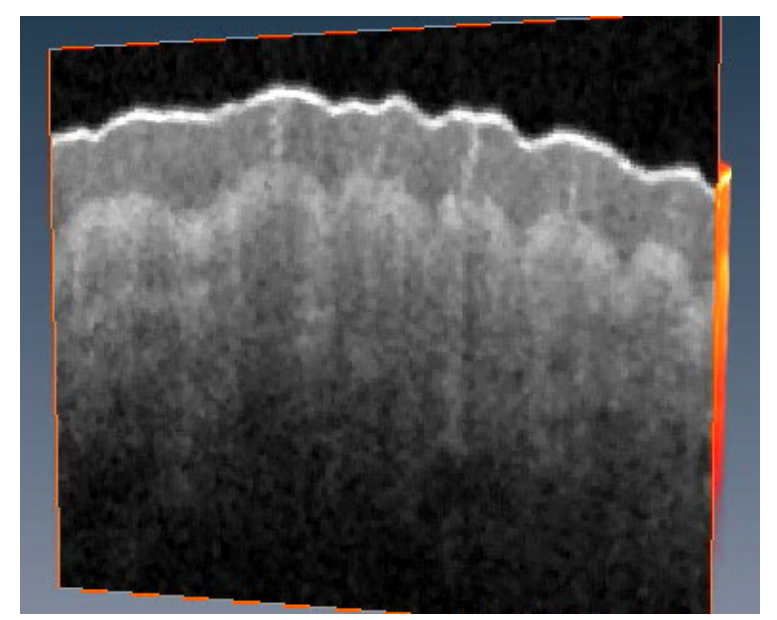

(b)Sliced 2D sectioning (250x1024 pixel) 


\subsection{Summary}

In this chapter, various approaches to overcome the design trade-off between swept range and coherence length at high speed sweep were proposed. We proved that spectral shaping and injection current modulation are effective to take full advantage of wide gain band of SOA. Both techniques can expand the bandwidth without worrying the issue of COD. Maximum of $170 \mathrm{~nm}$ tuning range was demonstrated with the above method.

Double pass and reflection configuration were also proposed and found that both configurations are effective to countermeasure the design limit of tunable filter using diffraction grating and polygon scanner. Double reflection configuration enabled $100 \mathrm{~nm}$ wide range sweep at $50 \mathrm{kHz}$ swept rate while maintaining practical $5 \mathrm{~mm}$ coherence length during the sweep. $50 \mathrm{kHz}$ fast sweep proved the fast acquisition of three-dimensional tomograph. 


\section{Chapter 5 OCT system demonstration and clinical studies}

In this chapter, SS-OCT system is disclosed more in detail than in previous chapters, with introductory discussion of the sensitivity advantage over conventional time-domain system that was already given in previous works [18-20]. Secondly, the system configuration and signal calculation algorithm are introduced. Following sections will show some of example OCT images taken with using the fabricated swept source in the previous chapters. Some works are collaborative work with other institutions for clinical studies in oral tissues and anterior chamber. All clinical images shown here are approved by ethical committee of each institution with informed consent from volunteer patients. In the last section, novel optically modulated MEMS scanning probe is introduced for endoscopic OCT applications.

\subsection{Principle of SS-OCT}

Sensitivity of the Fourier-domain system

Figure 5.1 shows basic schematic of SS-OCT. The output of the swept source is divided into a reference arm and a sample arm. Backscattered and recoupled light from the sample arm creates interference with a light reflected from reference arm. Interferogram is scanned over frequency domain with the wavelength sweep over and detected with a photoreceiver. A-scan or A-line signal along the depth is obtained by fast Fourier transform (FFT) or discrete Fourier transform (DFT) on the time-sampled signals [85].

Raw signal is expressed as

$$
i_{\text {det }}(t)=\frac{\eta q}{h v}\left(P_{r}+P_{o} \int r^{2}(z) d z+2 \sqrt{P_{r} P_{o}} \int r(z) \Gamma(z) \cos (2 k(t) z+\phi(z) d z)\right.
$$

where $\eta, q, h v$, are detector sensitivity, quantum electric charge, single photon energy, respectively, and $\operatorname{Pr}$, Po are optical power reflected from the sample, optical power illuminating the sample, respectively. 
The third term represents the OCT signal and the first and second DC components. G is the coherence function of the instantaneous laser output, which is related to the linewidth and coherence length.

For a swept-laser source with a Gaussian spectral envelope, the axial resolution is given by

$$
\delta z=\frac{2 \ln 2}{\pi} \frac{\lambda_{0}^{2}}{n \Delta \lambda}
$$

Where $\lambda_{0}$ is the center wavelength, $\Delta \lambda$ is the FWHM of swept range, and $\mathrm{n}$ is the group refractive index of the sample. The depth range $\Delta z$ is given by

$$
\Delta z<\frac{\lambda_{0}^{2}}{4 n \delta \lambda}
$$

where $\delta \lambda$ is the sampling interval of wavelength and Ns is the number of samples within FHWM of $\Delta \lambda$. Sampling interval should be smaller than instantaneous linewidth of the source. $\delta \lambda$ is typically less than half of instantaneous linewidth to keep the usable depth range.

$$
c l=\Delta L \times 2=\frac{2 \ln 2}{\pi} \frac{c}{\delta v}
$$

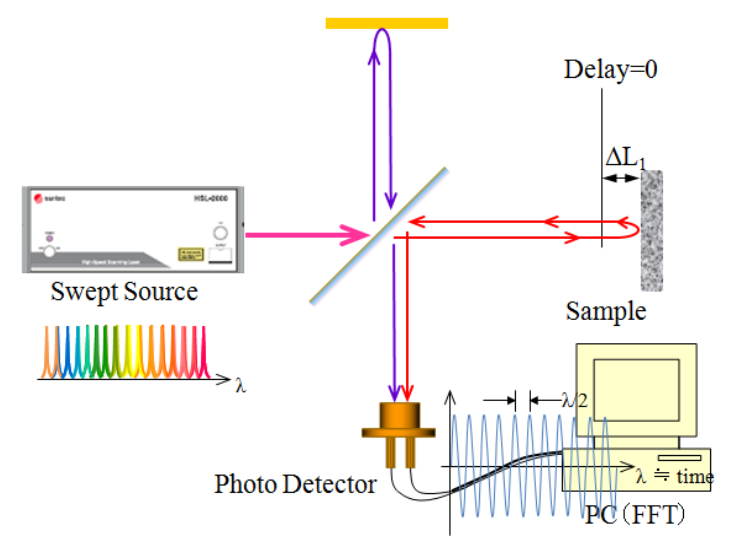

Figure 5.1 OCT interferometer

\section{Detection sensitivity}

The OCT signal current at the detector can be expressed as

$$
i_{s}(t)=\frac{\eta q}{h v} 2 \sqrt{P_{r} P_{s}} \cos \left(2 k(t) z_{0}\right)
$$

Where we assume the linewidth is sufficiently narrow so that $\Gamma(z)=1$ within the depth range, and $\mathrm{P}_{\mathrm{s}}=\tau_{2} \mathrm{P}_{0}$ denotes the optical power reflected from the sample at the photodetector. While, current for noise components can be expressed as [85] 


$$
\left\langle i_{n}^{2}(t)\right\rangle=\left(i_{t h}^{2}+2 \frac{\eta q}{h v}\left(P_{r}+P_{s}\right)+\left(\frac{\eta q}{h v}\right)^{2} \operatorname{RIN}\left(P_{r}+P_{x}\right)^{2}\right) B W
$$

where each term represents thermal noise, shot noise, the relative intensity noise (RIN), respectively.

OCT signal is FFT or DFT of signal current $\mathrm{i}(\mathrm{km})$;

$$
F(z)=\sum_{m=0}^{N_{s}-1} i\left(k_{m}\right) \exp \left(-2 j \pi l m / N_{s}\right)
$$

Note that in Fourier domain, absolute square of the peak value of $\mathrm{F}_{\mathrm{s}}$ at $\mathrm{z}_{0}$ is proportional to the reflectivity. The fact that Parseval's theorem, $\sum F^{2}=N_{s} \sum i^{2} \quad$ holds for both signal and noise leads to the relation of SNR in comparison with SNR of time-domain ranging [23];

$$
(S N R)_{F D}=\frac{\left|F_{s}(z)\right|^{2}}{\left\langle F_{n}^{2}\right\rangle}=\frac{N_{s}}{2}(S N R)_{T D}=\frac{N_{s}}{2} \frac{\left\langle i_{s}^{2}(t)\right\rangle}{\left\langle i_{n}^{2}(t)\right\rangle}
$$

$\mathrm{N}_{\mathrm{s}}$ is the number of sampling points which should be equal or greater than a half of the number of frequency resolvable points, i.e. $2 \mathrm{~N}_{\mathrm{s}}=\Delta \lambda / \delta \lambda$, which is the ratio of tuning range to the linewidth. For an example, when $\Delta \lambda=100 \mathrm{~nm}$ and $\delta \lambda=0.05 \mathrm{~nm}$, then SNR of Fourier-domain OCT is $\mathrm{x} 1000$ or $30 \mathrm{~dB}$ better than that of time-domain OCT in ideal or in a simplified estimation. In reality, duty cycle of tuning is less than $100 \%$, and source's spectrum is in Gaussian profile and sampling range spans beyond FWHM of the source spectrum. In this case, Ns is the number of sampling points within FWHM of the source and the optical powers, $\operatorname{Pr}$ and Ps is the time-averaged value over on sweep cycle. For a shot noise limited system, eq. (5.6) and (5.8) with $\operatorname{Ps}<<\operatorname{Pr}$ leads to

$$
(S N R)_{F D}=\frac{\eta P_{s}}{h v f_{A}}
$$

where $f_{\mathrm{A}}$, is the A-line rate or equal to swept rate of the source. Hence, the effective detection bandwidth of the system is equal to the A-line rate instead of detector's bandwidth.

Finally, the sensitivity is expressed as

$$
\text { Sensitivity }[d B]=-10 \log \left(\frac{\eta P_{s}}{h v f_{A}}\right)
$$

In practical system, to reduce the source noise, dual balanced detection is employed [86]. The differential detection gives $3 \mathrm{~dB}$ benefit in dynamic range as well as suppression of RIN of the source, self-interference noise [19], and fixed-pattern noise or background noise by its common-mode noise rejection property. 
However, cross-beating noise such as polarization cross beating or slow ripple on the gain spectrum of SOA results in fixed ripple or noise on the signal spectrum which ends up in fixed alias noise in the OCT image. Such alias can be eliminated by unwrapping the noise component from the signal [39]. But it is desired to have smooth sweep spectrum of the source without such ripple and polarization noise in the first place so that you can avoid unnecessary post-processing. 


\subsection{OCT system configuration}

An SS-OCT system with a swept laser source is shown in Fig.5.4. Output beam from the source is introduced into a fiber-based Mach-Zehnder interferometer with a 1:99 1x2 fiber coupler, two circulators, and a 50:50 2x2 fiber coupler. The through port with 90\% coupling ratio of the first coupler is connected to a probe arm though a three-port circulator. Probe arm comprises a collimator lens and a galvano scanner, achromatic lens. The lateral resolution is determined by a diameter of collimator lens D, and focal length of achromatic objective lens f, as given in Eq. (5.11).

$$
d x=\frac{4 f \lambda}{\pi D}
$$

The $1 \%$ port of the first coupler is connected to the reference arm which is configured symmetrically with a same circulator, collimator and achromatic lens as those used in the probe arm. Probe and reference beams are recoupled by $2 \times 2$ coupler and two outputs are fed into the balanced photoreceiver (newfocus 1544) with a band pass of $80 \mathrm{MHz}$. A variable optical attenuator and polarization controllers are used to adjust the dynamic range of interference signal and optical delay line combined with the objective lens and a reflective mirror is used to adjust the zero delay to match the focus area of the probe arm. An additional visible laser light at $633 \mathrm{~nm}$ is introduced from the reference arm through the fiber coupler and is used for a guide maker on the sample surface in order to make it easy to aim the same target position to be measured. The spectral interference signal is converted to voltage, and digitized by a 14-bit DAQ board (PDA14, Signatec.Inc.) with sampling rate of $100 \mathrm{Msample} / \mathrm{sec}$ that is mounted inside a PC unit. The overview of the integrated SS-OCT system in the special rack is shown in Fig.5.2.

\section{Algorithm}

Algorithm of OCT program is shown in Fig. 5.5.

The start trigger or A-trigger for A-scan synchronization is fed from the integrated start trigger output from the source into the A/D converter. Each start trigger initiates the segmented sampling of 2048 counts that are transported to the PC, followed by fast Fourier transform (FFT) calculation. The start trigger is set to lag about $5 \%$ of total scan range after the actual rise of the lasing start in order to maintain stable detection. Because of this, pre-trigger mode is used in order to make use of $100 \%$ sampling over the effective scan range. Pre-trigger mode is a function in the DAQ board to recall the sampled data prior to the input start trigger using internal buffer on the board. Wavelength rescaling of interference signal is processed in the following steps. First, we obtain an interferogram with using a reflective mirror positioned at $1.5 \mathrm{~mm}$ depth position in order to extract the 
calibration data, i.e. the time array of peaks and valleys of the interferogram. This time array is then polynominally approximated up to $4^{\text {th }}$ order. By using this data set to rescale the sampled data in actual OCT measurement, the nonlinearity of the temporal frequency change can be properly compensated [41]. Signal processing is performed with using LabVIEW program. $512 \times 1024$ pixel image can be output within $0.3 \mathrm{sec}$ including process time for wavelength rescaling. The process time without wavelength rescaling was less than $0.1 \mathrm{sec}$, however, with LabVIEW-based program (Fig.5.6) we cannot accurately estimate the duration of process time because the other programs are running simultaneously consuming $\mathrm{PC}$ 's memory and run time. We expect the reduction of process time is significant when this is adapted to a programmed FPGA board.

The sensitivity of this SS-OCT is over $100 \mathrm{~dB}$ while the shot-noise limited sensitivity is 119 dB.

To realize a three-dimensional tomography such as an image shown in Fig.5.3, the galvano mirror for the B-scan is driven by a triangular wave. A B-scan trigger, which is synchronously generated for each B-scan, is used as an update clock of incremental position change in $\mathrm{C}$-scan axis, and the $\mathrm{C}$-scan trigger initiates spectral detection. Complete spectral signals and A-triggers for a single $\mathrm{C}$-scan are stored in the on-board memory of the A/D board, then transferred to the main memory of PC after the measurement, and finally stored on a hard disk. The stored data is loaded during the post-processing process, and arranged into a three-dimensional spectral signal by using the A-scan triggers; this is followed by the application of the above-mentioned signal processing.

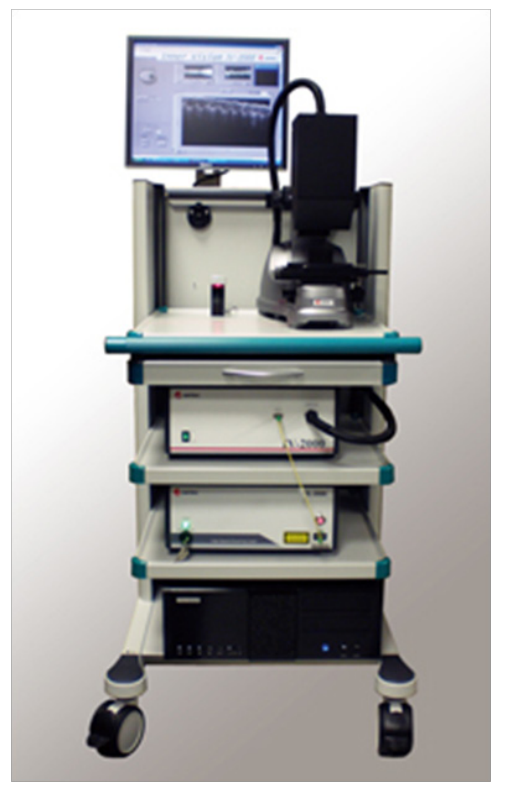

Figure 5.2 Rack-mounted SS-OCT system

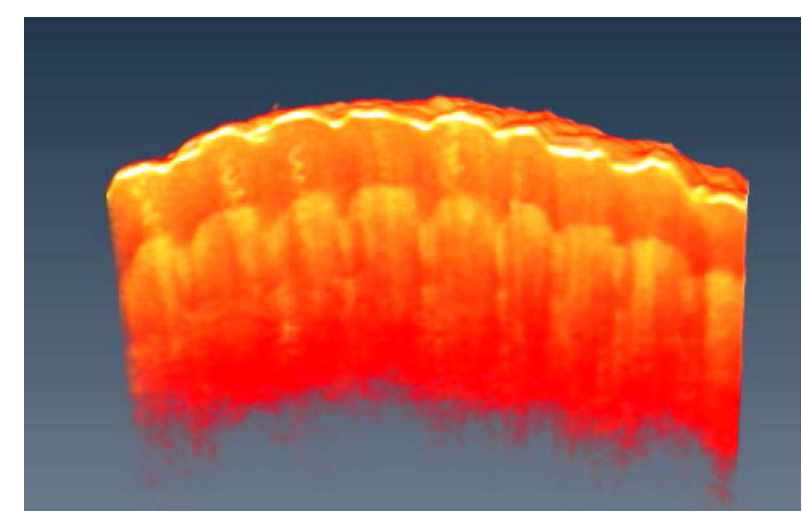

Figure 5.3 3D OCT image of human skin (finger) 


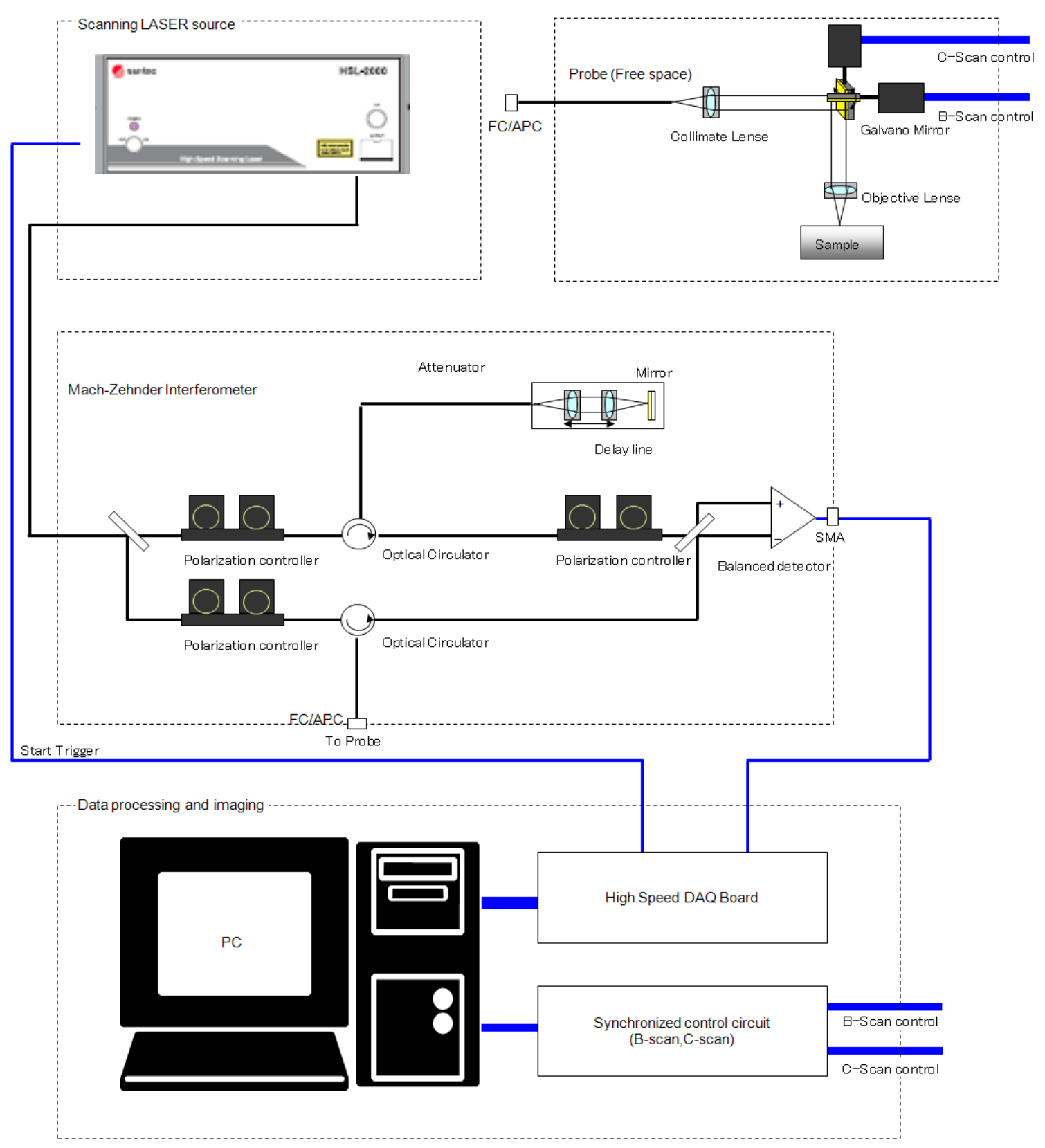

Figure 5.4 Hardware configuration 


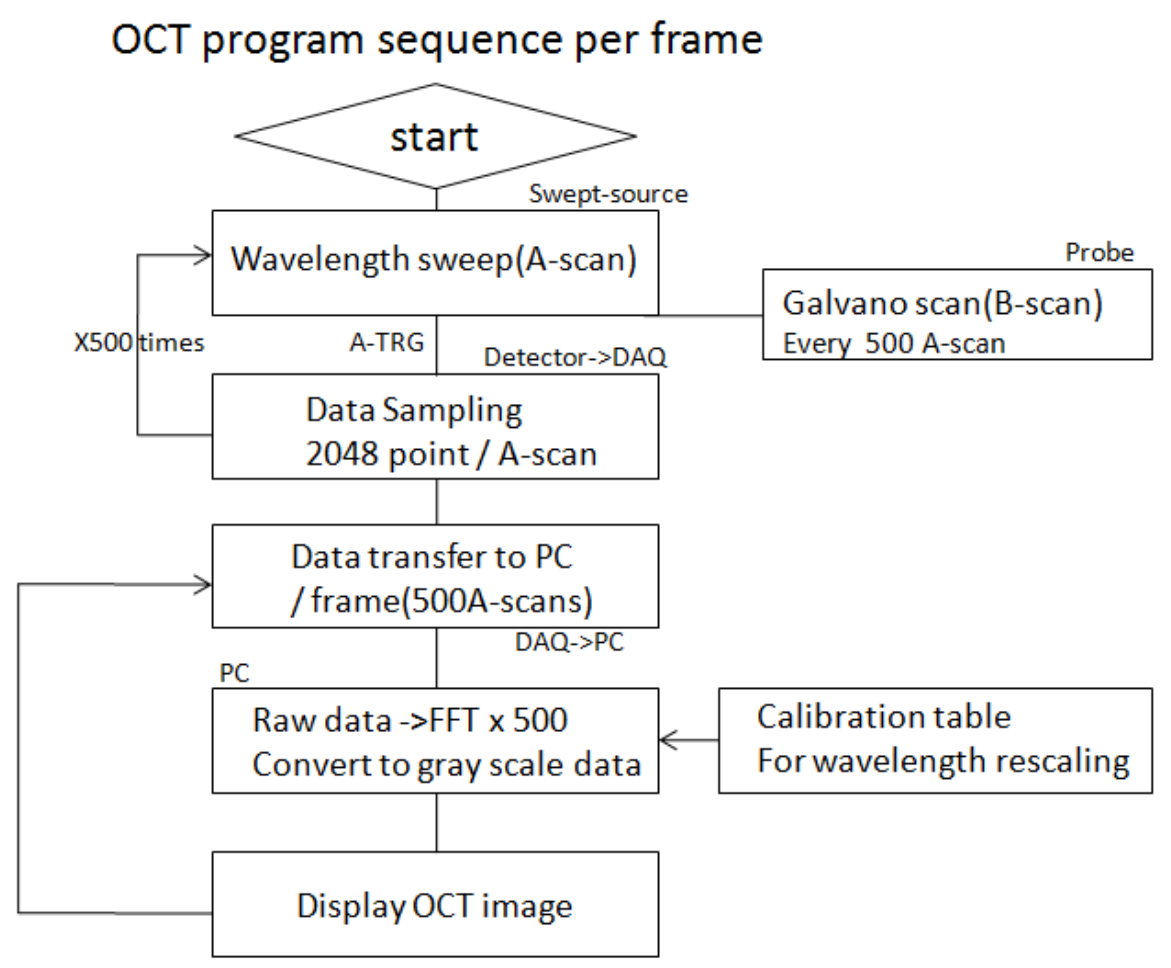

OCT program sequence per 3D volume

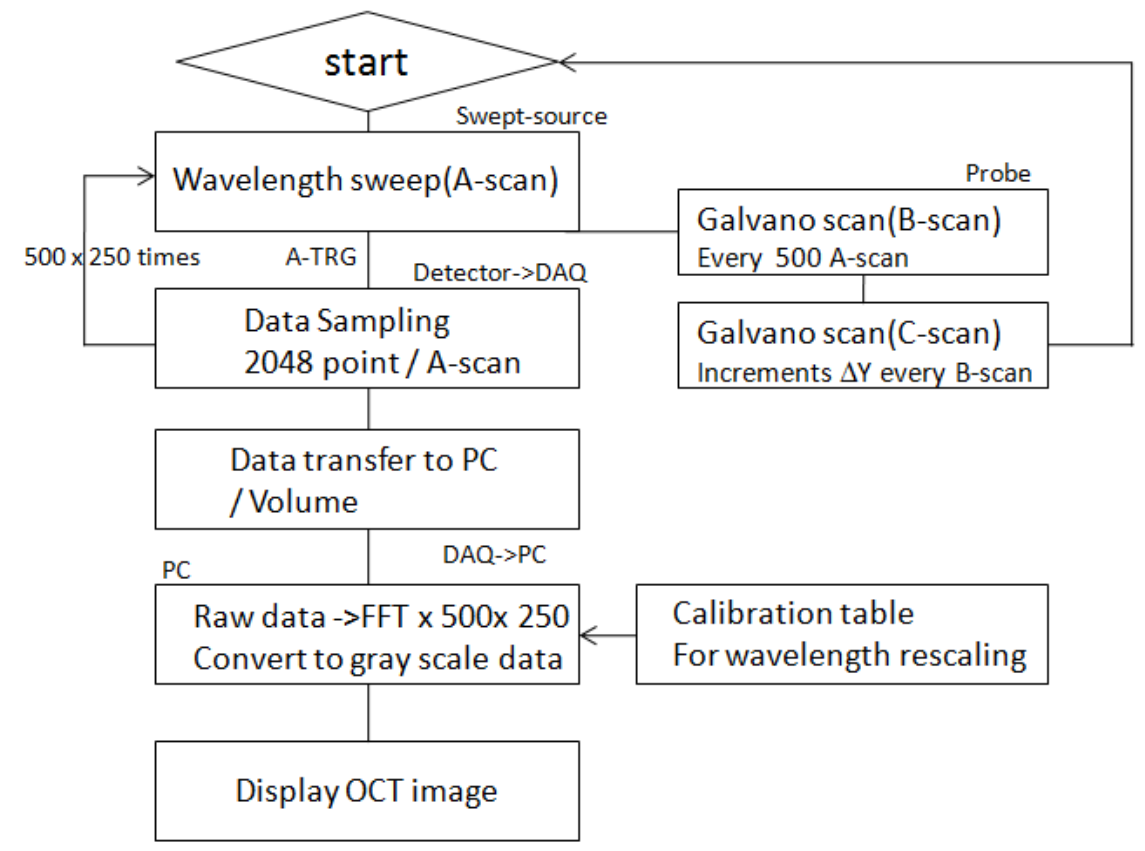

Figure5.5 SS-OCT program sequence 


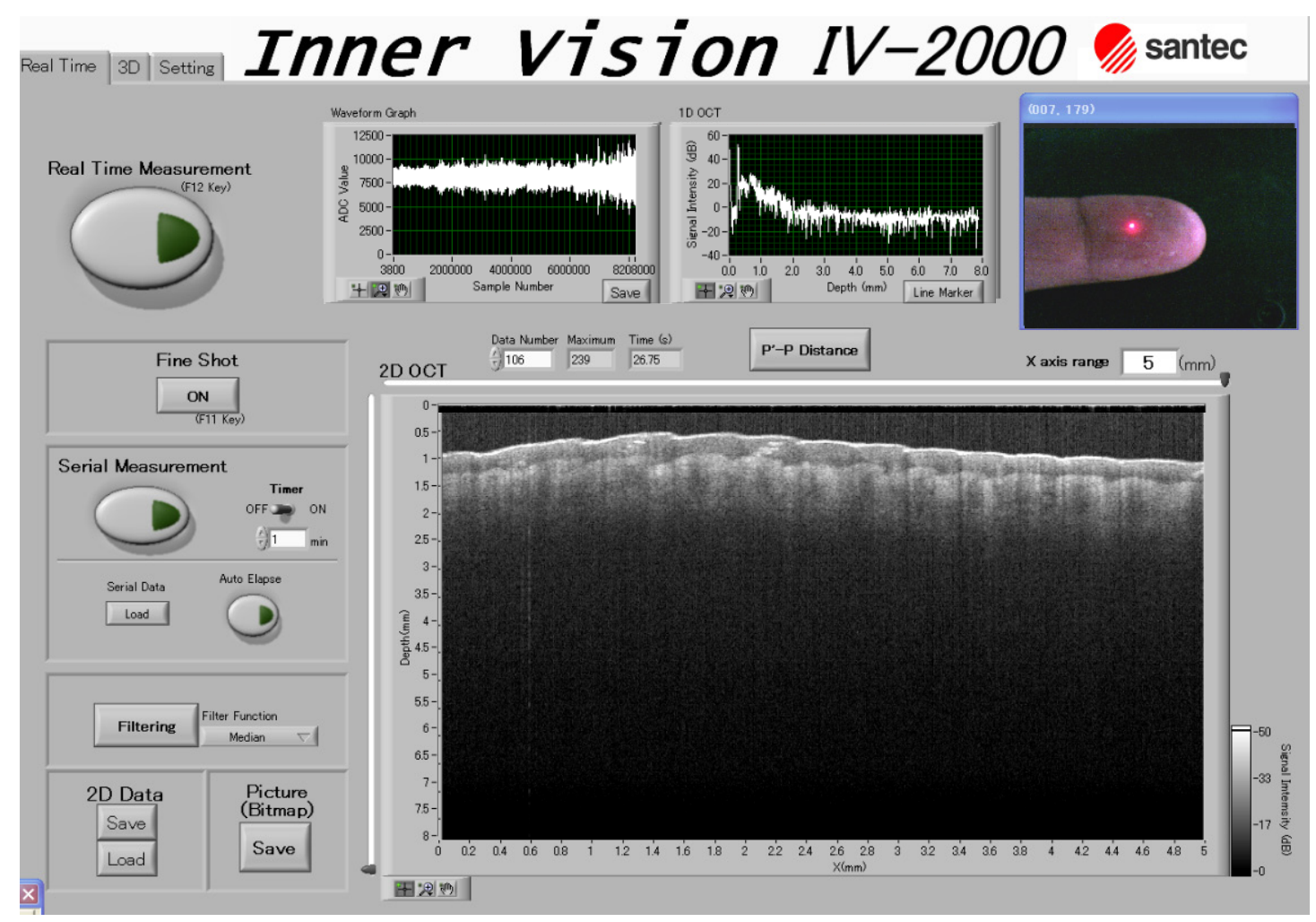

Figure 5.6 Example of SS-OCT system display

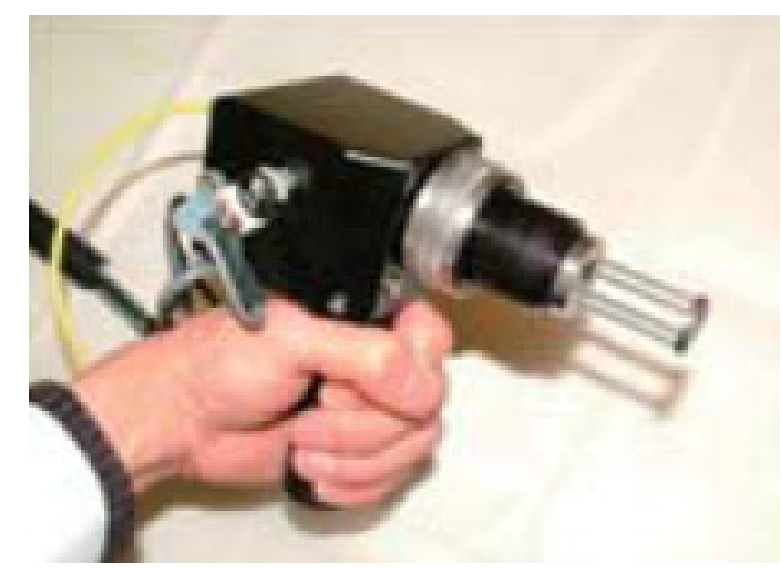

Figure 5.7 Photograph of a developed handheld probe 


\subsection{OCT image examples}

Unless otherwise noted, $1.3 \mu \mathrm{m}$ swept source with $120 \mathrm{~nm}$ swept range at $20 \mathrm{kHz}$ A-line rate is used for SS-OCT imaging in following examples. Coherence length of the source was $6 \mathrm{~mm}$.

A. Oral tissues

This work was done in collaboration with National Gerontology Center in Ohubu,-Shi, Japan. Oral tissue such as the structure of tooth can be imaged in cross-section by SS-OCT system using a compact handheld type probe as shown in Fig. 5.7. Clinical interest includes caries detection, inspection of the filling, and diagnosis of periodontal diseases as well as oral cancers [87-91]. Figure 5.8 shows an OCT image of a bottom-front tooth in comparison with image by X-ray. In OCT measurement, the beam from the probe is projected sideway with using a handheld probe shown in Fig. 5.2.7. Lateral resolution in this case was $15 \mu \mathrm{m}$. $\mathrm{X}$-ray image doesn't give three-dimensional information and its resolution is limited to $0.1 \mathrm{~mm}$ range. While, OCT image in cross-section reveals clear structure of tooth and gum, inside of which you can confirm the boarder of enamel and dentin junction and alveolar bone (yellow arrow). Observation of loss of alveolar bone with OCT can diagnose the periodontitis that may be able to replace the current method of physical probing to check the perio-pocket depth.
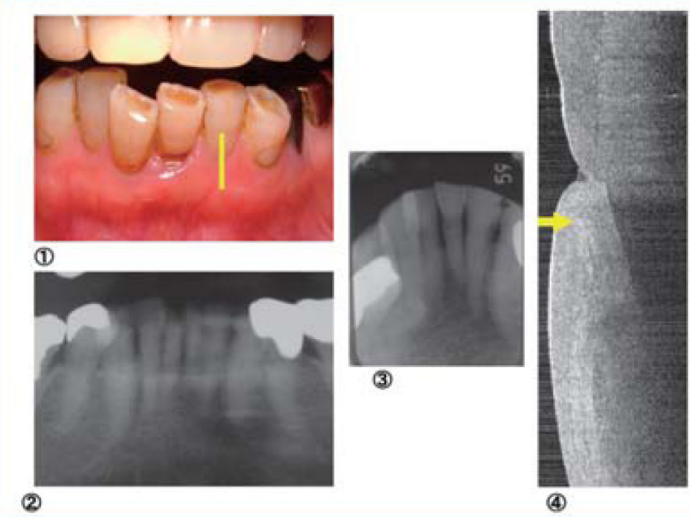

Figure 5.8 Comparison of X-ray and OCT image of the tooth

(1) photo of front teeth, (2) X-ray image from the side (3) $\mathrm{X}$ ray image from the top, OCT image (cross-section) in yellowed line in (1) of the tooth\#3 on the bottom)
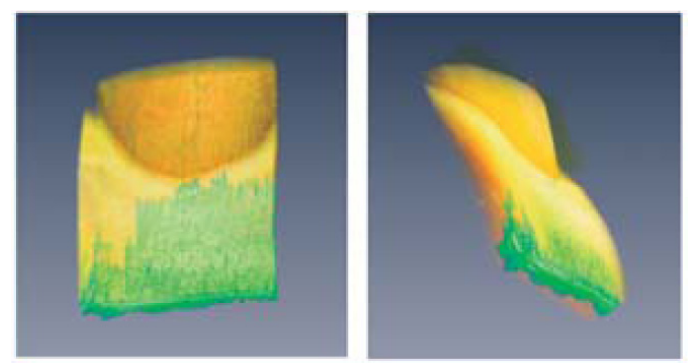

Figure 5.9 3D image of tooth with emphasized alveolar bone 
Figure 5.9 shows extracted and 3D-rendered OCT image of the tooth and gum with internal alveolar bone emphasized.

Figure 5.10 shows another example of a tooth that has resin filling near the gum. Left image shows very clear boundary of resin and enamel region and right image at tooth discovered a sign of decay inside the resin creating a bubble between the resin and dentin.

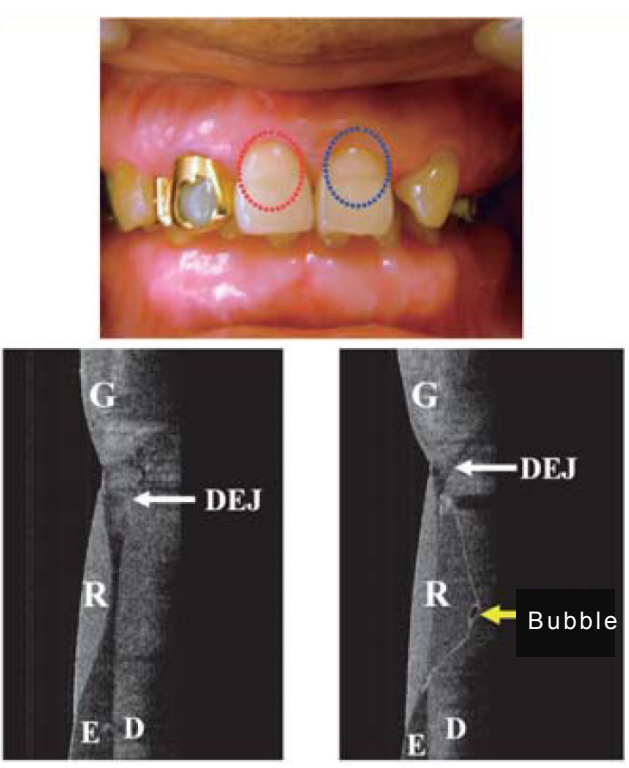

Figure 5.10. Resin filling

Figure 5.11 shows another 3-D OCT image of caries with C3 level, which means highly progressed caries, dematerializing the porous structure of the enamel and dentin. 3D image can be manipulated to show the structure in any arbitrary angle, slice, or perspectives when using a commercial 3D viewer program.
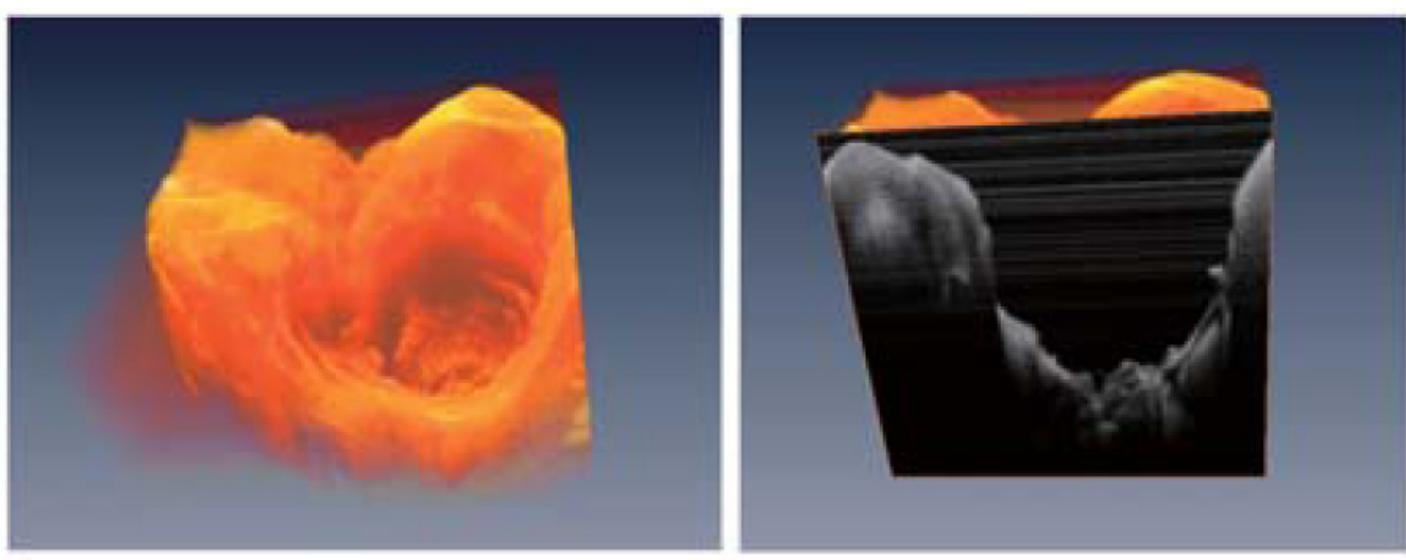

Figure 5.11 3D OCT image of caries

Figure 5.12 shows a capillary-venous malformation of the buccal mucosa; a type of oral tumor. The biopsy or pathological diagnosis of oral cancer is impossible because of danger 
of spreading the cancerous cells through the mouth to internal organs when extracting the sample. Hence, the OCT makes great advantage when it is used especially for oral tissues. Figure 5.13 shows 3D image of human Labial glands, which is, best to our knowledge, observed in real 3D tomograph for the first time. Observation of Labial glands provides with interesting aspect when diagnosing so called, "dry-mouth" symptom.
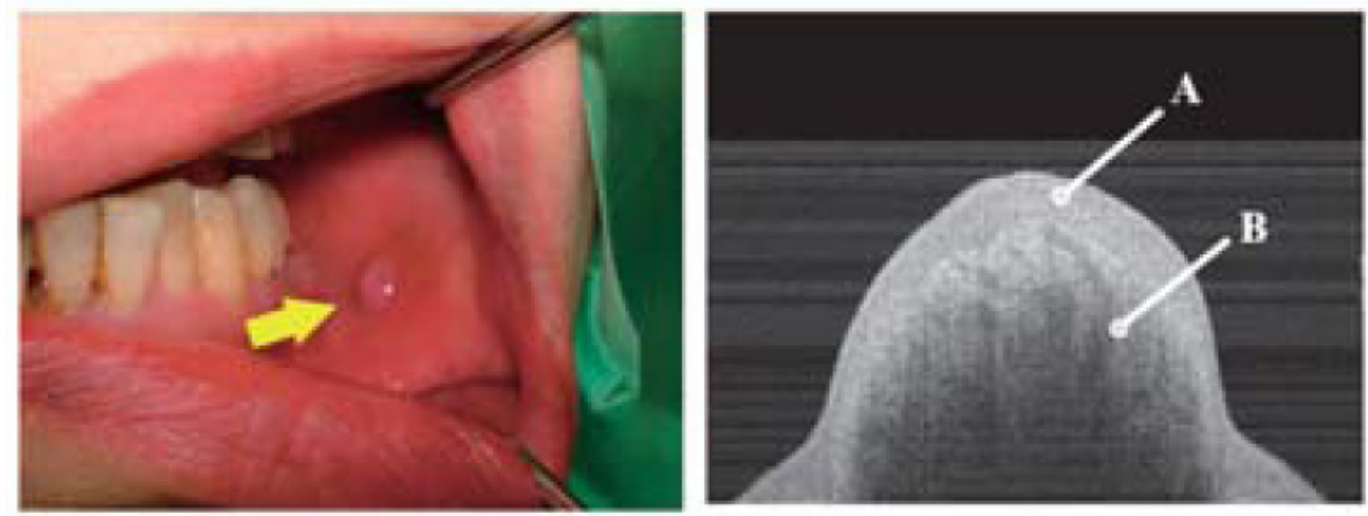

Figure 5.12 OCT image of a capillary-venous malformation of the buccal mucosa

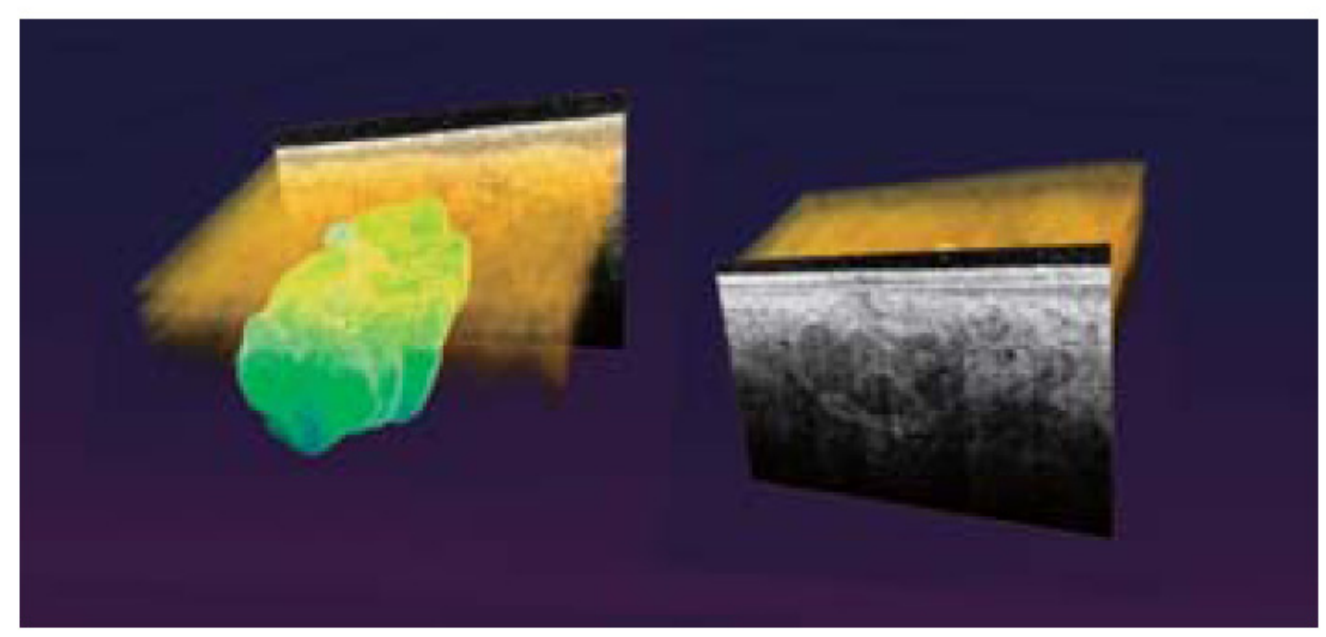

Figure 5.13 3D OCT image of human Labial glands 
B. Ophthalmology

(1) Anterior chamber imaging

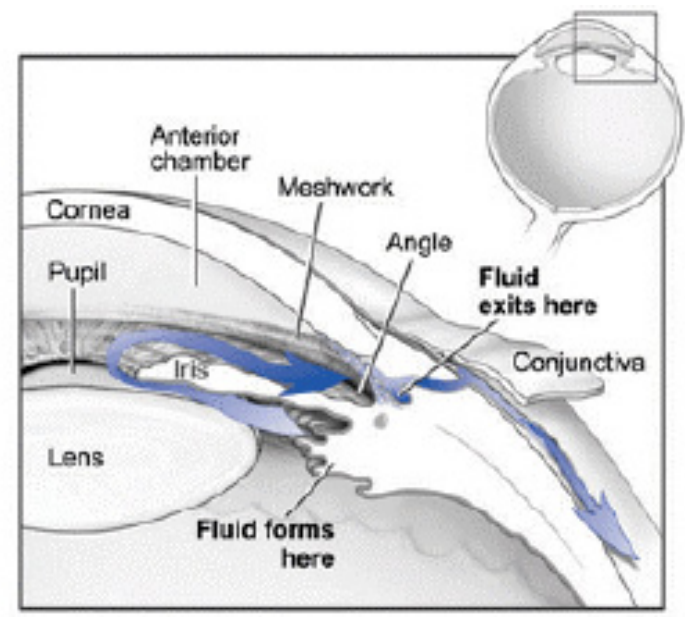

Figure 5.14 Diagram of Anterior chamber of human eye (courtesy of the National Eye Institute, National Institute of Health)

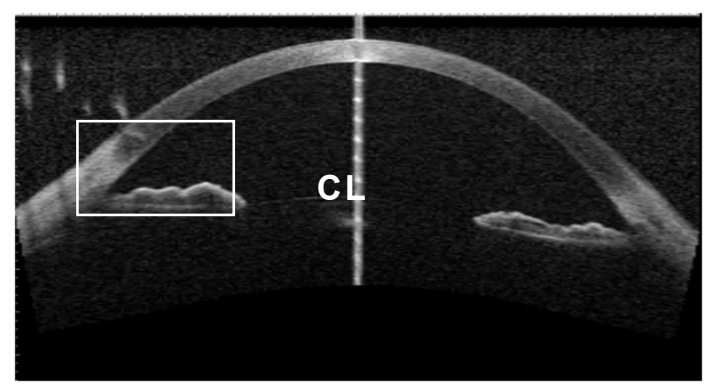

(a)

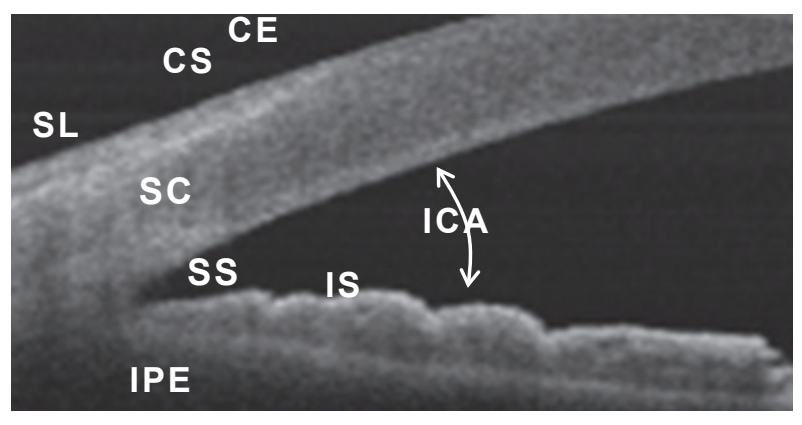

(b)

Figure 5.15 SS-OCT image of Anterior chamber of in-vivo human eye

Figures 5.15 (a), (b) show the tomographs of an in vivo human anterior eye segment. Several anatomic features are visible in (a) and (b) including sclera (SL), iris stroma (IS), iris pigment epithelium (IPE), crystalline lens (CL), scleral spur (SS), trabecular meshwork (TM),Schlemm's canal (SC). The corneal epithelium(CE) and corneal stroma (CS) are evident. This is the first time to visualize the full depth of anterior eye chamber, e.g., from the top of the cornea to bottom of the iris, by high-speed SS-OCT. The iridocorneal angle opening (ICA) and the curvature of the cornea are also evident in these figures. The probe power of these measurements was $2.9 \mathrm{~mW}$, which is well below the ANSI safety standard. 

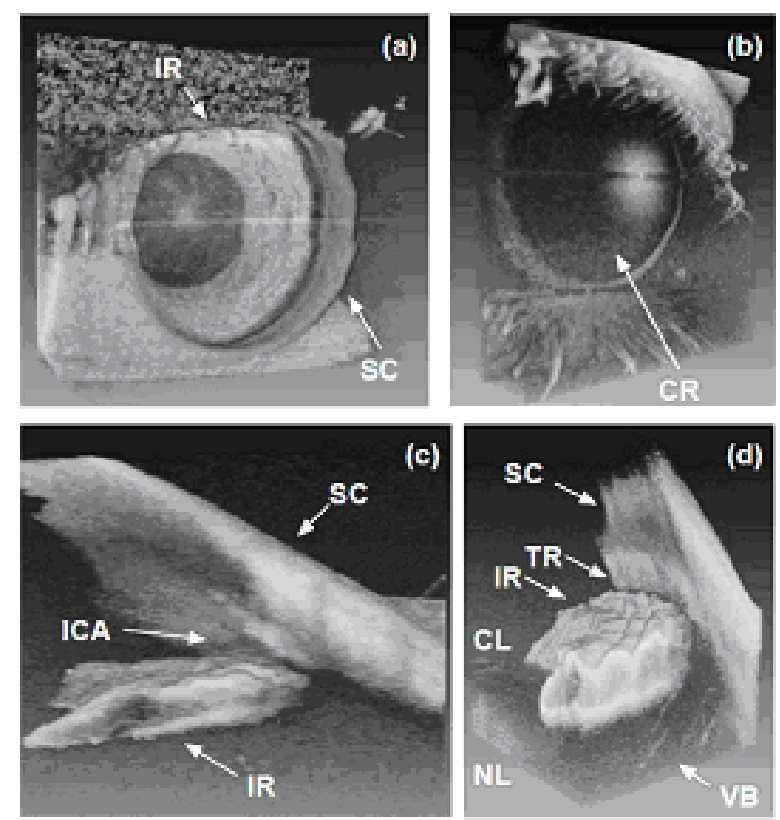

Figure 5.16 3D OCT image of anterior segment

Figure 5.16 shows three-dimensional tomographs of in vivo human anterior eye segments. (a) and (b) show the entire structures of the human iris (IR) and cornea, respectively. The measurement ranges are $16.5 \mathrm{~mm}$ (horizontal), $16.5 \mathrm{~mm}$ (vertical), and $6.0 \mathrm{~mm}$ (depth). (c) reveals the iridocorneal angle opening. In (d), the iridocorneal angle opening, crystalline lens, and nucleus lentis (NL) are evident. Trabecular meshwork (TM) and zonules of Zinn (ZZ), which are difficult to recognize in a two-dimensional OCT image, can be observed. The measurement ranges of (c) and (d) are $6.1 \mathrm{~mm}$ (horizontal), $6.1 \mathrm{~mm}$ (vertical), and 6.0 $\mathrm{mm}$ (depth) 


\subsection{Optically-actuated MEMS Scanning Fiber Probe for Medical Imaging}

This section presents a novel configuration of MEMS scanning endoscope that is actuated by external optical modulation. Light at a wavelength in the $1550 \mathrm{~nm}$ range is used to modulate a scanning MEMS mirror. The instrument also provides for the delivery of a second beam at a wavelength of $1310 \mathrm{~nm}$, commonly used for medical diagnostic application such as optical coherence tomography (OCT). The two wavelengths are combined with using a WDM filter and launched into a single mode fiber. This novel approach provides for the operation of a scanning endoscope without the need for directly powering up and wiring to the scanning element, in this way a less hazardous apparatus for in-vivo diagnosis is possible as well as compact size. Design of the module and MEMS scanning mirror is discussed. A fabricated module is demonstrated in which a MEMS mirror scans at the resonant frequency of $300 \mathrm{~Hz}$ generating a optical scanning angle of $8 \mathrm{degrees}$.

\section{Catheter or fiber probe for endoscopic OCT applications}

The scanning fiber-optic probe/catheter technique has increased the potential of endoscopic medical applications. Optical coherence tomography is one of such applications that require the beam scanning inside the organs such as when diagnosing a gastrointestinal tact or monitoring plaque in the vascular vein $[11,40,92]$. Scanned line or area is visualized as a cross section or a 3-dimensional volume image for diagnosis imaging. Conventional way to scan the beam across the subject tissue area is to simply rotate or translate the fiber mechanically by manipulating from outside of the body as shown in Fig. 5.17. Either pulling the fiber out or rotating the fiber by using the rotary joint makes translation or rotation of the probe possible [40].

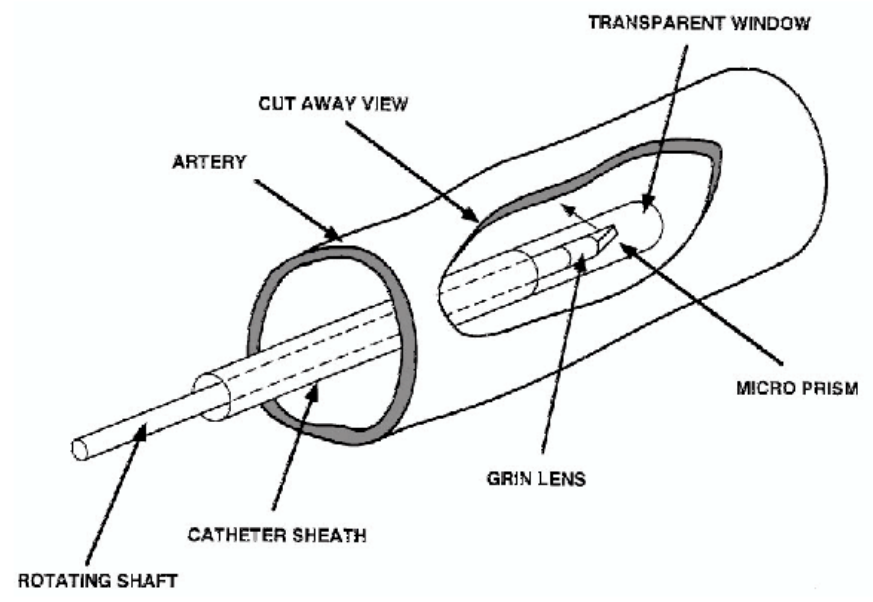

Figure 5.17. Rotary type scanning catheter for OCT (ref [40]) 
However, such technique always involves concerns on accidents of breakage of the fiber due to excessive stress during operation of the fiber probe. Amongst the various technologies that facilitate the scanning of an optical beam for use in endoscopic procedures it is MEMS-base catheters that have many advantages in size, speed, accuracy, and repeatability over the conventional scanning techniques that requires the whole fiber endoscope to be mechanically rotated or translated with respect to the tissue [93,94].

Previously, for endoscopic OCT, MEMS scanning mirrors have typically required a relatively higher voltage supply in order to operate. The high voltage supply is required to deflect the electrostatic MEMS mirror to a sufficiently large angle to cover a wide imaging area. However, high voltage electricity is hazardous for in vivo diagnosis since the wire along the fiber to the endoscope tip can be accidentally exposed to the intralipid or blood that can result in electrocution. In order to avoid this kind of accident, whole fiber probe should be perfectly sealed so that any liquid immerses inside the housing and the wire. This protection may increase the size and the radius of the fiber probe. And modulation of electricity itself may even create electro-magnetic interference (EMI) to the other medical apparatus during the operation.
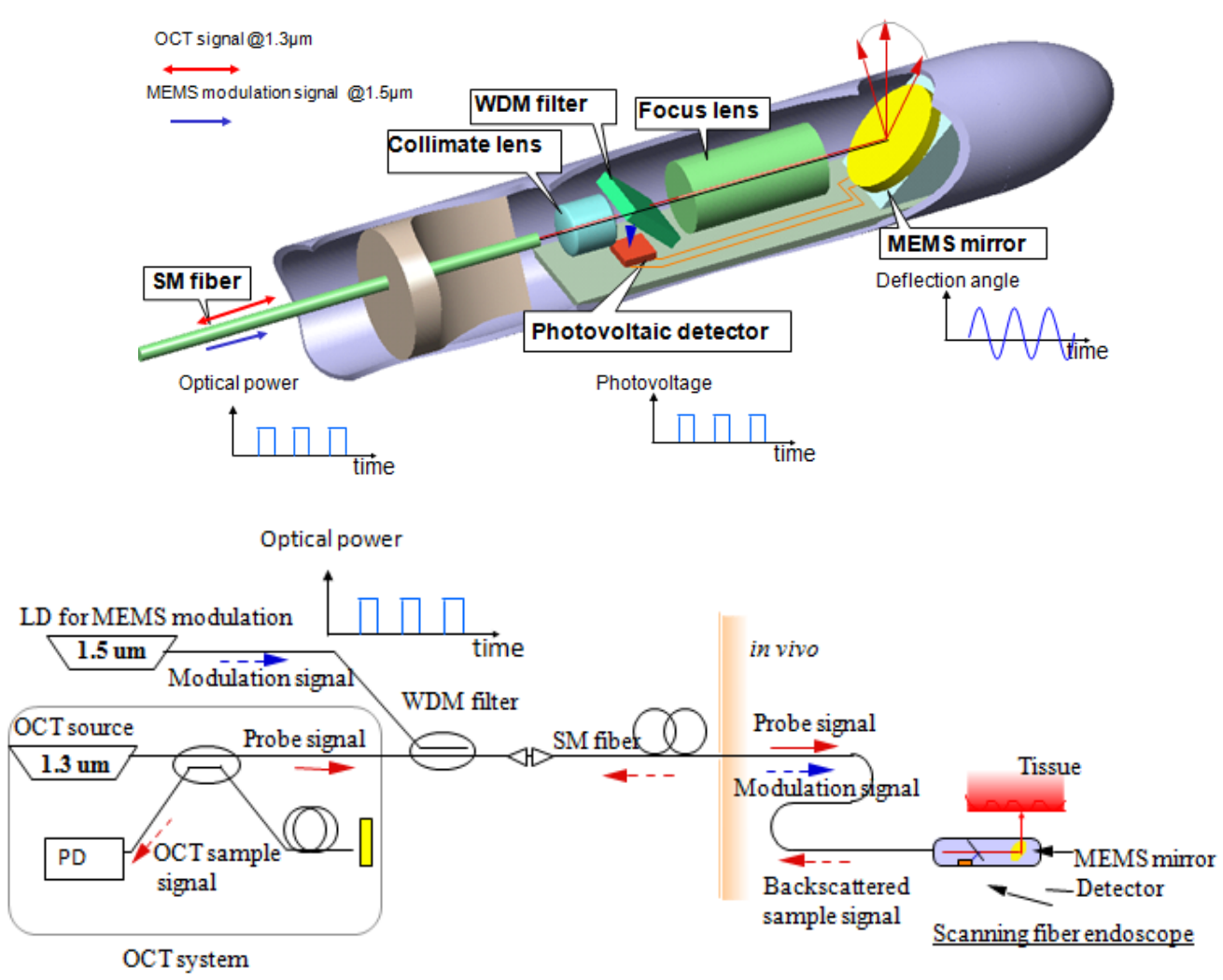

Figure 5.18 Schematic diagram of optically modulated MEMS probe (Top) and OCT system configuration with the probe (Bottom) 
In this section 5.4, an endoscope with a low voltage-driven MEMS scanner and photovoltaic detector is proposed,. Modulation of the MEMS scanning mirror is achieved using an optical signal that is introduced from outside of the body, thereby negating the need for electrical supply and wiring within the endoscope and eliminating EMI. MEMS scanner is designed to operate at resonant frequency of the device so that relatively higher scanning angle can be achieved. Photovoltaic detector with relatively higher response speed is used for conversion of optical signal into drive voltage for MEMS actuation. Multiplexing with a WDM bandpass filter inside the fiber probe accommodates different wavelengths respectively for OCT signal and MEMS actuation. This application is not just limited to the use in OCT but also suitable for the endoscopic confocal microscopy, or fluorescence spectroscopy as long as the wavelength range of the interest in the measurement is different from the optical modulation signal for MEMS actuation.

\section{Concept of optically modulated MEMS scanning endoscope}

The proposed MEMS scanning endoscope consists of a GRIN lens, a WDM bandpass filter, a photovoltaic detector, and a MEMS scanning mirror packaged in a cylindrical tube as shown in Fig. 5.18. The Photovoltaic detector and MEMS mirror are mounted on a ceramic submount and connected electrically. Two light signals at different wavelength ranges are combined with a WDM combiner filter, launched into a single mode fiber and transmitted to the endoscope. The two signals are; incoming optical probe light from an OCT light source at $1310 \mathrm{~nm}$ and the externally coupled modulation signal at $1550 \mathrm{~nm}$. After collimation by a GRIN lens, the modulation signal at $1550 \mathrm{~nm}$ is reflected by a WDM bandpass filter to the photovoltaic detector and converted to voltage that activates the MEMS scanning mirror. The probe light at $1310 \mathrm{~nm}$ passes through the WDM filter and propagates through a focusing lens to the MEMS scanning mirror.

The WDM filter in this case is a low pass edge filter type, having 1260-1360 nm passband and $1550 \mathrm{~nm}$ reflection band with isolation of over $30 \mathrm{~dB}$ in 20 to $45 \mathrm{degree}$ tilt with respect to the optical axis. After transmission through the WDM filter, a lens of $3.26 \mathrm{~mm}$ focal length focuses the collimated $1310 \mathrm{~nm}$ signal. The Gaussian beam size before focusing is $400 \mu \mathrm{m}$, the focused spot size is $20 \mu \mathrm{m}$. The working distance of the lens $(3.26 \mathrm{~mm})$ was chosen to focus the beam at the endoscope tube circumference (5 $\mathrm{mm}$ diameter) after deflection by the MEMS mirror, in this way the endoscope is optimized for inspection of samples in touch with the observation window. Here, Pyrex capillary tube is used for the package. The MEMS mirror is placed right after the lens to reflect the light to the direction perpendicular to the optical axis. When actuated the MEMS mirror scans the $1310 \mathrm{~nm}$ beam in the radial direction 
with respect to z-axis in order to image the cross sectional of cylindrical organs such as veins or gastro intestines using the OCT technique. If the mirror is place in 45 degree tilt, optical scanning angle is 1.4 times the mechanical tilt of the mirror in the case of radial scanning. In this study the target optical scanning angle was set to 10 degree, so the required mechanical tilt of MEMS mirror is about 7 degree. In order to efficiently convert the photovoltage to a large mechanical scanning angle, MEMS mirror is designed to operate in a resonant mode at a constant frequency corresponding to the image-acquisition rate. Appropriate selection of two lenses can be used to adjust the working distance and focal spot size for the different applications. Outside the body, external laser diode that is directly modulated by the injection current is introduced at the probe arm part of the system, so the application of this fiber probe doesn't limit the type of system as long as different wavelength bands are used for the system and modulation signal.

In the case of OCT application as shown in Fig.5.18(bottom), backscattered light from the sample carrying 1-dimensional depth information is coupled back to the same optical path going through the WDM filter and travels back via single mode fiber toward the OCT system for analysis. Analysis is sequentially carried out over the scanned area to construct the 2-dimensional radial cross section of the sample in results after an appropriate signal processing.

The module design is completely self-contained so that it is easy to make the package sealed at the tip of the fiber probe and the rest is just a single mode fiber along.

Table 5.1 MEMS target specifications

\begin{tabular}{|l|l|l|}
\hline & Specification & Note \\
\hline Item & $1(\mathrm{x}) \mathrm{x} 2(\mathrm{y}) \mathrm{mm}$ & Rectangular \\
\hline Scanning angle & Single $\mathrm{x}$-axis & \\
\hline Scanning rate & $300 \mathrm{~Hz}$ & Resonant mode \\
\hline Scanning angle & $10 \mathrm{deg}$ (optical) & 7 deg (mechanical) \\
\hline
\end{tabular}


MEMS mirror

The MEMS scanning mirror for this module is a single axis scanner and has angular vertical comb (AVC) actuators, that provide a large mechanical tilt angle for low voltage bias [95,96]. Figure 5.19 shows a photograph of the MEMS mirror. The device is made of SOI (30 $\mu \mathrm{m}$ silicon/ $4 \mu \mathrm{m}$ oxide/ $400 \mu \mathrm{m}$ silicon substrate).

Figure 5.20 shows the process flow of the MEMS scanner. AVC is formed in a single mask process on the device layer of an SOI wafer. Initial angle offset of movable comb tooth with respect to the stationary comb tooth is realized by so called self-assembly process utilizing in-process stiction in the drying step after the wet sacrificial etching of the membrane [97] as shown in Fig.5.21.

Substrate underneath the mirror is deep dry etched from the bottom so that it liberates the angle of scanning. The stiction pad buckles down to the handle wafer after the sacrificial etching process and is held permanently in place by molecular binding force. This bucking action of the pad introduces the twist of torsion on the hinge that is connected to the stiction pad, and a initial angular offset of the mirror is set as a result. Compared to the other techniques for introducing an offset angle in vertical comb actuators, such as those involving complicated two steps of photolithography and deep dry etching process, our approach only needs one mask for definition of AVC and thus is a robust process through which high device yields are possible. In our design, initial offset angle of about 2-3deg can be created in this design when using $4 \mu \mathrm{m}$ BOA layer. The MEMS mirror is designed to operate in resonant mode since the probe allowing it to repeatedly scan over a large scan angle at a constant frequency corresponding to the image-acquisition rate. . The size of comb is $150 \mu \mathrm{m}$ long, $10 \mu \mathrm{m}$ wide with a gap of $3 \mu \mathrm{m}$ in between combs. The number of comb $\mathrm{s}$ on the single side of the mirror is 58. The MEMS mirror has dimensions of $1 \mathrm{~mm}$ wide, $2 \mathrm{~mm}$ long in the direction parallel to the optical axis. MEMS mirror actuation is driven with a bias voltage between 0 to $+5 \mathrm{~V}$ at the resonant frequency of $300 \mathrm{~Hz}$. For applications such as OCT a slower rate, close to video rate, is desirable for lateral scanning of sample. The shape of MEMS chip is elliptically rounded so that it fits well into the cylindrical tubes at a 45 degree tilted angle for radial scanning. 


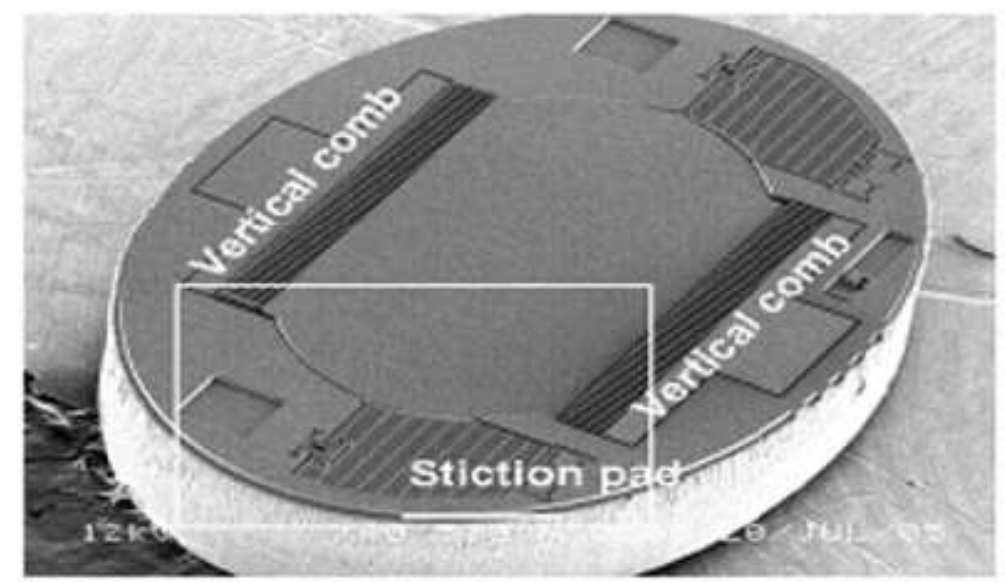

(a)

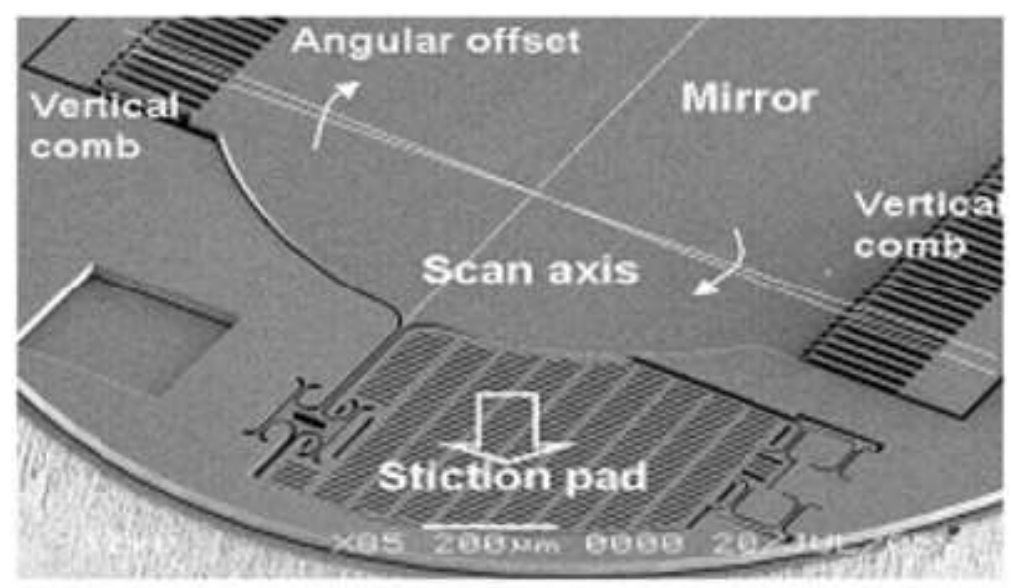

(b)

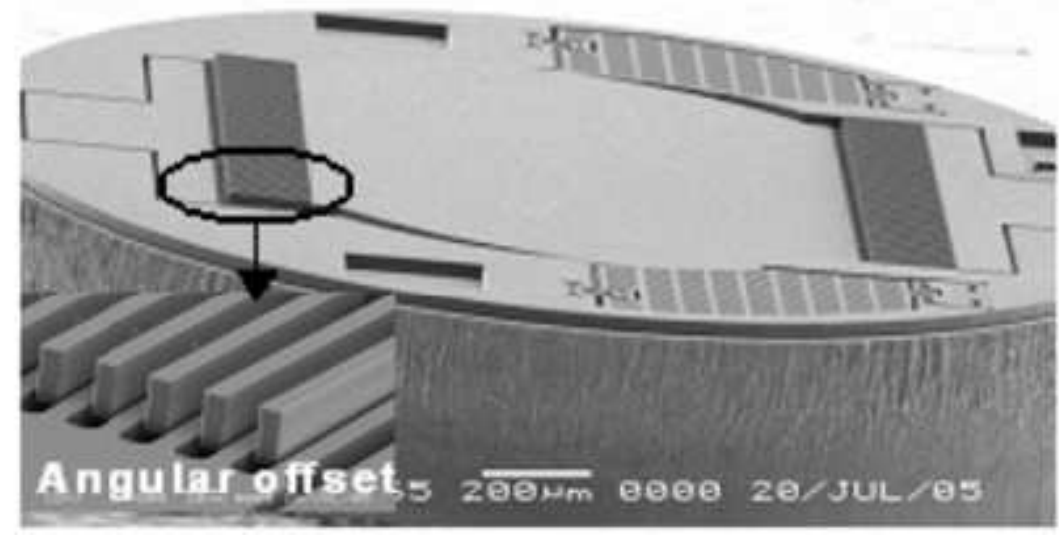

(c)

Figure 5.19 MEMS tilt mirror with angular vertical comb drive 


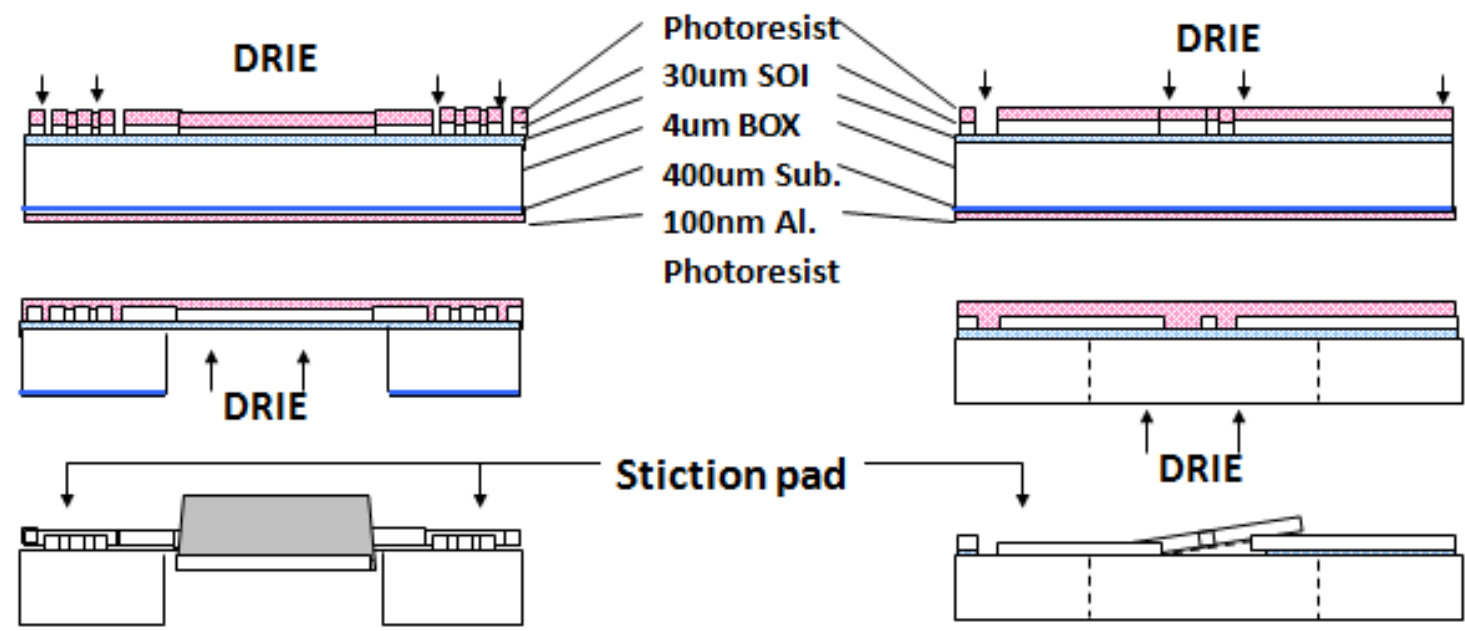

Side $\mathrm{X}$

Side $Y$

Figure 5.20. MEMS fabrication process step with bulk SOI wafer

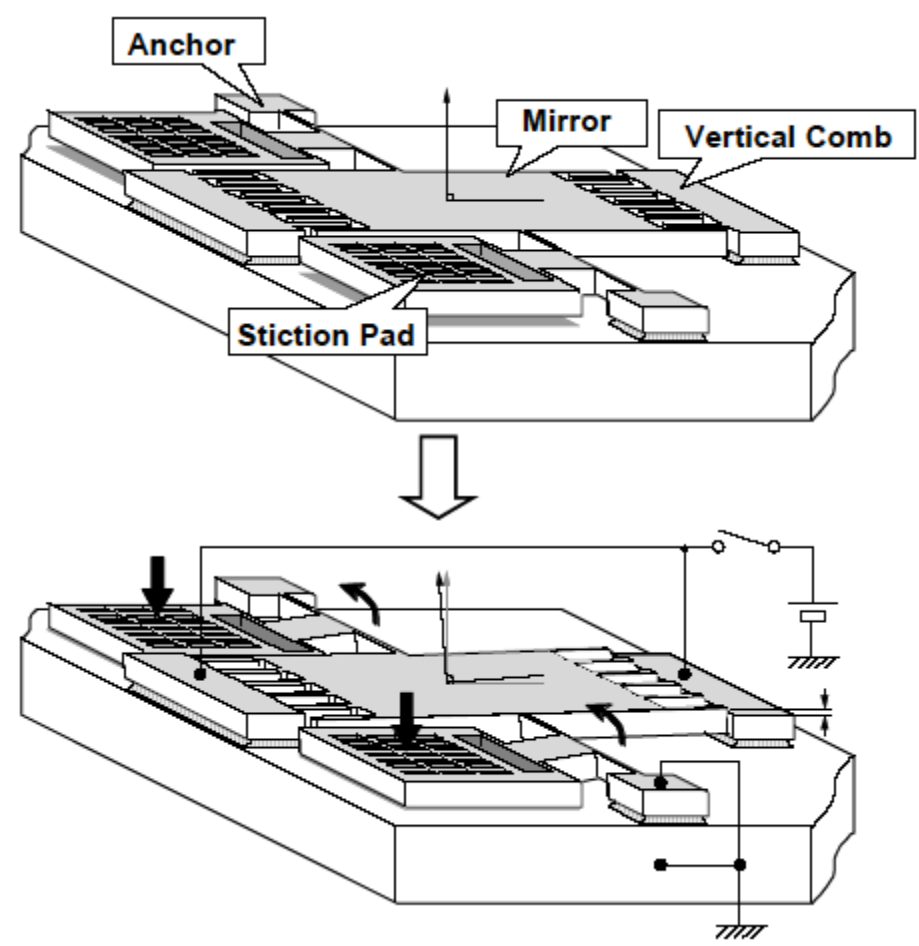

Figure 5.21 Mechanism of buckling of stiction pad 


\section{Photovoltaic detector}

The photovoltaic detector used in this study is based on an asymmetric d-doped superlattice structure is used (Fig.5.21 (a))[98,99]. Unlike the other photodetectors, this structure makes the device operate as voltage source and has large bandwidth. The device structure was originally proposed for the direct modulation of field effect transistor (FET) by photovoltage [100].

Figure 5.22 (b) shows the responsitivity of the detector. The solid line indicates the voltage generated by $1550 \mathrm{~nm}$ light focused onto the detector with spot size of $30-40 \mathrm{~mm}$, the dotted line shows the voltage generated by the detector when illuminated by a collimated beam of $400 \mu \mathrm{m}$ diameter. We found that although there is a mismatch between the $400 \mu \mathrm{m}$ collimated beam and the effective area of detector, the voltage generated is sufficient for operation of the MEMS mirror. So, in our configuration, the $1550 \mathrm{~nm}$ beam is directly projected onto the detector after collimation by the GRIN lens and reflection by the bandpass filter. A measured saturated voltage of $5 \mathrm{~V}$ is the highest open-circuit voltage generated by a single photovoltaic detector illuminated by $1550 \mathrm{~nm}$ light.

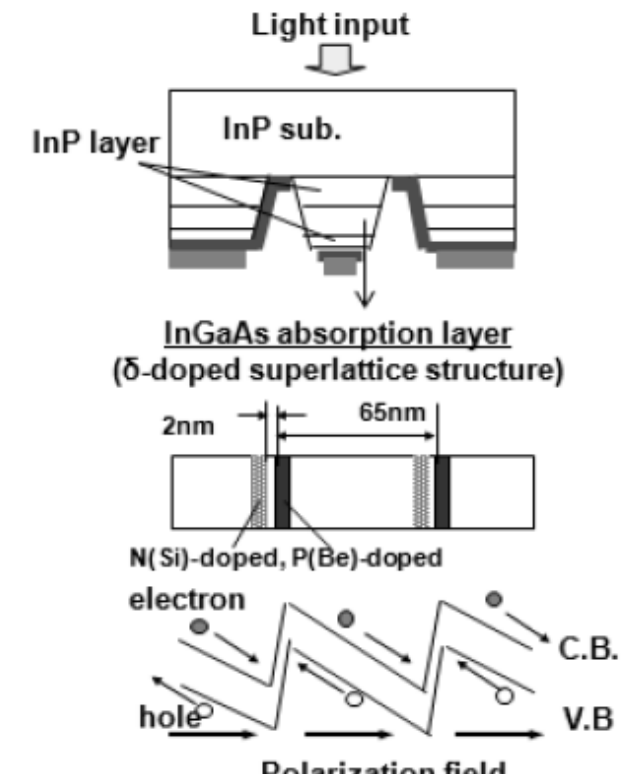

Polarization field

(a)

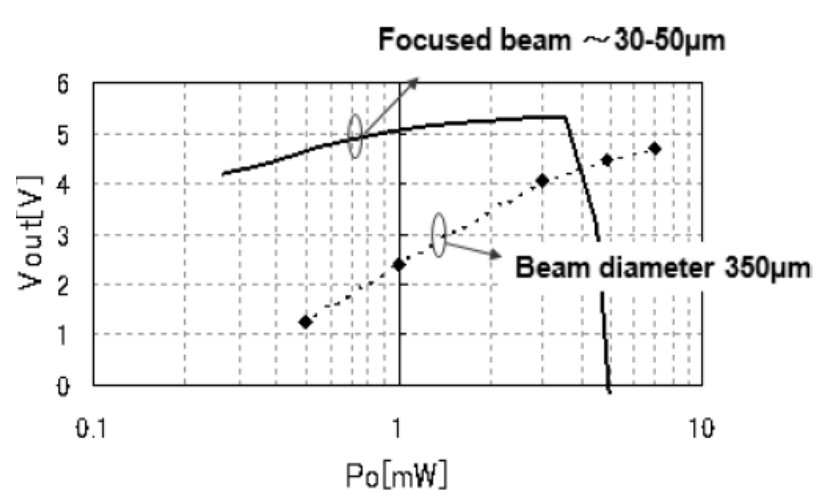

(b)

Figure 5.22. (a)Schematic diagram of photovoltaic cell and (b)generated voltage characteristics

Figure 5.23(a) and 5(b) show the response time of the detector at $60 \mathrm{~Hz}, 1 \mathrm{kHz}$ modulation, respectively. Illuminating laser is directly modulated by injection current. Input power has a extinction ratio of $>90 \%$ with $10 \mathrm{~mW}$ peak power when amplitude-modulated. In the case of $1 \mathrm{kHz}$ modulation, the extinction ratio was set to about $6 \mathrm{~dB}$ above the threshold level of the 


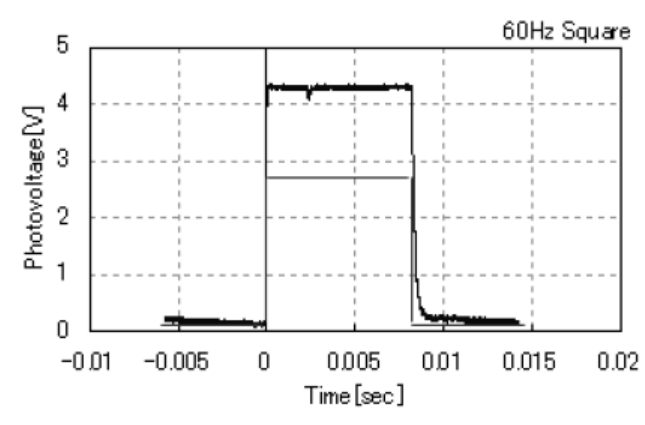

(a) $60 \mathrm{~Hz}$ square waveform

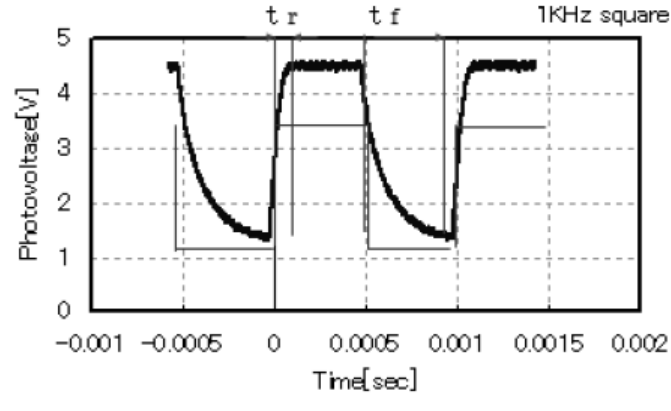

(b) $1 \mathrm{kHz}$ square waveform

Figure 5.23 Response of photovoltaic cell to the optical intensity modulation;

laser. At $60 \mathrm{~Hz}$, it can be seen that the generated photovoltage has square waveform almost identical to the input optical signal. As the modulation speed increases up to $1 \mathrm{kHz}$, fall-off tends to slower to the bottom. Rise time is about $50 \mu \mathrm{sec}$, and fall time is about $200 \mu \mathrm{sec}$, which is still sufficiently fast to achieve several hundreds $\mathrm{Hz}$ of scan rate for MEMS scanner. Intrinsic response speed of the device in principle is determined by dielectric relaxation time that is on the order of picoseconds.

The size of the chip is about $300 \times 500 \mu \mathrm{m}$ and the chip is flip-chip mounted on the ceramic submount. The light is illuminated from the bottom side.

\section{WDM bandpass filter}

WDM filter is a thin-film-base bandpass filter (edge filter type) with a wide transmission bandwidth for $1.3 \mathrm{um}$ range. A sample of filter was fabricated with ion beam assisted deposition method. Filter is aligned at $20 \mathrm{deg}$ to the optical axis so that polarization dependence of input signal and the back-reflection from the photovoltaic detector surface are both negligible. Figure 5.24 (top) shows the simulated filter profile of transmission and reflection at 20 degree incident angle, respectively. 1.3 um OCT signal window is flat over a large passband over $1260 \mathrm{~nm}$ to $1360 \mathrm{~nm}$ and has a high isolation of over $40 \mathrm{~dB}$ with respect to the $1.5 \mathrm{um}$ band. There is an addition of another $40 \mathrm{~dB}$ that comes from a combiner filter at the system outside. Total of $120 \mathrm{~dB}$ isolation adds up at the system side between $1310 \mathrm{~nm}$ and $1550 \mathrm{~nm}$ band. Thus, no significant cross talk from modulation signal concerns the OCT systems sensitivity.

As for photovoltage generation in reflected path, $1550 \mathrm{~nm}$ has $10 \mathrm{~dB}$ isolation to the $1310 \mathrm{~nm}$ band, which is relatively small compared to transmission path isolation. However, since the OCT probe power level at $1310 \mathrm{~nm}$ is usually lower than $1550 \mathrm{~nm}$ by the factor of over $3 \mathrm{~dB}$, total of about $13 \mathrm{~dB}$ is enough not to be influence by probe power for MEMS operation. 
Figure 5.24 (bottom)shows the measured spectrum of WDM filter. Polarization dependent loss is less than $0.1 \mathrm{~dB}$ for both bands.
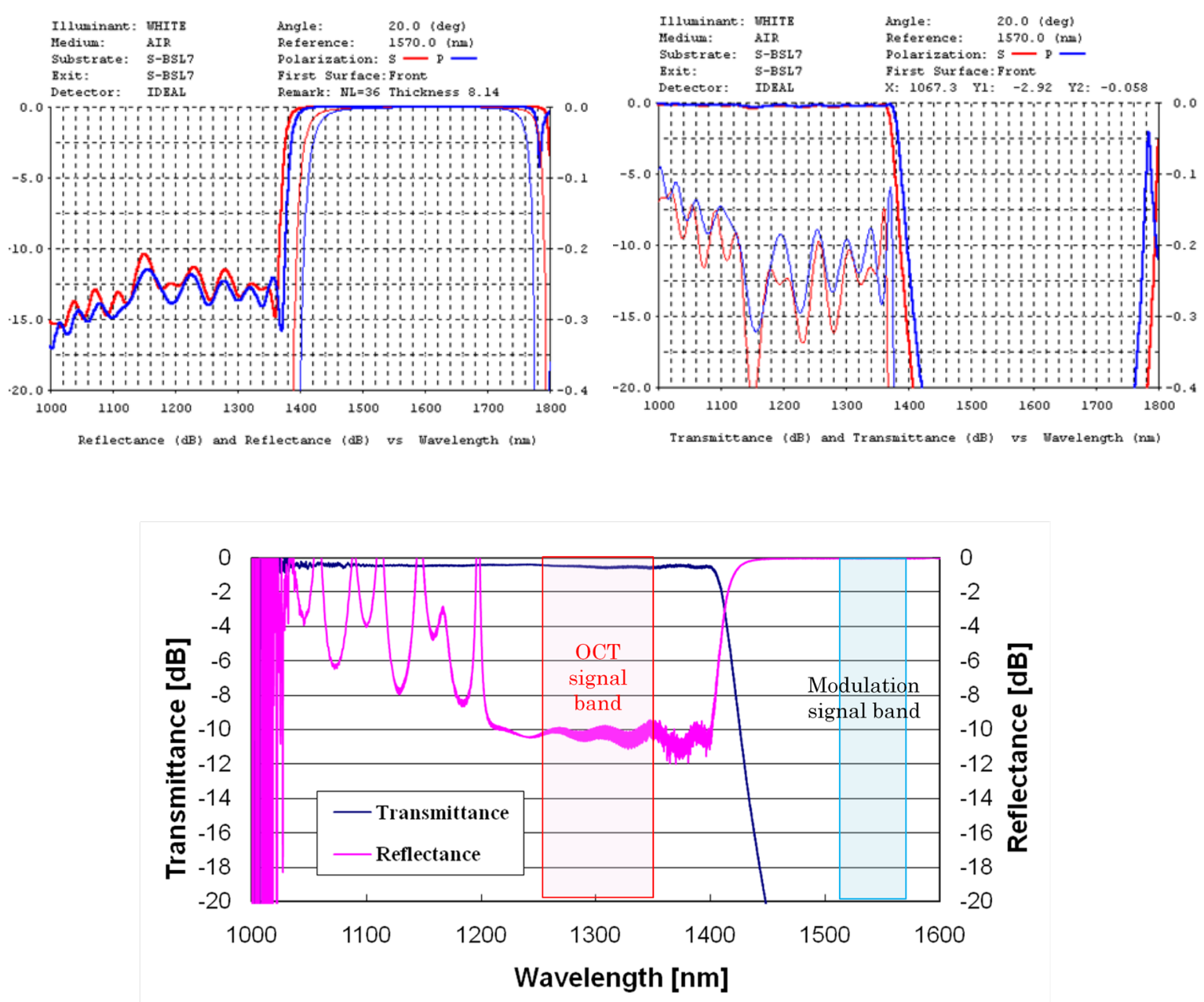

Figure 5.24 Bandpass filter characteristics: Reflection spectra (left), Transmission spectra (right) Measured spectrum with OCT probe signal band and modulation signal band (bottom) 
Results

Figure 5.25(right) shows the resonant frequency of MEMS scanning mirror measured by laser Doppler velocimeter. The first order resonance was found at frequency of around $300 \mathrm{~Hz}$. Figure 5.25 (left) shows the tilt angle of the MEMS mirror versus applied voltage in DC mode. By operating the mirror in resonance it is possible to amplify the DC tilt angle of about 0.45 degree at $5 \mathrm{~V}$ DC bias by a gain factor of over $15 \mathrm{X}$. Next, basic operation by optical modulation was confirmed. The MEMS scanning mirror is connected to the photovoltaic detector and light at $1550 \mathrm{~nm}$ is projected onto the detector. The $1550 \mathrm{~nm}$ laser diode output power used to illuminate the detector is directly modulated between $0 \mathrm{~mW}$ and $7 \mathrm{~mW}$ by injection current modulation at $300 \mathrm{~Hz}$ using a function generator in both TTL square waveform and sinusoidal waveform modes. Scanning angle of the MEMS mirror was measured by projecting a red visible diode laser beam at $633 \mathrm{~nm}$ on the mirror as shown in Fig. 5.26. By tracing the length of line beam created by scanning the mirror the optical scan angle was found to be 8 -10degrees.
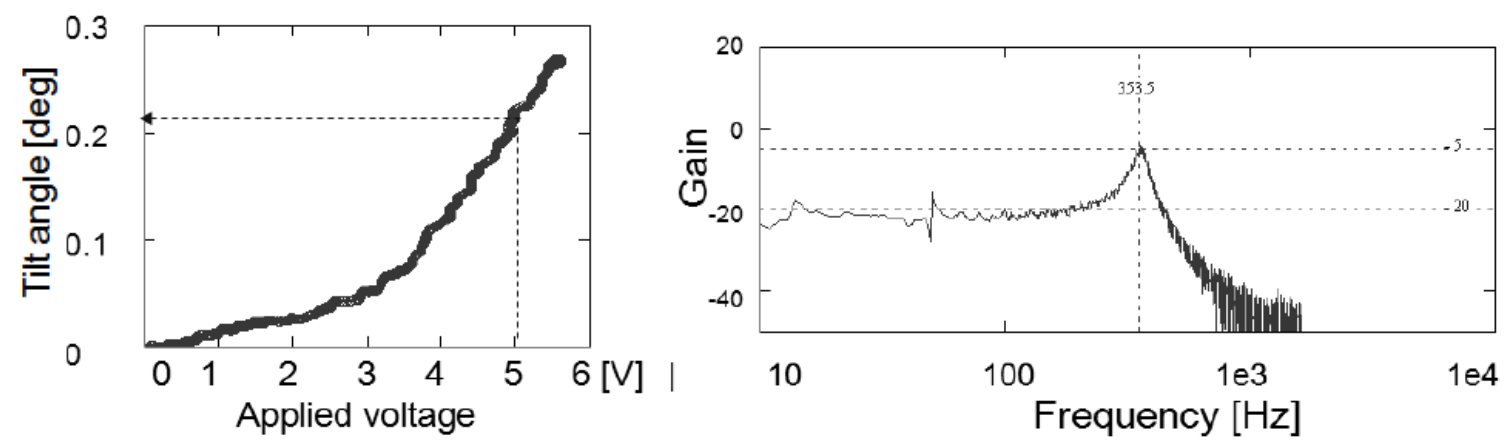

Figure 5. 25 MEMS mirror characteristics: DC tilt angle vs. voltage (left), frequency response (right)

The fabricated MEMS scanning endoscope consists of a lens, a WDM bandpass filter, a photovoltaic detector, and a MEMS scanning mirror packaged in a cylindrical tube as shown in Fig.5.27. The Photovoltaic detector and MEMS mirror are mounted on a glass submount and connected electrically. WDM filter is aligned so that input light is divided into transmission toward MEMS mirror and reflection toward the photovoltaic detector. A focus lens with $3.26 \mathrm{~mm}$ focal length is placed before MEMS mirror so that the probe light focuses right on the circumference of the tube. The assembled optics block on the submount is then inserted into the Pirex glass tube and sealed with the lens housing. This package is completely self-contained so it is easy to realize a hermetically sealed package for reliability and safety. 


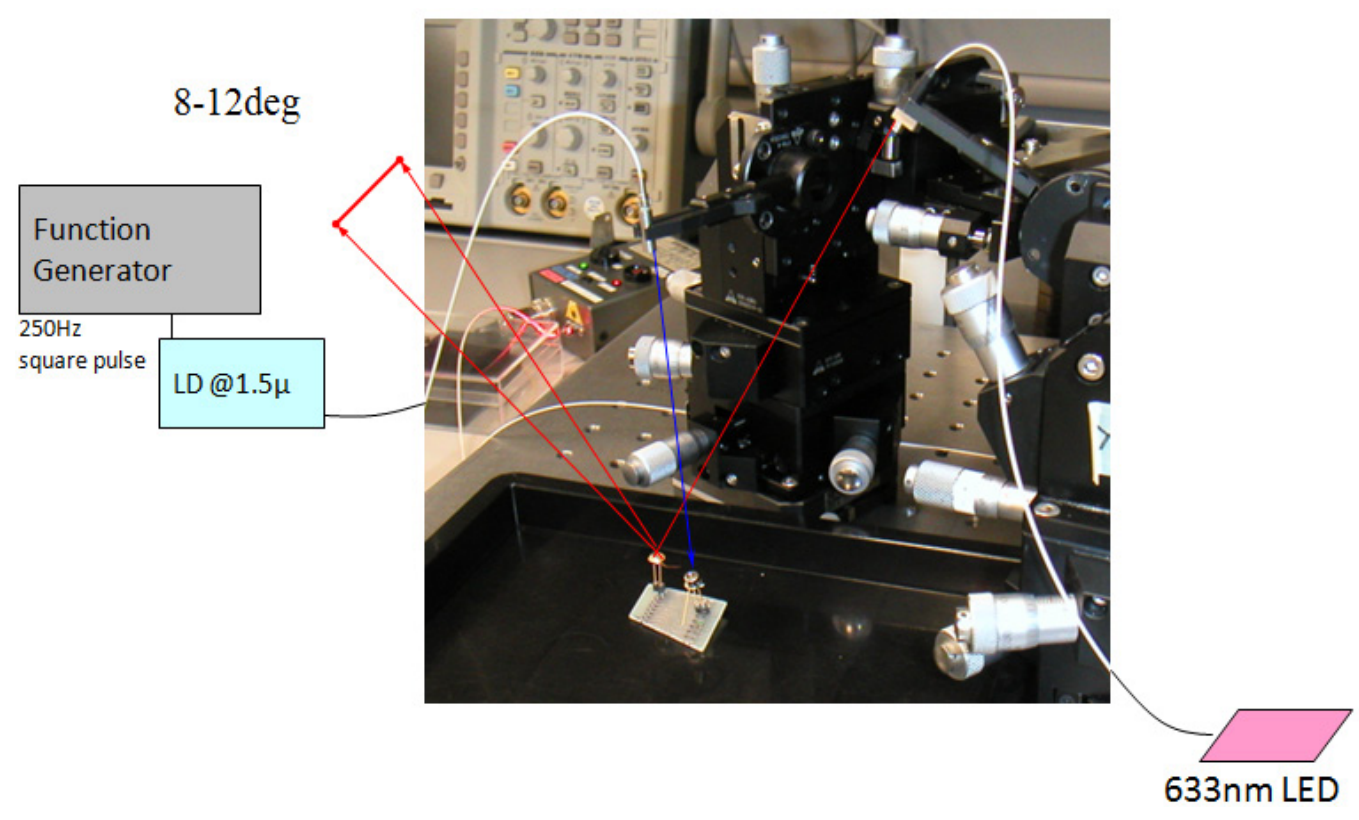

Figure 5.26 Experimental setup for validating the optical actuation
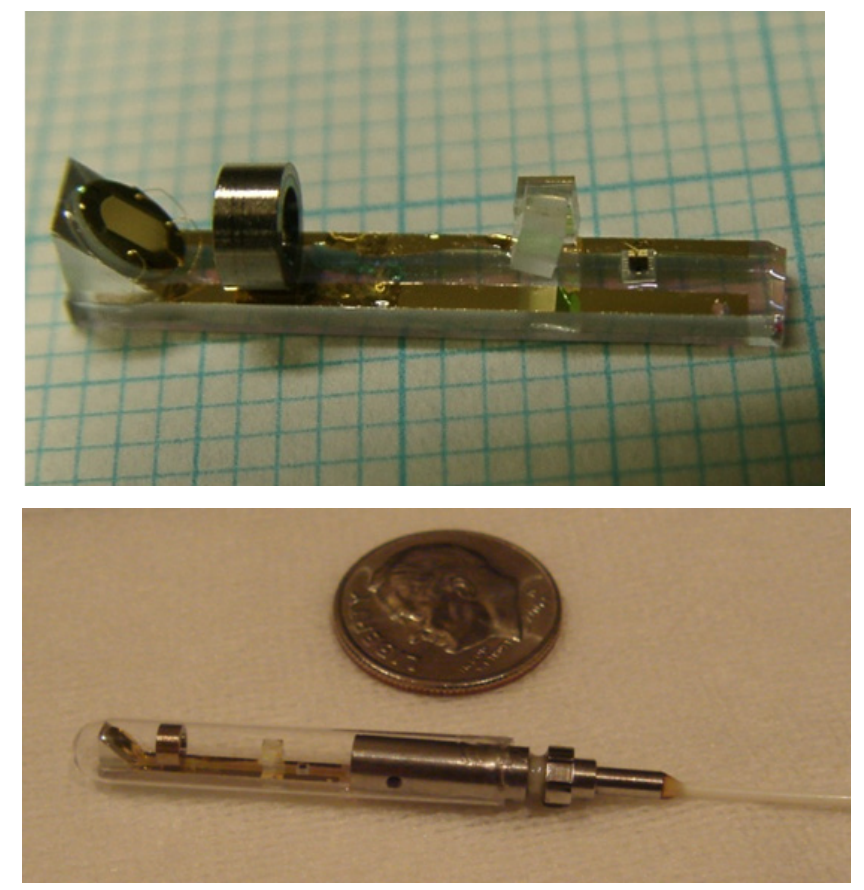

Figure 5.27 Photograph of fabricated MEMS fiber probe:

Core elements mounted on the strip of glass (Top), The device encapsulated in the Pyrex capillary tube with fiber pigtail 


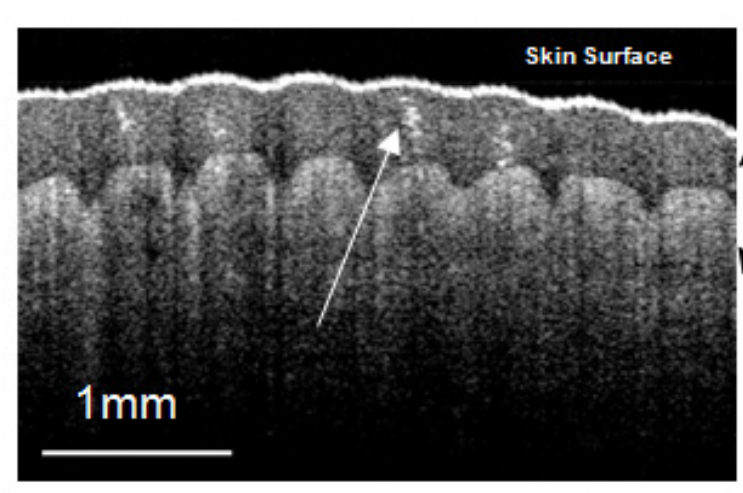

(a)

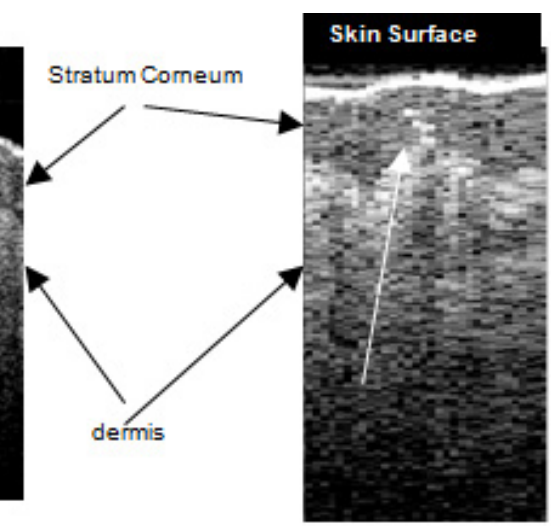

(b)

Figure 5.28 SS-OCT image taken with handheld probe with Galvano mirror (a) and an image measured with the fabricated MEMS mirror (b).

\section{Discussion}

We have demonstrated an optically modulated MEMS mirror based scanning endoscope. A scanning angle of $+/-4 \mathrm{deg}$ at a rate of $300 \mathrm{~Hz}$ was achieved. The scanning angle we achieved is limited by two factors of the present design. First, air-damping introduced by the MEMS mirror acts to reduce the amplitude of the scan. Making the mirror smaller can reduce the air-damping effect. The other reason is the limited electrostatic force. The force applied by the comb drive can be improved by increasing the photovoltage generated by the photovoltaic detector and by increasing the size of the comb drive. There is a room for improvement to realize 45 to $90 \mathrm{deg}$ angle scanning by optimization of design.

The same concept applies to the scanning in different direction other than radial. Transverse scanning is possible simply by switching the axis of the MEMS mirror. In this case, factor of $\mathrm{X} 2$ accounts for the relation of optical to mechanical scanning angle.

Forward scanning is also possible by implementing a number of reflections inside the module. 
Further improvement

A device with improved performance was further investigated. In this prototyping, a MEMS scanning mirror (Fraunhofer Institute (Hiperscan Inc.), MSS1D) [101] with a large scanning angle was used as shown in Fig. 5.29. Typical specification is listed in Table 5.2.

Table 5.2 MEMS mirror (MSM1D) specification

\begin{tabular}{|l|l|l|}
\hline Parameters & Specification & Notes \\
\hline Device & $1 \mathrm{D} \mathrm{scanner}$ & \\
\hline Mirror diameter & $1.5 \mathrm{~mm}$ & \\
\hline Resonance frequency & $250 \mathrm{~Hz}$ & Drive frequency 500Hz \\
\hline Scan range & $60 \mathrm{deg}$ (optical)@20V & At normal pressure \\
\hline Reflection coefficient & $88 \%$ & \\
\hline Chip size & $2.9 \mathrm{~mm} \times 4.3 \mathrm{~mm}$ & \\
\hline Shock resistivity & $>2000 \mathrm{G}$ & When not operating \\
\hline
\end{tabular}
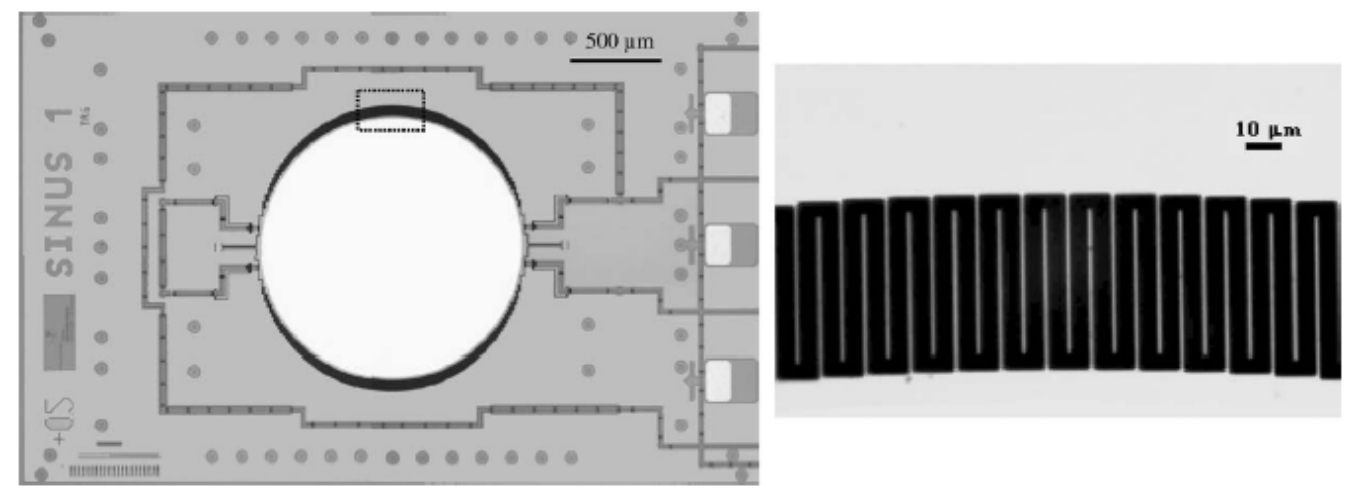

Figure 5.29 Photograph of MEMS mirror

In order to make a full advantage of scan range of this MEMS mirror, we needed to improve the voltage generated from photovoltaic cell. In this case, we used a photovoltaic cell with new design that has been integrated in parallel with multiple cells around the effective illumination area as shown in Fig. 5.30(a). The structure of each cell is similar to that depicted in Fig.5.22. Four cells are all connected via the electrodes to simply multiply the voltage generation by a factor of four. Figure 5.30 (b) shows improved performance of photovoltaic cell with about $14 \mathrm{~V}$ output voltage when $10 \mathrm{~mW}$ power was focused onto the center of photovoltaic cell with about $100 \mu \mathrm{m}$ beam diameter. Estimated scan angle at $14 \mathrm{~V}$ is 45 degree in optical scan with new MEMS mirror. 
Instead of Pirex glass capillary plastic tube (PTF) is used as tube material for the probe. PTF of $6 \mathrm{~mm}$ outer diameter, $5 \mathrm{~mm}$ inner diameter is used, which is common material for conventional catheter. A diagram of improved design is shown Fig. 5.31. The device is sealed with medical grade UV epoxy (LOCTITE3021) that can pass ETO sterilization process. The rest of basic operation principle is same as initial sample except for the actuation mode of MEMS device. Since this MEMS mirror doesn't have offset angle like the previously fabricated one as seen in Fig.5.29, start of actuation is little bit tricky that you need to sweep the modulation frequency from the value higher than resonant frequency toward resonant frequency to 'trigger' the resonance of the mechanical oscillation.
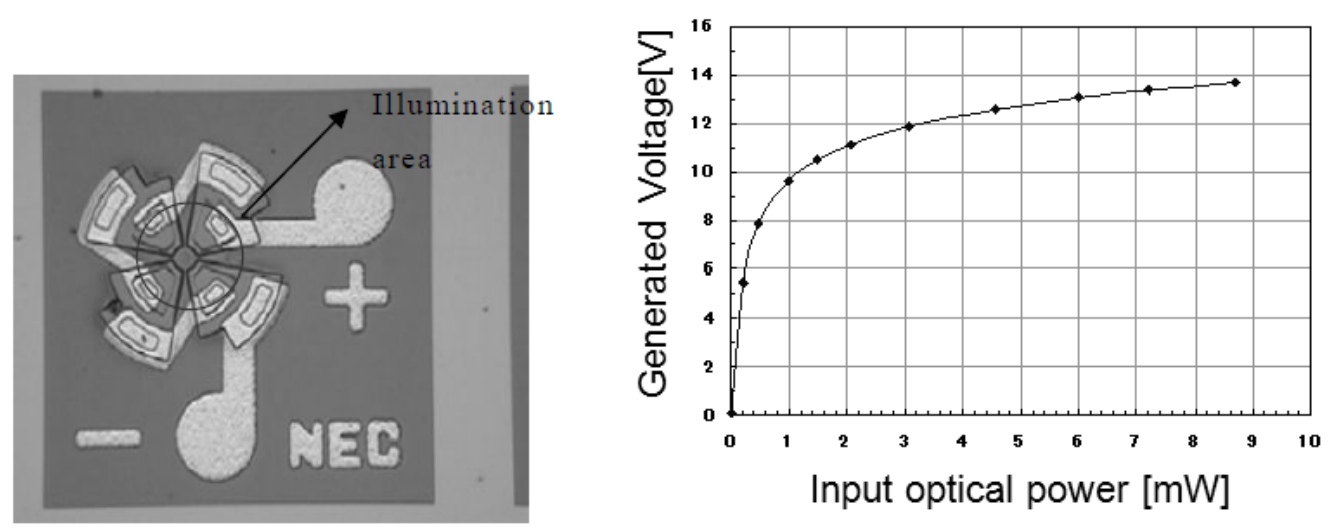

Figure 5.30 Photograph of Photovoltaic cell and its voltage vs optical power (in courtesy of NEC devices)

\section{Results}

To demonstrate the optical modulation, a test similar to the previous mode was carried out, but this time with modulation signal at $1550 \mathrm{~nm}$ actually propagated via single mode fiber and split and reflected onto the photovoltaic cell by WDM bandpass filter (Fig. 5.32). Visible light in red is projected onto the mirror to scale the scan angle of reflected beam. Measured angle was about $40 \mathrm{deg}$ in optical scan with modulation signal with $15 \mathrm{~mW}$ peak optical power at $500 \mathrm{~Hz}$ square waveform. $40 \mathrm{deg}$ angle corresponds to about $2 \mathrm{~mm}$ peripheral length in arc provided that focus is just outside the outer diameter of the tube, which gives a relatively wide field of view. 


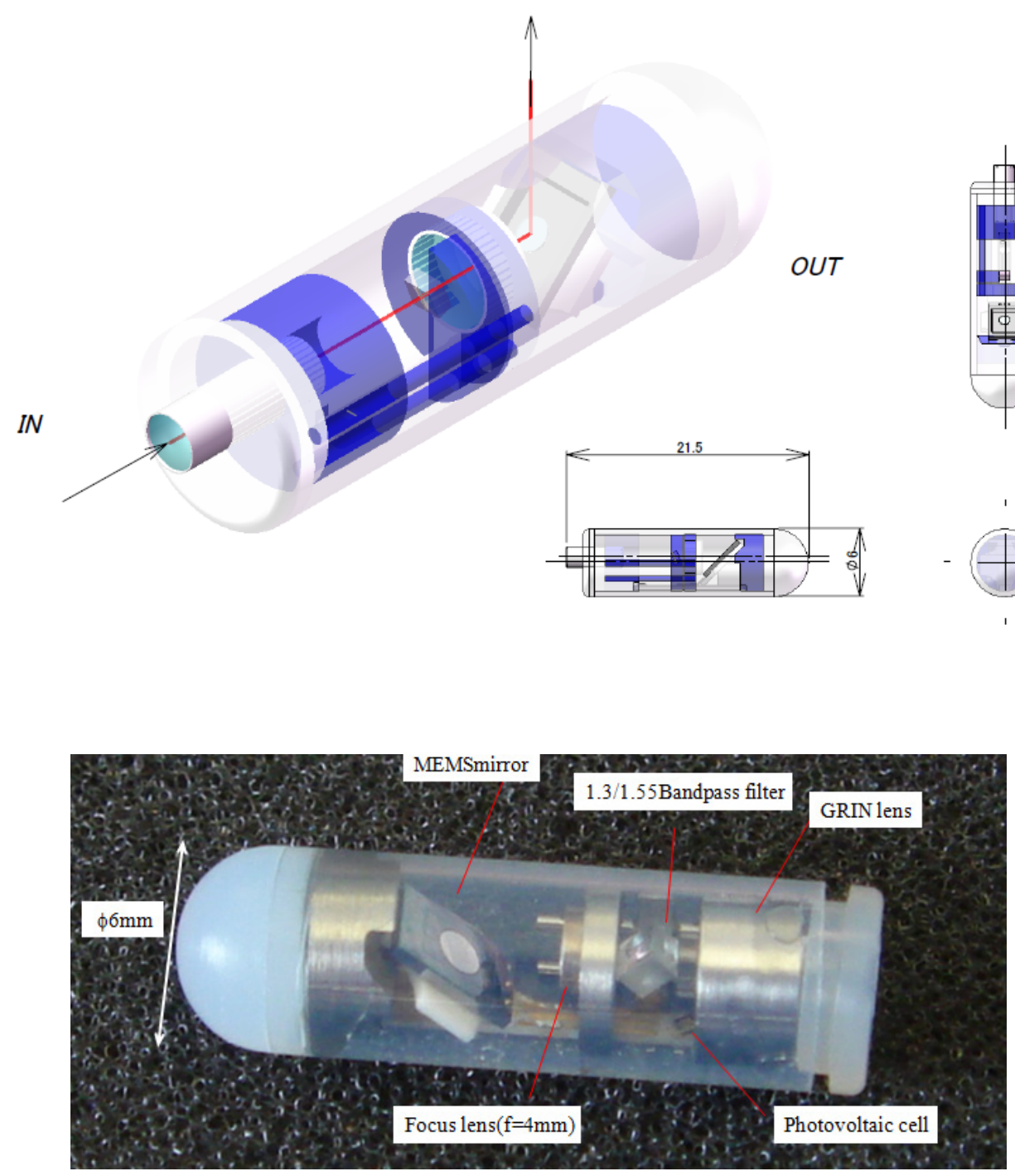

Figure5.31. Diagram of improved MEMS probe design(Top) and photograph the fabricated sample (bottom) 


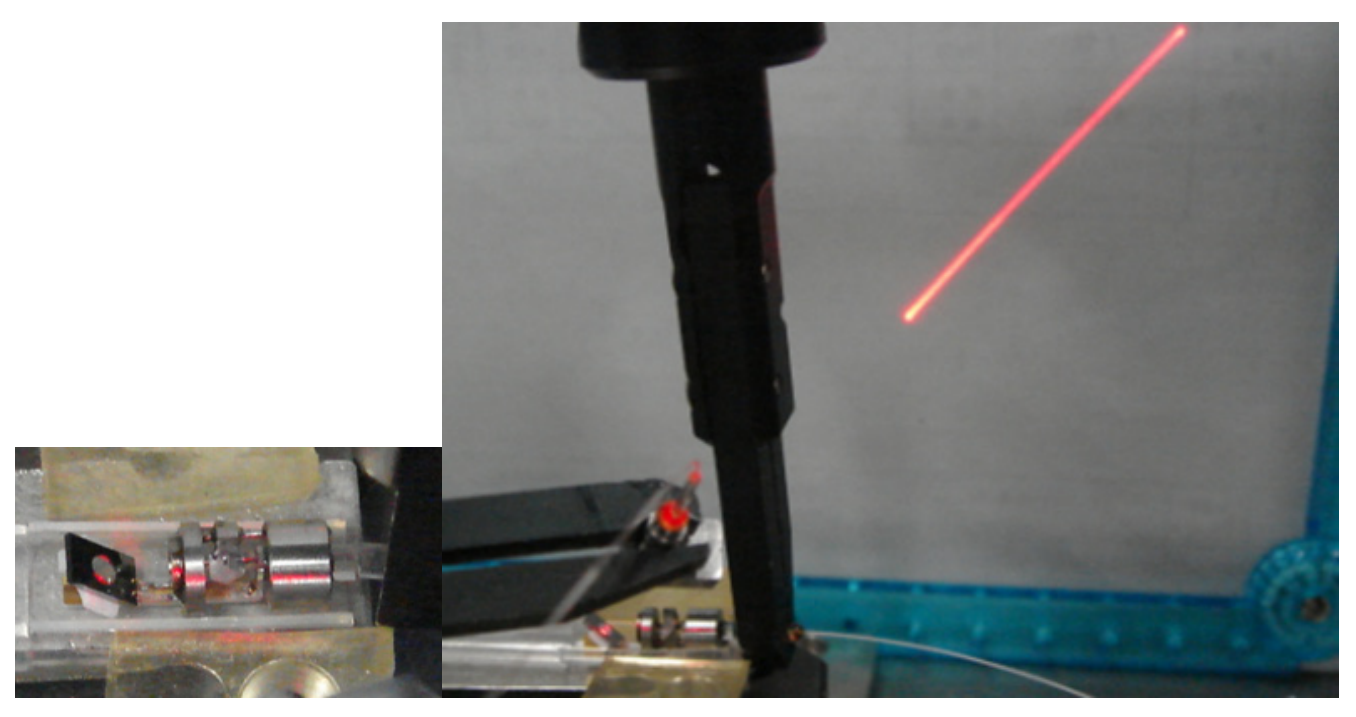

Figure 5.32 Experimental setup for validation of optical modulation

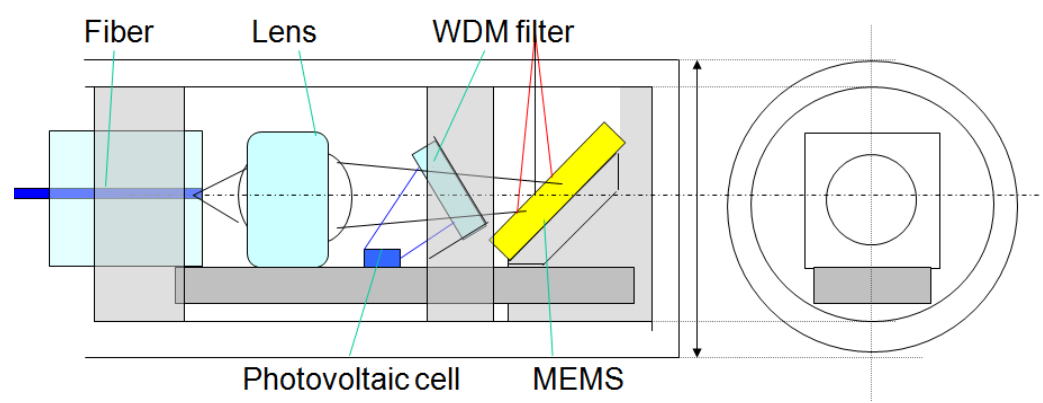

Figure 5.33 Future design consideration of compact MEMS probe 


\subsection{Summary}

SS-OCT system description was disclosed and a number of clinical data were discussed such as in oral tissues and ophthalmic samples. Even though SS-OCT has advantages over conventional TD-OCT in principle, there should be more rigorous and much larger number of clinical evidence required to support or to prove its advantages [126,127] in the future. One disadvantages of SS-OCT or general OCT system is difficulty in imaging highly or multiple scattering mediums such as tissue or cancer as well as caries of teeth [128-132]. This should be countermeasured in the future by applying further longer wavelength range. In the last section, a new concept of optically modulated MEMS probe was explained. A novel endoscope incorporating a low voltage driven MEMS scanner and photovoltaic detector wss proposed. Our approach, that utilizes an optically modulated MEMS scanning mirror, eliminates the need for external electrical voltage source. This concept provides an option of the endoscopes scanning operation to be controlled remotely without introducing high voltage signals and wires that can be detrimental to in-vivo diagnosis. We have demonstrated an optical scan angle of 45 degrees by utilizing $15 \mathrm{~mW}$ of $1550 \mathrm{~nm} \mathrm{light}$ modulated at $500 \mathrm{~Hz}$ to actuate a MEMS mirror for $250 \mathrm{~Hz}$ scan. Further improvements and optimization of the design for increasing scan angle, and the development of a compact module are necessary for practice in clinical applications. In the future design, a single lens will be used and the band separation is done in focusing path of the light as shown in Fig.5.33. In this way, whole module can be more compact and sensitivity of photovoltaic detector will be further improved. This approach should benefit when OCT is used as combined or complementary modality with the other imaging systems. 


\title{
Chapter 6 Feasibility study of single mode continuous fast sweep
}

\author{
Introduction \\ In the previous chapters, basic swept sources all have several millimeter or centimeter order \\ coherence length during high-speed sweep. As explained in Chapter 1 Introduction, \\ integrated devices such as DFB and SSG-DBR or "single mode oscillation lasers" do not \\ meet full requirements of swept source OCT. However, integrated devices are not only \\ advantageous when the market volume is large and cost pressure is high for its mass \\ production capability for OCT applications but also that its single mode oscillation provides \\ coherence length in kilometer that may open up new applications such as dynamic gas \\ analysis, fiber sensing interrogators. So ideally, compact, low-cost, fast sweep single mode \\ continuous source is ultimate goal of this research. In this chapter, potential outlook of three \\ promising technology candidates is introduced and briefly overviewed.
}




\subsection{MEMS VCSEL}

Vertical Cavity Surface Emitting Laser diode (VCSEL) is intrinsically single mode oscillation because of such short cavity length between two DBR mirrors built in the vertical direction inside the wafer. Tunable VCSEL [103] has been studied over decades at various wavelength ranges that overlap with the usable range for OCT.

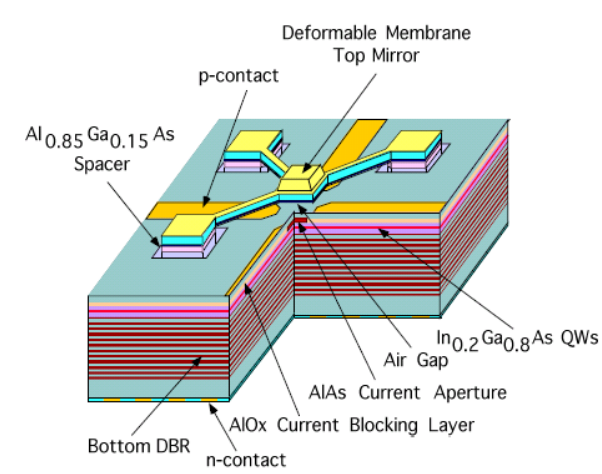

Figure 6.1 Conventional Tunable VCSEL [13]

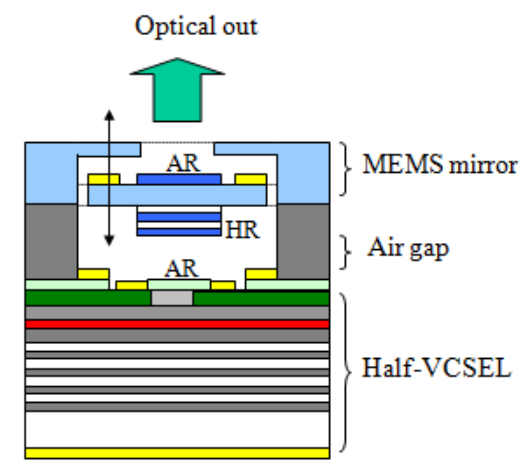

Figure 6.2 Proposed MEMS hybrid tunable VCSEL

Although the output power of current injection type VCSEL with single lateral mode has relatively lower power, continuous tenability with just one control node (i.e. bias voltage to the deformable membrane) enables easy control of repetitive sweep at high-speed. However, monolithic structure where top DBR mirror is made of 3-5 semiconductor compounds has difficulty in manufacturability [104]. Recently there have been several reports on hybrid structure combined with bulk silicon MEMS membrane on top of half VCSEL [105]. However, control of gap in between top DBR of silicon MEMS and bottom half VCSEL has been difficult in terms of complex actuation mechanism of membrane. In order to make a simple and accurate control of the gap for defining FSR and tuning range, we proposed a vertically movable MEMS mirror that individually has electrostatic electrodes on the MEMS side only as shown in Fig.6.2. In this way, optical cavity and electrostatic gap are separated to have individual and not cross-interference in control. The MEMS vertical mirror is designed in similar process that was used for MEMS mirror for the fiber probe. SOI wafer was simply dry-etched from both sides and sacrificial etching was performed.

Figure 6.3 shows preliminary results of vertical translation against applied voltage measured by laser Doppler interferometer. According to the simple design theory of Fabry-Perot etalon filter, FSR and displacement of MEMS mirror are related to initial air-gap length as in Fig. 6.4. 


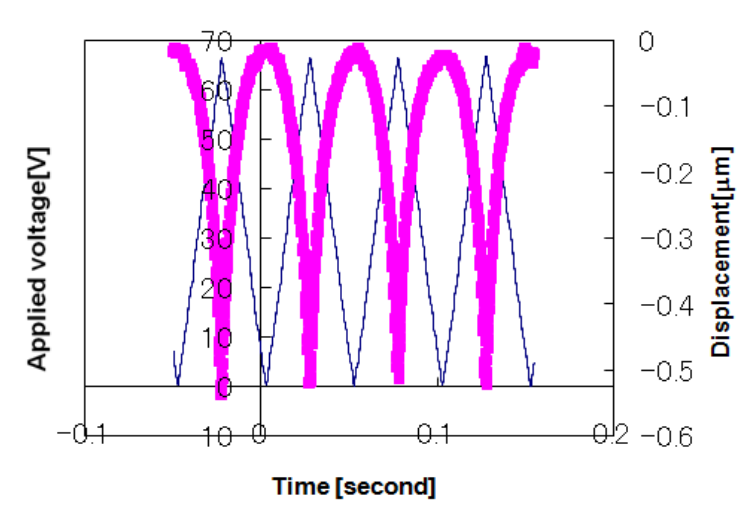

Figure 6.3 Relation of applied voltage and displacement over time

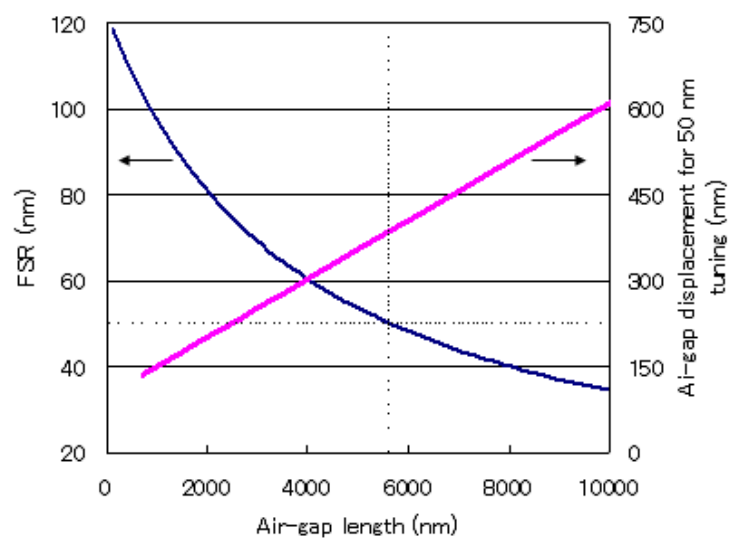

Figure 6.4 Relation of FSR and Air-gap vs. air-gap length

Further study and results will be reported elsewhere in the near future. 


\subsection{Integrated ECL}

Integration of ECL with the same configuration that was discussed in the previous chapters into single ship device is the most ideal approach if the proportional configuration can be realized in very small footprint. Similar idea was proposed by different group [106,107] where EO beam deflector is built into the LD substrate by using refractive index change induced by injection current. But an effective electro-optic coefficient is not as large as nonlinear optic crystals like LN or PLZT having over several tens of $\mathrm{pm} / \mathrm{V}$ in coefficient. On the other hand, EO deflector made into slab waveguide by EO polymer materials [108] or by solid-phase epitaxy [109] have practically large EO coefficients over 50 to $100 \mathrm{pm} / \mathrm{V}$.

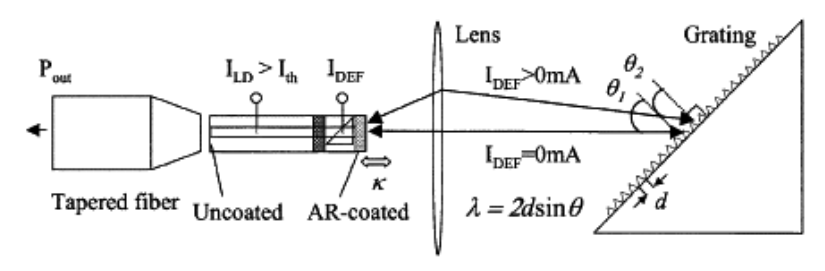

Figure 6.5 Proposal of EO deflection for tunabiligy [106]

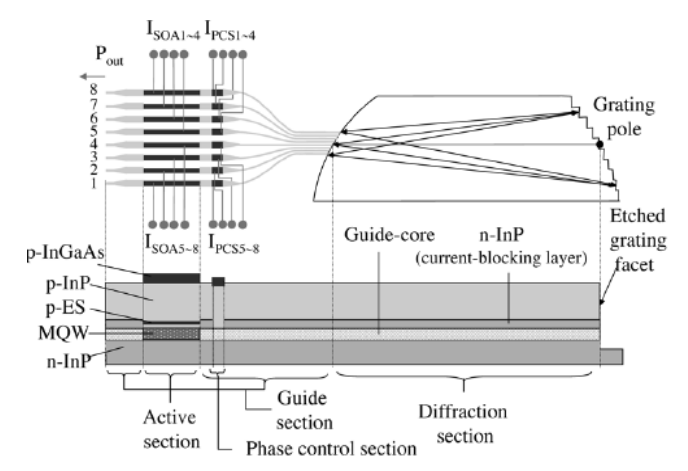

Figure 6.6 Monolithic EO tunable ECL [107]

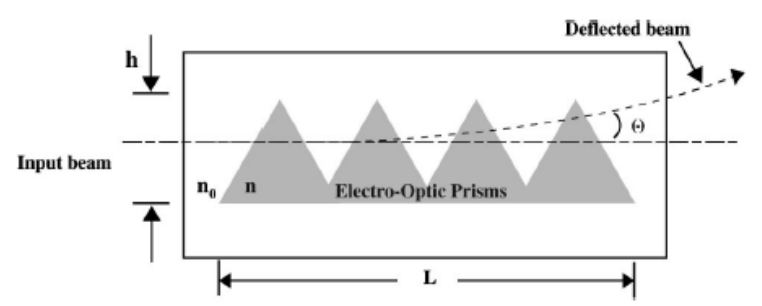

Figure 6.7 Polymer EO deflector with cascaded electrode section [108]

\section{Single mode continuous tuning}

EO deflectors based on nonlinear materials can realize nanosecond order response time and a few degree of beam deflection. However, arrangement for pivot tuning cannot be realized in terms of geometry so that single mode continuous tuning is not that simple forward.

We address the novel technique to amend the design limit by taking into account that refractive index change in EO deflector does not only change the angle of deflection but also change the optical path length. We used this modulation to compensate the phase lag between wavelength change by grating filter and longitudinal mode variation by deflection 
angle, which is analogous to the QPCT technique. While, this is to find completely synchronous condition taking into account with refractive index change.

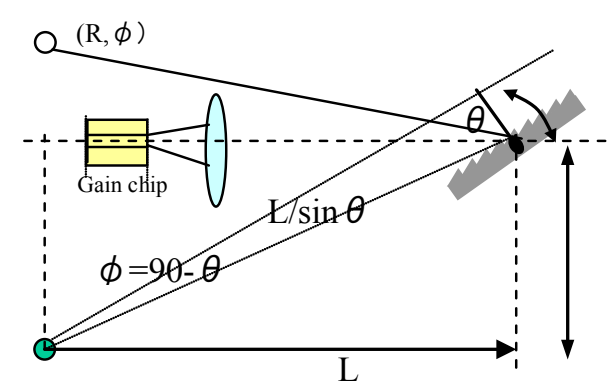

Figure 6.8 (a) Pivot tuning

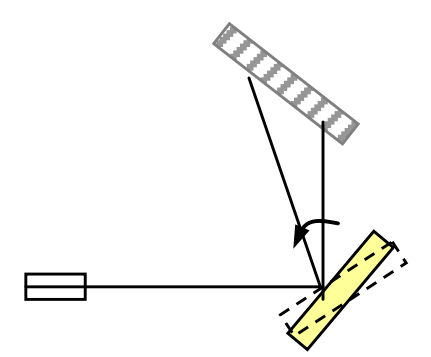

(b) Non-pivot tuning as in QPCT
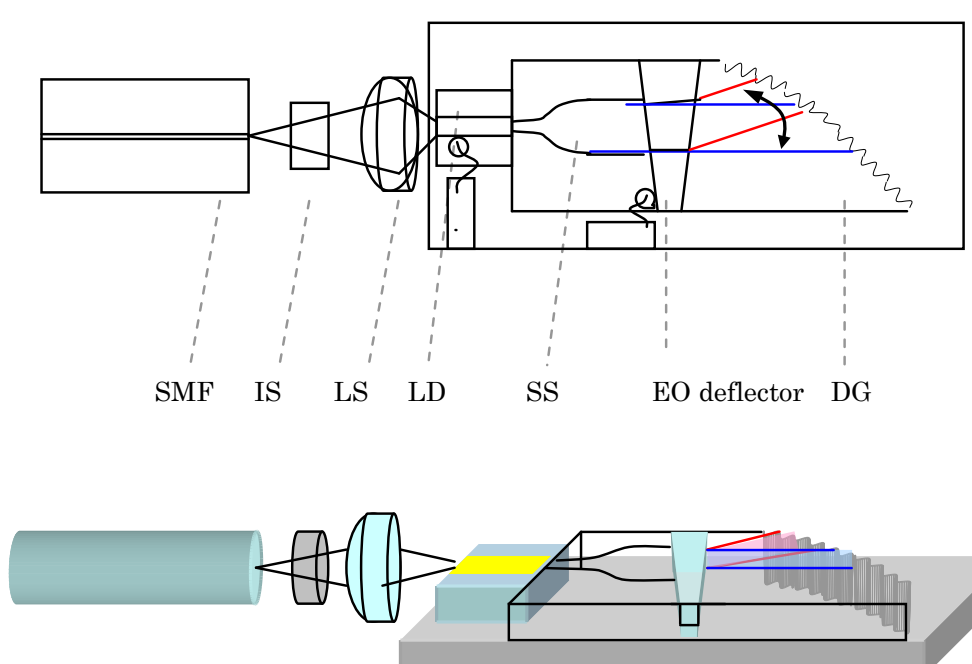

Figure 6.9 Proposed Hybrid EO deflector ECL type swept source

IS: Isolator, LS:Lens, LD;Gain chip, SS: Spotsize converter, DG Diffraction grating

Condition can be analytically derived by solving the simple Snell's law on the boundaries of EO deflector and its wavelength of longitudinal mode $(\lambda \mathrm{m})$ along the diffraction angle can be expressed in Eqs. (6.1)

$$
\begin{gathered}
\lambda_{m}=\frac{\lambda_{o}}{L_{o}}\left[L_{1}+b \frac{1}{\cos (\alpha-\theta)}+\frac{L_{2}+b \tan \theta_{0} \tan (\alpha-\theta)}{\cos \phi-\sin \phi \tan \theta_{0}}\right] \\
\theta=\arctan \left\{\frac{\sin ^{2} \alpha}{\sin \phi+\sin \alpha \cos \phi}\right\} \\
L_{e f f}=L_{1}+b \frac{1}{\cos (\alpha-\theta)}+\frac{L_{2}+b \tan \theta_{0} \tan (\alpha-\theta)}{\cos \phi-\sin \phi \tan \theta_{0}}
\end{gathered}
$$


where each parameter is defined in Fig. 6.10.

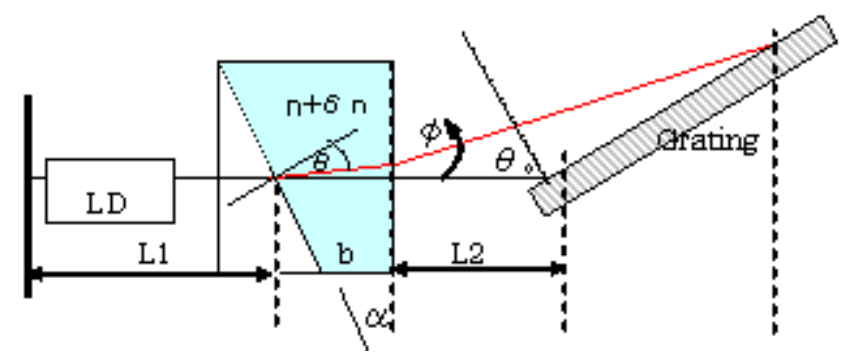

Figure 6.10 Definitions of geometrical parameters

It is difficult to solve the equation analytically, however we found that with an appropriate conditions yield single mode hop free, or continuous tuning over $20 \mathrm{~nm}$ at $1300 \mathrm{~nm}$ range.

Here we use the criteria of $|\lambda \mathrm{g}-\lambda \mathrm{m}|<\mathrm{c} / 4 \mathrm{~L}$ as a mode hop free condition.

Figure 6.11 shows the degree of phase synchronization between longitudinal mode and filter wavelength in the case of non-pivot tuning as in Fig. 6.8(b). Here, at least mode hops should occur about 10 times over a single sweep. While, if we optimize the condition, mode hop will never occur as shown in Fig. 6.12 , where $\Lambda=1200 \mathrm{~g} / \mathrm{mm}, \alpha=30 \mathrm{deg}, \lambda_{\mathrm{o}}=1260 \mathrm{~nm}$, $\mathrm{L}_{1}=8 \mathrm{~mm}, \mathrm{~L}_{2}=10 \mathrm{~mm}, \mathrm{~b}=3.86 \mathrm{~mm}$.

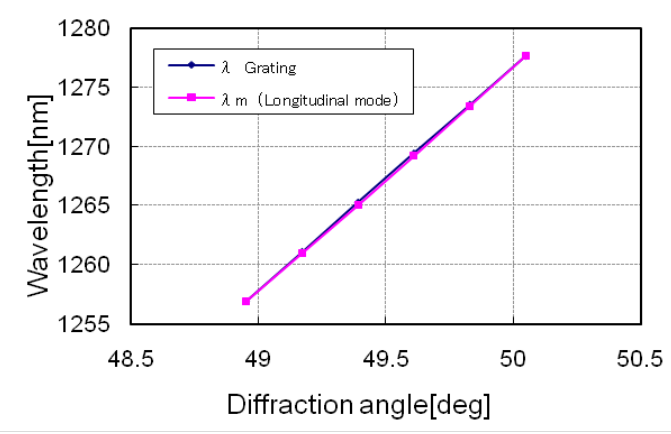

Figure 6.11 (a)Phase change vs. diffraction angle

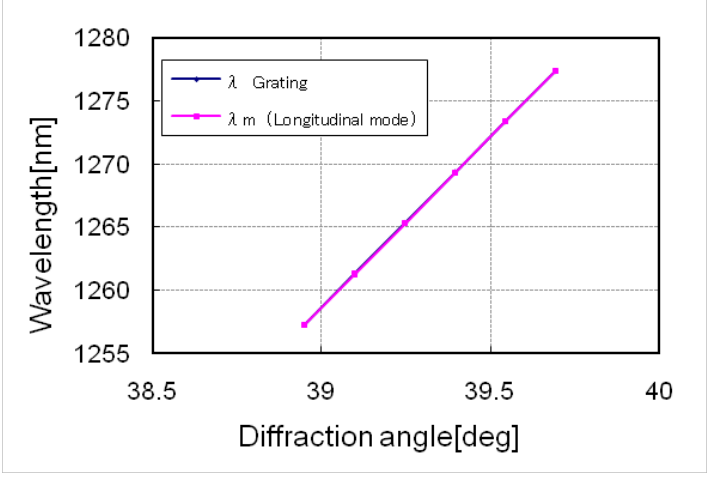

Figure 6.12 (a)Phase change vs. diffraction angle

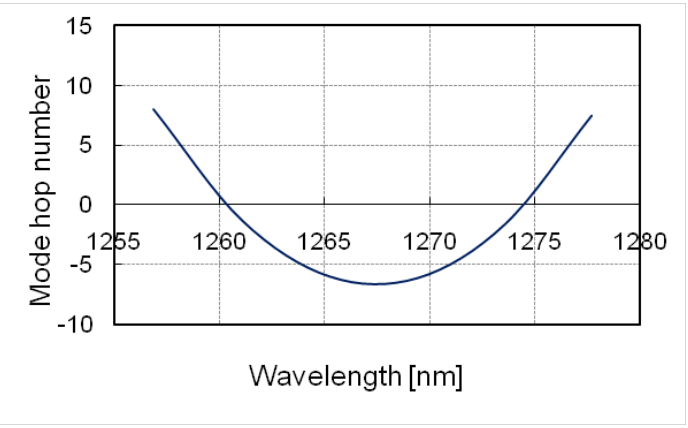

(b). Mode hop number

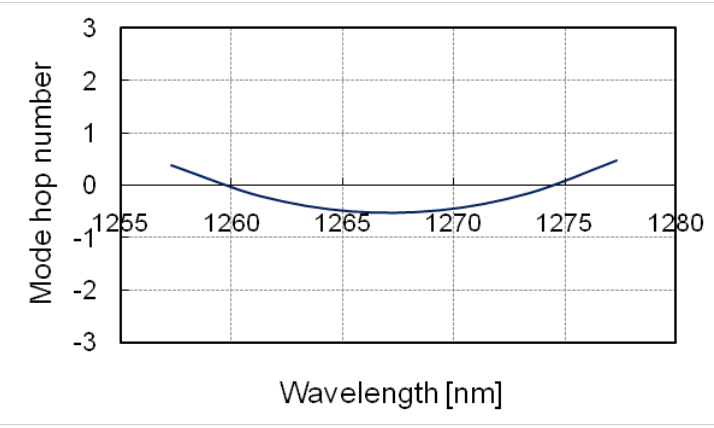

(b) Mode hop number 


\subsection{Fiber ECL with saturable absorber}

Last candidate is a fiber extended cavity laser with saturable absorber filter which acts as a narrow bandpass filter that automatically tracks the lasing wavelength, thus ensuring the single longitudinal mode operation [110-113] (Fig.6.13). Unpumped erbium doped fiber (EDF) can be used for this purpose at $1550 \mathrm{~nm}$ band with additionally wavelength tunable filter. Standing wave caused by counter propagating light will self-induce the fiber Bragg grating along the EDF and the center wavelength is tracked with lasing wavelength. If cavity modes are finely packed within the wavelength range and FBG filter is narrower than cavity mode spacing, each adjacent cavity mode is consecutively oscillated, thus quasi-continuous sweep is possible with no mode partition or bistability. Gain medium used in previous work is EDFA so that sweep speed is limited by response time of EDFA resulting in submilisecond or swept rate less than $\mathrm{kHz}$ order. If $\mathrm{SOA}$ is implemented as a gain medium, much faster swept rate over several $\mathrm{kHz}$ can be expected by using same configuration we used for the study in previous chapters as shown in Fig.6.14.
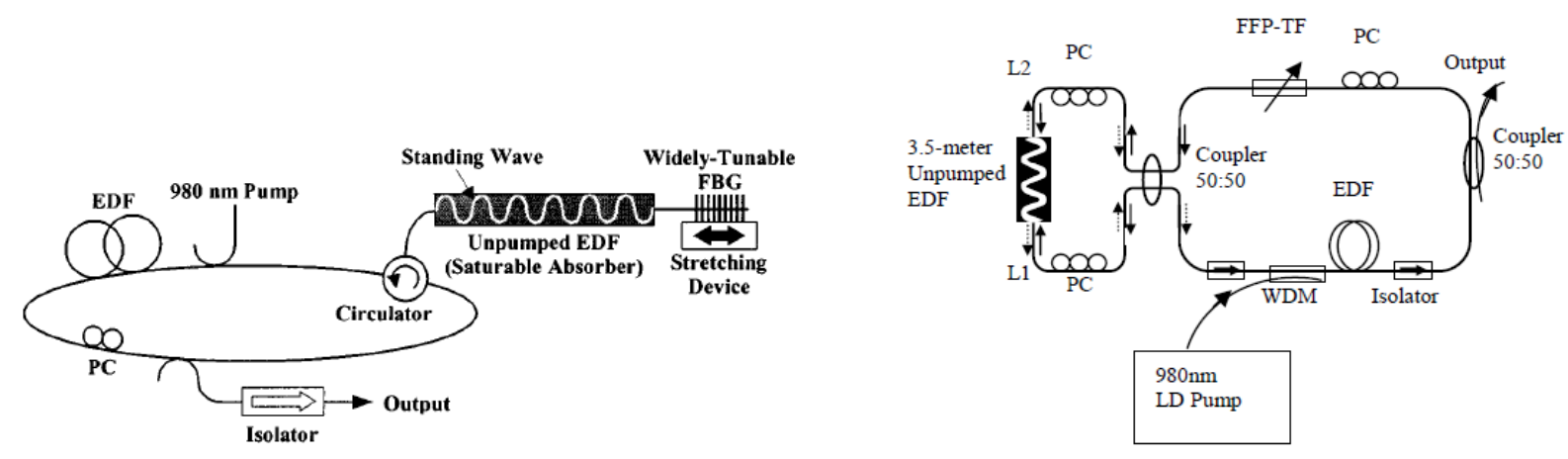

Figure 6.13 Single longitudinal mode tunable fiber laser with a self induced saturable absorber filter ([110],[111])

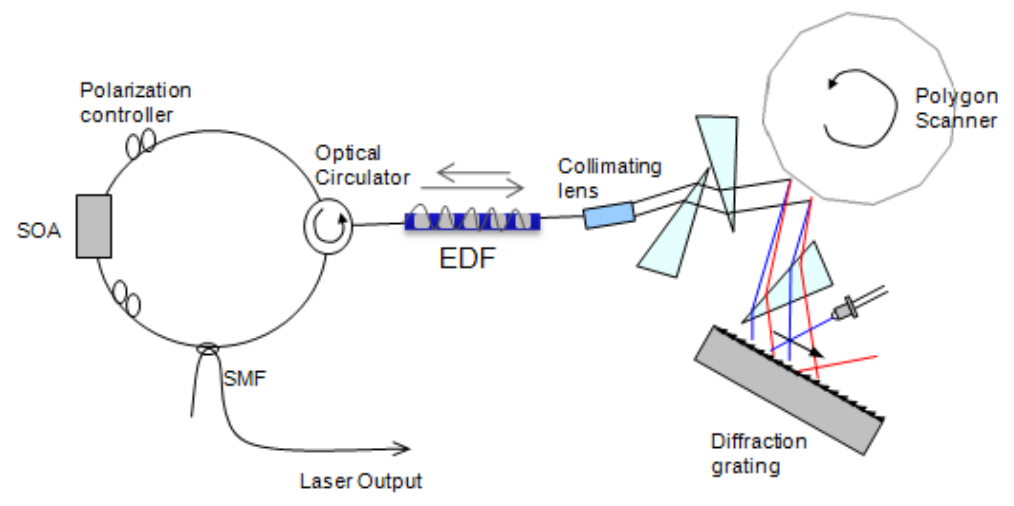

Figure 6.14 Proposed configuration with saturable absorber filter

Further investigation and experiment is left for future work and will be reported in the future. 


\subsection{Summary}

Single mode continuous tenability is of great importance in many applications. In this chapter potential technologies are discussed for future study. While hybrid tunable MEMS and hybrid ECL are the ultimate or ideal device mode for mass productivity, fiber ECL is the shorted cut to meet the immediate application needs where larger coherence length or narrower linewidth is important to overcome the measurement resolution. 


\section{Chapter 7 Conclusion}

In this thesis, correlation of characteristics of swept source is investigated in different point of views. Several new techniques are proposed and validated via constructing SS-OCT images using proposed swept source.

Major outcome throughout this study are followings;

1. Littrow based diffraction grating tunable filter combined with a fiber extended cavity laser source is implemented to realize high-speed wideband swept source. Stable operation of over $120 \mathrm{~nm}$ swept range and $15 \mathrm{~mW}$ output power has been confirmed with fabrication the prototype laser. Compensation optics by using a prism expander in an optimum arrangement enables 0.05 to $0.2 \% \mathrm{k}$-linear sweep so that it can eliminate the wavelength rescaling process that was a heavy burden to the system process time. As a result, it opens up a possibility of real time imaging of tomography.

2. Coherence length improvement was realized by novel concept called "Quasi-Phase Continuous Tuning" technique. Theoretical analysis was established to estimate the dynamic spectral linewidth of QPCT operation assuming Quasi-stationary oscillation. Here, multimode rate equations are introduced and extended to carry out the linewidth analysis. Simple rearrangement of Littrow configuration yields coherence length improvement or spectral narrowing effect by a factor of two in comparison with the case not using QPCT. This technique was further carried out for large coherence length swept source for scaling applications such as intraocular distance measurement. Using $1 \mu \mathrm{m}$ swept source combined with QPCT successfully performed OCT imaging in $20 \mathrm{~mm}$ range.

3. Various approaches to overcome the design trade offs between swept range and coherence length at high speed sweep were proposed. We proved that spectral shaping and injection current modulation are effective to take full advantage of wide gain band of SOA. Both techniques can expand the bandwidth without worrying the issue of COD. Double pass and reflection configuration were also proposed and found that both configuration is effective to countermeasure the design limit of tunable filter using 
diffraction grating and polygon scanner. Maximum of $170 \mathrm{~nm}$ tuning range was demonstrated with the above method. Double reflection configuration enabled $100 \mathrm{~nm}$ wide range sweep at $50 \mathrm{kHz}$ swept rate while maintaining $5 \mathrm{~mm}$ coherence length during the sweep. $50 \mathrm{kHz}$ fast sweep proved the fast acquisition of three-dimensional tomograph.

4. SS-OCT imaging was carried out in clinical studies in oral tissues and ophthalmic area by developing a system with interferometer and probe that proves the benefits of present inventions with the swept source. Especially, $1.3 \mu \mathrm{m}$ range source is found out tot be suitable for anterior chamber measurement.

5. Novel optically modulated MEMS probe is proposed and fabricated. It has a potential of non-hazardous diagnosis in endoscopic applications.

6. Further possibility of single longitudinal mode continuous tuning is discussed. Three major areas of potential technologies are addressed with initial feasibility study results.

This outcome of this study should accelerate the commercialization of SS-OCT in diagnostic equipment in the near future. And it should also broaden the applications such as industrial inspection system, sensing system as well as basic biological study. 
APPENDIX: SS-OCT IMAGE GALLERY 
In the Appendix, various image samples are shown with brief explanation. These are works done by OCT research groups all over the world who implemented the developed swept sources based on present invention described in this thesis. Examples of biological samples and industrial samples are measured with our SS-OCT system described in Chapter 5.

(1)Retinal imaging with $1 \mu \mathrm{m}$ swept source

This work was done at Tsukuba Univ. [141]

The center wavelength is $1060 \mathrm{~nm}$, the scanning range is $72 \mathrm{~nm}$, maximum power of $4 \mathrm{~mW}$ at peak. Swept rate of the source is $28 \mathrm{kHz}$.

\section{(a) $1.05 \mu \mathrm{m}$}

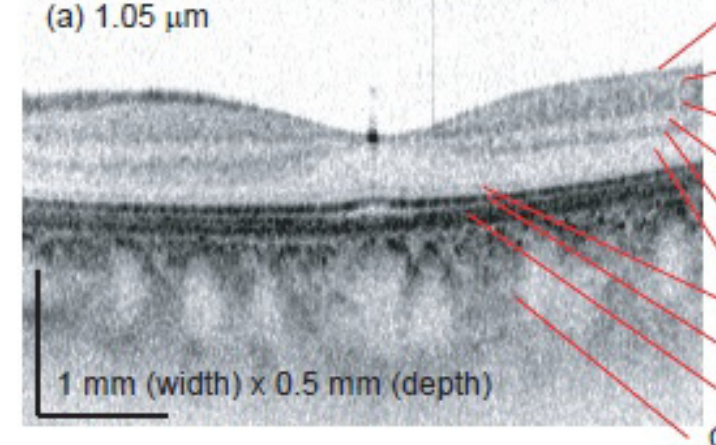

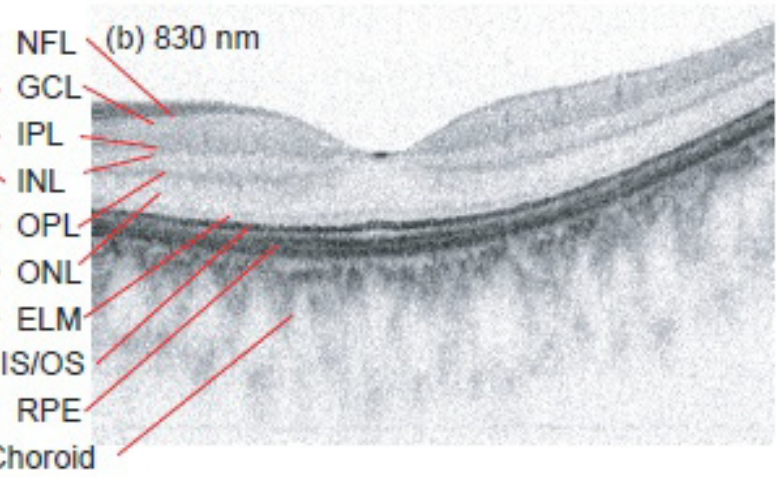

Figure A.1 OCT B-scan of in vivo human macula captured using (left) 1- $\mu \mathrm{m} \mathrm{OCT}$ and (right) 830-nm SD-OCT. (Image taken from ref [141])

It shows a comparison of the B-scan images of the in vivo human macula captured using $1 \mu \mathrm{m}$ SS-OCT and 830-nm SD-OCT [114,115]. In this figure, each individual layer including the nerve fiber layer (NFL), ganglion cell layer (GCL), inner plexiform layer (IPL), inner nuclear layer (INL), outer plexiform layer (OPL), outer nuclear layer (ONL), the junction between the inner and outer segments of the photoreceptor (IS/OS), RPE complex, and choroids are visible.

The better penetration and higher contrast of the choroid obtained from $1 \mu \mathrm{mSSOCT}$ are evident from these images. A strong OCT signal is visible even beneath the choroidal vessels in the $1 \mu \mathrm{m}$ image because of the wavelength dependent scattering property of the choroid. $1 \mu \mathrm{m}$ range also has the smallest dispersion in water so depth-dependent axial linewidth broadening effect due to material dispersion in tissue is minimum to keep the uniform resolution along the depth.

Figure A2 (a) shows 3D volume-rendered OCT images of in vivo human macula in which the OCT intensity signal is displayed in an inverted-gray color map and the choroidal vessels are displayed with an orange-red color map. (b) shows en face slices of the volumetric 
rendering at several different depths, where a semitransparent color map is applied to the OCT intensity volume. (c) A stereoview of the choroidal vessels of the macula.
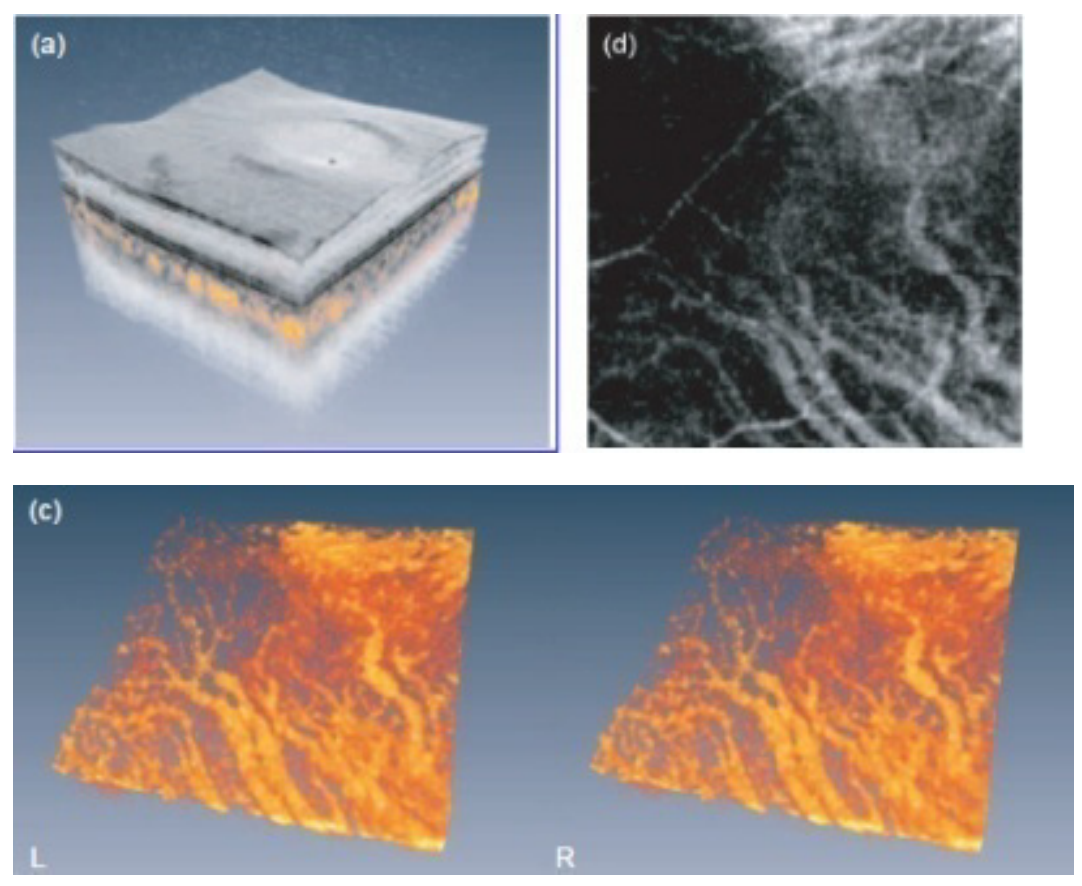

Figure A.2 3D volume-rendered OCT images of in vivo human macula (taken from ref [141])

(2)Anterior segment with PS-OCT using $1.3 \mathrm{~mm}$ swept source $(20 \mathrm{kHz})$

Polarization-sensitive OCT [116-119] that was developed by Tsukuba Univ. group enables the imaging of birefringence of materials. Difference in birefringence of material provides additional layer information to the mere intensity profile.

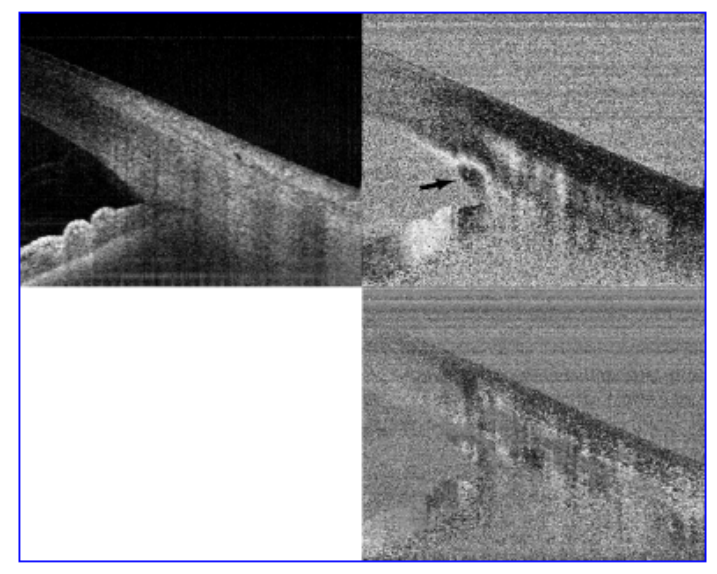

Fig. A3. Intensity (upper left), phase retardation (upper right) and orientation (lower right). Images of the in vivo human anterior eye segment. The size of the images was $5.0 \mathrm{~mm} \times 3.9 \mathrm{~mm}$ in air. (Image taken from ref [144]) 
(3) Endoscopic applications

Endoscope imaging with using a fiber catheter benefits the imaging of the sidewall of the organs [120-123].Figure A4 shows in-vivo OCT image of rabbit trachea that has good agreement with histology image (d) (J. Su, ZP.Chen, et al. of Univ. of California, Irvine)[140,142,147]. Figure A5 shows the image of oral cancers that was measured by the group of National Taiwan University [151,152].

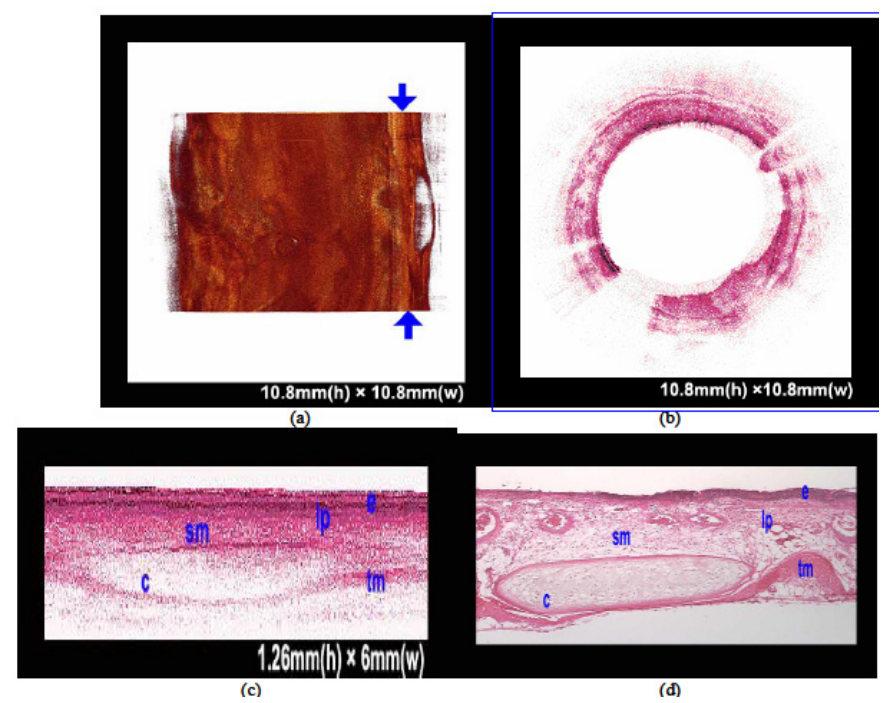

Fig. A4 (a) Three-dimensional rebuilt image of rabbit trachea which was $6 \mathrm{~mm} \mathrm{long}$; the trachea ring is visible in the middle part. (b) One cross-sectional slice pulled out from (a); the movie shows the whole slices set sequentially. (c) A longitudinal cross-sectional image rebuilt from 3-D image volume; the image size was $6 \mathrm{~mm}$ by $1.3 \mathrm{~mm}$. (d) The histology image at corresponding position of (c). (e=epithelium; lp=lamina propria; $\quad c=$ cartilage; $\mathrm{sm}=$ submucosa; $\mathrm{tm}=$ trachealis muscle.) (Image taken from ref[140])
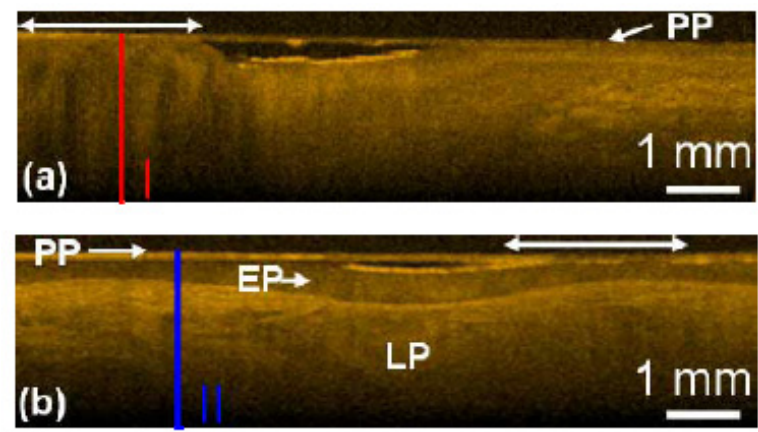

Fig. A5. SS-OCT scanning images of the cancerous (a) and normal oral mucosal (b) tissues indicated by the red and blue arrows, respectively, in Fig. 2. In the cancerous tissue, the boundary between EP and LP layers disappeared in the whole B-mode scan range. The red and blue vertical lines (labeled as I and II, respectively) and horizontal white arrows indicate the ranges of detailed analyses later. PP: plastic plate. (Image taken from ref[152]) 
(4) Biological samples

Tadpole is commonly used for developmental biology sample for investigating organogenesis [124]. OCT image reveals inner micro-structure of organs.
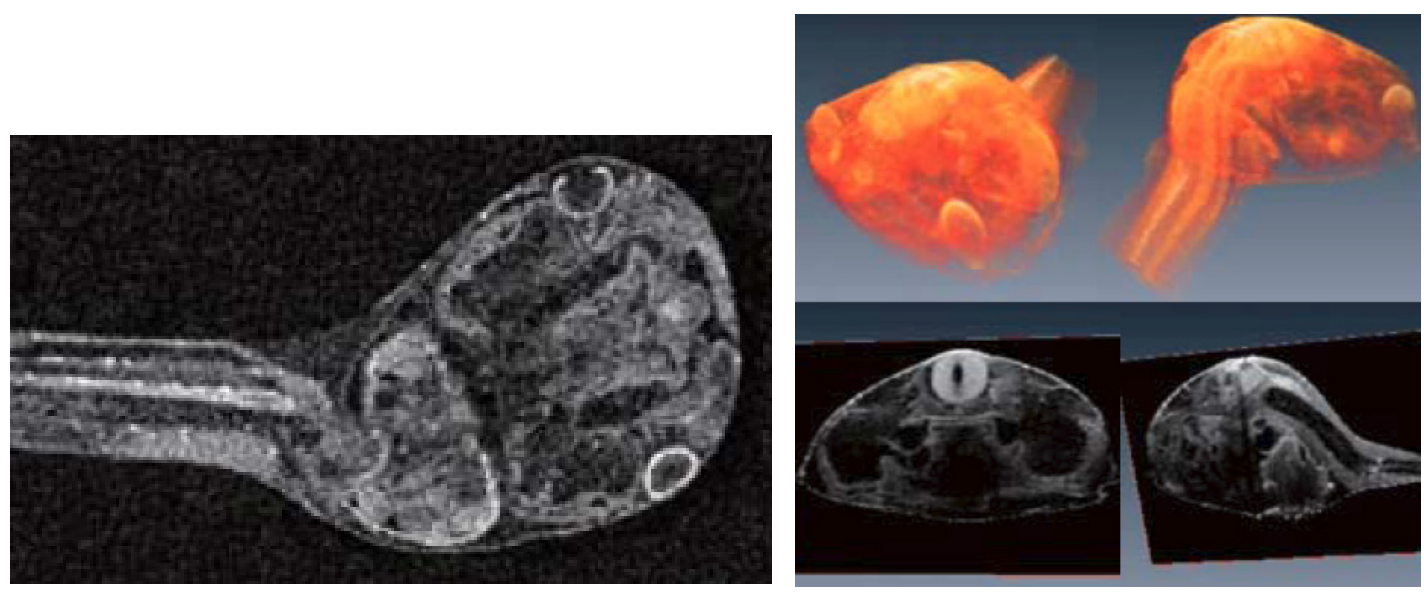

Figure A6. OCT image of Tadpole (Xenopus laevis, Albino, 2 weeks, $2 \mathrm{~mm}$ in size of head)

Observation of embryo implies the organogenesis, which is the origin of organs from single cell level.
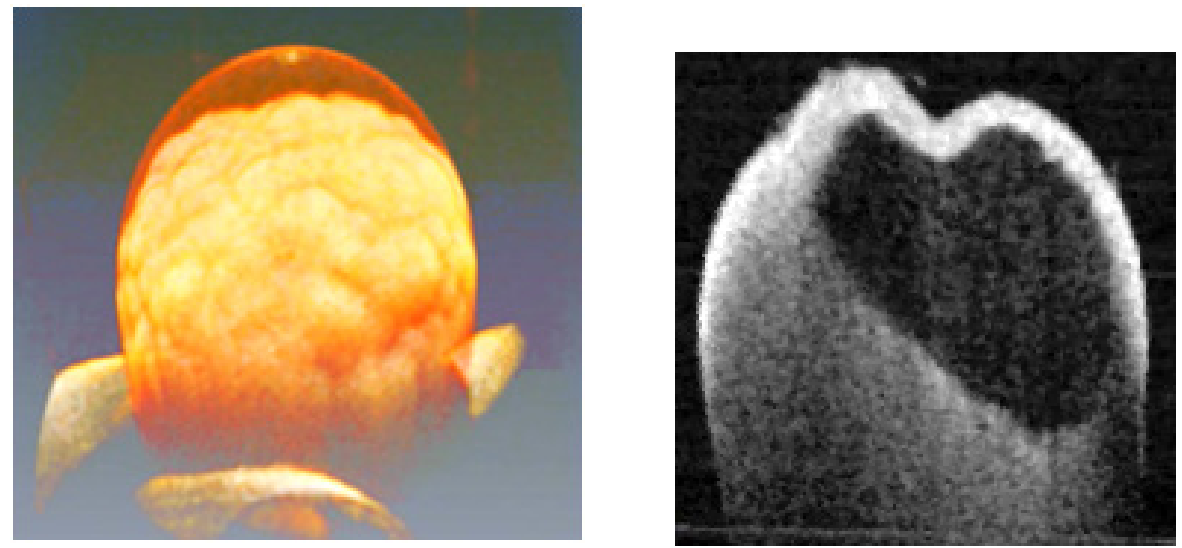

Figure A7 OCT image of Embryo of Tadpole after 24 hrs from first cell division 


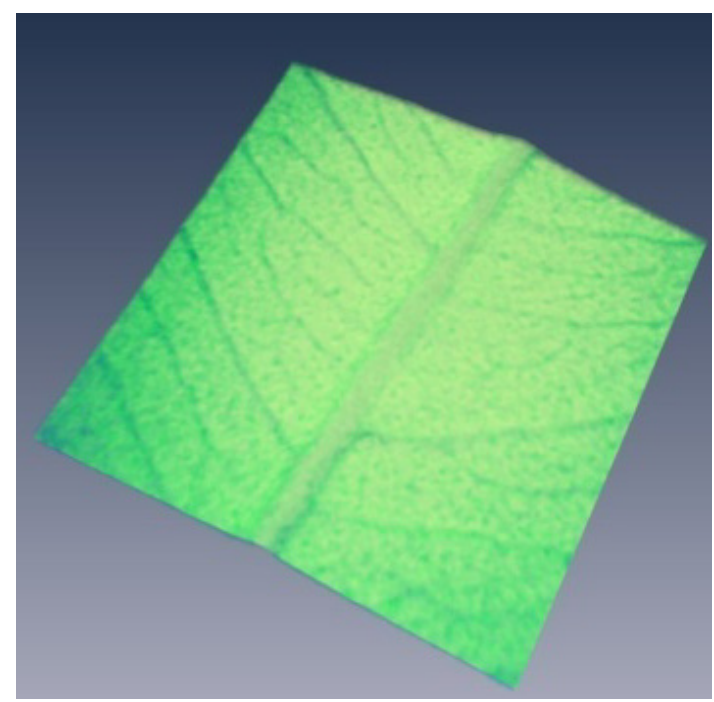

Figure A8. OCT image of leaf
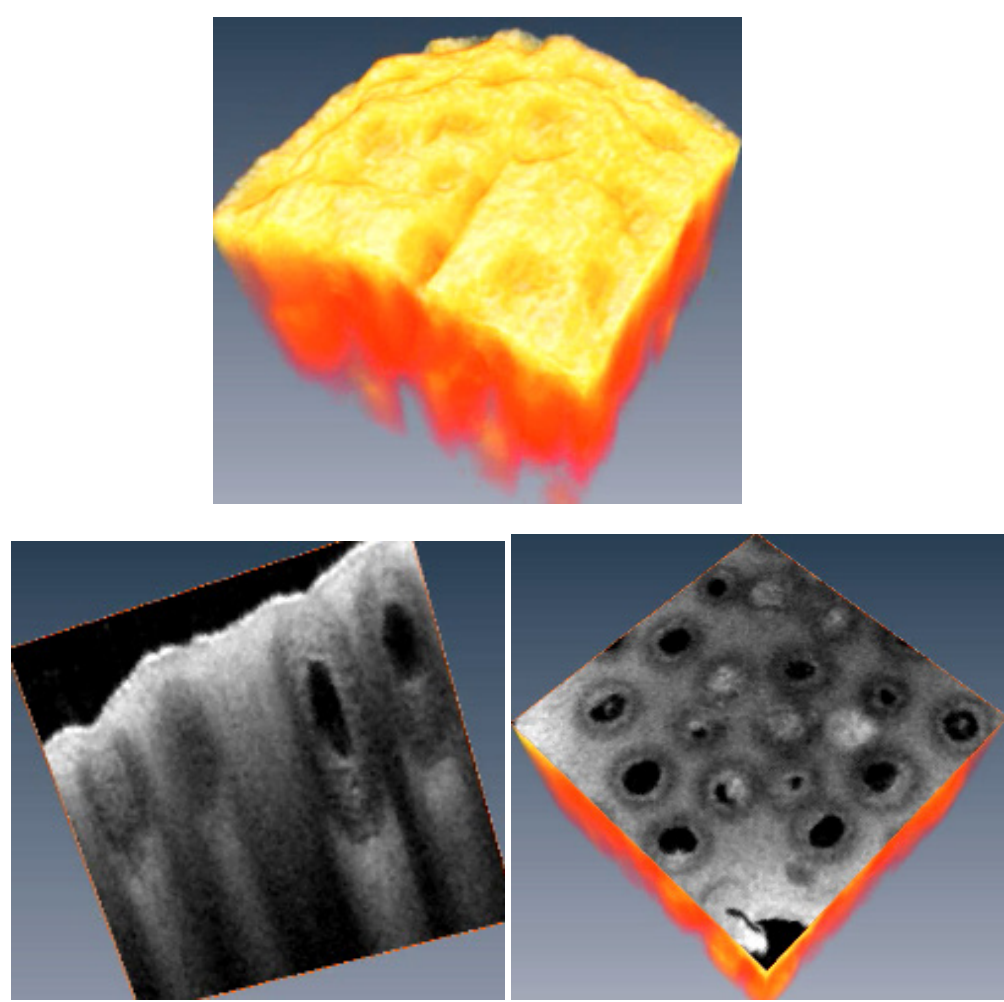

Figure A9 Orange skin 
(5) Industrial/material inspection

A. Semiconductor devices

Silicon wafers and most of dielectric materials are transparent at infra-red range over $1 \mu \mathrm{m}$. As long as the device is not coated with metal (e.x. gold, or aluminum), OCT imaging is possible. One concern is that multiple reflections from the strong reflective surfaces that cause multiple aliasing on the image that make it difficult to distinguish the actual structure from the image. One way to avoid this problem is simply tilting the sample with respect to the probe beam. Additional adjustment of contract enhances the image clarity suppressing the aliasing noise. The OCT image of the MEMS pressure sensor was captured. Slice images were taken at different planes through the micromirror device. Microscale features, such as top diaphragm, membranes, and cavity in between these, and bottom electrode are clearly visible in the stereo and the en face images. Scaling of the device is limited by minimum thickness of the layer which is $12 \mu \mathrm{m}$ divided by refractive index of silicon $(\mathrm{n}=3.5) ; 3.4 \mu \mathrm{m}$ which is minimum resolvable scale in silicon, $12 \mu \mathrm{m}$ in air if scaling the cavity. Scaling accuracy is repeatability of the measurement system which exceeds about submicron level. Furthermore, functional imaging such as monitoring inner stress of material can be realized by implementing phase-resolved approaches [125].

Below MEMS device is imaged with SS-OCT using $1.3 \mu \mathrm{m}$ source at $20 \mathrm{kHz}$ swept rate.
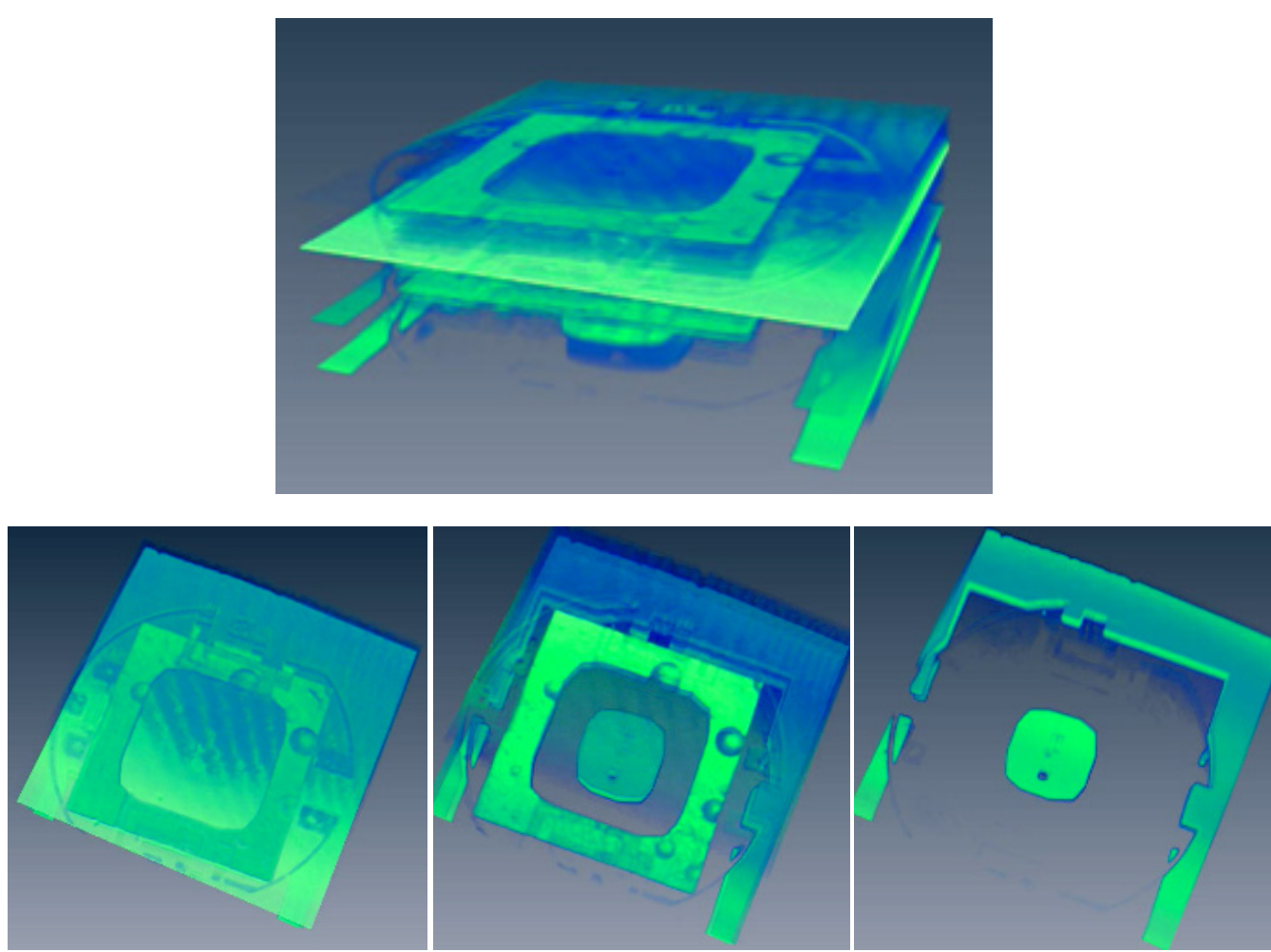

Figure A10.Three dimensional structure of MEMS pressure sensor

(a)Stereo view, (b) Fly-through from top layer (diaphragm), (b) Membrane, (c) bottom anchor 
B. Plastic material
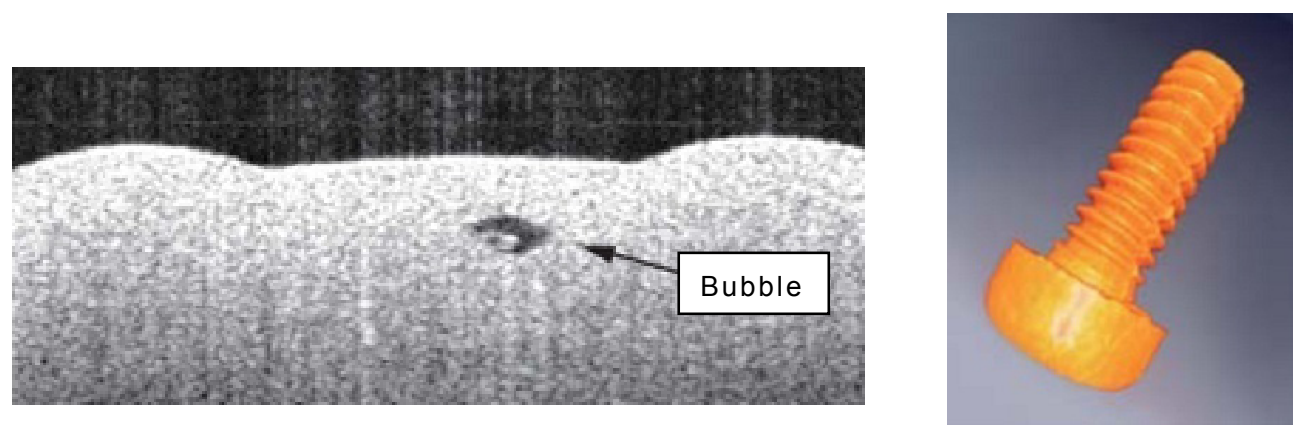

Figure A11 Plastic molding

This is colored plastic. As long as the coloring dye is not absorptive like in black, infrared light can penetrate a certain degree to realize cross sectioning. Left picture shows the bubble trapped during the molding process. OCT can be used as inspection system or monitoring tool for process or quality control.

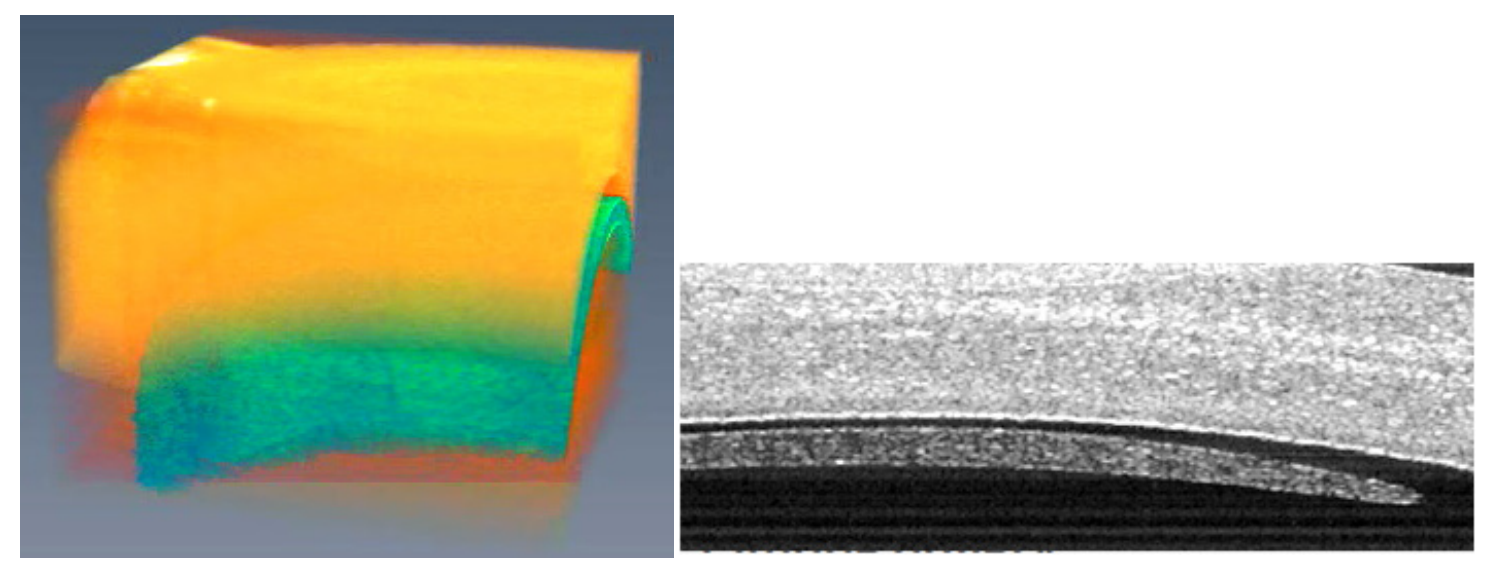

Figure A12 OCT image of disposable contact lens floating inside the container case

Figure A12 shows image of contact lens. This also promises the surface or contour profiling application in numerous kinds of industrial hardware.

C. Coating material

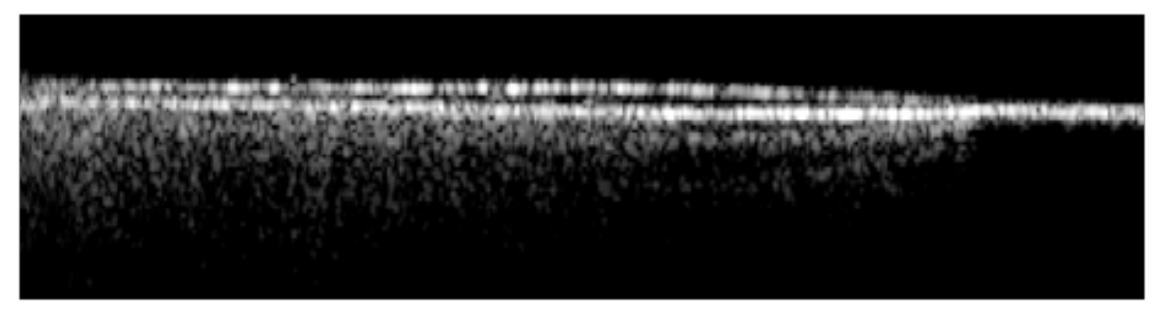

Figure A13 Paint on the metal 
Although it is not possible to image the cross section the metal sample, thin, transmissive material in infra-red band like Alumite (ceramic) can be penetrated so that thickness measurement is possible. Conventional thickness measurement based on white light interferometry is limited to the smooth surface. But OCT can scale and image the boundary of these relatively rough coating to estimate the thickness or coating strength. Other functional OCT such as phase-sensitive OCT also enables accurate measurement of translation or dimension in nanometer order comparable to that of confocal microscopy. 
(6) Commercialized medical applications

A. Michelson Diagnostics Incorporated.(UK)

MDL incorporate $1.3 \mu \mathrm{m}$ source with $160 \mathrm{~nm}$ wideband sweep range, at $10 \mathrm{kHz}$ swept rate that we designed and manufacture. Application is ex-vivo, in-vivo diagnosis of cancer.

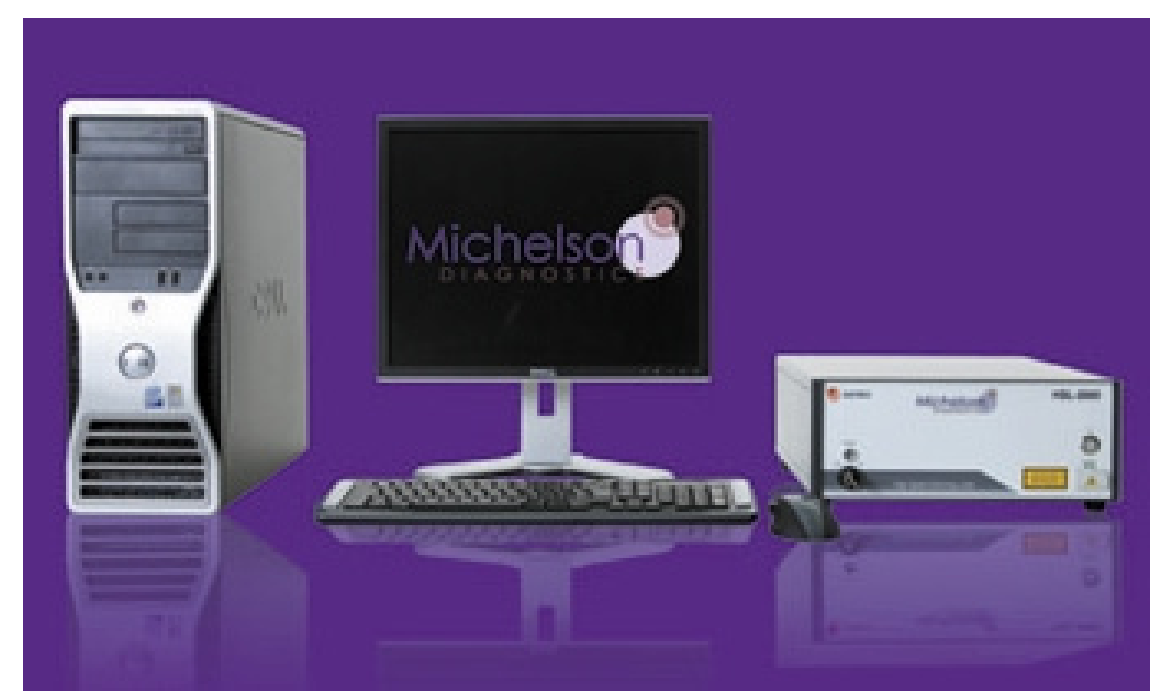

Figure A14. SS-OCT system from MDL

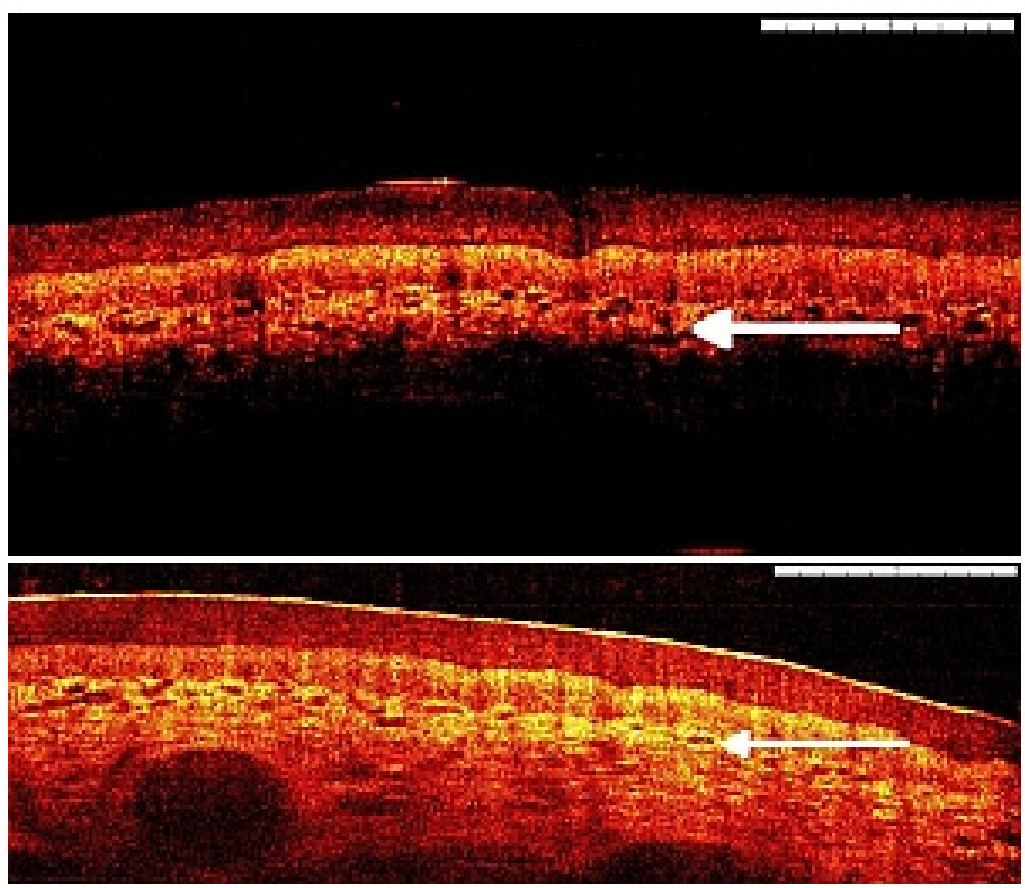

Figure A15. SS-OCT image of human skin with $1 \mu \mathrm{m}$ source (top) and $1.3 \mu \mathrm{m}$ source(bottom) (In courtesy of MDL, http://www.mdl-1td.co.uk) 
B. Tomey Corporation(Japan)
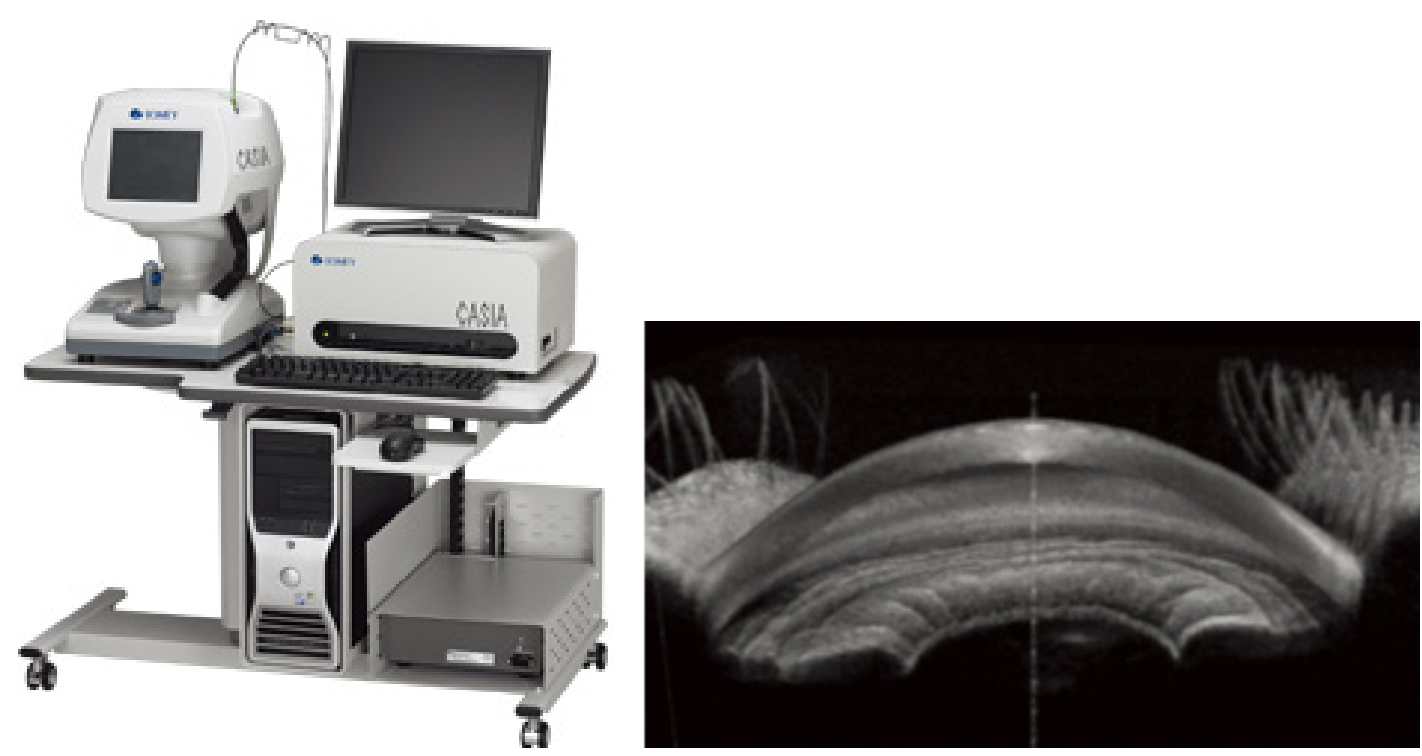

-開塞隅角緑内障

隅角が開塞していることかわかります。

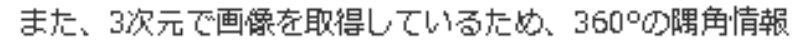
を得ることが可能です。

- LASIK䝻

டASIKのフラップ描出されています。

非接触て撮影するため、術直啳ても摄影可能です。
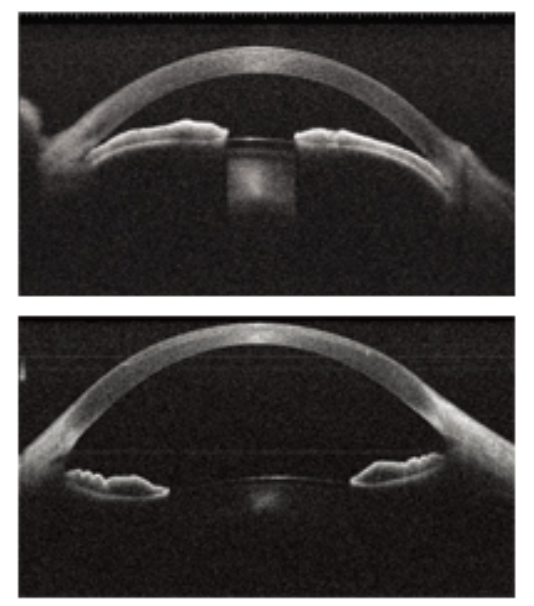

\section{-涯過胞}

(線維柱帯切] 除術後)

承榢は黒く描出されるます。

非接触て撮影するため、術直後でも撮影可能です。

\section{- 円錐角膜}

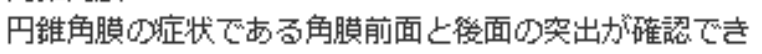
ます。
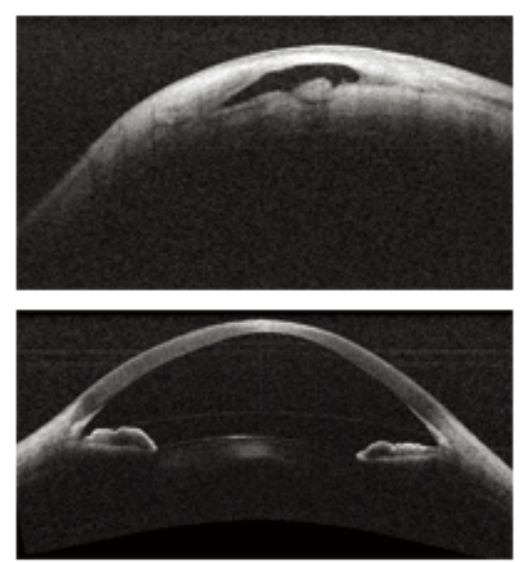

Figure A16. SS-OCT system for anterior chamber diagnostics (SS-1000) (In courtesy of TOMEY CO.)

(Web site catalogue from http://www.tomey.co.jp/tomey_corp/) 
Table A1. Reference of the work that uses our swept-source in SS-OCT for various applications

\begin{tabular}{|c|c|c|c|c|}
\hline Author(s) & Institution & Application & \begin{tabular}{|l|}
$\begin{array}{l}\text { Swept source main } \\
\text { characteristics }\end{array}$ \\
\end{tabular} & Ref. \\
\hline Y.Yasuno, M.Miura, et al. & Tsukuba Univ. & Ophthalmology (Retina) & $1.0 \mathrm{um}, 28 \mathrm{kHz}$ & 139 \\
\hline Jianping Su, et al & UC Irvine & Endoscopy & $1.3 \mathrm{um} 20 \mathrm{kHz}$ & 140 \\
\hline Yoshiaki Yasuno et al & Tsukuba Univ. & Ophthalmology (Retina) & $1.0 \mathrm{um}, 28 \mathrm{kHz}$ & 141 \\
\hline Jianping Su, et al & UC Irvine & Endoscopy & $1.3 \mathrm{um} 20 \mathrm{kHz}$ & 142 \\
\hline Yoshiaki Yasuno et al & Tsukuba Univ. & $\begin{array}{l}\text { Ophthalmology(Anterior } \\
\text { segment) }\end{array}$ & $1.3 \mathrm{um} 20 \mathrm{kHz}$ & 143 \\
\hline Masahiro Yamanari, et al & Tsukuba Univ. & Ophthalmology (Retina) & $1.0 \mathrm{um} 20 \mathrm{kHz}$ & 144 \\
\hline R K Wang et al & Oregon Health Univ. & General & $1.55 \mathrm{um} 20 \mathrm{kHz}$ & 145 \\
\hline Masahiro Yamanari et al. & Tsukuba Univ. & Ophthalmology (Retina) & $1.3 \mathrm{um} 20 \mathrm{kHz}$ & 146 \\
\hline Jun Zhang, et al & UC Irvine & Endoscopy & $1.3 \mathrm{um} 20 \mathrm{kHz}$ & 147 \\
\hline Jon Holmes, et al & Michelson Diagnostics & Skin, Cancer & $\begin{array}{l}1.3 \mathrm{um} \text { wide } 160 \mathrm{~nm}, \\
10 \mathrm{kHz}\end{array}$ & 148 \\
\hline Youxin Mao et al. & $\begin{array}{l}\text { National Research } \\
\text { Coucil, Canada }\end{array}$ & General & $1.3 \mathrm{um} 20 \mathrm{kHz}$ & 149 \\
\hline Kumar, K, et al & Univ. of Texas & Endoscopy & $1.3 \mathrm{um} 20 \mathrm{kHz}$ & 150 \\
\hline Meng-Tsan, et al & National Taiwan Univ. & Oral cancer & $1.3 \mathrm{um} 20 \mathrm{kHz}$ & 151 \\
\hline Meng-Tsan Tsai, et al. & National Taiwan Univ. & Oral cancer & $1.3 \mathrm{um} 20 \mathrm{kHz}$ & 152 \\
\hline
\end{tabular}




\section{Acknowledgments}

The author would like to express his sincere thanks to Professor Yasuo Kokubun, Yokohama National University for his kind guidance and continuous encouragement throughout the course of this study. The author would like to thank Professor Toshihiko Baba of Yokohama National University for valuable comments and reviews to his study. During the course work of $\mathrm{PhD}$ program, he gained his attitude toward academic and scientific research process.

The author would like to thank Dr. Daikou Tei, President and CEO of santec corporation for letting him pursue the degree while he was working in the company at the same time and also for supporting him financially for the tuition.

The author would like to thank Naoyuki Mekada, R\&D director and his manager in santec corporation for his supportive management in the project and valuable advises. He also thanks his colleagues, Atsushi Morosawa and Tooru Sakai for helping him from the start of his project. He especially thanks Morosawa and Takuya Suzuki for programming the source code for SS-OCT algorithm from the scratch. He also thanks the other colleagues, Yukiya Mikuni, Hiroshi Moriyama, Tatsu Yo, Koki Totsuka in his research group in santec who supported his work in many engineering design and fabrication of the prototypes as well as experiments. He also thanks Dr. Jonathan W. Evans of santec USA corporation for checking his English grammar in the research papers.

The author also would like to thank Optoelectronic Industry and Technology Development Association (OITDA) for providing a grant on his project in the year 2006 and 2007.

The author would like to thank Professor Hiroshi Toshiyoshi for the collaboration in MEMS development for the probe. He also thanks Muneki Nakada, PhD course student for helping him develop and discussing new idea of MEMS structure. The author thanks Dr.T.Makita and K.Shiba at NEC for providing us with samples of photovoltaic detector for prototyping the module.

The author also wants to acknowledge extensive discussion with Dr. Yoshiaki Yasuno of Tsukuba University regarding theoretical and experimental results associating with clinical relevance and his knowledge resources. 
He also would like to express his gratitude to Dr.Yasunori Sumi and Dr. Nobuyoshi Ozawa of National Hospital for Geriatric Medicine for their help in clinical studies in imaging oral tissue with developed SS-OCT.

The author wants to thank all the member of Kokubun Laboratory for sharing discussion with his study.

He thanks Technician Eiji Sato, Yokohama National University, for support.

He thanks all the professors, assistant and supports of the department of Electrical and Computer Engineering, Yokohama National University.

He thanks all the OCT researchers in the world who provided with valuable comments and feedbacks to his work. 


\section{REFERENCES}

1. S.T. Sanders, J.A. Baldwin, T.P. Jenkins, D.S. Baer, and R.K. Hanson, "Diode-laser sensor for monitoring multiple combustion parameters in pulse detonation engines," P. Combust. Inst. 28, 587-594 (2000)

2. L. A. Kranendonk, R. Huber, J. G. Fujimoto, and S. T. Sanders, "Wavelength-agile H2O absorption spectrometer for thermometry of general combustion gases," Proceedings of the Combustion Institute 31,783-790 (2007)

3. S.T. Sanders, J. Wang, J.B. Jeffries, and R.K. Hanson, "Diode-laser absorption sensor for line-of-sight gas temperature distributions," Applied Optics 40, 4404-4415 (2001)

4. J. Wang, S.T. Sanders, J.B. Jeffries, and R.K. Hanson, "Oxygen measurements at high pressures with vertical cavity surface-emitting lasers," Applied Physics. B 72, 865-872 (2001)

5. L. A. Kranendonk, X. An, A. W. Caswell, R. E. Herold, S. T. Sanders, R. Huber, J. G. Fujimoto, Y. Okura, and Y. Urata, "High speed engine gas thermometry by Fourier-domain mode-locked laser absorption spectroscopy," Optics Express 15, 15115-15128 (2007).

6. S.H.Yun, D.J.Richardson, B.Y.Kim, "Interrogation of fiber grating sensor arrays with a wavelength-swept fiber laser, " Optics Letter 23, 843-845 (1998)

7. D. Huang, E. A. Swanson, C. P. Lin, J. S. Schuman, W. G. Stinson, W. Chang, M. R. Hee, T. Flotte, K.Gregory, C. A. Puliafito, and J. G. Fujimoto, "Optical coherence tomography," Science 254, 1178-1181(1991)

8. M. E. Brezinski, G. J. Tearney, B. E. Bouma, J. A. Izatt, J. M. Schmitt, A. Knuttle, and R. F. Knuttle, "Measurement of optical properties of biological tissues by low coherence interferometry,” Applied Optics 32 6032-6042 (1993) 
9. J. G. Fujimoto, M. E. Brezinski, G. T. Tearney, S. A. Boppart, B. E. Bouma, M. R. Hee, J. F. Southern, and E. A. Swanson, "Biomedical imaging and optical biopsy using optical coherence tomography," Nature Medicine 1, 970-972 (1995)

10. M. R. Hee, E. A. Swanson, J. F. Southern, and J. G. Fujimoto, "Optical coherence tomography for optical biopsy-properties and demonstration of vascular pathology," Circulation 93, 1206-1213 (1996).

11. G. J. Tearney, M. E. Brezinski, B. E. Bouma, S. A. Boppart, C. Pitris, J. F. Southern, and J. G. Fujimoto, "In vivo endoscopic optical biopsy with optical coherence tomography," Science 276, 2037-2039 (1997)

12. M. E. Brezinski and J. G. Fujimoto, "Optical coherence tomography: high-resolution imaging in nontransparent tissue", IEEE J. Selected Topic of Quantum Electron 5, 1185-1192 (1999)

13. S. R. Chinn, E. A. Swanson, and J. G. Fujimoto, "Optical coherence tomography using a frequency-tunable optical source, " Optics Letter 22, 340-342 (1997)

14. B. Golubovic, B. E. Bouma, G. J. Tearney, and J. G. Fujimoto, "Optical frequency-domain reflectometry using rapid wavelength tuning of a $\mathrm{Cr} / \mathrm{sup} 4+/$ :forsterite laser, " Optics. Letter 22, 1704-1706 (1997)

15. A. F. Fercher, C. K. Hitzenberger, G. Kamp, and S. Y. Elzaiat, "Measurement Of intraocular distances by backscattering spectral interferometry, " Optical Communication 117, 43-48 (1995)

16. A.Wax, C. H. Yang, and J. A. Izatt, "Fourier-domain low-coherence interferometry for light-scattering spectroscopy," Optics Letter 28, 1230-1232 (2003)

17. G. Häusler, and M. W. Lindner, "Coherence radar" and "spectral radar"-new tools for dermatological diagnosis," J. Biomedical Optics 3, 21-31 (1998).

18. J. F. de Boer, B. Cense, B. H. Park, M. C. Pierce, G. J. Tearney, and B. E. Bouma, "Improved signal-to-noise ratio in spectral-domain compared with time-domain optical coherence tomography," Opt. Lett. 28, 2067-2069 (2003). 
19. R. Leitgeb, C. K. Hitzenberger, and A. F. Fercher, "Performance of fourier domain vs. time domain optical coherence tomography," Optics Express 11, 889-894 (2003).

20. S.H. Yun, G. J. Tearney, J. F. de Boer, and B. E. Bouma, "Pulsed-source and swept-source spectral-domain optical coherence tomography with reduced motion artifacts, " Optics Express 12, 5614 (2004)

21. S. H. Yun, G. J. Tearney, J. F. de Boer, and B. E. Bouma, "Motion artifacts in optical coherence tomography with frequency-domain ranging," Optics Express 12, 2977-2998 (2004)

22. M.A. Choma, M.V. Sarunic, C.Yang, and J.Izatt, "Sensitivity advantage of swept source and Fourier domain optical coherence tomography, " Optics Express 11, 2183-2189 (2003)

23. Wolfgang Drexler and James G. Fujimoto, Optical Coherence Tomography: Technology and Applications, (springer 2008)

24. S.H.Yun, C.Boudoux, M.C.Pierce, G.J.Tearney, J.F.de Boer, and B.E.Bouma, "Extended-cavity semiconductor wavelength-swept laser for biomedical imaging, " IEEE Photonics Technology Letter 16, 293-295 (2004)

25. Michael A. Choma, Kevin Hsu, and Joseph A. Izatt, "Swept source optical coherence tomography using an all-fiber 1300-nm ring laser source, " J. Biomedical Optics 10, 044009 $(2005)$

26. Timothy R. Hillman and David D. Sampson, "The effect of water dispersion and absorption on axial resolution in ultrahigh-resolution optical coherence tomography," Optics Express 13, $1860(2005)$

27. T. L. Troy and S. N. Thennadil, "Optical properties of human skin in the near infrared wavelength range of 1000 to $2200 \mathrm{~nm}$, " J. Biomed. Opt. 6, 167-176 (2001)

28. G. M. Hale and M. R. Querry, "Optical constants of water in the $200 \mathrm{~nm}$ to $200 \mu \mathrm{m}$ wavelength region," Applied Optics 12, 555-563 (1973) 
29. L. Kou, D. Labrie, and P. Chylek, "Refractive indices of water and ice in the $0.65-2.5$ $\mu \mathrm{m}$ spectral range, " Applied Optics 32, 3531-3540 (1993)

30. J. F. de Boer, B. H. Park, E. C. W. Lee, R. Yelin, and S. H. Yun "Optical frequency domain imaging with a rapidly swept laser in the 815-870 nm range, Optics Express 14, 5937 (2006)

31. V. Srinivasan, R. Huber, I. Gorczynska, J. Fujimoto, J. Jiang, P. Reisen, and A. Cable, "High-speed, high resolution Optical Coherence Tomography retinal imaging with a frequency-swept laser at $850 \mathrm{~nm}$, " Optics Letter 32, 361-363 (2007)

32. Frederik D. Nielsen, Lars ThraneJohn F. Black, Anders Bjarklev, Peter E. Andersen "Swept wavelength source in the $1 \mu \mathrm{m}$ Range, " Optics Express 13, 4096 (2005)

33. R. Huber, D. C. Adler, V. J. Srinivasan, and J. G. Fujimoto, "Fourier domain mode locking at $1050 \mathrm{~nm}$ for ultra-high-speed optical coherence tomography of the human retina at 236,000 axial scans per second," Optics Letter 32, 2049-2051 (2007).

34. Jun Zhang, a_ Qiang Wang, Bin Rao, and Zhongping Chen "Swept laser source at $1 \mu \mathrm{m}$ for Fourier domain optical coherence tomography, " Applied Physics Letters 89, 073901 (2006)

35. A. Unterhuber, B. Považay, B. Hermann, H. Sattmann, A. Chavez-Pirson, and W. Drexler, "In vivo retinal optical coherence tomography at $1040 \mathrm{~nm}$ - enhanced penetration into the choroid," Optics Express 13, 3252-3258 (2005)

36. B. E. Bouma, L. E. Nelson, G. J. Tearney, D. J. Jones, M. E. Brezinski, and J. G. Fujimoto, "Optical coherence tomographic imaging of human tissue at $1.55 \mu \mathrm{m}$ and $1.81 \mu \mathrm{m}$ using Er- and Tm-doped fiber sources, " J. Biomedical Optics 3, 76-79 (1998)

37. U. Sharma, E. W. Chang, and S. H. Yun, "Long-wavelength optical coherence tomography at $1.7 \mu \mathrm{m}$ for enhanced imaging depth," Opt. Express 16, 19712-19723 (2008)

38. J Su, J Zhang, Z.P.Chen, "In-vivo three-dimensional microelectromechanical endoscopic swept source optical coherence tomography, " Optics Express 15 10390-10396 (2007) 
39. Y.Yasuno, YJ Hong, S.Makita,M.Yamanari, M.Akiba, M.Miura,T.Yatagai, "In vivo high-contrast imaging of deep posterior eye by $1-\mu \mathrm{m}$ swept source optical coherence tomography and scattering optical coherence angiography," Optics Express 15, 6121-6139 (2007)

40. Tearney, G. J., Boppart, S. A., Nouma, B. E., et al. "Scanning Single-mode fiber optics catheter-endoscope for optical coherence tomography, " Optics Letter, 21, 543-545 (1996)

41. Y. Yasuno, V. D. Madjarova, S. Makita, M. Akiba, A. Morosawa, C. Chong, T. Sakai, K. P. Chan, M.Itoh, and T. Yatagai, " Three-dimensional and high-speed swept-source optical coherence tomography for in vivo investigation of human anterior eye segments, "Optics Express 13, 10652-10664 (2005)

42. B. W. Colston Jr, M. J. Everett, U. S. Sathyam, L. B. DaSilva, and L. L. Otis, " Imaging of the oral cavity using optical coherence tomography, " Assessment of Oral Health, Monograms in Oral Science, R. V.Faller, ed., (Basel, Karger, 2000), vol 17, 32-55

43. A Takabayashi, K. Takada, K.Hashimoto, N.Doi, M.Tomabechi, S.Nakazawa, T.Morito, " Widely (132 nm) wavelength tunable laser using a semiconductor optical amplifier and an acousto-optic tunable filter, " Electronics Lett., Vol. 40, No. 19, 1187-1188 (2004)

44. Y. Deki, M. Takahashi, K. Suzuki, M. Ishizaka, S. Takaesu, M. Horie, K. Sato, K. Kudo, and H. Yamazaki, "A 160-nm-wavelength tunable laser using a waveguide double-ring resonator, ". OECC'2005, Paper 7F3-3, July 2005

45. H. Oohashi, Y. Shibata, H. Ishii, Y. Kawaguchi, Y. Kondo, Y. Yoshikuni, and Y.Tohmori, "46.9-nm wavelength-selectable. arrayed DFB lasers with integrated MMI coupler and SOA,". IPRM '2001, FBI-2, pp. 575-578

46. P. J. Rigole, S. Nilsson, L. Bäckbom, T. Klinga, J. Wallin, B. Stålnacke,. E. Berglind, and B. Stoltz, "114-nm wavelength tuning range of a grating assisted codirectional coupler laser with a super structure grating distributed Bragg reflector, " IEEE Photon. Technol. Lett., vol. 7, pp. 697-699, (1995)

47. S.H.Yun, G.J.Tearney, J.F.de Boer,N.Iftimia and B.E.Bouma, " High-speed optical frequency-domain imaging, " Optics Express 11, 2953-2963, (2003) 
48. C.Chong, A.Morosawa, T.Sakai, " High speed wavelength-swept laser source with simple configuration for optical coherence tomography, " Proc. of SPIE -- Volume 6627, Optical Coherence Tomography and Coherence Techniques III, 662705, Jun. 26, 2007

49. R.Huber, M.Wojtkowski, K.Taira, and J.G.Fujimoto, " Amplified, frequency swept lasers for frequency domain reflectometry and OCT imaging: design and scaling principles, " Optics Express 13, 3513-3528 (2005)

50. R. Huber, M. Wojtkowski, and J. G. Fujimoto, "Fourier Domain Mode Locking (FDML): A new laser operating regime and applications for optical coherence tomography, "Optics Express 14, 3225-3237 (2006)

51. S.H.Yun, C.Boudoux G.J.Tearney, and B.E.Bouma, "High-speed wavelength-swept semiconductor laser with a polygon-scanner-based wavelength filter, " Optics Letter 28 , $1981-1983$ (2003)

52. W. Y. Oh, S. H. Yun, G. J. Tearney, and B. E. Bouma, "Wide Tuning Range Wavelength-Swept Laser With Two Semiconductor Optical Amplifiers" IEEE Photonics Technology Letters, VOL. 17, NO. 3, MARCH 2005

53. R.Huber, M.Wojtkowski, J.Fujimoto, J.Y.Jiang, A.E.Cable, "Three-dimensional and C-mode OCT imaging with a compact, frequency swept laser source at 1300nm, " Optics Express 13, 10523-10538(2005)

54. W. Y. Oh, S. H. Yun, G. J. Tearney, and B. E. Bouma, "1 $15 \mathrm{kHz}$ tuning repetition rate ultrahigh-speed wavelength-swept semiconductor laser, " Optics Letters 30, 3159-3161 $(2005)$

55. S. M. R. Motaghian Nezam, "High-speed polygon-scanner-based wavelength swept laser source in the telescope-less configurations with application in optical coherence tomography" Optics Letters 33, 1741-1743 (2008)

56. S. H. Yun, G. J. Tearney, J. F. de Boer, and B. E. Bouma, " "Removing the depth-degeneracy in optical frequency domain imaging with frequency shifting, " Optics Express 12, 4822-4828, 2004 
57. J. Zhang, J. S. Nelson, and Z. P. Chen, "Removal of a mirror image and enhancement of the signal-to-noise ratio in Fourier-domain optical coherence tomography using an electro-optic phase modulator, " Optics Letter 30, 147-149 (2005)

58. A. M. Davis, M. A. Choma, and J. A. Izatt, "Heterodyne sweptsource optical coherence tomography for complete complex conjugate ambiguity removal, " J Biomedical Optics 10, 064005 (2005).

59. M.V. Sarunic, M.A. Choma, C.H. Yang, and J.A. Izatt, "Instantaneous complex conjugate resolved spectral domain and swept-source OCT using $3 \times 3$ fiber couplers," Optics Express 13, 957-967 (2005)

60. A.Bilenca, S. H. Yun, G. J. Tearney, and B. E. Bouma, "Numerical study of wavelength-swept semiconductor ring lasers: the role of refractiveindex nonlinearities in semiconductor optical amplifiers and implications for biomedical imaging applications," Optics Letters 31 (2006)

61. G.P.Agrawal, "Population pulsations and nondegenerate four-wave mixing in semiconductor lasers and amplifiers," J.OSA B, vol.5, 147-159, (1988)

62. K.Inoue, T.Mukai,and T.Saitou, "Nearly degenerate four-wave mixing in a traveling -wave semiconductor laser amplifier," Applied Physics Letters, 151, 1051-1053 (1987)

63. R.Tripathi, N.Nassif, J.Stuart Nelson, B.H.Park, and J.F.de Boer, "Spectral shaping for non-Gaussian source spectra in optical coherence tomography," Optics Letter 27 (2002)

64. S.H.Yun, D. J. Richardson, D. O. Culverhouse, and B. Y. Kim "Wavelength-Swept Fiber Laser with Frequency Shifted Feedback and Resonantly Swept Intra-Cavity Acoustooptic Tunable Filter, " IEEE J. of Selected Topics In Quantum Electronics 3, 1087-1076 (1997)

65. R. Huber, D. C. Adler, and J. G. Fujimoto, "Buffered Fourier domain mode locking: unidirectional swept laser sources for optical coherence tomography imaging at 370,000 lines/s," Optics Letter 31, 2975-2977 (2006)

66. S. W. Huang, A. D. Aguirre, R. A. Huber, D. C. Adler, and J. G. Fujimoto, "Swept 
source optical coherence microscopy using a Fourier domain mode-locked laser," Opt. Express 15, 6210-6217 (2007).

67. D. C. Adler, R. Huber, and J. G. Fujimoto, "Phase-sensitive optical coherence tomography at up to 370,000 lines per second using buffered Fourier domain mode-locked lasers," Optics Letter 32, 626-628 (2007)

68. W. Jenkins, D. C. Adler, M. Gargesha, R. Huber, F. Rothenberg, J. Belding, M. Watanabe, D. L. Wilson, J. G. Fujimoto and A. M. Rollins "Ultrahigh-speed optical coherence tomography imaging and visualization of the embryonic avian heart using a buffered Fourier Domain Mode Locked laser, " Optics Express 15, 6251(2007)

69. W. R. Trutna, Jr., and L. F. Stokes, "Continuously Tuned External Cavity Semiconductor Laser," Journal of Lightwave Technology 11, 1279- 1286 (1993)

70. C. Pitris, C. Jesser, S. A. Boppart, D. Stamper, M. E. Brezinski, and J. G. Fujimoto, "Feasibility of optical coherence tomographyfor high resolution imaging of human gastrointestinal tract malignancies, " J.Gastroenterol. 35, 87-92 (2000).

71. M. Wojtkowski, A. Kowalczyk, R. Leitgeb, and A. F. Fercher, "Full range complex spectral optical coherence tomography technique in eye imaging, " Optics Letter 27, 1415-1417 (2002).

72. L.A. Kranendonk, R.J. Bartula, and S.T. Sanders, "Modeless operation of a wavelength -agile laser by high-speed cavity length changes," Optics Express 13, 1498-1507 (2005).

73. RF.Spaide, H.Koizumi, MC.Pozonni, "Enhanced Depth Imaging Spectral-Domain Optical Coherence Tomography," Am J Ophthalmol. 2008 Jul 16. [Epub ahead of print]

74. E. C. Lee, J. F. de Boer, M. Mujat, H. Lim, and S. H. Yun, "In vivo optical frequency domain imaging of human retina and choroid," Optics Express 14, 4403-4411 (2006)

75. F. Lexer, C.K. Hitzenberger, A.F. Fercher, and M. Kulhavy, "Wavelength-tuning interferometry of intraocular distances," Applied Optics 36, 6548-6553 (1997)

76. C.Chong, T.Suzuki, A.Morosawa, T.Sakai, N.Mekada, "Coherence Length Improvement 
by Spectral Narrowed Wavelength-Swept Laser with Quasi-Phase Continuous Tuning" Biomedical Optics topical meeting OSA 06, St.Petersberg, Florida, 2008.3.21

77. T.Olsen, M.Thorwest "Calibration of axial length measurements with the Zeiss IOL Master", Journal of cataract and refractive surgery, vol. 31, no7, pp. 1345-1350 (2005)

78. I. Kielhorn, "Clinical assessment of the Zeiss IOLMaster," Journal of Cataract \& Refractive Surgery, Volume 29, Issue 3, Pages $518-522$

79. "Diode Lasers and Photonic Integrated Circuits" L.A.Coldren, S.W.Corzine, Wiley Series in Microwave and Optical Engineering, NY(1995)

80. R. A. Leitgeb, W. Drexler, A. Unterhuber, B. Hermann,T. Bajraszewski, T. Le, A. Stingl, and A. F. Fercher, "Ultrahighresolution Fourier domain optical coherence tomography," Optics Express 12, 2156-2165 (2004)

81. H.Lim, J.F.de Boer, B.H.Park, E.C.W.Lee, R.Yelin, and S.H.Yun "Optical frequency domain imaging with a rapidly swept laser in the 815-870nm range”, Opt Express Vol. 14, No. 13, (2006)

82. M. Fukuda, Reliability and Degradation of Semiconductor Lasers and LEDs, (Artech House Publishers,(1991).

83. B. M. Green, K. K. Chu, E. M. Chumbes, J. A. Smart, J. R. Shealy, and L. F. Eastman, "The effect of surface passivation on the microwave characteristicsof undoped AlGaN/GaN HEMTs,” IEEE Electron.Dev. Lett. 21, 268-270 (2000).

84. C.Chong, A.Morosawa, Tooru Sakai, "High-speed wavelength-swept laser source with high-linearity sweep for optical coherence tomography," IEEE Journal of Selected Topics on Quantum Electronics, 14, 235-242 (2008)

85. E.Brinkmeyer, R.Ulrich, "High-resolution OCDR in dispersive waveguides, " Electron. Lett 26, 413-414 (1990)

86. K. Takada, K. Yukimatsu, M. Kobayashi, and J. Noda, "Rayleigh backscattering measurement of singlemode fibers by low coherence optical time-domain reflectometer with 
$14 \mu \mathrm{m}$ spatial resolution,” Appl.Phys. Lett. 59, 143-145 (1991)

87. F. Feldchtein, V. Gelikonov, R. Iksanov, G. Gelikonov, R. Kuranov, A. Sergeev, N. Gladkova, M. Ourutina, D. Reitze, and J. Warren, "In vivo OCT imaging of hard and soft tissue of the oral cavity," Optics Express 3, 239-250 (1998)

88. B. W. Colston, M. J. Everett, L. B. Da Silva, L. L. Otis, and H. Nathel, "Optical coherence tomography for diagnosing periodontal disease," Proc. SPIE 2973, 216-220 (1997).

89. B. W. Colston; M. J. Everett, L. B. Da Silva, L. L. Otis, P. Stroeve, and H. Nathel, "Imaging of hard- and softtissue structure in the oral cavity by optical coherence tomography," Applied Optics 37, No. 16, 3582-3585 (1998).

90. J. A. Warren, Jr., G. V. Gelikonov, V. M. Gelikonov, F. I. Feldchtein, A. M. Sergeev, N. M. Beach, M. D. Moores, and D. H. Reitze, "Imaging and characterization of dental structure using optical coherence tomography," Optical Society of America Technical Digest Series 6, 128 (1998)

91. W. C. Lee and W. S. Eakle, "Possible role of tensile stress in the etiology of cervical erosive lesions in teeth," J.Prosthetic Dent. 52, 374-380 (1984)

92. Bouma, B. E. , Tearney, G. J., Compton, C. C., Nishioka, N. S., "High-resolution imaging of the human esophagus and stomach in vivo using optical coherence tomography," Gastrointestinal endoscopy, 51(4) Pt 1:467-474 (2000)

93. Aguire, A. D., Herz, P. R., Chen, Y., Fujimoto, J. G., et al, "Ultrahigh resolution OCT imaging with a two-dimensional MEMS scanning endoscope," BIOS 2005, Proc. of SPIE Vol.5692 pp.277

94. Pan, Y., Xie, H., Fedder, G.K. "Endoscopic optical coherence tomography based on microelectromechanical mirror," Optics Letters 26 pp.1966-1968 (2001)

95. Fujino, M., Patterson, P. R. , Nguyen, H., Piyawattanametha, W., Wu, M. C, " Monolithically cascaded micromirror pair driven by angular vertical combs for two-axis scanning," IEEE J. of Selected Topics on Quantum Electronics, Vol.10, No.3, pp. 492-497 (2004) 
96. Patterson, P. R., Hah, D., Nguyen, H., Toshiyoshi, H., Chao, R-m, Wu, M. C. "A scanning micromirror with angular comb drive actuation," MEMS 2002 IEEE Int'1 Conference, 20-24 Jan. 2002

97. Isamoto, K., Makino, T., Kato, K., Morosawa, A., Chong, C., Fujita, H., and Toshiyoshi, H. "A Two-Mask Process for Self-Assembled Vertical Comb Drive Mirrors," IEEE/LEOS Int. Conf. on Optical MEMS \& their applications, Takamatsu, Japan, Aug. 22-26, 2004

98. Glass, A. M.; Schubert, E. F.; Bonner, C. E.; Wilson, B. A.; Cunningham, J. E. "Novel photovoltaic delta-doped GaAs superlattice structure" Applied Physics Letters 54, 2247-2249 (1989)

99. Hirata T, Makita K, Hane K, et al. "Development of MEMS-based optical surge suppressor," OFC 2005, March 6-11, OthD3, Anaheim, California 2005

100. Liu, C. T., Liub, J. M., Choc, A. Y. "Novel photovoltaic transistor directly modulated by steady-state photovoltage in asymmetric superlattices," Electron Devices Meeting, 1991. Technical Digest., International 8-11 Dec. 1991 pp.425 - 428

101. H. Schenk, p Durr, D. Kunze, H. Lakner and H. Kuck, "Design and Modelling of large deflection micromechanical 1D and 2D Scanning Mirrors, " Proceedings of SPIE Vol. 4178 (2000)

102. N. Fujiwara, R. Yoshimura, K. Kato, H. Ishii, F. Kano, Y. Kawaguchi, Y. Kondo, K. Ohbayashi, and H. Oohashi, "140-nm Quasi-Continuous Fast Sweep Using SSG-DBR Lasers," IEEE Photonics Technology Letters, 20, 1015 ( 2008)

103. Connie J. Chang-Hasnain, "MEMS VCSEL," IEEE J. of Selected Topics in Quantum electronics, $\quad 6,978-988$ (2000).

104. Qi Chen, G.D. Cole, E.S. Bjorlin, T. Kimura, Shaomin Wu, C.S. Wang, N.C. MacDonald, J.E. Bowers, "First Demonstration of a MEMS Tunable Vertical-Cavity SOA, " IEEE Photo.Tech.Lett.,16, 1438-1440 (2004)

105. M.Maute, B.Kogel, G.Bohm, P.Meissner, MC. Amann, , "MEMS-Tunable 1.55-_m VCSEL With Extended Tuning Range Incorporating a Buried Tunnel Junction, " IEEE 
Photo.Tech.Lett.18,688-690(2006)

106. O.K.Kwon, K.H Kim, E.D Sim, J.H Kim, and K.ROh, "Proposal of Electrically Tunable External-CavityLaser Diode, " IEEE Photo.Tech.Lett.16, 1804-1806 (2004)

107. O.K.Kwon, K.H Kim, E.D Sim, J.H Kim, and K.ROh, "Monolithically Integrated Multiwavelength Grating Cavity Laser, " IEEE Photo.Tech.Lett., 17, 1788-1790 (2005)

108. L Sun, JH Kim, CH Jang, D An, X Lu, Q Zhou, J.M.Taboada, R.T. Chen, J.J. Mak, S.Tang, H. Zhang, W.H.Steier, C.Zhang, L.R. Dalton, "Polymeric waveguide prism-based electro-optic beam deflector, " Opt. Eng. 40(7) 1217-1222 (2001)

109. A.L. Glebov, M.G. Lee, L.Huang, S. Aoki, Kishio Yokouchi, M. Ishii, and M. Kato, "Electrooptic planar deflector switches with thin-film PLZT active elements," IEEE J. of Selected Topics in Quantum electronics, 11, 422-430 (2005).

110. Y.W. Song, S.A.Havstad, D.Starodubov, Y.Xie, A.E.Willner, J. Feinberg, "40-nm-wide tunable fiber ring laser with single-mode operation using a highly stretchable FBG, " IEEE Photo.Tech.Lett,13, $1167-1169$ (2001)

111. K. Zhang and J.U. Kang, "C-band wavelength-swept ingle-longitudinal mode erbium-doped fiber ring laser," Optics Express 16, 14173 (2008)

112. Mark D. Feuer, "Length and Power Dependence of Self-Adjusting Optical Fiber Filters," IEEE Photo.Tech.Lett. 10, 1587-1589 (1998)

113. S.A. Havstad, B. Fischer, A.E. Willner, M.G. Wickham, "Loop-mirror filters based on saturable-gain or -absorber gratings," Optics Letters 24,1466-1468 (1999)

114. S. Makita, Y. Hong, M. Yamanari, T. Yatagai, and Y. Yasuno, "Optical coherence angiography," Optics Express 14, 7821-7840 (2006)

115. M. Yamanari, S. Makita, V. D. Madjarova, T. Yatagai, and Y. Yasuno, " Fiber-Based Polarization-Sensitive Fourier Domain Optical Coherence Tomography using B-Scan-Oriented Polarization Modulation Method," Opt. Exp. 14,6502-6515 (2006) 
116. J. Zhang, W. G. Jung, J. S. Nelson, and Z. P. Chen, "Full range polarization-sensitive Fourier domain optical coherence tomography, " Opt. Express 12, 6033-6039 (2004)

117. W. Drexler, D. Stamper, C. Jesser, X. Li, C. Pitris, K. Saunders, S. Martin, M. B. Lodge, J. G. Fujimoto, and M. E. Brezinski, "Correlation of collagen organization with polarization sensitive imaging in cartilage: implications for osteoarthritis," J. Rheumatol. 28, 1311-1318 (2001).

118. M. Chilek, S. Raby, and M. E. Brezinski, "Characterizing of tissue microstructure with single-detector polarization sensitive optical coherence tomography," Appl. Opt. 45, 4464-4479 (2006)

119. M.C. Pierce, M. Shishkov, B.H.Park, N.A. Nassif, B.E. Bouma,G. J. Tearney, J. F. de Boer, "Effects of sample arm motion in endoscopic polarization-sensitive optical coherence tomography," Optics Express 13, 573 (2005)

120. G. Zuccaro,N. Gladkova, J. Vargo, F. Feldchtein, E. Zagaynova,D. Conwell, G. Falk, J. Goldblum, J. Dumot, J. Pnsky,G. Gelikonov, B. Davros, E. Donchenko, and J. Richter, "Optical coherence tomography of the esophagus and proximalstomach in health and disease, " Am. J. Gastroenetrol. 96, 2633-2639 (2001).

121. M. E. Brezinski, G. J. Tearney, N. J. Weissman, S. A. Boppart, B. E. Bouma, M. R. Hee, A. E. Weyman,E. A. Swanson, J. F. Southern, and J. G. Fujimoto, "Assessing atherosclerotic plaque morphology: comparison of optical coherence tomography and high frequency intravascular ultrasound," Heart 77, 397-403 (1997).

122. Jason M. Zara, Colleen A. Lingley-Papadopoulos, "Endoscopic OCT Approaches Toward Cancer Diagnosis, " IEEE J. of Selected Topic of Quantum Electronics 14, (2008)

123. M. E. Brezinski, G. J. Tearney, S. A. Boppart, E. A. Swanson, J. F. Southern, and J. G. Fujimoto, "Optical biopsy with optical coherence tomography: feasibility for surgical diagnostics," J. Surg. Res. 71, 32-40 (1997).

124. S. A. Boppart, G. J. Tearney, B. E. Bouma, J. F. Southern, M. E. Brezinski, and J. G. Fujimoto, "Noninvasive assessment of the developing Xenopus cardiovascular system using optical coherence tomography," Proc. Natl. Acad. Sci. 94, 4256-4261 (1997). 
125. Manuel H. De la Torre-Ibarra, Pablo D. Ruiz and Jonathan M. Huntley "Double-shot depth-resolved displacement field measurement using phase-contrast spectral optical coherence tomography," Optics Express 14, 9643 (2006)

126. B. Liu and M. E. Brezinski, "Theoretical and practical consideration on detection performance of time domain, Fourier domain, and swept source optical coherence tomography, " J. Biomedical Optics 12, 044007 (2007)

127. K. Zheng, B. Liu, C. Huang, and M. E. Brezinski, "Experimental confirmation of potential swept source optical coherence tomography performance limitations," Applied. Optics 47,6151-6158(2008)

128. A. M. Sergeev, V. M. Gelikonov, G. V. Gelikonov, F. I. Feldchtein, K. I. Pravdenko, D. V. Shabanov, N. D. Gladkova, V. V. Pochinko, V. A. Zhegalov, G. I. Dmitriev, I. R. Vazina, G. A. Petrova, N. K. Nikulin, "In vivo optical coherence tomography of human skin microstructure," Proc. SPIE 2328, 144-150 (1994).

129. J. M. Schmitt, A. Knuttle, M. J. Yadlowsky, and M. A. Eckhaus, "Optical coherence tomography of dense tissue: statistics of attenuation and backscattering," Phys. Med. Biol. 39, $1705-1720$ (1994).

130. L. Thrane, H. T. Yura, and P. E. Anderson, "Analysis of optical coherence tomography systems based on extended Huygens-Fresnel principle, " J. Opt. Soc. Am. A 17, 484-494 (2000).

131. M. J. Yadlowsky, J. M. Schmitt, and R. F. Bonner, "Multiple scattering in optical coherence microscopy," Applied Optics 34, 5699-5707 (1995).

132. Y. T. Pan, R. Birngruber, and R. Engelhardt, "Contrast limits of coherence-gated imaging in scattering media," Applied Optics 36, 2979-2983 (1997)

133. R. K. Wang, "Signal degradation by multiple scattering in optical coherence tomography of dense tissue: a Monte Carlo study towards optical clearing of biotissues," Phys. Med. Biol. 47, 2281-2299 (2002). 
134. J. M. Schmitt and A. Knuttle, "Model of optical coherence tomography of heterogenous tissue,"J. Opt.Soc. Am. A 14, 1231-1242 (1997).

135. S. G. Adie, T. R. Hillman, and D. D. Sampson, "Detection of multiple scattering in optical coherence tomography using the spatial distribution of Stokes vectors," Optics Express 15, 18033-18049 (2007)

136. Christoph M. Eigenwillig, Benjamin R. Biedermann, Gesa Palte, and Robert Huber, "K-space linear Fourier domain mode locked laser and applications for optical coherence Tomography," Optics Express 16,8916-8937 (2008)

137. Z. Hu, and A. M. Rollins, "Fourier domain optical coherence tomography with a linear-in-wavenumber spectrometer," Optics Letter 32, 3525-3527 (2007)

138. Kathy Zheng, Bin Liu, Chuanyong Huang and Mark E. Brezinski, "Experimental confirmation of potential swept source optical coherence tomography performance limitations," Applied Optics 47, 6151-6158 (2008) 
Publications of work that uses proposed swept source (santec, HSL-1000/2000).

139. "Visualization of Sub-Retinal Pigment Epithelium and Sub-Choroidal Neovascularization Morphologies of Exudative Macular Diseases by Optical Coherence Tomography with Long Wavelength Probe," Yasuno, M.Miura, K.Kawana, S.Makita, et al. Investigative Ophtalmology\&Visual Science

140. "Real-time swept source optical coherence tomography imaging of the human airway using a microelectromechanical system endoscope and digital signal processor,” Jianping Su, Jun Zhang, Lingfeng Yu, Henri G Colt,Matthew Brenner, Zhongping Chena, Journal of Biomedical Optics, Vol. 13 3,030506-1-3 (2008)

141. "In vivo high-contrast imaging of deep posterior eye by $1-\mu \mathrm{m}$ swept source optical coherence tomography and scattering optical coherence angiography,"Yoshiaki Yasuno, Youngjoo Hong1, Shuichi Makita1,Masahiro Yamanari1, Masahiro Akiba, Masahiro Miura and Toyohiko Yatagai, OPTICS EXPRESS 6121 (2007)

142. "In vivo three-dimensional microelectromechanical endoscopic swept source optical coherence tomography," Jianping Su, Jun Zhang, Linfeng Yu, Zhongping Chen, OPTICS EXPRESS 10390 (2007)

143. "Three-dimensional and high-speed swept-source optical coherence tomography for in vivo investigation of human anterior eye segments,"Yoshiaki Yasuno, Violeta Dimitrova, Madjarova and Shuichi Makita,Masahiro Akiba,Atsushi Morosawa, Changho Chong and Toru Sakai,Kin-Pui Chan,Masahide Itoh and Toyohiko Yatagai, OPTICS EXPRESS 10652 $(2005)$

144. "Polarization-sensitive swept-source optical coherence tomography with continuous source polarization modulation," Masahiro Yamanari, Shuichi Makita, Yoshiaki Yasuno, OPTICS EXPRESS 5892 (2008)

145. "High speed frequency swept light source at $1550 \mathrm{~nm}$ for Fourier domain OCT with A-scanning rate at $20 \mathrm{kHz}$," R K Wang, S Cheung, P H Tomlins, C Chong, A Morosawa, D Heard, BIOS2005, 2005.Jan SPIE in SanJose, Californica, USA 
146. "Polarization-sensitive swept-source optical coherence tomography with continuous polarization modulation," Masahiro Yamanari, Shuichi Makita, Toyohiko Yatagai, and Yoshiaki Yasuno Proc. SPIE Vol. 6847, 68471J (2008)

147. "In vivo 3-D imaging of gastrointestinal tracts by use of an endoscopic swept source optical coherence tomography with a microelectromechanical endoscopic rotational probe," Jun Zhang, Jianping Su, Kenneth Chang, and Zhongping Chen, Proc. SPIE Vol. 6847, 68472 Y (2008)

148. "Multi-channel Fourier domain OCT system with superior lateral resolution for biomedical applications," Jon Holmes, Simon Hattersley, Nick Stone, Florian Bazant-Hegemark, and Hugh Barr, Proc. SPIE Vol. 6847, 68470 O (2008)

149. "Full range swept-source optical coherence tomography using $3 \times 3$ Mach-Zehnder interferometer with unbalanced differential detection," Youxin Mao,; Flueraru, Costel; Sherif, Sherif; Shoude Chang, Biomedical Imaging: From Nano to Macro, 2008. ISBI 2008. 5th IEEE International Symposium on Vol, Issue, 14-17 May 2008 Page(s):1355 - 1358"

150. "Forward-Imaging Swept Source Optical Coherence Tomography using Silicon MEMS Scanner for High-Speed 3-D Volumetric Imaging," Kumar, K.; Condit, J.C.; McElroy, A.; Kemp, N.J.; Hoshino, K.; Milner, T.E.; Xiaojing Zhang, Optical MEMS and Nanophotonics, 2007 IEEE/LEOS International Conference on Volume, Issue, Aug. 12 2007-July 162007 Page(s): $10-11$

151. "Delineation of an oral cancer lesion with swept-source optical coherence tomography," Meng-Tsan Tsai, Hsiang-Chieh Lee, Chih-Wei Lu, Yih-Ming Wang, Cheng-Kuang Lee, and C. C. Yang, J. Biomed. Opt., Vol. 13, 044012 (2008); DOI:10.1117/1.2960632, 8 August 2008

152. "Effective indicators for diagnosis of oral cancer using optical coherence tomography," Meng-Tsan Tsai, Hsiang-Chieh Lee, Chih-Wei Lu, Yih-Ming Wang, Cheng-Kuang Lee, and C. C. Yang, Optics Express, Vol. 16, Issue 20, pp. 15847-15862

153. "Development of Phase-Stabilized Swept-Source OCT for the Ultrasensitive Quantification of Microbubbles," R.K.Manapuram, V.G.R.Manne, K.V.Larin, Laser Physics, Vol. 18, No. 9, pp. 1080-1086(2008) 
154. "Endoscopic Optical Coherence Tomography Probe Using Electromagnetically Vibration of Single Fiber, "T.Matsunaga, R.Hino, W.Makishi, M.Esashi, Y.Haga, PROCEEDINGS OF THE 25TH SENSOR SYMPOSIUM,2008.pp.659 662

\section{Reference books}

155. Brett E. Bouma and Guillermo J. Tearney, Handbook of optical coherence tomography (Informa HealthCare, 2001)

156. C. A. Puliafito, M. R. Hee, J. S. Schuman, and J. G. Fujimoto, Optical Coherence Tomography of Ocular Diseases (SLACK, Thorofare, NJ, 1996).

157. Roger Steinert and David Huang, Anterior Segment Optical Coherence Tomography (SLACK, Thorofare, NJ, 2008).

158. Evelyn Regar, A.M.G.J. van Leeuwen, and Patrick W. Serruys, Optical Coherence Tomography in Cardiovascular Research (Informa HealthCare, 2007) 


\section{LIST of PUBLICATIONS}

\section{Journals}

1. Changho Chong, Atsushi Morosawa, Tooru Sakai, "High speed wavelength-swept laser source with High Linearity Sweep for optical coherence tomography," IEEE Journal of Selected Topics of Quantum Electronics, Biophotonics Part1, Volume 14, Issue 1, 235 242, January 1, 2008

2. Changho Chong, Takuya, Suzuki, Atsushi Morosawa, Tooru Sakai, Naoyuki Mekada, "Spectral Narrowing Effect by Quasi-Phase Continuous Tuning in High-Speed Wavelength-Swept Light Source," Optics Express Vol. 16, No. 25, 21105-21118, December 8, 2008, also selected for Virtual J. of Biomedical Optics, V4, Iss.2,21105 Feb.2009

3. Changho Chong, Takuya, Suzuki, Kohki Totsuka, Atsushi Morosawa, Tooru Sakai, "Large Coherence Length Swept Source for Axial Length Measurement of Eye ",Applied Optics(Topics; Biomedical Optics) to be published in 2009 Apr.

4. Changho Chong, Isamoto Keiji, Jonathan Evans, Hiroshi Toshiyoshi, "Optically Modulated MEMS Scanning Endoscope," IEEE Photonics Technology Letters, Vol. 18, No. 1, 133-135, January 1, 2006

5. Changho Chong, Takuya, Suzuki, Kohki Totsuka, Atsushi Morosawa, Tooru Sakai, "High-speed wavelength swept laser source with double deflection geometry for optical coherence tomography," Optics Letter, Submitted in Dec. 2008

6. Changho Chong, T.Suzuki, K.Totsuka, A.Morosawa, T.Sakai, "High-speed swept source optical coherence tomography with using Spectrum Sliced Swept Source," To be submitted 
7. C.Chong, T.Suzuki, K.Totsuka, A.Morosawa, T.Sakai, "High deflection angle optically modulated MEMS scanning fiber probe for Optical Coherence Tomography," To be submitted

\section{International Conferences}

8. Changho Chong, Kohki Totsuka, Takuya Suzuki, Atsushi Morosawa, Tooru Sakai," Intraocular distance measurement with using high coherence swept-source OCT," The 4the Asian and Pacific Rim Symposium on Biophotonics, Jeju, Korea, $27^{\text {th }}$ May, 2009 (Scheduled)

9. Changho Chong, Takuya, Suzuki, Atsushi Morosawa, Tooru Sakai, Naoyuki Mekada, "Coherence Length Improvement by Spectral Narrowed Wavelength-Swept Laser with Quasi-Phase Continuous Tuning," Biomedical Optics topical mt OSA 06, St.Petersberg, Florida, 2008.3.21

10. Changho Chong, Atsushi Morosawa, Tooru Sakai, "High speed wavelength-swept laser source with simple configuration for optical coherence tomography," SPIE European Conference on Biomedical Optics 2007.6.17, Munich, Germany

11. Changho Chong, Isamoto Keiji, Jonathan Evans, H. Toshiyoshi, "Optically-modulated MEMS Scanning Endoscope for Optical Coherence Tomography," Biomedical Optics topical mt OSA 06, Fortloderdale, Florida, 2006.3.21

12. Changho Chong, Isamoto Keiji, Jonathan Evans, Yasuki Sakurai, Nakada Muneki, Hiroshi Toshiyoshi, Hiroyuki Fujita, "Optically Actuated MEMS Scanning Fiber Probe for Medical Applications," World Congress in Medical Physics and Biomedical Engineering, 2006.8.30, Seoul, Korea

13. C.Chong, K. Isamoto, M. Nakada, H. Fujita, and H.Toshiyoshi, "A Photovoltaically Modulated MEMS Optical Scanner for Fiber Endoscope," Int. Conf. on Microtechnologies in Medicine and Biology (MMB 2006), May 9-12, 2006, Bankoku-Shinryokan, Okinawa, Japan.

14. Changho Chong, Keiji Isamoto, Atsushi Morosawa, Kazuya Kato, Hiroyuki Fujita, and Hiroshi Toshiyoshi, "MEMS variable optical attenuator with simple and smart design for stable and reliable operation," Proc. SPIE vol. 5279, pp. 40-48, 2004.

15. C. Chong, K. Isamoto, H. Fujita, and H. Toshiyoshi, "MEMS variable optical attenuator with simple and smart designfor stable and reliable operation," Asia-Pacific Optical and 
Wireless Communications (APOC 2003), Nov. 2 - 6, 2003, Wuhan Science and Technology Conference \& Exhibition Center, Wuhan, China, (Invited)

16. C. Chong, K. Isamoto, H. Fujita, and H. Toshiyoshi, "Variable Optical Attenuator with Simple SOI-MEMS Mirror," Proc. the 29th European Conference on Optical Commulation / the 14th Int. Conf. on Integrated Optics and Optical Fiber Communication (ECOC / IOOC 2003), Sep. 21-25, 2003, Rimini, Italy, Mo-3.5.2

17. Atsushi Morosawa, Changho Chong, Tooru Sakai, "Wide tuning range wavelength-swept laser with single semiconductor optical amplifier for OCT," SPIE European Conference on Biomedical Optics 2007.6.17 Munich

18. Yoshiaki Yasunoaki, Violeta Dimitrova Madjarovaa, Shuichi Makita, Masahiro Akibab, Atsushi Morosawa, Changho Chong, Toru Sakaic, Kin-Pui Chanb, Masahide Itoha and Toyohiko Yatagaia, "Three dimensional investigation of in vivo anterior eye segments by swept-source optical coherence tomography with ready-for-shipping scanning light source," BIOS2005, 2005.Jan SPIE in San Jose, Californica, USA

19. R K Wang, S Cheung, P H Tomlins, C Chong, A Morosawa3 D Heard, "High speed frequency swept light source at $1550 \mathrm{~nm}$ for Fourier domain OCT with A-scanning rate at 20kHz," BIOS2005, 2005.Jan SPIE in San Jose, Californica, USA

20. Muneki Nakada, Changho Chong, Keiji Isamoto, Hiroyuki Fujita, and Hiroshi Toshiyosh, "Micro Optomechanical Devices for Medical Endoscope Applications," SPIE Optics East ---Photonics for Applications in Industry, Life Sciences, and Communication---, Oct. 1-4, 2006, Hynes Convention Center, Boston, MA, USA, Optomechatronics Technologies Conference Proc. SPIE Vol. 6376 (invited).

21. M. Nakada, H. N. Kwon, C. Chong, A. Morosawa, K. Isamoto, H. Fujita, and H. Toshiyoshi, "Optical coherence tomography based on power-over-fiber MEMS scanner," in Proc. 4th Asia Pacific Conference on Transducers and Micro-Nano Technology (APCOT 2008), June 22-25, 2008, TAYIH Landis Hotel, Tainan, Taiwan R.O.C., p. 2A2-3.

22. M. Nakada, C. Chong, K. Isamoto, A. Morosawa, H. Fujita and H. Toshiyoshi, "Design and Fabrication of Silicon Bulk Micromachined Optical Scanner for Medical Endoscope," in Proc. 14th Int. Display Workshop, Sapporo Convention Center, Sapporo, Japan, Dec. 5-7, 2007 
23. M. Nakada, H. Fujita, H. Toshiyoshi, C. Chong, K. Isamoto, "Wavelength Division Multiplexing for MEMS-based Fiber Optic Endoscope," Proc. 5th Int. COnf on Optics-Photonics Design \& Fabrication (ODF '06), Dec. 6-8, 2006, Nara-Ken New Public Hall, Nara, Japan (invited).

24. M. Nakada, C. Chong, K. Isamoto, H. Fujita, and H. Toshiyoshi, "Design and fabrication of optical MEMS scanners for optically modulated fiber endoscopes," Proc. IEEE/LEOS Int. Conf. on Optical MEMS and Their Applications (Optical MEMS 2006), Big Sky Resort, Big Sky, Montana, Aug. 21-24, 2006

25. M. Yoda, K. Isamoto, Changho Chong, H. Ito, S. Kamisuki, M. Atobe, and H. Toshiyoshi, "A MEMS 1-D Optical Scanner for Laser Projection Display using Self-assembled Vertical Combs and Scan-angle Magnifying Mechanism," Proc. 13th Int. Conf. on Solid-State Sensors, Actuators and Microsystems (TRANSDUCERS '05), June 5 - 9, 2005, Seoul, Korea

26. Keiji Isamoto, Tatsuya Makino, Kazuya Kato, Atsushi Morosawa, Changho Chong, Hiroyuki Fujita, and Hiroshi Toshiyoshi, "A Two-mask Process for Self-assembled Vertical Comb Drive Mirrors," IEEE/LEOS Int. Conf. on Optical MEMS and Their Applications (Optical MEMS 2004), Takamatsu, Japan, Aug. 22-26, 2004, pp.172-173

27. Keiji Isamoto, Kazuya Kato, Atsushi Morosawa, Changho Chong, Hiroyuki Fujita, and Hiroshi Toshiyoshi, "Micromechanical VOA Design for High Shock-Tolerance and Low Temperature-Dependence," IEEE/LEOS Int. Conf. on Optical MEMS and Their Applications (MOEMS 03), Outrigger Waikoloa Beach, Hawaii, USA, Aug. 18 - 21 , 2003.

28. H. Toshiyoshi, K. Isamoto, A. Morosawa, M. Tei, and H. Fujita, "A 5-Volt Operated MEMS Variable Optical Attenuator," Proc. the 12th Int. Conf. on Solid-State Sensors, Actuators and Microsystems (Transducers 03), Boston Marriott Copley Place, Boston, MA, USA, June 8-12, 2003,4D1.2, pp. 1768-1771

29. R.Nakamura, A.Murata, C.Chong, K.Yano, S.Kamisuki, "Highly wavelength-selective MEMS Fabry-Perot Tunable Filter," 22nd Sensor Symposium on Sensors, Micromachine and Applied Systems, oct20-21,2005, Tower Hall Funabori, Tokyo 


\section{Relevant Journals}

30. Yoshiaki Yasuno, Violeta Dimitrova, Madjarova and Shuichi Makita, Masahiro Akiba, Atsushi Morosawa, Changho Chong and Toru Sakai, Kin-Pui Chan, Masahide Itoh and Toyohiko Yatagai , "Three-dimensional and high-speedswept-source optical coherencetomography for in vivo investigation ofhuman anterior eye segments, "26 December 2005 / Vol. 13, No. 26 / OPTICS EXPRESS 10652

31. Nobuyoshi Ozawa, Yasunori Sumi, Changho Chong, Isao Nishida, "First in vivo OCT Images of Human Labial Glands," Radiology (Submitted in September, 2008)

32. N. Ozawa, Y.Sumi, C. Chong, T.Kurabayashi, "Evaluation of oral vascular anomalies using OCT imaging," (Submitted)

33. Keiji Isamoto, Kazuya Kato, Atsushi Morosawa, Changho Chong, Hiroyuki Fujita, Hiroshi Toshiyoshi, "A 5-V Operated MEMS Variable Optical Attenuator by SOI Bulk Micromachining," IEEE JOURNAL OF SELECTED TOPICS IN QUANTUM ELECTRONICS, VOL. 10, NO. 3, MAY/JUNE 2004

\section{Domestic Conferences}

34. “波長走査型光源の単一偏光発振, " 酒井徹, 鄭 昌鎬, 鈴木卓也, 三国之也, 両澤 淳,女鹿田, 応用物理学会 2008 年秋 (中部大学) 3.7 生体医用光学

35. "光周波数掃引型 OCT 用光源の特性とOCT システム性能との相関," 両澤 淳, 鄭 昌鎬, 酒井徹 OpticsJapan, 第4 回生体医用光学研究会, 2005.11.24 日本光学 会

36. "高速Swept Source OCT によるinvivo 3 次元トモグラフィー, "安野嘉晃, 巻田修 一,V.D. Madjarova，秋葉正博，両澤淳，鄭昌鎬，酒井徹，陳建培，伊藤雅英，谷田貝 豊彦，応用物理学会 2005 年秋(徳島大学) 3.7 生体医用光

37. "光ファイバ内視鏡用光駆動MEMS スキャナシステム," 中田宗樹（東京大学）、鄭 昌鎬, 諫本圭史（サンテック）, 藤田博之, 年吉 洋（東京大学）マイクロマシン・ センサシステム研究会,平成 18 年 7 月 27 日（木）,立命館大

\section{Other publications}


38. 角保徳，西田功，鄭昌鎬，梅村長生，“光干渉断層画像診断法の歯科臨床への応用,” 日本歯科医師会雑誌 第60巻 12 号、（2008、3月号）, 医師会誌

39. "High Speed Wavelength Scanning Laser Creates New Applications," C.Chong, D.Heard, Europhotonics, December issue. 2005, 雑誌,

40.「光デバイス精密加エハンドブック」MEMS光アッテネータ寄稿

41. Contribution of Chapter 9. Reliablity of MEMS variable optical attenuator, H.Toshiyoshi, K.Isamoto, C.Chong, "Reliability of MEMS: Testing of Materials and Devices (Advanced Micro and Nanosystems), Wiley-VCH (February 15, 2008)

\section{Symposiums}

42.「高速/広带域/連続波長走査型レーザの開発」, 2005-2006年度 光産業技術振興 協会＼cjkstart開発プロジェクト報告書（2006FY-010-1）

43.「OCT用高性能波長走查光源」21世紀のバイオメディカルフォトニクス, 2006.12.1,

44.「光コヒーレンストモグラフィ用光源技術」,フォトニクスイノベーションシンポ ジウム 2007.11.30, 京都大学

\section{Patents}

Domestics patent applications

1. 特開2008-305997 波長走査型レーザ光源

2. 特開2008-277683 波長走査型レーザ光源

3. 特開2008-261778 光断層画像表示システム及び光断層画像表示方法

4. 特開2008-175867 波長可変フィルタ

5. 特開2008-157759 光ファイバセンサシステム

6. 特開2008-098395 波長走查型レーザ光源

7. 特開2008-058138 歯科用光断層画像表示システム

8. 特開2007-278868 光干渉断層画像表示システム

9. 特開2007-234786 波長可変レーザ光源

10. 特開2007-229310 歯科用光断層画像表示システム

11. 特開2007-042971 波長走查型レーザ光源 
12. 特開2007-027306 波長可変レーザ光源

13. 特開2007-024677 光断層画像表示システム

14. 特開2006-293054 光偏向プローブ及び光偏向プローブ装置

15. 特開2006-237359 波長走査型ファイバレーザ光源

16. 特開2006-235606 波長可変フィルタおよび波長可変フィルタの製造方法

17. 特開2006-208791 波長可変フィルタおよび波長可変フィルタの製造方法

18. 特開2006-080384 波長走査型ファイバレーザ光源

19. 特開2006-060966 M E M S 素子及びデバイス

20. 特開2006-047897 M E M S 素子、その製造方法及び光デイバイス

21. 特開2006-024876 波長走査型ファイバレーザ光源

22. 特開2005-347668 波長走査型ファイバレーザ光源

US patents

1. 7,489,713 Tunable fiber laser light source

2. 7,382,809 Tunable fiber laser light source

3. 7,352,783 Tunable fiber laser light source

4. 7,323,680 Optical deflection probe and optical deflection probe device

5. 7,099,358 Tunable laser light source

US patent applications

1. 20080252899 Optical coherence tomography system and optical coherence tomography method

2. 20080159468 Optical coherent tomography 


\section{List of Figures}

1.1 Absorption and scattering coefficients of light 4

1.2 Requirements of swept source for different clinical application 6

1.3 Spectral dynamics of a swept-source 9

1.4 Fourier Domain Mode Locked laser source(top), ECL with polygon scanner-based filter $\begin{array}{ll}\text { with Telescopic lens configuration } & 11\end{array}$

$\begin{array}{lr}1.5 \text { Organization of thesis } & 15\end{array}$

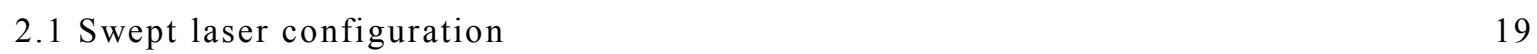

2.2 Temporal evolution of beam deflection inside the filter block 19

2.3 Linearity over frequency range with different $\beta \quad 23$

2.4 Linearity over frequency range with different $\Lambda \quad 23$

$\begin{array}{ll}2.5 \text { Filter bandwidth over wavelength range } & 24\end{array}$

2.6 Simulated point spread functions (signal spectrum) 25

2.7 Depth dependence of axial resolution 25

$\begin{array}{ll}2.8 \text { Scan rate and scan range vs. the number of polygon facet } & 27\end{array}$

2.9 Output spectrum of the laser source per scan at $20 \mathrm{kHz}$ scan rate 29

2.10 Photograph of the scanning filter block and 3D CAD schematic of fabricated laser $\begin{array}{ll}\text { source } & 30\end{array}$

2.11 Measured linearity of the scan in dots and theoretical line 31

2.12 Relative intensity noise at $20 \mathrm{kHz}$ scanning rate 31

2.13 SS-OCT system configuration $\quad 32$

$\begin{array}{ll}2.14 \text { Power spectrums } & 34\end{array}$

2.15 Power spectrums at $1 \mathrm{~mm} \quad 35$

2.16 Axial resolutions as a function of image depth 35

2.17 OCT image of human finger measured with a swept source with (a)(c) $0.8 \%$ linearity, (b) $0.25 \%$ linearity sweep $\quad 37$

3.1 Diagram of multi-mode lasing with Gaussian filter envelope 40

3.2 (a) Configuration for phase continuous tuning for mode-hop free tuning (b) Concept of quasi-phase continuous tuning $\quad 41$

3.3(a) Phase diagram of Lasing in Fig 2(a) (b) Phase diagram of Lasing in Fig 2(b) 41

3.4 QPCT conditions with different wavelength ranges 44

3.5 Phase variation over diffraction angle range, Cavity mode order over wavelength 44 
3.6 Out-of-Phase ratio P over wavelength range 44

3.7 Diagram of polygon scanner -based swept laser source $\quad 47$

3.8. Temporal optical output power $\quad 48$

3.9. Relative Intensity Noise 48

3.10. Balanced Mach-Zehnder interferometer 48

3.11.(a) OCT signal at different depth positions with and without QPCT condition 49

(b) Close-up of spectrum (20 kHz swept rate)

3.12. OCT signal at different depth positions with and without QPCT condition $\quad 49$

3.13. Spectral linewidth vs. Scan rate 50

3.14. OCT signal at different depth positions with and without QPCT condition $\quad 50$

3.15 Schematic cross-section of a healthy human eye $\quad 52$

3.16 Laser configuration $\quad 54$

3.17 Phase change over the range $\quad 54$

3.18 Phase shift ratio over wavelegth range $\quad 54$

3.19 Filter bandwidth vs. FSR of tunable filter 56

3.20 Temporal profile of output power 56

3.21 OCT system setup for IOL measurement $\quad 57$

$\begin{array}{ll}3.22 \text { Signal power at different depth } & 58\end{array}$

3.23 IOL measurement of the pig-eye $\quad 59$

$\begin{array}{ll}3.24 \mathrm{IOL} \text { measurement of the human eye } & 60\end{array}$

$\begin{array}{ll}3.25 \text { Light vs. carrier density for n-th cavity mode } & 63\end{array}$

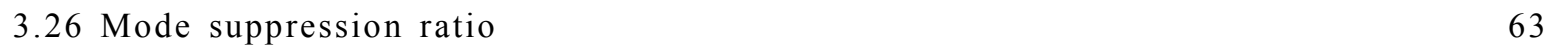

3.27 Output power vs. Injection current with additional cavity loss 63

$\begin{array}{ll}3.28 \text { Spectrum comparison } & 63\end{array}$

$\begin{array}{ll}4.1 \text { Output spectrum of the SOA } & 68\end{array}$

$\begin{array}{ll}4.2 \text { Comparison of Littman configuration } & 70\end{array}$

$\begin{array}{ll}4.3 \text { Beam walk-off issue } & 70\end{array}$

4.4 Spatial Spectral Shaping Technique $\quad 71$

$\begin{array}{ll}4.5 \text { Output spectrum of the laser } & 72\end{array}$

4.6 Temporal intensity and wavelength profile $\quad 72$

$\begin{array}{ll}\text { 4.7 Peak-hold mode spectrum with OSA } & 73\end{array}$

4.8 Swept source in a double pass Littrow configuration $\quad 74$

4.9 (a) Filter bandwidth, (b) Amplitude of interference fringes vs. depth $\quad 74$

4.10 Mechanism of single polarization recovery module $\quad 75$

4.11 Synchronous double deflection $\quad 77$

4.12(a) FSR vs Scan rate (b)Scan rage, Coherence length vs Scan rate 79

4.13 Laser configuration $\quad 80$

4.14 (a)Output power spectrum $\quad$ (b)OCT signal power at different depths $\quad 80$ 
$\begin{array}{ll}5.1 \text { OCT interferometer } & 84\end{array}$

$\begin{array}{ll}\text { 5.2.SS-OCT system } & 88\end{array}$

5.3. 3D OCT image of human skin(finger) $\quad 88$

$\begin{array}{ll}5.4 \text { Hardware configuration } & 89\end{array}$

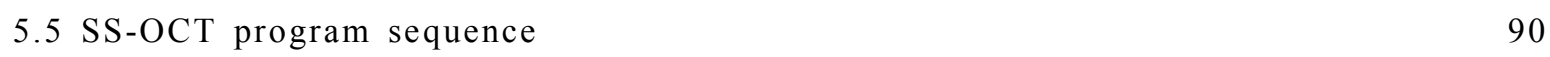

5.6 Example of SS-OCT system display 91

5.7 Photograph of a developed handheld probe $\quad 91$

5.8 Comparison of X-ray and OCT image of the tooth 92

5.9 3D image of tooth with emphasized alveolar bone 92

5.10. Resin filling 93

$5.113 \mathrm{D}$ OCT image of caries 93

5.12 OCT image of a capillary-venous malformation of the buccal mucosa 94

5.13 3D OCT image of human Labial glands 94

5.14 Diagram of Anterior chamber of human eye $\quad 95$

5.15 SS-OCT image of Anterior chamber of in-vivo human eye 95

$5.163 \mathrm{D}$ OCT image of anterior segment 96

5.17. Rotary type scanning catheter for OCT (ref [40]) 97

5.18 Schematic diagram of optically modulated MEMS probe, OCT system configuration 98

5.19 MEMS tilt mirror w/ angular vertical comb drive 102

5.20. MEMS fabrication process step with bulk SOI wafer 102

$\begin{array}{ll}5.21 \text { Mechanism of buckling of stiction pad } & 103\end{array}$

5.22. (a) Schematic diagram of photovoltaic cell and (b)generated voltage characteristics 104

5.23 Response of photovoltaic cell to the optical intensity modulation 105

$\begin{array}{lr}5.24 \text { Bandpass filter characteristics } & 106\end{array}$

5. 25 MEMS mirror characteristics 107

$\begin{array}{lr}5.26 \text { Experimental setup for validating the optical actuation } & 108\end{array}$

$\begin{array}{lr}5.27 \text { Photograph of fabricated MEMS fiber probe } & 108\end{array}$

5.28 SS-OCT image taken with handheld probe with Galvano mirror (a) with the fabricated

$\begin{array}{lr}\text { MEMS mirror (b). } & 109\end{array}$

5.29 Photograph of MEMS mirror $\quad 110$

5.30 Photograph of Photovoltaic cell and its voltage vs optical power 111

5.31. Diagram of improved MEMS probe design and photograph the fabricated sample 112

5.32 Experimental setup for validation of optical modulation 113

5.33 Future design consideration of compact MEMS probe 113 
6.1 Conventional Tunable VCSEL[13] 116

6.2 Proposed MEMS hybrid tunable VCSEL 116

$\begin{array}{ll}6.3 \text { Relation of applied voltage } & 117\end{array}$

6.4 Relation of FSR and Air-gap 117

$\begin{array}{ll}6.5 \text { Proposal of EO deflection for tenability } & 118\end{array}$

6.6 Monolithic EO tunable ECL [107] 118

6.7 Polymer EO deflector with cascaded electrode section $\quad 118$

6.8 (a) Pivot tuning (b) Non-pivot tuning as in QPCT 119

6.9 Proposed Hybrid EO deflector ECL type swept source 119

$\begin{array}{lr}6.10 \text { Definitions of geometrical parameters } & 120\end{array}$

6.11 (a)Phase change vs. diffraction angle (b) Mode hop number $\quad 120$

6.12 (a)Phase change vs. diffraction angle (b)Mode hop number $\quad 120$

6.13 Single mode tunable fiber laser w/ self induced saturable absorber filter 121

6.14 Proposed configuration with saturable absorber filter $\quad 121$

A.1 OCT B-scan of in vivo human macula captured using (left) $1-\mu \mathrm{m}$ OCT and (right)

830-nm SD-OCT. (taken from ref [39]) 126

A.2 3D volume-rendered OCT images of in vivo human macula (taken from ref [39]) 127

A3. Intensity (upper left), phase retardation (upper right) and orientation (lower right) 127

A4 (a) Three-dimensional rebuilt image of rabbit trachea 128

A5. SS-OCT scanning images of the cancerous (a) and normal oral mucosal (b) tissues 128

A6. OCT image of Tadpole (Xenopus laevis, Albino, 2weeks, 2mm in size of head ) 129

A7 OCT image of Embryo of Tadpole after 24 hrs from first cell division 129

$\begin{array}{lr}\text { A8. OCT image of leaf } & 130\end{array}$

$\begin{array}{lr}\text { A9. Orange skin } & 130\end{array}$

A10.Three dimensional dtructure of MEMS pressure sensor $\quad 131$

A10 Plastic molding 132

A12.OCT image of disposable contact lens floating inside the container case 132

A13 Paint on the metal 132

A14. SS-OCT system from MDL 134

A15. SS-OCT image of human skin with $1 \mathrm{~mm}$ source(top)and $1.3 \mu \mathrm{m}$ source(bottom) 134

A16. SS-OCT system for anterior chamber diagnostics (SS-1000) 135 


\section{List of Tables}

1.1 Comparison of different tunable laser technologies $\quad 8$

$\begin{array}{lr}1.2 \text { List of references of swept sources } & 12\end{array}$

$\begin{array}{lr}2.1 \text { Reliability test results } & 28\end{array}$

3.1. List of summary results 50

4.1 Typical specification of SOA (COVEGA: BOA1132) 65

5.1 MEMS target specifications 96

5.2 MEMS mirror (MSM1D) specification 106

A1. Reference of the work that uses our swept-source in SS-OCT 132 